\title{
Alkali-Metal-Mediated Synergistic Effects in Polar Main Group Organometallic
}

\section{Chemistry}

Stuart D. Robertson, ${ }^{a}$ Marina Uzelac, ${ }^{\text {ab }}$ and Robert E. Mulvey ${ }^{a *}$

a WestCHEM, Department of Pure and Applied Chemistry, University of Strathclyde, 295 Cathedral Street, Glasgow, G1 1XL, UK

${ }^{b}$ Current address: School of Chemistry, The University of Manchester, Oxford Road, Manchester, M13 9PL, UK

ABSTRACT: The development of synthetic chemistry since the early 1900 s owes much to the service of organolithium reagents. Brilliant bases (e.g., deprotonating $\mathrm{C}-\mathrm{H}$ bonds), nucleophiles (e.g., adding to unsaturated molecules) and transfer agents (e.g., delivering ligands to other metals), these versatile virtuosi and to a lesser extent the organic derivatives of the other common alkali metals sodium and potassium have proved indispensable in both academia and technology. Today these monometallic compounds are still utilized widely in synthetic campaigns but in recent years they have been joined by an assortment of bimetallic formulations that also contain an alkali metal but in company with another metal. These bimetallic formulations often exhibit unique chemistry that can be interpreted in terms of synergistic effects, for which the alkali metal is essential though it is often the second metal that performs the synthetic transformation. Here, this "alkali-metal-mediated" chemistry is surveyed focusing mainly on bimetallic formulations containing two alkali metals or an alkali metal paired with magnesium, calcium, zinc, aluminum or gallium. In this International Year of the Periodic Table (IYPT) we ponder whether a Pairiodic Table of Element Pairs will emerge in the future. 


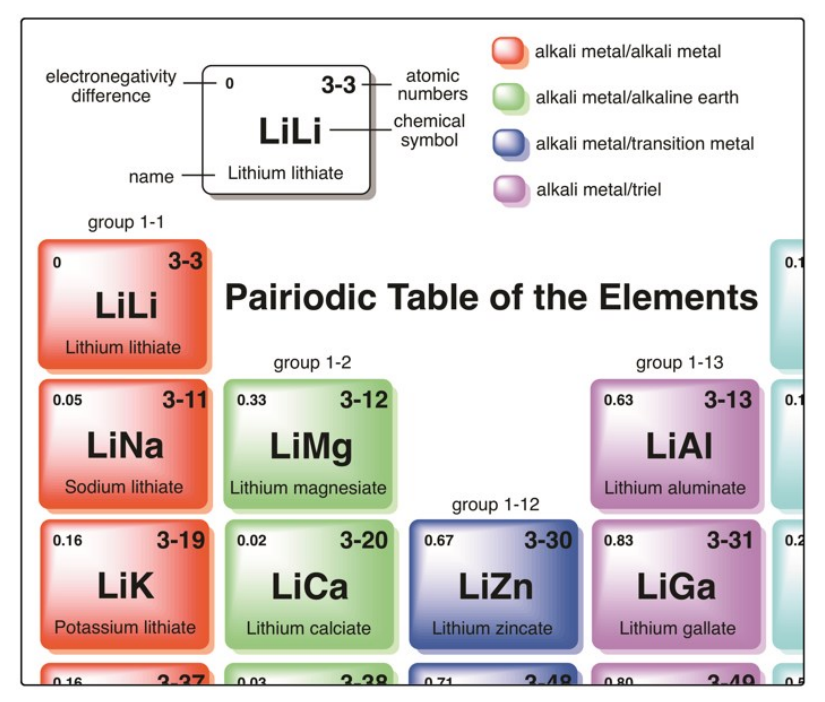

\section{CONTENTS}

1. Introduction

2. Heterometallic Synergistic Main Group Complexes

2.1. Mixed Alkali-Metal Complex Chemistry

2.1.1. The Lochmann-Schlosser Superbase and Related Systems

2.1.2. Other Alkali Metal - Alkali Metal Systems

2.2 Group 2 Chemistry

2.2.1 Inverse Crown and Related Complexes

2.2.2. Magnesiates Involving Trimethylsilylmethyl and Related Ligands

2.2.3. Magnesium and Calcium Chemistry Involving Dianionic Butadienyl Ligands

2.2.4. Heterobimetallic Hydrides

2.2.5. Magnesiate and Related Complexes Synthesized via Di-nbutylmagnesium

2.2.6. Applications in Homogeneous Catalysis and Polymerization Chemistry

2.2.7. Special Metathesis Reactions 
2.2.8. Miscellaneous Complexes

2.3. Turbo-Grignard Reagent and Related Salt-Supported Complex Chemistry

2.3.1. Introduction

2.3.2. Turbo Reagents in Metal-Hydrogen Exchange: Background

2.3.3. Turbo Metal-Hydrogen Exchange Reactions: Recent Advances

2.3.4. Solid Air- and Moisture-Resistant Turbo-Zinc Pivalate-Containing

Reagents

2.3.5. Turbo Reagents in Metal-Halogen Exchange Reactions

2.3.6. Reactions Performed Under Continuous Flow Conditions

2.3.7. Reactions Involving Direct Metal Insertion

2.3.8. Transition-Metal-Free Cross-Coupling Applications

2.4. Group 12 Chemistry

2.4.1. TMP-zincate and Related Metalating Complexes

2.4.2. Uses in $\mathrm{N}$-Heterocyclic Carbene (NHC) Chemistry

2.4.3. Special Cocomplexation Chemistry of Zincates

2.4.4. Trans-Metal-Trapping

2.4.5. Miscellaneous Examples

2.5. Group 13 Chemistry

2.5.1. Lithium Aluminum Hydride - The Original Alkali-Metal Aluminate

2.5.2. Modified Forms of $\mathrm{LiAlH}_{4}$

2.5.3. Trans-Metal-Trapping

2.5.4. Miscellaneous Examples

2.5.5. Looking to the Future

3. Conclusion and Perspectives 
Author Information

Corresponding Author

ORCID

Notes

Biographies

Acknowledgements

Abbreviations

References

\section{INTRODUCTION}

The timeline of synergistic bimetallic chemistry goes back essentially to the beginning of organometallic chemistry when Wanklyn announced the first alkali metal zincate $\left[\mathrm{Na}\left(\mathrm{ZnEt}_{3}\right)\right] \cdot \cdot^{1,2}$ The train of thought behind this is that metalate (ate) complexes (for example, magnesiates, zincates, aluminates etc.) by their very definition must be synergistic compounds in possessing two (or more) distinct metals that in contacted ion pair structures would both participate in the transition states of reaction intermediates or in charge-separated structures would induce the charge separation by the monovalent alkali metal transferring its valence electron to the more electronegative softer metal (for example, magnesium, zinc or aluminum). In practice, the neutral magnesium, zinc or aluminum moieties of these compounds, when separated from the alkali metal moieties, generally cannot reproduce the chemistry of the bimetallic compounds. This is particularly manifest in metalation (C-H to Cmetal exchange) chemistry, since these individual metals make weak neutral 
organometallic bases that are generally incapable of deprotonating the $\mathrm{C}-\mathrm{H}$ bonds of aromatic molecules, let alone the more challenging $\mathrm{C}-\mathrm{H}$ bonds of aliphatic compounds.

How extensive is ate chemistry? There is no easy answer to this question. Theoretically, every neutral organometallic compound in the periodic table can be transformed into ate modifications of every other metal in the periodic table (e.g., lithium magnesiates, lithium aluminates, lithium zincates and so on), even in the same group (e.g., sodium lithiates) or with itself (lithium lithiates). Factor in valency (e.g., divalent $\mathrm{Mg}$, trivalent $\mathrm{Al}$ ), multiple ligand permutations as in heteroleptic systems, stoichiometric variance (e.g., $R_{3} L i Z n, R_{4} L i{ }_{2} Z n, R_{5} L i Z n_{2}$ ), structural distinctions (e.g., charge-separated ion pairs, contacted ion pairs) and solvent-free and solvated derivatives, then one arrives quickly at the realization that the number of possible ate compounds is inestimable. Moreover, the classification 'ate' does not fit every bimetallic compound, for example in cases where two different monovalent metals are simultaneously attached to the same dianionic ligand.

When it comes to bimetallic systems that exhibit synergistic effects the picture gets rather blurred. Few authors explicitly interpret their chemistry in terms of synergistic effects. Those that do, in the clearest cut examples, draw compelling comparisons between the behavior of the bimetallic compound and those of the parent monometallic compounds from which the bimetallic compound is constructed. The alkali metals being the most electropositive elements in the periodic table induce strong polarization effects in bimetallic compounds, the degree of which is dependent on the alkali metal, hence synergistic effects tend to be more prominent in bimetallic 
compounds, especially ates, containing an alkali metal. This strong polarization is one important factor in promoting synergistic behavior, especially when the companion metal is complementary to the "hard" alkali metals in being a "softer" metal in the HSAB (hard-soft acid-base) sense. Since structure and reactivity are inextricably linked, the pre-eminent factor behind synergistic conduct is the structure of the bimetallic reagent, which by definition must be different from that of its parent single metallic components. In general, the bimetallic structure will contain multiple Lewis acidic and Lewis basic sites thus increasing opportunities for building transition state geometries with substrates and hence for reacting with organic substrates in special ways inaccessible to conventional monometallic compounds.

For this review it was decided to focus on bimetallic compounds containing at least one alkali metal for which there was tangible evidence for their structure and that in most cases exhibited (as either expressed by the authors or in our own judgement) some degree of synergic reactivity or have the potential to do so but have not yet been studied in depth. The scope of the article is therefore limited mainly to molecular systems utilized in non-aqueous solvents in organic applications, with solidstate chemistry and aqueous-based chemistry excluded. Currently the most welldeveloped cases involve magnesium, zinc, or aluminum as the companion metal, hence the review has been arranged along periodic lines covering Group 1, Group 2, Group 12, and Group 13. One class of reagent that stands head and shoulders above all others are the so-called "turbo-reagents". Bona fide synergistic reagents, their utility is so vast, greater than all others combined, they are deserving of a chapter in their own right. 
Two huge reviews appeared in this journal in 2013 comprehensively covering the structures and syntheses/reactivities of dipolar organometallic aggregates respectively. ${ }^{3,4}$ These reviews have included all bimetallic compounds exhibiting synergistic effects albeit in a good proportion of cases their chemistry has not been placed explicitly in a synergistic context. To avoid unnecessary repetition, this present review will therefore cover mostly examples since that time, and as you can ascertain from the size of this review, progress within this synergistic theme has been substantial, though earlier literature will be dipped in and out of for aiding understanding and for placing work in its proper context. Note that since this review is written from an inorganic-organometallic-structural perspective, one should refer to the original literature for full details of the vast array of electrophilic trapping and cross-coupling methods used for onward organic transformations, as only general patterns will be discussed here. All molecular structures have been plotted using thermal ellipsoids from data obtained via the Cambridge Crystallographic Database. Hydrogen atoms and disorder have been omitted with the exception of hydrides which require to be shown as they are a key feature of the overall structure.

\section{HETEROMETALLIC SYNERGISTIC MAIN GROUP COMPLEXES}

\subsection{Mixed Alkali-Metal Complex Chemistry}

\subsubsection{The Lochmann-Schlosser Superbase and Related Systems}

If asked for an example of a synergistic reagent, chemists with knowledge of polar organometallic chemistry would most probably answer the Lochmann-Schlosser superbase. ${ }^{5,6}$ This binary mixture of $n$-butyllithium and potassium $t$-butoxide is in fact 
the epitome of a synergistic reagent. The development of this superbase was predated by the work of Morton, who pioneered the practice of mixing metal alkyl compounds with alkali metal alkoxides. Researching polymerisation processes, Morton clearly recognised the synergistic advantages of such combinations, through quotes such as "as a working hypothesis, the accelerating influence that a non-metallating agent, the alkoxide, has on the metallating ability of a very reactive organoalkali metal reagent can be explained by the assumption that a complex anion $\left[\mathrm{C}_{5} \mathrm{H}_{11} \mathrm{Na}(\mathrm{OR})\right]^{-} \mathrm{Na}^{+}$, is present" ${ }^{7}$ Both useful reagents in their own right, when $n$-butyllithium and potassium $t$-butoxide are combined they can perform chemistry outside the scope of the separated entities (Scheme 1). ${ }^{8}$

Scheme 1. The reagent controlled site-selectivity in metalation of $\mathrm{N}$-pivolyl-2-(3methoxyphenyl)ethylamine.

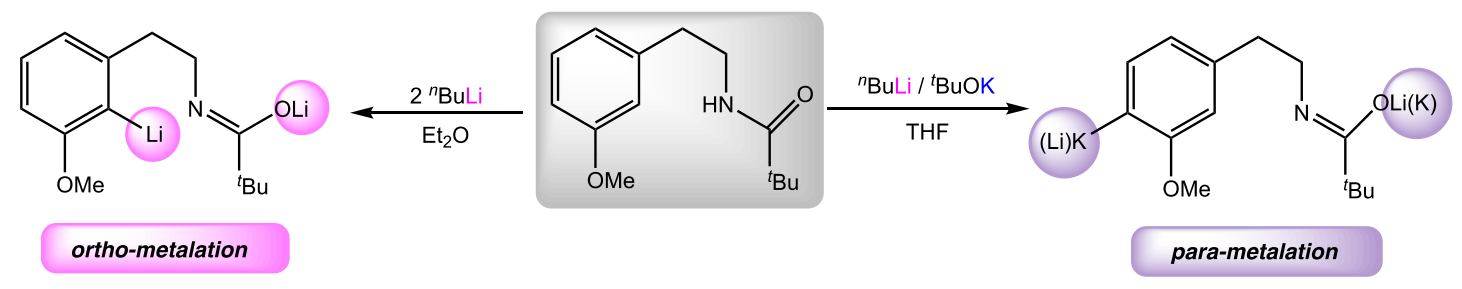

Qualitatively, in terms of relative Brønsted basicity, the binary mixture $\left[\left\{\left({ }^{n} \mathrm{BuLi}\right)\left({ }^{t} \mathrm{BuOK}\right)\right\}_{\mathrm{n}}\right]$ (commonly written as LIC-KOR) exhibits reactivity intermediate between that of $n$-butyllithium and $n$-butylpotassium. In general, organopotassium compounds are orders of magnitude more reactive than their organolithium congeners but the flip side is their reduced stability (autodestruction) and propensity to side reactions, which is why organopotassium compounds on their own are not so useful reagents, especially to non-specialists. The "superbase" designation associated with LIC-KOR originates from its enhanced reactivity compared to $n$-butyllithium, and 
though it is less reactive than $n$-butylpotassium, its reactivity is generally more controllable kinetically and thus LIC-KOR is more utilizable than the separated alkylpotassium compound. Expressing the synergistic effect of LIC-KOR in broad terms, Schlosser notes, "the symbiotic action of two different metals (lithium and potassium) suppresses erratic side reactions".$^{9}$

The fact that the chemistry of LIC-KOR largely revolves around its role as a broad-spectrum base with exceptional metalating properties makes a review of its reactions unnecessary ${ }^{10}$ especially as Mongin and Harrison-Marchand have comprehensively covered the reaction chemistry of LIC-KOR in the recent aforementioned Chemical Reviews article. ${ }^{3,4}$ As a very approximate rule of thumb, if the commercial alkyllithium reagents $n$-butyllithium or $t$-butyllithium do not produce the desired deprotonation or polydeprotonation of a substrate, chemists often turn to LIC-KOR (Scheme 2).

Scheme 2. Contrasting metalation power of ${ }^{n} \mathrm{BuLi}$ and LIC-KOR.

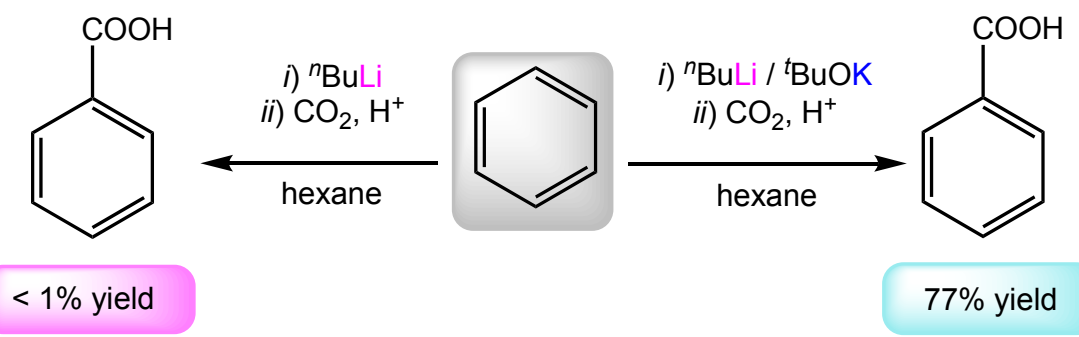

More informative would be evidence that might explain the basis of the synergistic effect. However, despite the fact that LIC-KOR has been utilized in synthetic chemistry since $1967,{ }^{5}$ much uncertainty still persists about its structure(s) with definitive characterisation proving elusive to date. Clues are available from related bimetallic compositions. An early $a b$ initio theoretical study using a simplified computational 
$\left[(\mathrm{MeLi})_{x}(\mathrm{MeOK})_{\mathrm{y}}\right]$ model for LIC-KOR in dinuclear, trinuclear and tetranuclear stoichiometries identified a low-energy, alkoxide-rich cubane structure, $\left[(\mathrm{MeLi})(\mathrm{MeOK})_{3}\right]\left(\right.$ Figure 1). ${ }^{11}$

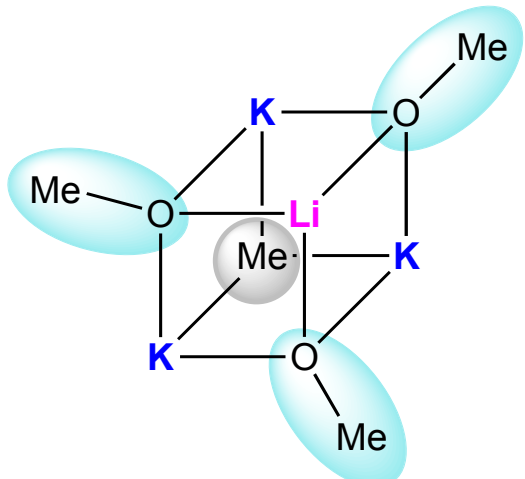

Figure 1. Proposed cubane structure of $\left[(\mathrm{MeLi})(\mathrm{MeOK})_{3}\right]$.

Possessing basicity-enhancing features of long $\mathrm{K}-\mathrm{C}$ contacts, no Li-C contacts and a higher degree of negative charge on the methyl group than in the other aggregates, this structure is close to being a free methyl carbanion carrier and thus a potential model complex for a LIC-KOR metalating agent. Experimental crystallographic evidence for LIC-KOR type structures has been thin on the ground. Until recently the closest LIC-KOR analog to be structurally authenticated was the 1:1 amidolithium:potassium-t-butoxide composite $\left[\left\{{ }^{t} \mathrm{BuN}(\mathrm{H})\right\}_{4}\left({ }^{t} \mathrm{BuO}\right){ }_{4} \mathrm{Li}_{4} \mathrm{~K}_{4}\left(\mathrm{C}_{6} \mathrm{H}_{6}\right)_{n}\right] \quad(\mathbf{1}),{ }^{12}$ though its amido component makes it more relevant to the known but much less utilized LDA-KOR ( $\left.{ }^{i}{ }_{2} \mathrm{NLi} /{ }^{t} \mathrm{BuOK}\right)$ superbase, the structure of which is also unknown. In the crystal 1 is a tetramer featuring dimeric $(\mathrm{LiN})_{2}$ and $(\mathrm{KO})_{2}$ rings in a captivating carousel arrangement (Figure 2). 


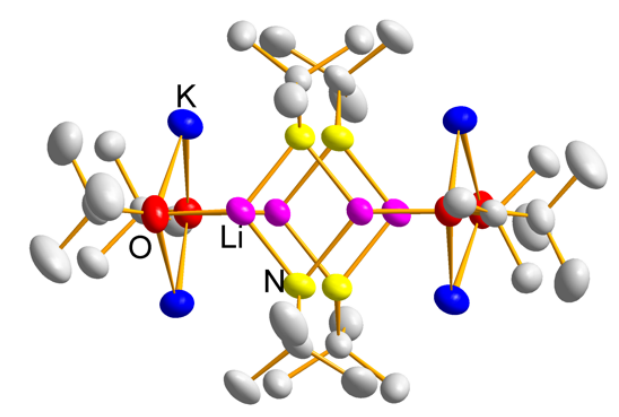

Figure 2. Core structure of $\left[\left\{^{t} \mathrm{BuN}(\mathrm{H})\right\}_{4}\left({ }^{t} \mathrm{BuO}\right){ }_{4} \mathrm{Li}_{4} \mathrm{~K}_{4}\left(\mathrm{C}_{6} \mathrm{H}_{6}\right)_{n}\right], 1$.

It too can be classed as a superbase in the sense that it deprotonates toluene in contrast to the inertness of its separated lithium amide and potassium alkoxide components. Other informative crystallographic studies of superbase-related constitutions have appeared recently. The discrete dilithium-tetrapotassium cluster complex of formula $\left[(\mathrm{PhK})_{4}(\mathrm{PhLi})\left({ }^{t} \mathrm{BuOLi}\right)(\mathrm{THF})_{6}\left(\mathrm{C}_{6} \mathrm{H}_{6}\right)_{2}\right]$ (2) is a postmetalation crystalline product of the reaction between LIC-KOR and excess benzene in THF at low temperature (Scheme 3). ${ }^{13}$

Scheme 3. Preparation of polymeric $\left[(\mathrm{PhK})_{4}(\mathrm{PhLi})\left({ }^{t} \mathrm{BuOLi}\right)(\mathrm{THF})_{6}\left(\mathrm{C}_{6} \mathrm{H}_{6}\right)_{2}\right] 2$ and its reactivity with toluene. 


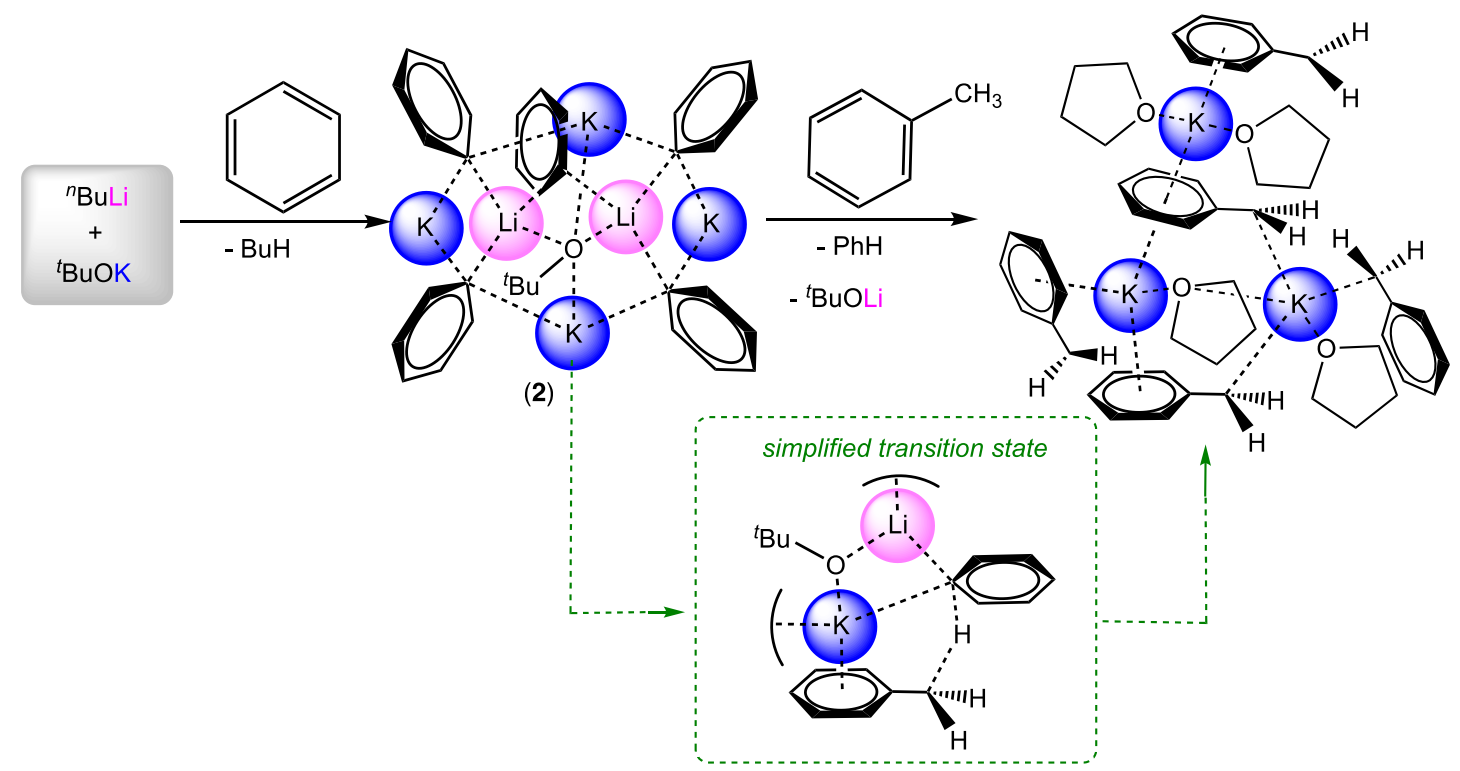

The most interesting structural feature of this arylpotassium-rich compound is the hard-soft bonding distinction of the Li/K partnership. Its Li atoms occupy the "hard" core coordinated by one ${ }^{t} \mathrm{BuO}$ and three $\mathrm{Ph}$ anions with in-plane $\mathrm{Ph}-\mathrm{Li}$ interactions signifying significant $\sigma$ character. In contrast, its $\mathrm{K}$ atoms occupy the "soft" periphery around this core, in an eight-atom $(\mathrm{KC})_{4}$ ring arrangement with out-of-plane $\pi-\mathrm{Ph}-\mathrm{K}$ interactions. Isolated $\mathbf{2}$ was found to act as a synergistic deprotonating agent itself by metalating toluene to form benzylpotassium. A basic mechanism was postulated for this reaction featuring a four-membered $\left[\mathrm{K}(\mathrm{Ph}) \mathrm{Li}\left({ }^{t} \mathrm{BuO}\right)\right]$ ring that engages with toluene via its $\mathrm{K}-\mathrm{C}$ edge in the transition state (Scheme 3 ). The synergistic origin of this toluene deprotonation reaction was attributed to pre-coordination of the $\pi$ electrons of the arene to the soft potassium cation combined with the hard lithium cation anchoring the anions and thus limiting the size of the aggregate and concomitantly increasing the solubility of the base. Good solubility was also a key factor in enabling NMR spectroscopic and X-ray crystallographic characterization of mixtures of $\left[\mathrm{Li}_{x} \mathrm{~K}_{y} \mathrm{~Np}_{z}\left({ }^{t} \mathrm{BuO}\right)_{x+y-z}\right]$, in which the higher neo-pentyl $\left(\mathrm{CH}_{2}{ }^{t} \mathrm{Bu}, \mathrm{Np}\right)$ homologue replaces the butyl group of the classical Lochmann-Schlosser superbase. ${ }^{14}$ The basic structure 
of the mixture comprises a square plane of four potassium centers $\left[\mathrm{K}_{4}\right]^{4+}$, capped from above and below by charge-balancing $\left[\mathrm{NpLi}\left({ }^{t} \mathrm{BuO}\right){ }_{2} \mathrm{LiNp}\right]^{2-}$ dianions positioned staggered to each other. (Figure 3).

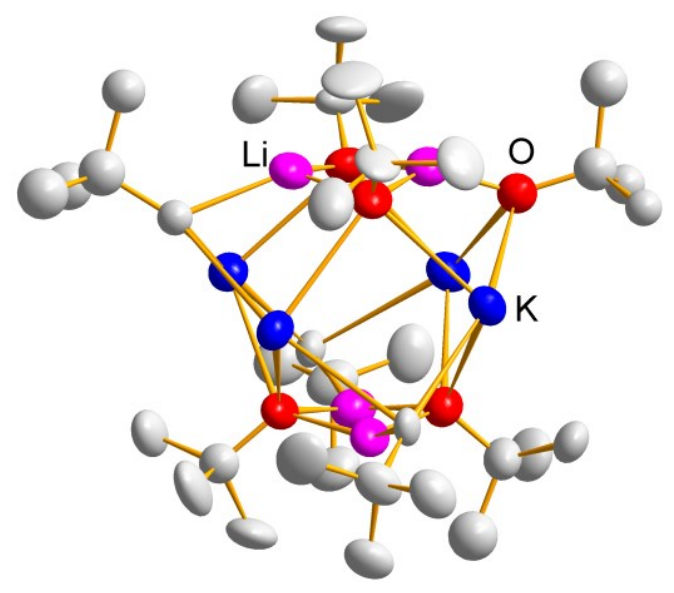

Figure 3. Molecular structure of $\left[\mathrm{Li}_{4} \mathrm{~K}_{4} \mathrm{~Np}_{3}\left(\mathrm{O}^{t} \mathrm{Bu}\right)_{5}\right] 3$.

The eight metal centers fill the corners of a truncated tetrahedron, generating four $\mathrm{LiK}_{2}$ triangles capped mainly by $\mathrm{Np}$ groups and four $\mathrm{Li}_{2} \mathrm{~K}_{2}$ trapeziums capped exclusively by ${ }^{t} \mathrm{BuO}$ groups. By changing the stoichiometry of the NpLi/ ${ }^{t} \mathrm{BuOK}$ mixture, crystals of similar structure could be isolated ranging from $\mathrm{Np}$-free, all-alkoxide $\left[\mathrm{Li}_{4} \mathrm{~K}_{4}\left({ }^{t} \mathrm{BuO}\right)_{8}\right]$ to $\left[\mathrm{Li}_{4} \mathrm{~K}_{4} \mathrm{~Np}_{3}\left({ }^{t} \mathrm{BuO}\right)_{5}\right](3)$. In solution such compounds appear to be in equilibrium, while higher $\mathrm{Np}$ content results in reduced stability and eventually to separation of $\mathrm{Li}$ and $\mathrm{K}$ species to yield charge-separated structures with a $\left[\mathrm{Li}_{4}\left({ }^{t} \mathrm{BuO}\right)_{3}\right]^{+}$ cation paired with either a $\left[\mathrm{K}_{3} \mathrm{~Np}_{3}\left({ }^{t} \mathrm{BuO}\right)\right]^{-}$or $\left[\mathrm{K}_{3} \mathrm{~Np}_{4}\right]^{-}$anion. Significantly, the aforementioned all-alkoxide $\left[\mathrm{Li}_{4} \mathrm{~K}_{4}\left({ }^{t} \mathrm{BuO}\right)_{8}\right]{ }^{15}$ and its congeners $\left[\mathrm{Li}_{4} \mathrm{M}_{4}\left({ }^{t} \mathrm{BuO}\right)_{8}\right],(\mathrm{M}=$ $\mathrm{Na}, \mathrm{Rb}, \mathrm{Cs}$ ) reported in $2000,{ }^{16}$ all adopt similar "breastplate" structures for which it was noted that "an inherently stable contacted triple ion sandwich comprising a dianion-tetramonocation-dianion arrangement, the breastplate motif is likely to be more widely applicable within heterometallic structural chemistry than so far 
recognized". This prediction seems corroborated, as the new neo-pentyl derivatives in effect are isostructural with this homologous series. Though the structure(s) of the LIC-KOR superbase remains to be formally elucidated, extrapolation of this new evidence points to it adopting a related breastplate type arrangement (Figure 4) and undergoing similar equilibria processes to the neo-pentyl system.

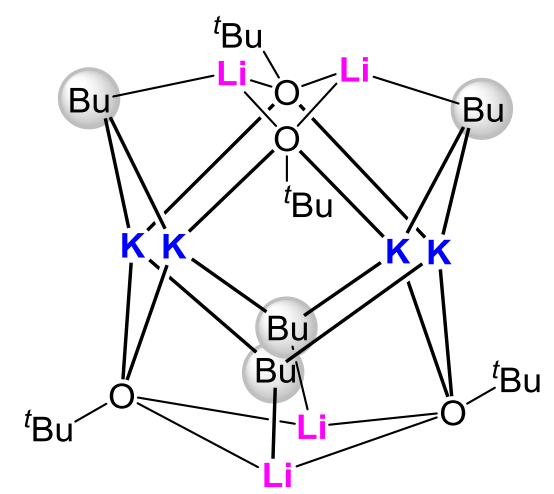

Figure 4. Proposed breastplate type arrangement of LIC-KOR.

Turning to recent novel reactions of LIC-KOR, 2-oxazolines possessing an adjacent benzyl ether function surprisingly undergo cyclisation with ring opening of the oxazoline to give 2-aryl-3-aminobenzofurans in good yield (Scheme 4). ${ }^{17}$

Scheme 4. Contrasting outcomes of metalation of 2-oxazoline with ${ }^{n}$ BuLi or LIC-KOR.<smiles>CC1(C)COC(c2ccccc2C(O)c2ccccc2)=N1</smiles><smiles>CC1(C)COC(c2ccccc2OCc2ccccc2)=N1</smiles><smiles>CC(C)(CO)Nc1c(-c2ccccc2)oc2ccc(C(F)(F)F)cc12</smiles>
In contrast, ${ }^{n} \mathrm{BuLi}$ in THF mainly gives the Wittig rearrangement product highlighting the divergent synergistic chemistry of the mixed-metal reagent. LIC-KOR has also proved the key to establishing a new type of reactivity for $\alpha, \mathrm{N}$-diarylnitrones. ${ }^{18}$ 
Generated by LIC-KOR metalation of an $\alpha, \beta$-unsaturated acetal, metalated-1alkoxydienes react with nitrones, following which a domino process takes place, whereby an E1cb elimination reaction results enabling the stereoselective synthesis of 2-ethoxy-N-phenyl-1-arylpenta-2,4-dien-1-imines (Scheme 5).

Scheme 5. Proposed mechanism for formation of $\alpha, N$-diphenyldienyloxyimine.

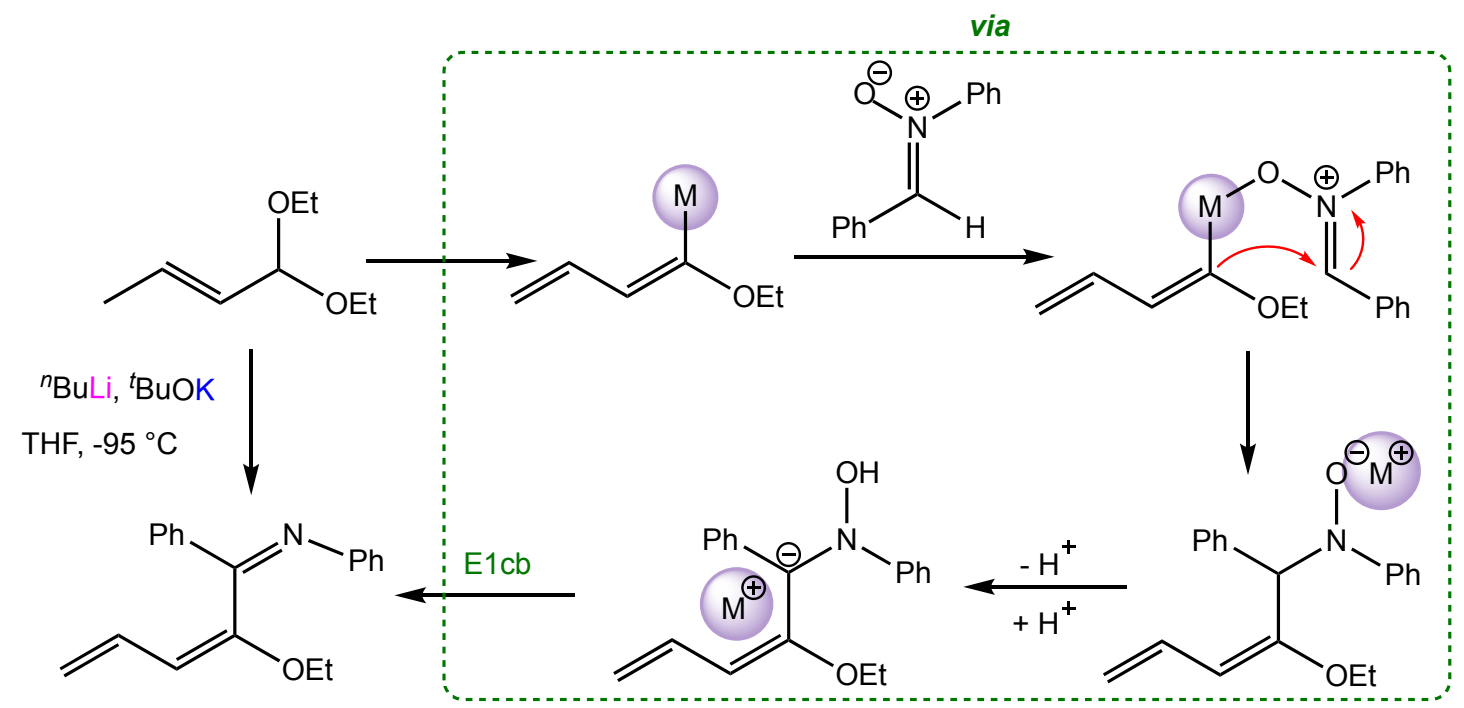

The regioselective metalating properties of LIC-KOR (here ${ }^{t} \mathrm{BuLi}-\mathrm{Bu}^{t} \mathrm{OK}$ ) have been extended to macromolecular systems through metalation of poly(spirophosphazene) $\left[\left\{\mathrm{N}=\mathrm{P}\left(\mathrm{O}_{2} \mathrm{C}_{12} \mathrm{H}_{8}\right)\right\}_{n}\right] .{ }^{19}$ Optimisation studies found that a fourfold excess of the base in THF at $-78^{\circ} \mathrm{C}$ gave the highest regioselective substitution at the ortho position of the biphenoxy scaffold. Following metalation, electrophilic units (-BPin, -SiMe,$-\mathrm{PPh}_{2}$ ) were cleanly substituted onto the polyphosphazenes, promoting systematic changes in their solubility, electronic properties, and $\mathrm{Tg}$ values. Confirming the synergistic action of this metalation, it was noted that ${ }^{t} \mathrm{BuLi}$ on its own did not significantly lithiate the aromatic rings (Scheme 6). 
Scheme 6. Metalation of polyphosphazene by LIC-KOR and its failure with ${ }^{\mathrm{t}} \mathrm{BuLi}$.

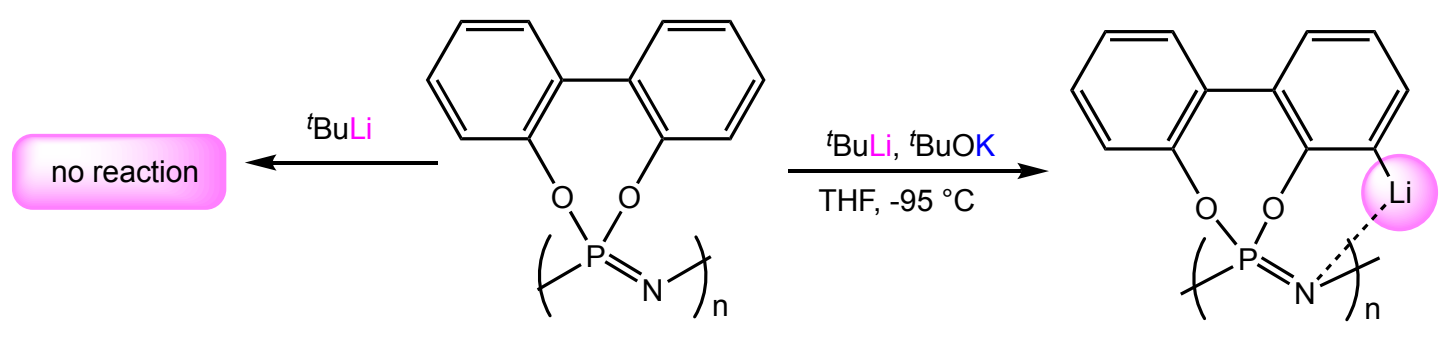

In a variation of the classic superbase, tetramethypiperidine $[T M P(H)]$ was added to LIC-KOR to generate a three-component system of ${ }^{n} \mathrm{BuLi} /{ }^{t} \mathrm{BuOK} / \mathrm{TMP}(\mathrm{H})$ (so called LiNK metalation conditions). A clear synergistic effect is in operation with this triad system when used in THF at $-78{ }^{\circ} \mathrm{C}$, as while LIC-KOR exhibits no selectivity towards 2,5-dimethylanisole producing a cocktail of $o$-aryl, $o$-benzylic, and $m$-benzylic isomers, the introduction of the third component $\mathrm{TMP}(\mathrm{H})$ leads to excellent selectivity for the $m$-benzylic site (Scheme 7). ${ }^{20}$

Scheme 7. Selective benzylic metalation of 2,5-dimethylanisole.
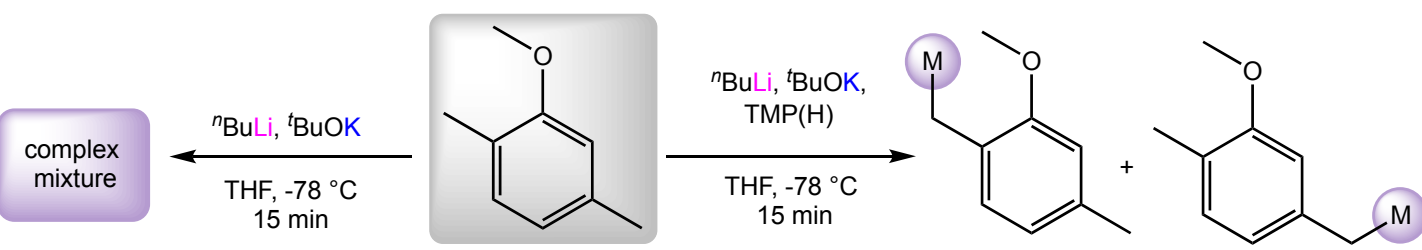

$80 \%$ yield, 5:95 ratio

Moreover, subjecting $o^{-}, m-$, and $p$-OMOM substituted toluene substrates to LIC-KOR produced highly selective $o$-metalation with over $93 \%$ incorporation of deuterium via $\mathrm{CD}_{3} \mathrm{OD}$. On the other hand, following metalation with ${ }^{n} \mathrm{BuLi} /{ }^{t} \mathrm{BuOK} / \mathrm{TMP}(\mathrm{H})$, benzylic deuteriated products with insignificant $0-\mathrm{D}$ incorporation were obtained (Scheme 8).

Scheme 8. Selective metalation of OMOM-substituted toluenes. 
<smiles>COc1ccccc1-c1ccccc1</smiles>

i) ${ }^{n} \mathrm{BuLi},{ }^{\mathrm{B}} \mathrm{BuOK}$

$\mathrm{THF},-78^{\circ} \mathrm{C}$

$$
15 \mathrm{~min}
$$

ii) $\mathrm{CD}_{3} \mathrm{OD}$
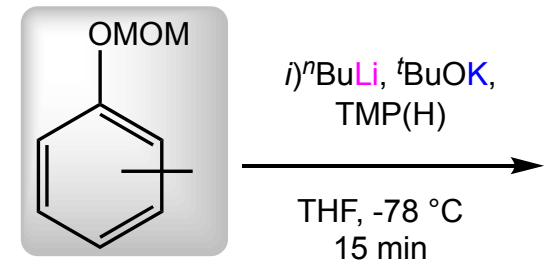

ii) $\mathrm{CD}_{3} \mathrm{OD}$

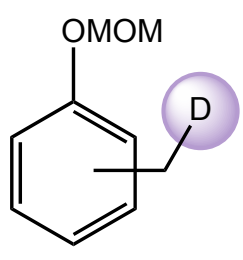

$20-88 \%$ yield

However, the deuterated product of the $p$-OMOM isomer could only be obtained in low yield reflecting the lower acidity of the benzylic $\mathrm{H}$ atoms in this 4-substituted species. Note that in LiNK chemistry, the amine TMP $(\mathrm{H})$ becomes the amide TMP and induces an in situ anion migration from o-aryl sites to benzylic sites as evidenced from NMR studies of LIC-KOR solutions with catalytic TMP(H) employing $o$, and $m$-OMOM substituted toluene substrates. This LiNK system can also selectively metalate a series of substituted toluenes at the lateral $\mathrm{CH}_{3}$ position to generate benzylic derivatives that in turn can undergo transmetalation to silicon on treatment with trimethylsilylchloride in a general one-pot procedure for synthesis of substituted benzyltrimethylsilanes, $\mathrm{ArCH}_{2} \mathrm{Si}(\mathrm{Me})_{3 .}{ }^{21}$ Stable on the bench unlike polar metal benzyl compounds, these lower polarity benzyl species function as surrogate benzyl anions taking part in a series of addition reactions to both non-enolizable and enolizable carbonyls (Scheme 9).

Scheme 9. LiNK metalation route to bench stable benzyl-TMS followed by subsequent addition to aldehydes and ketones.

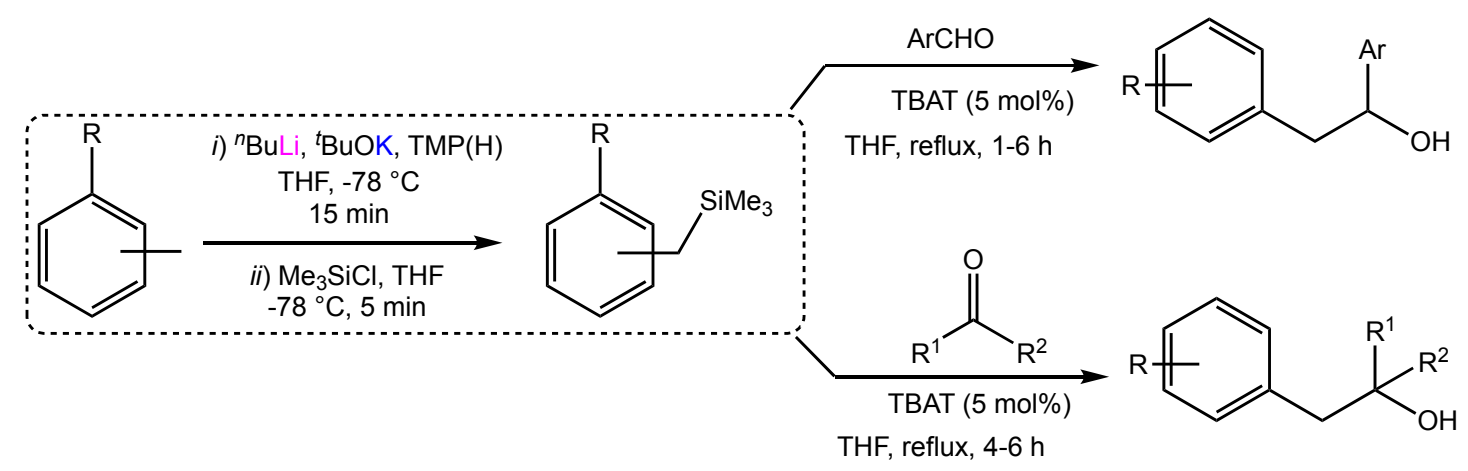


$\alpha, \alpha$-Bis(trimethylsilyl)toluenes made by the LiNK method have also been converted to alkene derivatives via stereoselective Peterson olefination reactions of broad scope using substituted $N$-benzylideneanilines as electrophiles. ${ }^{22}$ LiNK metalation has also been utilized in a two-step synthesis of mono- and di-carboxy planar chiral [2.2]metacyclophanes starting from $m$-xylenes. Compounds of this type normally require lengthier syntheses. The most difficult step from a metalation perspective was a fourfold metalation of the dibenzoic acid, but this was smoothly accomplished via two equivalents of LIC-KOR for the challenging benzylic metalations (aryl or methylene metalation could potentially interfere) and two additional equivalents of $n$-butyllithium for the routine carboxylic acid metalations (Scheme 10). ${ }^{23}$

Scheme 10. Selective dibenzylic metalation of dibenzoic acid affording tetrametalated species. Competing potential metalation sites labelled with red arrows.<smiles>Cc1ccc(C(=O)O)c(CC(C)(C)C)c1</smiles><smiles>O=C([O-])c1ccc(C(=O)[O-])c(CCc2cc(CCc3ccccc3)ccc2C(=O)[O-])c1</smiles>

Oxidative coupling via 1,2-dibromoethane completed the ring closure to the cyclophanes, which are of interest due to their potential utility as chiral catalysts and ligands. This LiNK-based methodology was subsequently upgraded from a racemic two-step synthesis to a three-step asymmetric synthesis by introducing an enantioselective step achieved via an ortho-aryl metalation using ${ }^{\mathrm{s}} \mathrm{BuLi}(-)$-sparteine in 
diethyl ether at $-40{ }^{\circ} \mathrm{C} .{ }^{24}$ Further work has established that the LiNK methodology is not limited to a polar THF medium but can also be successful in hydrocarbon media. ${ }^{25}$ Through deuterium labeling, it was proven that as in THF solution it is necessary to mix together all three reagents ${ }^{n} \mathrm{BuLi}, \mathrm{KO}^{t} \mathrm{Bu}$, and $\mathrm{TMP}(\mathrm{H})$ to ensure good reactivity and selectivity. Comparison with the aforementioned reactions that produced benzyltrimethylsilane and $\alpha, \alpha$ - bis(trimethylsilyl)toluene reagents, which in turn can be used for nucleophilic addition and Peterson olefination applications, showed that hydrocarbon media (for example, heptane or cyclohexane at $0^{\circ} \mathrm{C}$ ) were in general as effective as THF. The benzyl metalated products can also undergo directed oxidative C-C couplings via 1,2-dibromoethane as for example in the one-step synthesis of the natural product Brittonin A (Scheme 11).

Scheme 11. Synthesis of Brittonin $\mathrm{A}$ by $\mathrm{sp}^{3} \mathrm{C}-\mathrm{sp}^{3} \mathrm{C}$ coupling of carbon nucleophiles generated by LiNK metalation.
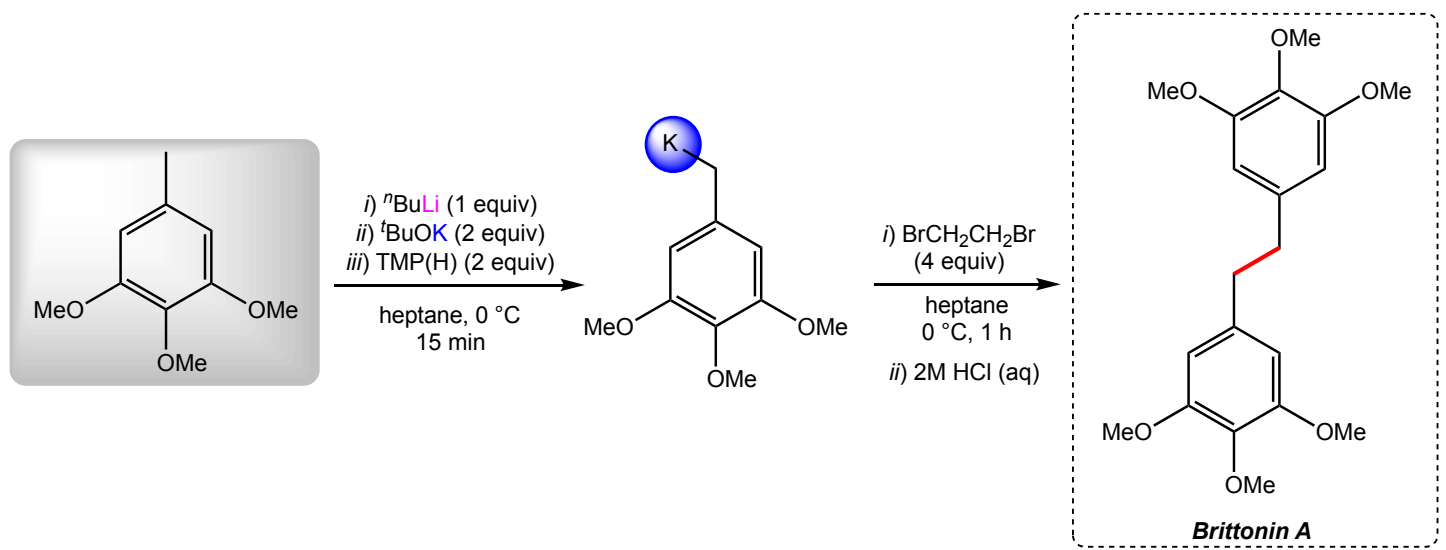

\subsubsection{Other Alkali Metal - Alkali Metal Systems}

Though compositions containing more than one alkali metal are common in many of them the alkali metals are present merely as counter ions so their presence does not have a significant bearing on the chemistry of interest. This applies to a wide variety 
of areas such as host-guest, MOF and polyoxometalate chemistries so in general these areas are excluded from this review. Of course, the vast majority of alkali metal chemistry is aqueous based so that too is outside the remit of this article, which deals specifically with synergistic effects in polar organometallic chemistry that normally require anaerobic, anhydrous conditions.

Nobel laureate Wittig coined the term "ate", short for metalate, to refer to the situation that a bimetallic complex could exhibit a different reactivity to that of its separated single-metal combinations. In a seminal paper from 1951 he introduced the mixed-metal magnesiate complex "LiMgPh 3 " reporting that its reaction with benzalacetophenone gives a product of predominately 1,4-addition, while the monometallic precursor LiPh gives predominately a 1,2-addition product. ${ }^{26}$ This was clearly a very early recognition of a synergistic effect, which Wittig rationalized in terms of an anionic activation, since the ate complex formally contains a metal as part of a complex anion with the negative charge activating "anionically" the ligands surrounding this metal through an inductive effect. ${ }^{27}$ Interestingly mixed lithium sodium polar organometallic chemistry containing a set of organic ligands only started in 1958 when Wittig introduced "diphenyllithium-sodium", formulated as $[\mathrm{Na}(\mathrm{Ph}) \mathrm{Li}(\mathrm{Ph})]$, and reported the synergistic observation that this mixed-metal compound could stabilize phenylsodium in any desirable excess in ether solution; whereas phenylsodium alone decomposes rapidly in the same medium. ${ }^{28,29} \mathrm{~A}$ diamine-solvated modification $\left[\{\mathrm{Na}(\mathrm{TMEDA})\}_{3}\right]\left[\mathrm{Li}(\mathrm{Ph})_{4}\right]$ (4) was crystallographically characterized in 1988 and found to be an ate complex with a distorted tetrahedral $\left[\mathrm{Li}(\mathrm{Ph})_{4}\right]^{-}$trianion held together by three $\left[\left\{\mathrm{Na}(\mathrm{TMEDA}\}_{3}\right]^{+}\right.$cations (Figure 5$) \cdot{ }^{30}$ 


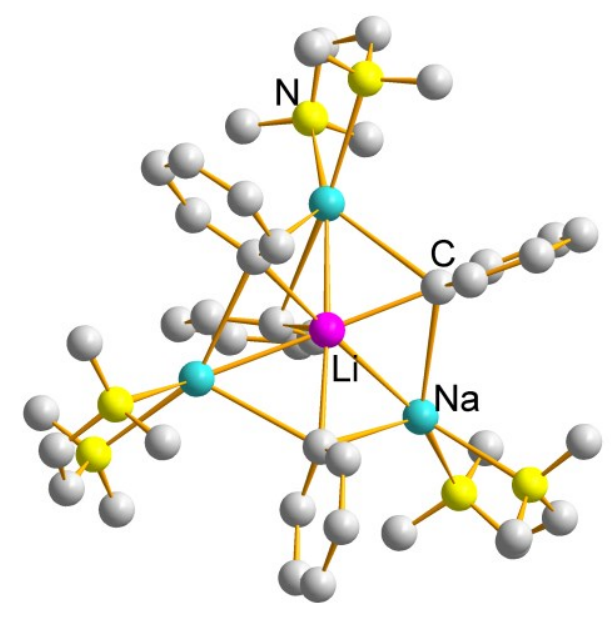

Figure 5. Molecular structure of $\left[\{\mathrm{Na}(\mathrm{TMEDA})\}_{3}\right]\left[\mathrm{Li}(\mathrm{Ph})_{4}\right], 4$.

Two years earlier appeared the crystal structure of the guanidinate complex $\mathrm{LiNa}_{3}(\mathrm{HMPA})_{3}\left[\mathrm{~N}=\mathrm{C}\left(\mathrm{NMe}_{2}\right)_{2}\right](5),{ }^{31}$ also having a 1:3, Li:Na stoichiometry but this time distinguished by a pseudo-cubic LiNaz mixed-metal core, while a double cubic facesharing arrangement was seen in the related ketimide $\left[\left\{\mathrm{Li}_{4} \mathrm{Na}_{2}\left[\mathrm{~N}=\mathrm{C}(\mathrm{Ph})\left({ }^{t} \mathrm{Bu}\right)\right\}_{6}\right](6\right.$, Figure 6)..$^{32}$
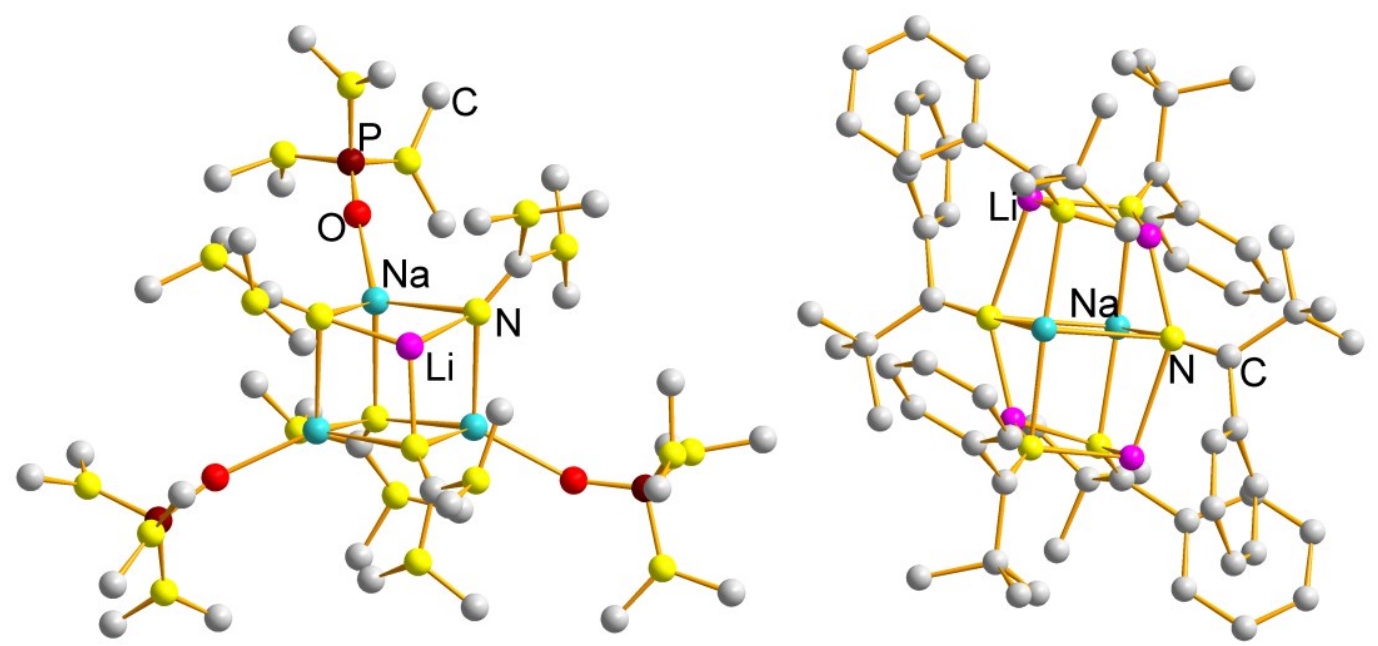

Figure 6. Molecular structures of $\mathrm{LiNa}_{3}(\mathrm{HMPA})_{3}\left[\mathrm{~N}=\mathrm{C}\left(\mathrm{NMe}_{2}\right)_{2}\right] \quad$ (5, LHS) and $\left[\left\{\mathrm{Li}_{4} \mathrm{Na}_{2}\left[\mathrm{~N}=\mathrm{C}(\mathrm{Ph})\left({ }^{\mathrm{t} B u}\right)\right\}_{6}\right](6, \mathrm{RHS})\right.$. 
Viewed as constructed from the face-to-face association of dimeric $(M-N)_{2}$ rings these two structures fit well with the ring-stacking principle established for a large variety of organolithium and lithium amide structures. ${ }^{33}$

Beautiful examples of the power of alkali metal - alkali metal cooperativity to enable the self-assembled construction of an unprecedented class of organometallic supramolecule has come in corannulene $\left(\mathrm{C}_{20} \mathrm{H}_{10}\right)$ chemistry. ${ }^{34,35}$ Corannulene, the smallest curved section of the $\mathrm{C}_{60}$ fullerene is easily reduced by lithium metal forming a crystallographically characterized sandwich complex with a pentanuclear $\left[\mathrm{Li}_{5}\left(\mathrm{C}_{20} \mathrm{H}_{10}{ }^{4-}\right)_{2}\right]^{3-}$ trianion. ${ }^{36}$ The 5 -membered ring hub site in this sandwich is unoccupied since there is insufficient space for a sixth Li cation. However, this site was filled and maximum occupancy of the bowl-shaped corannulene rings was achieved by using lithium potassium mixtures to produce crystalline complexes containing the triple-decker anions $\left[\left(\mathrm{C}_{20} \mathrm{H}_{10}\right)^{4-}\left(\mathrm{LiK}_{5}\right)^{6+}\left(\mathrm{C}_{20} \mathrm{H}_{10}\right)^{4-}\right]^{2-}$ and $\left[\left(\mathrm{C}_{20} \mathrm{H}_{10}\right)^{4-}\left(\mathrm{Li}_{3} \mathrm{~K}_{3}\right)^{6+}\left(\mathrm{C}_{20} \mathrm{H}_{10}\right)^{4-}\right]^{2-}$. Here the presence of the larger alkali metal pushes the $C_{20}$ rings further apart to enable a $\mathrm{Li}$ cation to enter the hub site, a movement the authors' pictured imaginatively as a clamshell opening (Figure 7).

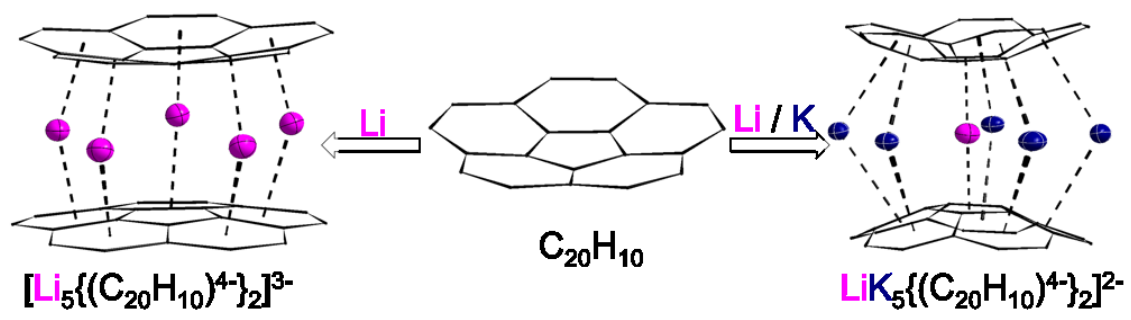

Figure 7. Supramolecular aggregates with a) $\mathrm{Li}_{5}$ and b) $\mathrm{LiK}_{5}$-cores sandwiched between two corannulene rings. 
This triple-decker tetra-reduced corannulene family was extended to mixed lithiumrubidium clusters with $\mathrm{Li}_{3} \mathrm{Rb}_{2}, \mathrm{Li}_{3} \mathrm{Rb}_{3}$ or $\mathrm{LiRb}_{5}$ cores sandwiched between two $\mathrm{C}_{20} \mathrm{H}_{10}{ }^{4-}$ rings (Figure 8$) \cdot{ }^{37}$

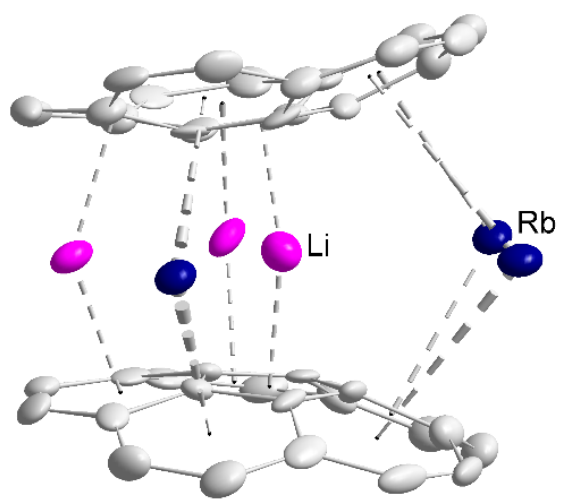

Figure 8. Core sandwich structure of $\left[\left\{\mathrm{Li}_{3} \mathrm{Rb}_{3}\left(\mathrm{C}_{20} \mathrm{H}_{10}{ }^{4-}\right)_{2}\right\}^{2-}\right]$.

Again, in each case a Li cation fills the hub-cavity between the pair of $\mathrm{C}_{20} \mathrm{H}_{10}{ }^{4-}$ rings leading to a record high negative ${ }^{7} \mathrm{Li}$ NMR shift (up to $-25 \mathrm{ppm}$ ). Penta-decker sandwich complexes could be accessed via mixed cesium-potassium reduction of $\mathrm{C}_{20} \mathrm{H}_{10}{ }^{38}$ Mixtures comprising potassium, cesium, and corannulene in a 10:3.5:1 stoichiometric ratio produced the first triply-reduced corannulene radical species in $\left[\mathrm{Cs}^{+} / /\left(\mathrm{C}_{20} \mathrm{H}_{10}{ }^{3-}\right) / 4 \mathrm{~K}^{+} /\left(\mathrm{C}_{20} \mathrm{H}_{10}{ }^{3-}\right) / / \mathrm{Cs}^{+}\right]$in which two corannulene trianion decks encapsulate a rectangle of four potassium ions, with the exterior bowl cavities occupied exclusively by one cesium ion (Figure 9). 


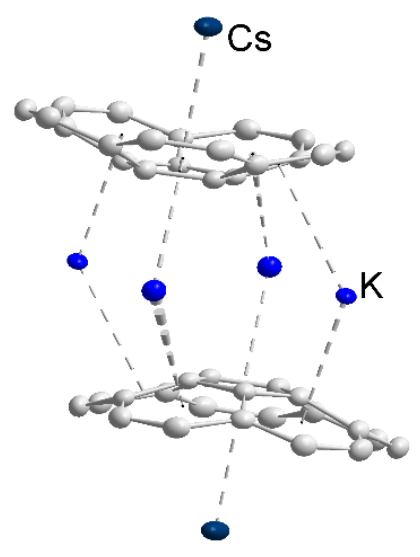

Figure 9. Core sandwich structure of $\left[\left\{\mathrm{K}_{2} \mathrm{Cs}\left(\mathrm{C}_{20} \mathrm{H}_{10}\right)^{3-}\right\}_{2}\right]$.

Magnetic data point to a weak antiferromagnetic interaction between two trianionradicals through the potassium deck in the sandwich. A clear synergistic mixed-metal effect is in operation here as the single metal Li-executed reduction of corannulene rapidly advances to the further reduced tetra species, whereby the mixed metal trianionic species is isolable. Moreover, incorporating different sized alkali metal decks within the sandwich facilitates tuning of the coupling of $\mathrm{C}_{20} \mathrm{H}_{10}{ }^{-3-}$ radicals from anti-ferromagnetic to ferromagnetic in character. These reduced corannulenes belong to a class of non-planar carbonaceous aromatic compounds, which includes fullerenes and carbon nanotubes that show promise as lightweight materials for battery and electronic technologies.

Treating one equivalent of the bulky aminoquinoline $\left[\mathrm{HN}\left(8-\mathrm{C}_{9} \mathrm{H}_{6} \mathrm{~N}\right)\left(\mathrm{Si}^{t} \mathrm{BuMe}_{2}\right)\right]$ with a half equivalent each of $n$-butyllithium and $n$-butylsodium has produced a heterometallic lithium-sodium amide $\left[\mathrm{Li}\left\{\mathrm{N}\left(8-\mathrm{C}_{9} \mathrm{H}_{6} \mathrm{~N}\right)\left(\mathrm{Si}^{t} \mathrm{BuMe}_{2}\right)\right\}_{2} \mathrm{Na}(\mathrm{TMEDA})\right]$ (7) instead of a simple binary mixture of the lithium amide and sodium amide. ${ }^{39}$ Its structure in the crystal is predictable having a discrete four-membered $\left[\mathrm{M}\right.$ (amido) $\mathrm{N}_{2}$ 
ring of a type commonly found in alkali metal chemistry, with lithium made up to 4coordinate by the neutral $\mathrm{N}$ atoms of the quinoline, while sodium forms a chelate with TMEDA to attain the same tetrahedral geometry (Figure 10).

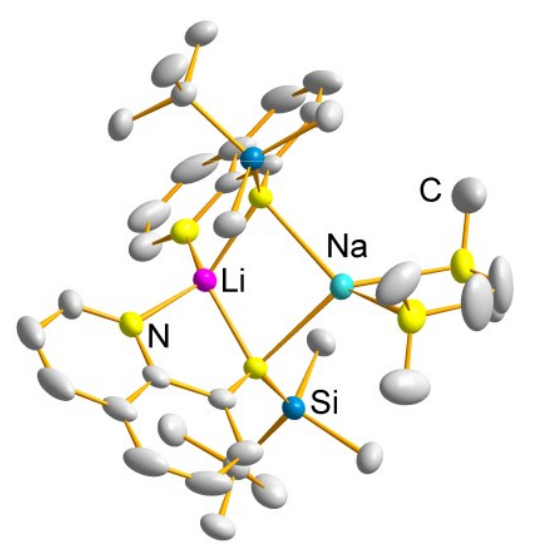

Figure 10. Molecular structure of $\left.\mathrm{Li}\left\{\mathrm{N}\left(8-\mathrm{C}_{9} \mathrm{H}_{6} \mathrm{~N}\right)\left(\mathrm{Si}^{t} \mathrm{BuMe}{ }_{2}\right)\right\}_{2} \mathrm{Na}(T M E D A)\right], 7$.

A study investigating combining Group $1(\mathrm{M}=\mathrm{Na}, \mathrm{K})$ with Group 14 [E = Ge(II), Sn(II)] ions accommodated in adducts between cyclopentadienides MCp and tris(alkoxide) $\mathrm{ME}\left(\mathrm{O}^{t} \mathrm{Bu}\right)_{3}$ salts, produce some interesting synergistic outcomes. On mixing $\mathrm{KCp}$ with $\mathrm{NaGe}\left(\mathrm{O}^{t} \mathrm{Bu}\right)_{3}$ in THF solution an ion exchange takes place to give $\mathrm{NaCp}$ and $\mathrm{KGe}\left(\mathrm{O}^{t} \mathrm{Bu}\right)_{3}$, showing that the tridentate germanate $\left[\mathrm{Ge}\left(\mathrm{O}^{t} \mathrm{Bu}\right)_{3}\right]^{-}$favors the larger, softer potassium to sodium. ${ }^{40}$ However, instead of generating two discrete entities, these complexes combine to form a zig-zag chain polymeric adduct $\left[\mathrm{NaCp}(\mathrm{THF}) \cdot \mathrm{KGe}\left(\mathrm{O}^{t} \mathrm{Bu}\right)_{3}\right]$ (8, Scheme 12).

Scheme 12. Synthesis and structure of $\left[\mathrm{NaCp}(\mathrm{THF}) \cdot \mathrm{KGe}\left(\mathrm{O}^{t} \mathrm{Bu}\right)_{3}\right](\mathbf{8})$. 


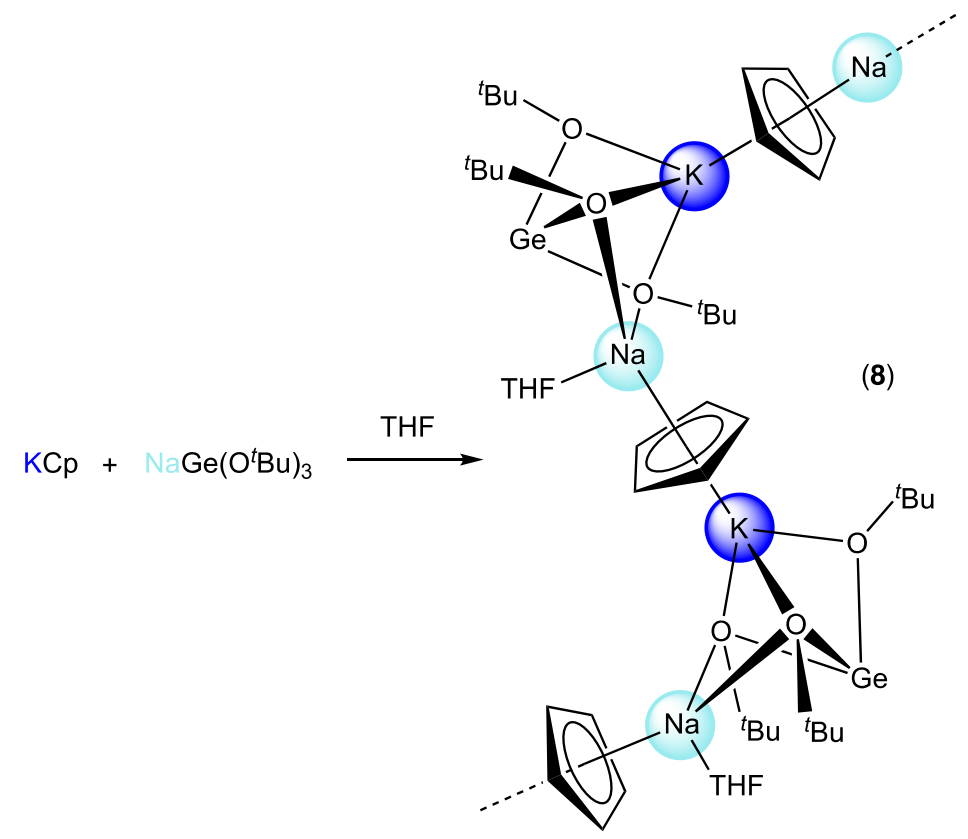

This type of reaction can be likened to trans-metal-trapping (see section 2.4.4), where the transmetalation stops at an intermediate stage to generate a heterobimetallic sodium-potassium structure $\left[\mathrm{NaCp}(\mathrm{THF}) \mathrm{KGe}\left(\mathrm{O}^{t} \mathrm{Bu}\right)_{3}\right]$. When starting with $\mathrm{NaCp}$ and the stannate $\mathrm{KSn}\left(\mathrm{O}^{t} \mathrm{Bu}\right)_{3}$, an isostructural intercalation polymer is obtained $\left[\mathrm{NaCp}(\mathrm{THF}) \mathrm{KSn}\left(\mathrm{O}^{t} \mathrm{Bu}\right)_{3}\right]$ (9) with $\mathrm{Sn}$ replacing $\mathrm{Ge}$ (Scheme 13). Highlighting the differences that can occur by using a different alkali metal combination, when KCp reacts with $\mathrm{KSn}\left(\mathrm{O}^{t} \mathrm{Bu}\right)_{3}$ in $\mathrm{THF}$, the polymer chain $(\mathbf{1 0})$ resembles more the undulating chain structure of $\mathrm{KCp}$ but with additional $\mathrm{K}(\mathrm{THF})_{2} \mathrm{Sn}\left(\mathrm{O}^{\mathrm{t}} \mathrm{Bu}\right)_{3}$ units occupying the obtuse clefts of the chain (Scheme 13).

Scheme 13. Syntheses of $\left[\mathrm{NaCp} \cdot(\mathrm{THF}) \mathrm{KSn}\left(\mathrm{O}^{t} \mathrm{Bu}\right)_{3}\right](\mathbf{9})$ and $\left[\mathrm{KCp}(\mathrm{THF})_{2} \cdot \mathrm{KSn}\left(\mathrm{O}^{t} \mathrm{Bu}\right)_{3}\right](\mathbf{1 0})$. 


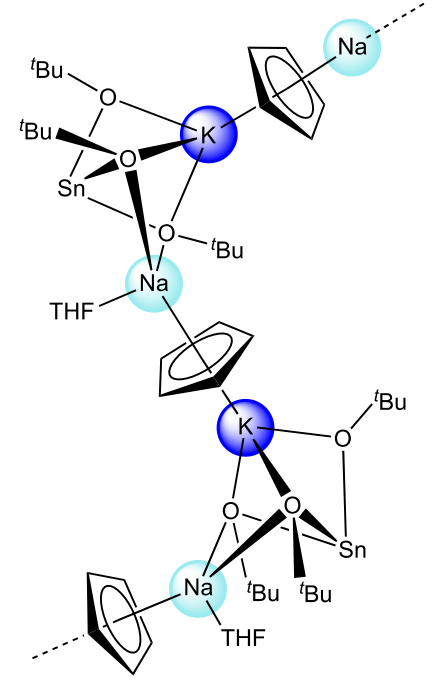

(9)

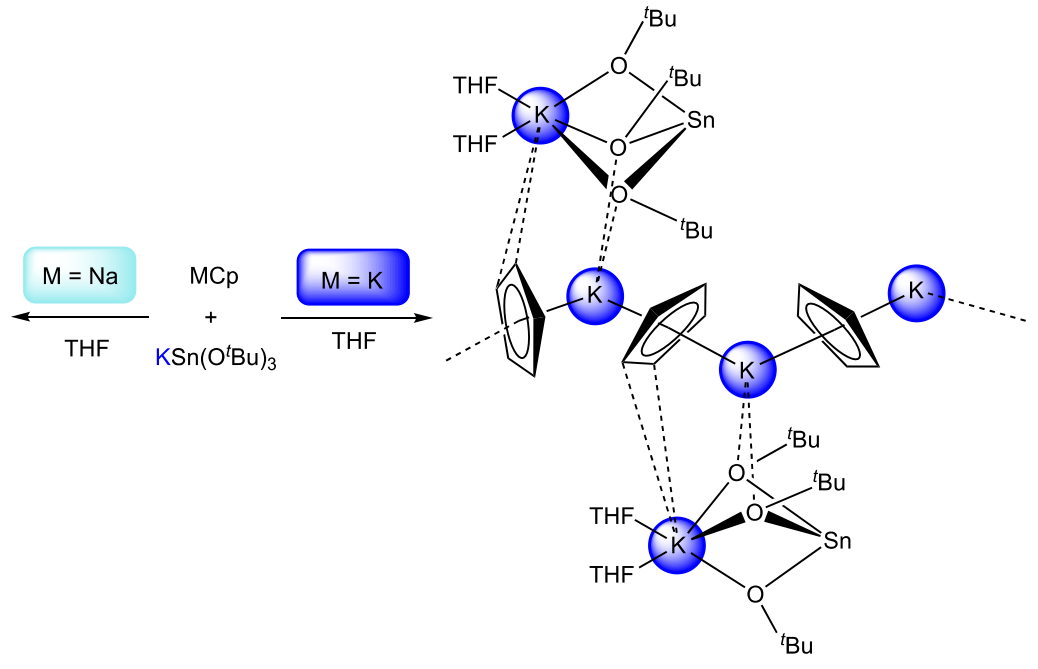

(10)

Though prepared in an aqueous medium, it is worth mentioning the supramolecular disodium tetrapotassium compound $\mathrm{Na}_{2} \mathrm{~K}_{4}$ [p-sulfonatothiacalix[4]arene] $\cdot 7 \mathrm{H}_{2} \mathrm{O}$ since it was made by a partial sodium-potassium exchange process promoted by adding potassium carbonate to $\mathrm{Na}_{4}\left[p\right.$-sulfonatothiacalix[4]arene]. ${ }^{41}$ This heterometallic structure is novel in that it represents the first example in calixarene or thiacalixarene chemistry demonstrating interactions between metal coordinated water molecules and aromatic $\pi$ electrons.

A combination of two different alkali metal bases, potassium hydride and butyllithium proved to be effective in deprotonating both $\mathrm{N}-\mathrm{H}$ bonds of 1,2bis(neopentylamino)benzene, whereas excess potassium hydride under the same conditions (solvent THF) only managed monodeprotonation (Scheme 14). ${ }^{42}$

Scheme 14. Contrasting deprotonative power of monometallic $\mathrm{KH}$ and the bimetallic $\mathrm{KH} /{ }^{\mathrm{B} B u L i}$ mixture. 

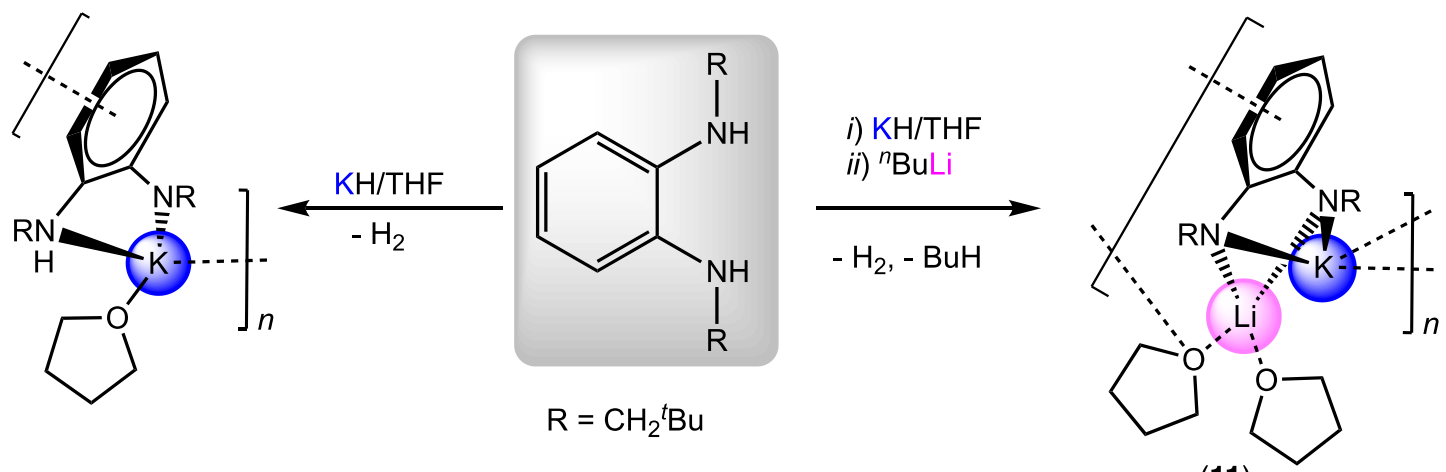

(11)

The heterobimetallic product $\left[(\mathrm{THF})_{2} \mathrm{LiK}\left\{\mathrm{C}_{6} \mathrm{H}_{4}-1,2-\left(\mathrm{N}-\mathrm{CH}_{2}{ }^{t} \mathrm{Bu}\right)_{2}\right\}\right]$ (11) adopts a polymeric strand structure propagated by $\mathrm{K}-\pi$ interactions involving the centroid of the side-on ligated phenylene unit.

The homo-alkali-metal samarium cluster complexes $\left[\mathrm{Sm}_{4} \mathrm{~K}_{2} \mathrm{O}\left(\mathrm{OCH}_{2} \mathrm{CH}_{2} \mathrm{NMe}_{2}\right)_{26}(\mathrm{OH})_{6}\right]$ (12), and $\left[\mathrm{Sm}_{2} \mathrm{Na}_{8}\left(\mathrm{OCH}_{2} \mathrm{CH}_{2} \mathrm{NMe}_{2}\right)_{12}(\mathrm{OH})_{2}\right](13)$, and the hetero-alkali-metallic analog $\left[\mathrm{Sm}_{2} \mathrm{~K}_{12} \mathrm{Na}_{4}\left(\mathrm{OCH}_{2} \mathrm{CH}_{2} \mathrm{NMe}_{2}\right)_{18}(\mathrm{OH})_{4}\right](\mathbf{1 4}$, Figure 11) can all act as single component initiators for ring-opening polymerization of the cyclic ester monomers $\varepsilon$-caprolactone $(\varepsilon-C L)$, L-lactide (L-LA) and trimethylene carbonate. ${ }^{43}$

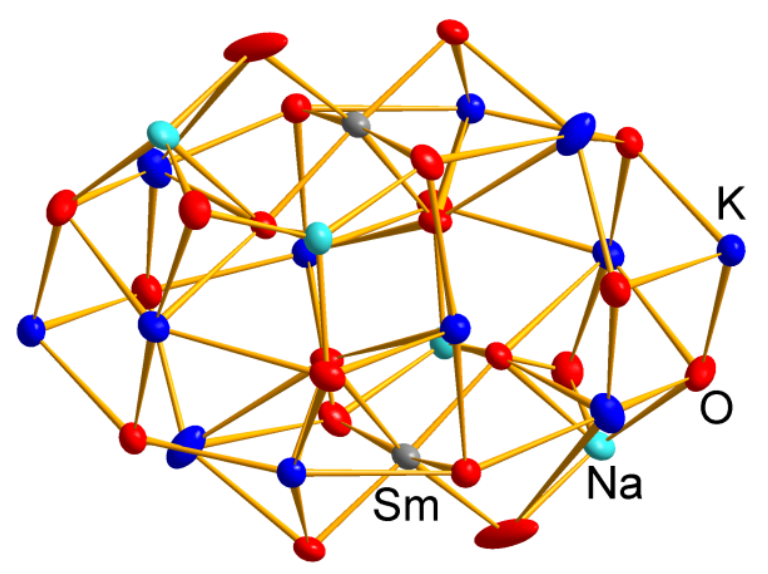

Figure 11. Core molecular structure of $\left[\mathrm{Sm}_{2} \mathrm{~K}_{12} \mathrm{Na}_{4}\left(\mathrm{OCH}_{2} \mathrm{CH}_{2} \mathrm{NMe}_{2}\right)_{18}(\mathrm{OH})_{4}\right](14)$.

An interesting sodium-potassium synergistic effect is observed when comparing 
activities as for example the hetero potassium-sodium cluster $\mathbf{1 4}$ could polymerize 15,000 equivalents of $\varepsilon-\mathrm{CL}$ in $1 \mathrm{~min}$ at $25^{\circ} \mathrm{C}$ and the polymer yield could still reach $66 \%$ when the monomer/initiator ratio increased to 20,000 ; whereas the homometallic potassium and sodium clusters $\mathbf{1 2}$ and $\mathbf{1 3}$ gave much lower activities with the desired results at $25^{\circ} \mathrm{C}$ only achieved when the molar ratio of monomer/initiator is decreased to 10,000 or 6,000 respectively. However, this increased activity also correlates with an increase in the alkali metal:samarium molar ratio.

The solvothermal organic conversion of the tertiary carbonitrile, 2,2diphenylpropanenitrile to 1,1-diphenylethane also displayed an interesting inorganic lithium-sodium synergistic effect (Scheme 15).

Scheme 15. A reductive decyanation reaction using a sodium hydride - alkali metal iodide protocol.<smiles>CC(C#N)(c1ccccc1)c1ccccc1</smiles>

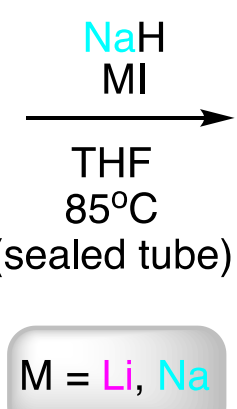<smiles>CC(c1ccccc1)c1ccccc1</smiles>

Three equivalents of $\mathrm{NaH}$ was not sufficient to effect reductive decyanation, though introducing two equivalents of Nal gave a 96\% yield of the product after 14 hours in a sealed tube in THF solution at $85^{\circ} \mathrm{C}$. However, the addition of one equivalent of Lil to three equivalents of $\mathrm{NaH}$ afforded a $98 \%$ yield of the product in a much more rapid 3.5 hours. ${ }^{44}$ Proof of the heterobimetallic synergistic effect was realised since the 
reduction did not occur at all when a LiH/Lil combination was used. The scope of this unique sodium hydride nucleophilic donor reactivity was increased to reduction reactions of a series of carbonitriles, as well as a small selection of amides and imines. The sodium hydride - lithium iodide partnership was extended to hydrodebromination and hydrodeiodination of halo(hetero)arenes. ${ }^{45}$ Mechanistic studies showed that the reactions occur via a concerted nucleophilic aromatic substitution pathway. Beyond reduction, this sodium hydride - lithium iodide protocol finds application in directed sodiation ( $\mathrm{Na}-\mathrm{H}$ exchange) reactions. ${ }^{46}$ This includes its role in converting $\alpha$-arylacetamides to 2 -indanones, compounds important in the manufacture of pharmaceuticals. In the mechanistic pathway, lateral-sodiation of the benzylic $\mathrm{sp}^{2}-\mathrm{C}-\mathrm{H}$ substituent occurs (Scheme 16).

Scheme 16. Mechanism of the conversion of $\alpha$-arylacetamides to 2 -indanones using a sodium hydride - lithium iodide protocol.
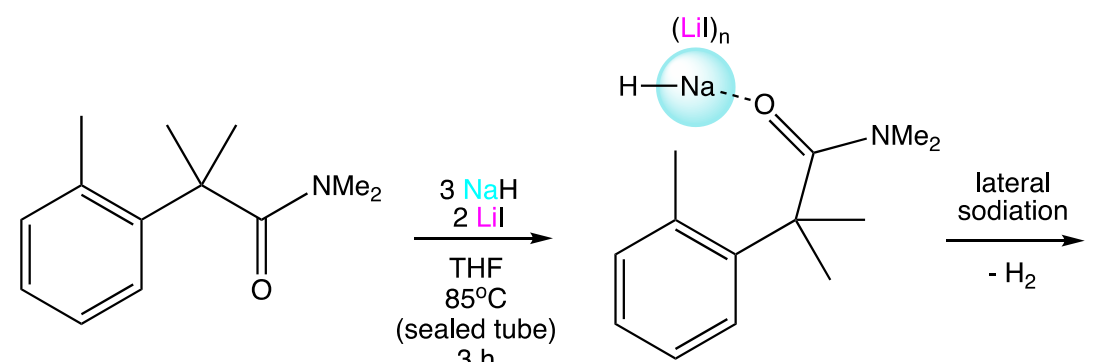<smiles>CNCc1ccccc1C(C)(C)C(=O)NC</smiles>

$3 \mathrm{~h}$
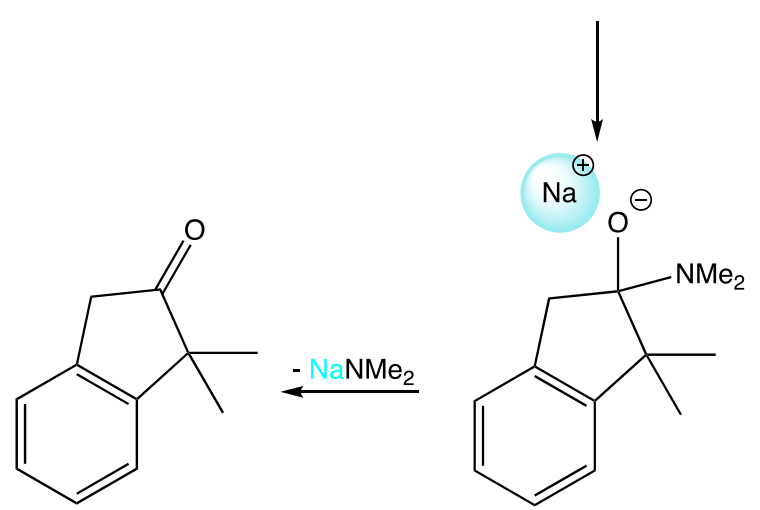
Other $\alpha$-arylacetamides without benzylic groups can undergo ortho-sodiation of $\mathrm{sp}^{2}$ $\mathrm{C}-\mathrm{H}$ bonds directed by the amide substituent. A mechanism has been postulated for this transformation (Scheme 17).

Scheme 17. Mechanism of amide reduction of an $\alpha$-arylacetamide and its conversion to an arylaldehyde derivative using an alkali metal hydride - iodide protocol.<smiles>CC(C)c1cc2ccccc2cc1C=O</smiles>

Following ortho deprotonation/sodiation, nucleophilic addition to the amide carbonyl furnishes a four-membered ring anionic carbinolamine, which then undergoes ring opening via $\mathrm{C}-\mathrm{C}$ bond cleavage to generate an arylamide. Finally, hydride reduction of the amide group leads to the formation of the aldehyde product. The utility of this sodium hydride - lithium iodide partnership has also been applied successfully to nucleophilic amination reactions of methoxyarenes that provide access to benzannulated saturated nitrogen heterocycles. ${ }^{47}$ The reaction is thought to take place through a concerted nucleophilic aromatic substitution pathway (Scheme 18).

Scheme 18. Synthesis of benzannulated saturated nitrogen heterocycles from methoxy-aryl precursors. 
<smiles></smiles>

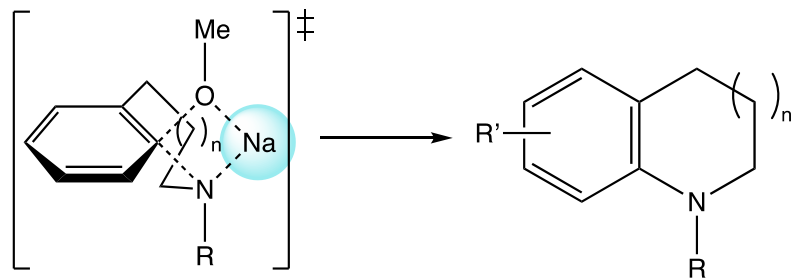

Arylphosphine oxides have also been dearylated by 2:1 stoichiometric mixtures of sodium hydride and lithium iodide in THF solution at $60{ }^{\circ} \mathrm{C}$ (Scheme 19).

Scheme 19. Hydrodearylation of triarylphosphine oxides using the sodium hydride lithium iodide protocol.

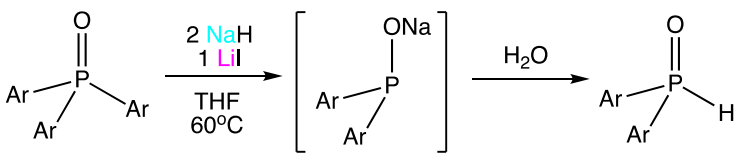

\begin{tabular}{|l|l|l|}
\hline & time (h) & yield (\%) \\
\hline
\end{tabular}

Optimized through triphenylphosphine oxide, following hydrolysis of the transient sodium phosphinite the yield of diphenylphosphine was 98\%; whereas in the absence of lithium iodide the yield fell to $2 \%{ }^{48}$ The method proved compatible with both electron-rich and bulky triarylphosphine oxides. Transient sodium phosphinites could 
be successfully intercepted with various electrophiles including primary and secondary alkyl halides, aldehydes and imines. Unsymmetrical tertiary phosphine oxides could also be made using this approach. A study using a battery of experimental techniques including powder X-ray diffraction, solid-state NMR and X-ray photoelectron spectroscopies supported by DFT calculations has probed the origins of the special hydride reactivity of the NaH-Nal and NaH-Lil partnerships. ${ }^{49}$ It is found that mixtures of $\mathrm{NaH}$ and $\mathrm{Nal}$ or $\mathrm{NaH}$ and Lil in THF solvent provide new inorganic composites. On studying these composites the authors conclude "synergistic cooperation between $\mathrm{NaH}$ and $\mathrm{Nal}$ at the surface of the composite is proposed to be critical for the observed hydride-donor chemical reactivity to $\mathrm{NaH}^{\prime \prime}$. Other notable findings include the absence of any Lil structural units in the heterobimetallic $\mathrm{NaH}-\mathrm{Lil}$ composite, though Li appears to be present in the form of LiH via solvothermal salt metathesis.

\subsection{Group 2 Chemistry}

\subsubsection{Inverse Crown and Related Complexes}

Organomagnesium chemistry is clearly an area embossed with a strong synergistic stamp. Alkali metal mediation is particularly evident in "inverse crown" chemistry, since informative examples well defined both in terms of reactivity and structural characterisation are plentiful. Inverse crown complexes are constructed from cationic rings made up of alternating metal and nitrogen centers, the cores of which are occupied by charge-balancing anions. The first examples were labelled inverse crown "ethers" as they contained oxide cores, for example in the mixed lithium-magnesium amide $\left[\mathrm{Li}_{2} \mathrm{Mg}_{2}(\mathrm{TMP})_{4}(\mathrm{O})\right](\mathbf{1 5})$, and since their structures display Lewis acidic host rings 
with Lewis basic host guests they were considered the inverse of conventional crown ether complexes, which have oxygen atoms in host rings and metal atoms in the core. The ether label was dropped when new types with more complex anionic arene cores started to emerge. ${ }^{50-52}$ In these types arenes such as benzene or toluene were doubly deprotonated by the mixed alkyl-amido complex "[NaMg$\left.(\mathrm{TMP})_{2}\left({ }^{n} \mathrm{Bu}\right)\right]$ " (16) to produce isolable crystalline inverse crowns of formula [ $\mathrm{Na}_{4} \mathrm{Mg}_{2}(\mathrm{TMP})_{6}($ arene-2H)] (scheme $20 ; \mathrm{R}=\mathrm{H}, \mathbf{1 7} ; \mathrm{Me}, \mathbf{1 8}$ ), where arene-2H represent a doubly deprotonated parent arene. To reflect their synergistic nature such reactions were labelled alkalimetal-mediated magnesiations (AMMMg), since without sodium mediation the magnesium components of 16 , namely $\mathrm{Mg}(\mathrm{TMP})_{2}$ and $\mathrm{Bu}_{2} \mathrm{Mg}$ cannot deprotonate benzene.

Scheme 20. Formation of inverse crowns containing arenediide cores, with the two deprotonated sites occupied by Mg centers.

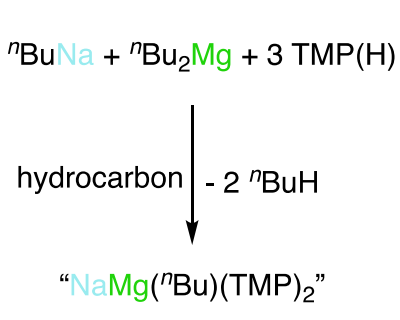

(16)
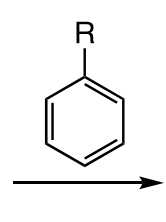

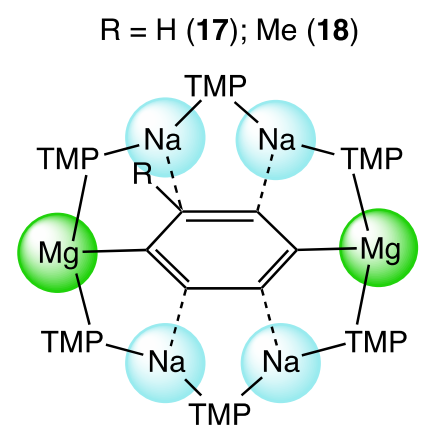

In 2014 the first pre-inverse crowns were characterized. These reagents are defined as the alkali metal magnesiate species that exist pre-metalation, which carry out the synergistic deprotonations. A key example is crystallographically characterized $\left[\mathrm{KMg}(\mathrm{TMP})_{2}{ }^{n} \mathrm{Bu}\right]_{6}(19)$, a hexamer displaying a 24 -membered $(\mathrm{KNMgN})_{6}$ ring, with its 
endo-oriented $n$-butyl ligands bound to magnesium. DOSY experiments confirmed retention of its hexameric state in cyclo- $C_{6} D_{12}$ solution. Reaction with naphthalene in methylcyclohexane/heptane solution produced the inverse crown $\left[\mathrm{KMg}(\mathrm{TMP})_{2}(2-\right.$ $\left.\left.\mathrm{C}_{10} \mathrm{H}_{7}\right)\right]_{6}(20)$, with the six ${ }^{n} \mathrm{Bu}$ ligands being replaced by six naphthalene molecules deprotonated regioselectively at position C2 (Figure 12). ${ }^{53}$
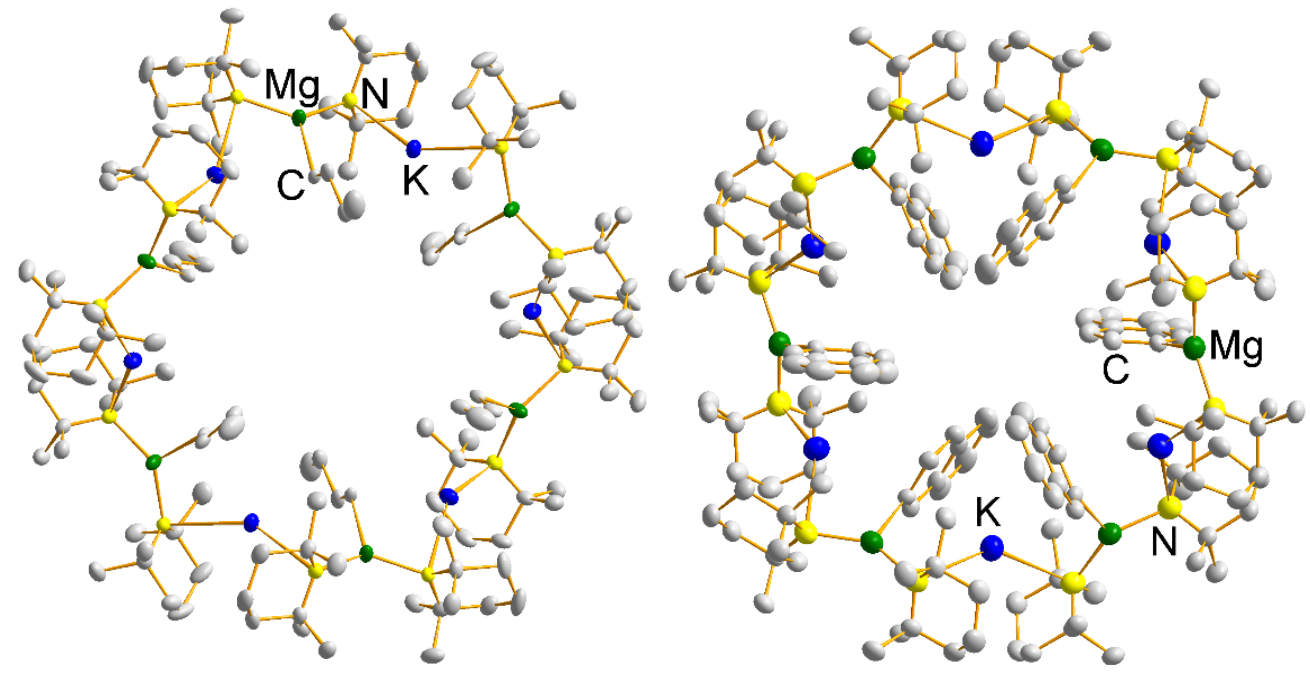

Figure 12. Reaction of naphthalene with the pre-inverse crown $\left[\mathrm{KMg}(\mathrm{TMP}){ }_{2}{ }^{n} \mathrm{Bu}\right]_{6}(\mathbf{1 9}$, LHS) forms the inverse crown $\left[\mathrm{KMg}(\mathrm{TMP})_{2}\left(2-\mathrm{C}_{10} \mathrm{H}_{7}\right)\right]_{6}(20, \mathrm{RHS})$, which retains the 24membered $(\mathrm{KNMgN})_{6}$ ring.

Thus it was concluded that the reaction was template controlled. In contrast, switching to the aforementioned pre-inverse-crown "[NaMg(TMP $\left.)_{2}{ }^{n} \mathrm{Bu}\right]$ " 16 produced an unprecedented 1,4-regioselective dimagnesiation of naphthalene in $\left[\left\{\mathrm{Na}_{4} \mathrm{Mg}_{2}(\mathrm{TMP})_{4}\left(\mathrm{TMP}^{*}\right)_{2}\right\}\left(1,4-\mathrm{C}_{10} \mathrm{H}_{6}\right)\right] \quad$ (where $\mathrm{TMP}^{*} \quad$ is $2,2,6$-trimethyl-1,2,3,4tetrahydropyridide, a demethylated variant of TMP, 21, Figure 13). 


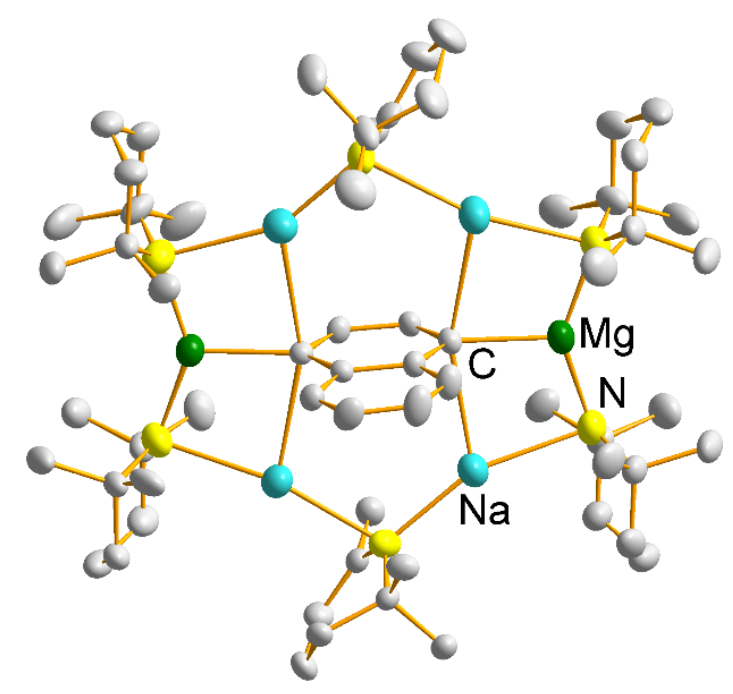

Figure 13. Molecular structure of the inverse crown $\left[\left\{\mathrm{Na}_{4} \mathrm{Mg}_{2}(\mathrm{TMP})_{4}\left(\mathrm{TMP}^{*}\right)_{2}\right\}(1,4-\right.$ $\left.\mathrm{C}_{10} \mathrm{H}_{6}\right)$ ] 21, where TMP* is 2,2,6-trimethyl-1,2,3,4-tetrahydropyridide.

The implication that the template for this reaction was a smaller 12 -membered preinverse-crown of formula $\left[\mathrm{Na}_{4} \mathrm{Mg}_{2}(\mathrm{TMP})_{6}\left({ }^{n} \mathrm{Bu}\right)_{2}\right]\left(\mathbf{1 6}^{\prime}\right)$ was proven indirectly (no crystallographic evidence is known) by subjecting a series of functionalized arene substrates to a 2:1:3 stoichiometric mixture of ${ }^{n} \mathrm{BuNa},{ }^{n} \mathrm{Bu}_{2} \mathrm{Mg}$, and $\mathrm{TMP}(\mathrm{H})$ in methylcyclohexane solution. ${ }^{54}$ Without exception all substrates studied gave doubly deprotonated dimagnesiated intermediates where the magnesium occupies either ortho, meta' (e.g., with anisole, 22; Figure 14, left) or meta, meta' positions (e.g., with $\mathrm{N}, \mathrm{N}$-dimethylaniline, 23; Figure 14, right) in high yields. 

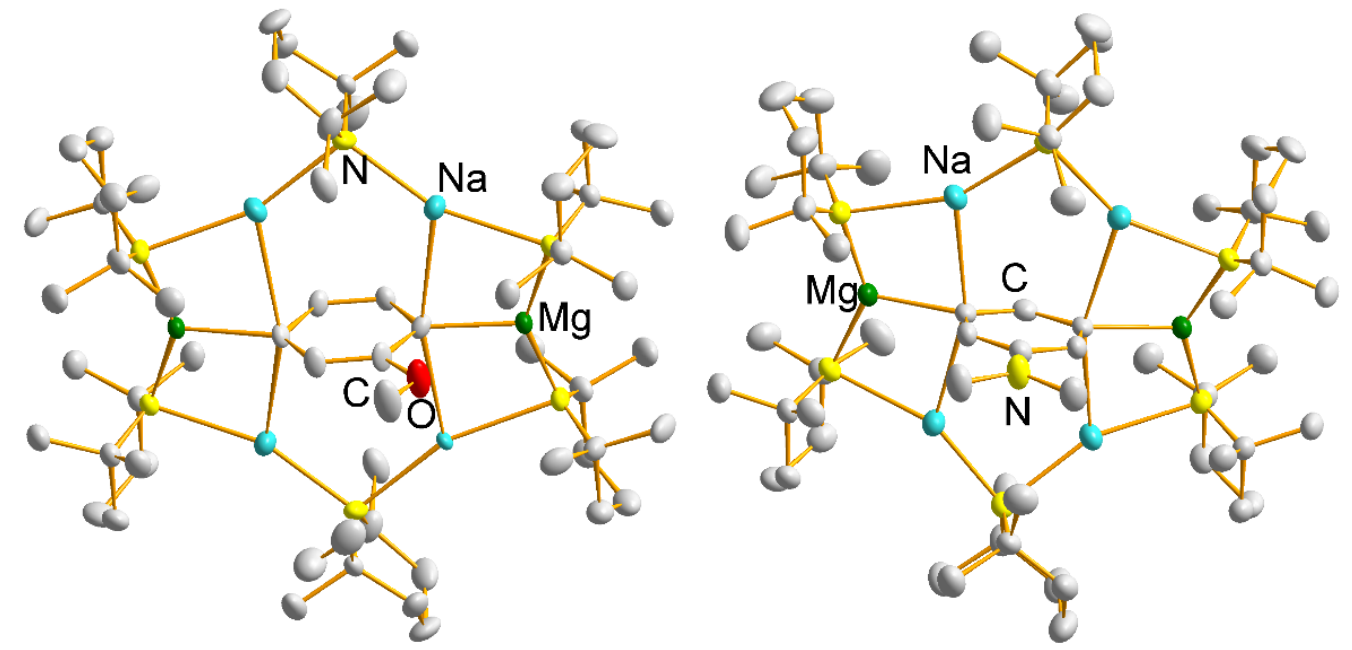

Figure 14. Inverse crowns with ortho, meta' dideprotonated anisole (22, LHS) and meta, meta' dideprotonated $\mathrm{N}, \mathrm{N}$-dimethylaniline $(\mathbf{2 3}, \mathrm{RHS})$ cores.

The full list of reactions showing the tri-substituted arene products following reaction with electrophiles (Scheme 21), covers substrates with substituents that generally direct metalation to ortho positions.

Scheme 21. A selection of dideprotonation reactions of monosubstituted benzenes using the sodium-magnesium template base $\left[\mathrm{Na}_{4} \mathrm{Mg}_{2}(\mathrm{TMP})_{6}\left({ }^{n} \mathrm{Bu}\right)_{2}\right] \mathbf{1 6}^{\prime}$. 


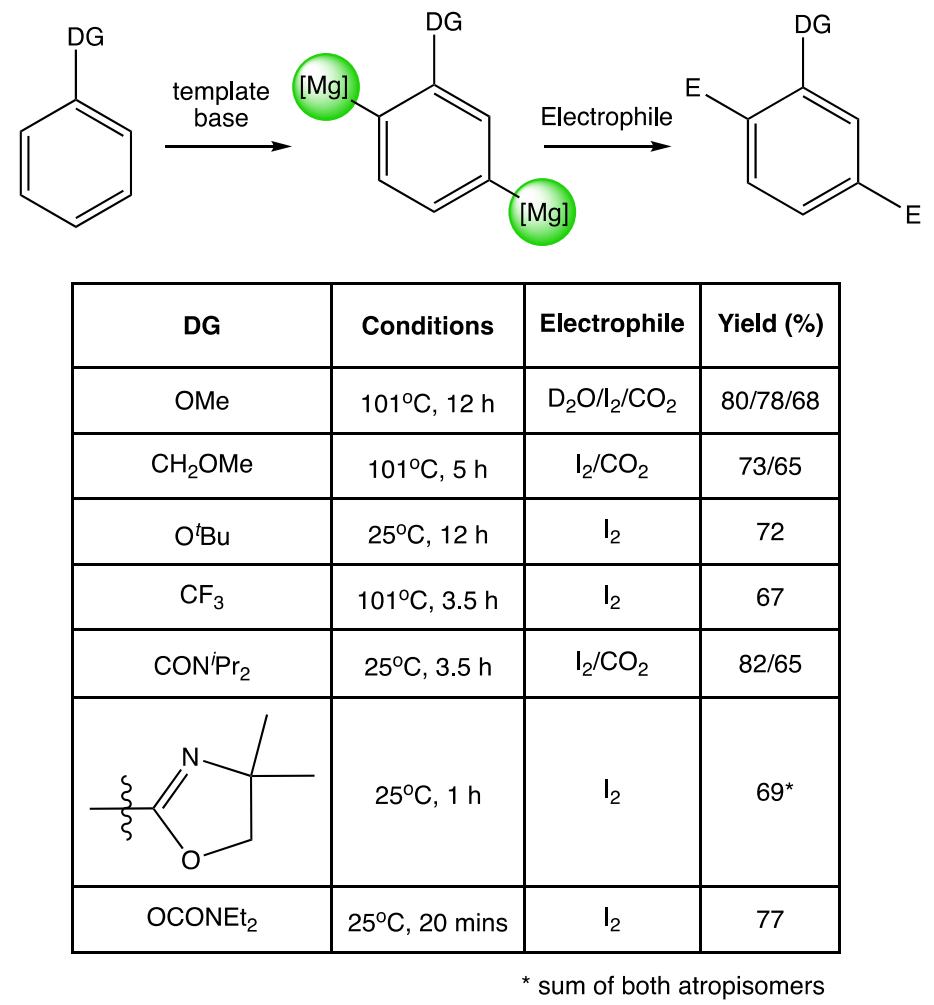

Directed ortho-metalation (DoM) is the seminal concept in organolithium chemistry, ${ }^{55-}$

58 which is governed by the electronic and/or coordinative characteristics of a directing group attached to the benzene ring. In perhaps the most important demonstration of the power of alkali metal mediation to date, here in these examples DoM is overridden by the template structure of the mixed-metal base, where the order of and regioselectivity of metalation is commensurate with the number and position of $n$-butyl ligands in the template ring.

Inverse crown chemistry and template metalation has been extended to polyaryl substrates, the functionalization of which yields molecules of interest to an assortment of industries (for example, dealing with molecular electronics, smart materials, or pharmaceuticals). Unactivated polyaryls, in the sense that they lack substituents other than hydrogen, have been investigated in company with the aforementioned $\left[\mathrm{NaMg}(\mathrm{TMP}) 2_{2}{ }^{n} \mathrm{Bu}\right] \quad$ (16). ${ }^{59}$ For example, para-terphenyl has undergone a double deprotonation reaction on one terminal $\mathrm{Ph}$ ring to convert to the 
mono-inverse-crown product, $\left[\mathrm{Na}_{4} \mathrm{Mg}_{2}(\mathrm{TMP})_{6}(3,5\right.$-para-terphenyl-di-ide)] (24), or four times (twofold on each terminal Ph ring) to produce the bis-inverse-crown analog $\left[\left\{\mathrm{Na}_{4} \mathrm{Mg}_{2}(\mathrm{TMP})_{6}\right\}_{2}\left(3,3^{\prime}, 5,5^{\prime}\right.\right.$-para-terphenyl-tetra-ide) $](\mathbf{2 5}$, scheme 22$) .{ }^{60}$

Scheme 22. Formation of $\left[\mathrm{Na}_{4} \mathrm{Mg}_{2}(\mathrm{TMP})_{6}\right.$ (3,5-para-terphenyl-di-ide) $]$ (24, LHS) and $\left[\left\{\mathrm{Na}_{4} \mathrm{Mg}_{2}(\mathrm{TMP})_{6}\right\}_{2}\left(3,3^{\prime}, 5,5^{\prime}\right.\right.$-para-terphenyl-tetra-ide) $](\mathbf{2 5}, \mathrm{RHS})$ using the template base $\left[\mathrm{NaMg}(\mathrm{TMP}) 2^{n} \mathrm{Bu}\right]$ in methylcyclohexane solution.

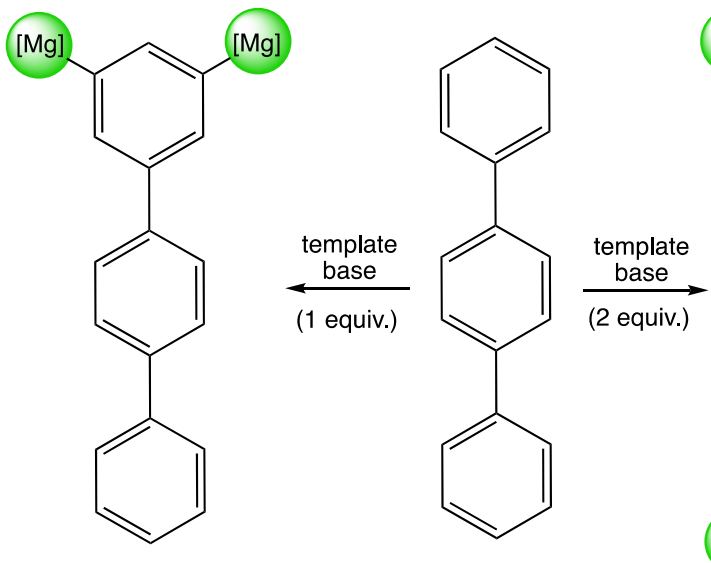

(24)

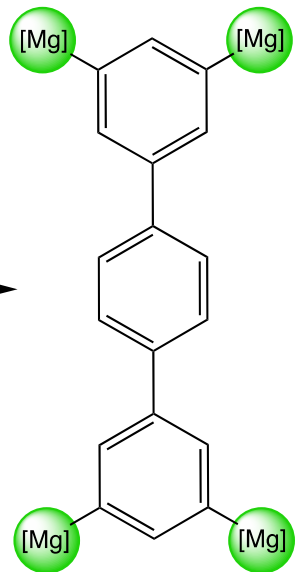

(25)

This latter complex established that it was possible to perform template metalation with stoichiometric control (two equivalents of base gave this product; whereas one produced dimetalation) and incorporate two inverse crowns within a single molecular structure. An earlier example of fourfold deprotonation was reported for the monoinverse-crown derived from ferrocene, namely $\left[\mathrm{Na}_{4} \mathrm{Mg}_{4}\left(\mathrm{~N}^{i} \mathrm{Pr}_{2}\right)_{8}\left\{\mathrm{Fe}\left(\mathrm{C}_{5} \mathrm{H}_{3}\right)_{2}\right\}\right], \quad 26 .{ }^{61}$ Interestingly, the anti-aromatic substrate biphenylene was selectively dimagnesiated at the 1,4-positions using 16 . Thus far, the clear pattern emerging from such sodiummediated template metalation is that depending on the substrate twofold metalation takes place regioselectively at either 3,5-positions in arenes (or 1,3 in ferrocene), where the C-Mg bonds are separated by one $\mathrm{C}-\mathrm{H}$ bond or at the 2,5-positions, where 
the $\mathrm{C}-\mathrm{Mg}$ bonds are separated by two $\mathrm{C}-\mathrm{H}$ bonds. Currently, template metalation offers applicability in synthetic campaigns of useful materials where twofold metalation is required in these positions for onward functionalisation. Template metalation of biphenyl to the inverse crown $\left[\mathrm{Na}_{4} \mathrm{Mg}_{2}(\mathrm{TMP})_{6}(3,5\right.$-biphenyl-di-ide)] (27), and subsequent iodination and copper-catalyzed Ullmann-type coupling of carbazole generates 3,5-bis( $N$-carbazolyl)-1,1'-biphenyl (Scheme 23$)$, which is closely related to organic light-emitting diode materials.

Scheme 23. Synthesis of 3,5-bis( $N$-carbazolyl)-1,1'-biphenyl using [ $\left.\mathrm{NaMg}(\mathrm{TMP})_{2}{ }^{n} \mathrm{Bu}\right]$ 16 as a template base.

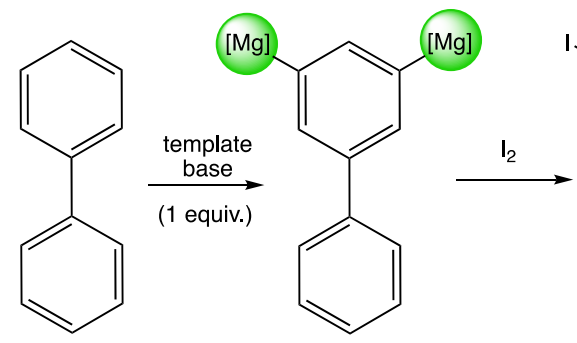

(27)

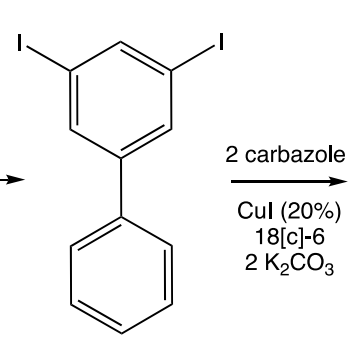

$\underset{\mathrm{Cul}(20 \%)}{\mathrm{carbazole}}$
$18[\mathrm{c}]-6$
$2 \mathrm{~K}_{2} \mathrm{CO}_{3}$<smiles>c1ccc(-c2cc(-n3c4ccccc4c4ccccc43)cc(-n3c4ccccc4c4ccccc43)c2)cc1</smiles>

\subsubsection{Magnesiates Involving Trimethylsilylmethyl and Related Ligands}

A series of well-defined potassium magnesiates based on the bulky trimethylsilylmethyl ligand $\mathrm{R}\left(=\mathrm{CH}_{2} \mathrm{SiMe}_{3}\right)$ have been prepared via cocomplexation (mixing of monometallic components). Important among them is potassium magnesiate $\left[(\mathrm{PMDETA})_{2} \mathrm{~K}_{2} \mathrm{MgR}_{4}\right]$ (28). Benchmarking deprotonation reactions with anisole unveiled an emphatic synergistic effect with this higher order (2:1, K:Mg ratio) magnesiate significantly outperforming its monometallic components $\mathrm{KR}$ and $\mathrm{MgR}_{2}$, its lower order (1:1, $\mathrm{K}: \mathrm{Mg}$ ratio) analog $\mathrm{KMgR}_{3}(29)$ as well as lighter lithium and sodium congeners $\left(\mathrm{M}_{2} \mathrm{MgR}_{4} ; \mathrm{M}=\mathrm{Li}, \mathbf{3 0} ; \mathrm{Na}, \mathbf{3 1}\right)$ (Scheme 24$) .{ }^{62}$ 
Scheme 24. Comparison of the efficiency of a series of $\mathrm{Me}_{3} \mathrm{SiCH}_{2}$ containing bases in benchmarking anisole deprotonation reactions.<smiles>COc1ccccc1</smiles>

\begin{tabular}{|c|c|}
\hline MR & $\begin{array}{c}\text { Conversion } \\
(\%)\end{array}$ \\
\hline $\mathrm{MgR}_{2}$ & 0 \\
\hline $\mathrm{KR}$ & 20 \\
\hline $\mathrm{KMgR}_{3}(\mathbf{2 9})$ & 15 \\
\hline$(\mathrm{PMDETA})_{2} \mathrm{~K}_{2} \mathrm{MgR}_{4}(\mathbf{2 8})$ & 99 \\
\hline$(\mathrm{TMEDA})_{2} \mathrm{Li}_{2} \mathrm{MgR}_{4}(\mathbf{3 0})$ & 5 \\
\hline$(\mathrm{TMEDA})_{2} \mathrm{Na}_{2} \mathrm{MgR}_{4}(\mathbf{3 1})$ & 10 \\
\hline $\begin{array}{c}(\mathrm{PMDETA})_{2} \mathrm{~K}_{2} \mathrm{MgR}_{4}(\mathbf{2 8}) \\
+18[\mathrm{C}]-6\end{array}$ & 0 \\
\hline
\end{tabular}

Magnesiate 28 proved an exceptionally effective reagent for the regioselective deprotonation of a wide range of aromatic and heteroaromatic substrates. Since its reactivity shutdown in the presence of the $\mathrm{K}^{+}$cation loving 18-crown-6 macrocyclic ligand, it is likely that these magnesium-hydrogen exchange reactions are genuine examples of potassium-mediated magnesiations operating via contacted-ion pair (CIP) mixed-metal intermediates, akin to the structure of $\left[(\mathrm{PMDETA})_{2} \mathrm{~K}_{2} \mathrm{MgR} \mathrm{R}_{4}\right]$ in the crystal (Figure 15).

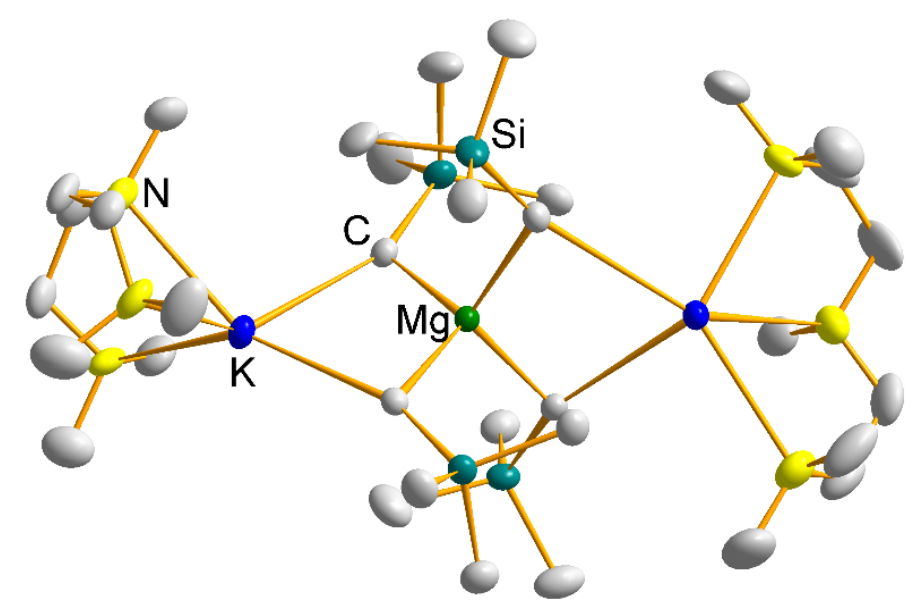

Figure 15. Molecular structure of crystalline [(PMDETA $\left.)_{2} \mathrm{~K}_{2} \mathrm{MgR}_{4}\right]$ (28). 
The homoleptic trimethylsilylmethyl-ligated sodium magnesiate $\left[\mathrm{NaMg}\left(\mathrm{CH}_{2} \mathrm{SiMe}_{3}\right)_{3}\right]$ (29) functions as a synergistic precatalyst for selective promotion of the hydroamination/trimerization of isocyanates (RNCO). ${ }^{63} \mathrm{~A}$ variety of arylamines (diphenyl, di-p-tolyl, $N$-methylaniline) and isocyanates ( $\left.\mathrm{R}={ }^{t} \mathrm{Bu}, \mathrm{Cy}, \mathrm{Ad}\right)$ using $2 \mathrm{~mol} \%$ of the precatalyst in $\mathrm{C}_{6} \mathrm{D}_{6}$ engaged in straightforward hydroamination to generate the relevant urea in high yield exceeding $90 \%$ (Scheme 25$)$.

Scheme 25. Catalytic hydroamination of isocyanates.

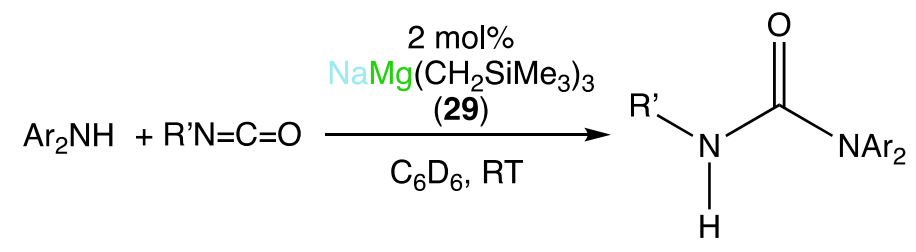

Reactions with 2,2-dipyridylamine gave poorer yields (11-58\%), while $\left(\mathrm{Me}_{3} \mathrm{Si}\right)_{2} \mathrm{NH}$ failed completely. Since 29 deprotonated three molar equivalents of $\mathrm{Ph}_{2} \mathrm{NH}$ in hexane-THF solution to produce $\left[(\mathrm{THF}) \mathrm{NaMg}\left(\mathrm{NPh}_{2}\right)_{3}(\mathrm{THF})\right](30)$, this implied a reaction of this type was the opening step in the mechanism of urea formation, with all three amido groups then undergoing insertion to form tris(ureido) species. Evidence for such tris(ureido) species came from isolation of $\left[(\mathrm{THF})_{3} \mathrm{NaMg}\left\{\left({ }^{t} \mathrm{BuN}\right) \mathrm{C}\left(\mathrm{NPh}_{2}\right)(=\mathrm{O})\right\}_{3}\right]$ (31). Subjecting $\mathrm{Ph}_{2} \mathrm{NH}$ to the small sterically demanding and strongly electrophilic isocyanate pTolNCO did not follow this hydroamination cycle but instead produced the isocyanate trimerization product isocyanurate. These competing catalytic cycles are shown in Scheme 26.

Scheme 26. Competing catalytic cycles for forming urea and isocyanurate products. 


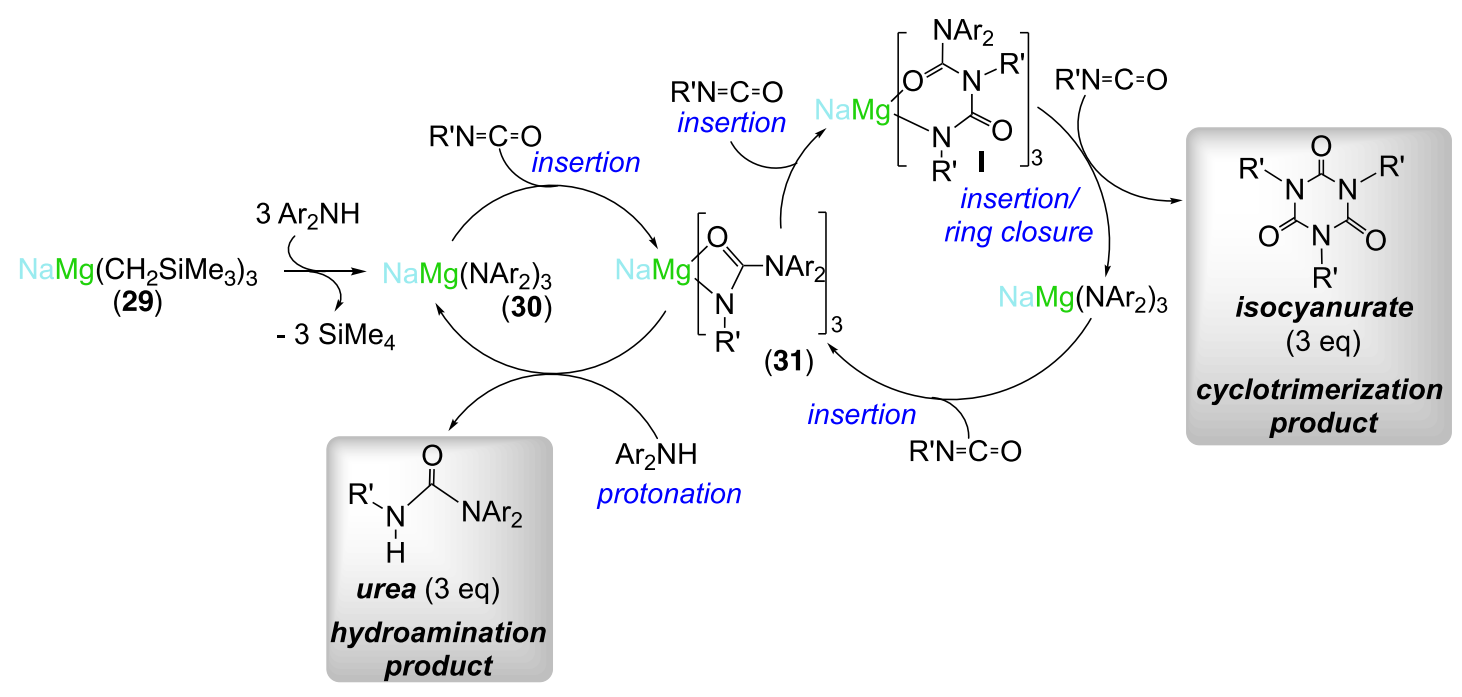

Sodium magnesiate $\mathbf{2 9}$ has also been shown to be an effective guanylation precatalyst in reactions of carbodiimides with different anilines and secondary amines (Scheme 27).

Scheme 27. Catalytic guanylation of carbodiimides using primary (RHS) and secondary amines (LHS).

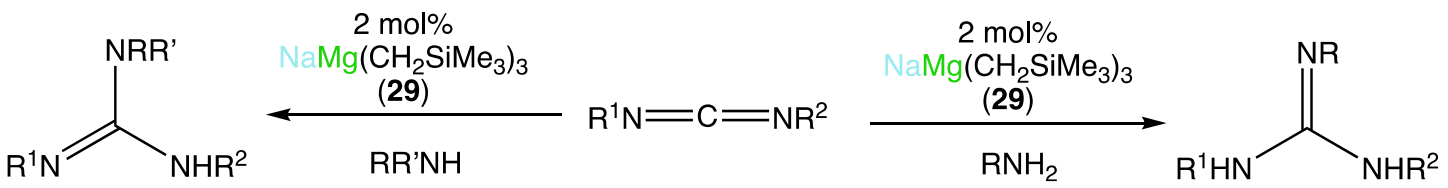

Again the poorer performance of the homometallic components $\left[\mathrm{NaCH}_{2} \mathrm{SiMe}_{3}\right]$ and [Mg( $\left.\left.\mathrm{CH}_{2} \mathrm{SiMe}_{3}\right)_{2}\right]\left(72 \%\right.$ and $44 \%$ respectively of product in $\mathrm{D}_{8}$ - THF solution after $0.25 \mathrm{~h}$; c.f., $99 \%$ for the magnesiate complex in the reaction of 2,6-dimethylaniline with ${ }^{i} \mathrm{PrN}=\mathrm{C}=\mathrm{N}^{i} \mathrm{Pr}$ using $2 \mathrm{~mol} \%$ of catalyst) signifies a significant synergistic effect. An extensive investigation looking at stoichiometric versions of these magnesiatecatalyzed reactions, molecular structures determined crystallographically and kinetic studies using ${ }^{1} \mathrm{H}$ NMR spectroscopic measurements allowed a mechanism to be 
postulated (scheme 28) and concluded the existence of an amine-assisted ratedetermining carbodiimide insertion transition state. In the same study catalytic hydrophosphination was also achieved with this magnesiate in reactions of diphenylphosphine with three different carbodiimides. ${ }^{64}$

Scheme 28. Postulated catalytic cycle for guanylation of carbodiimides using a sodium magnesiate catalyst (29).

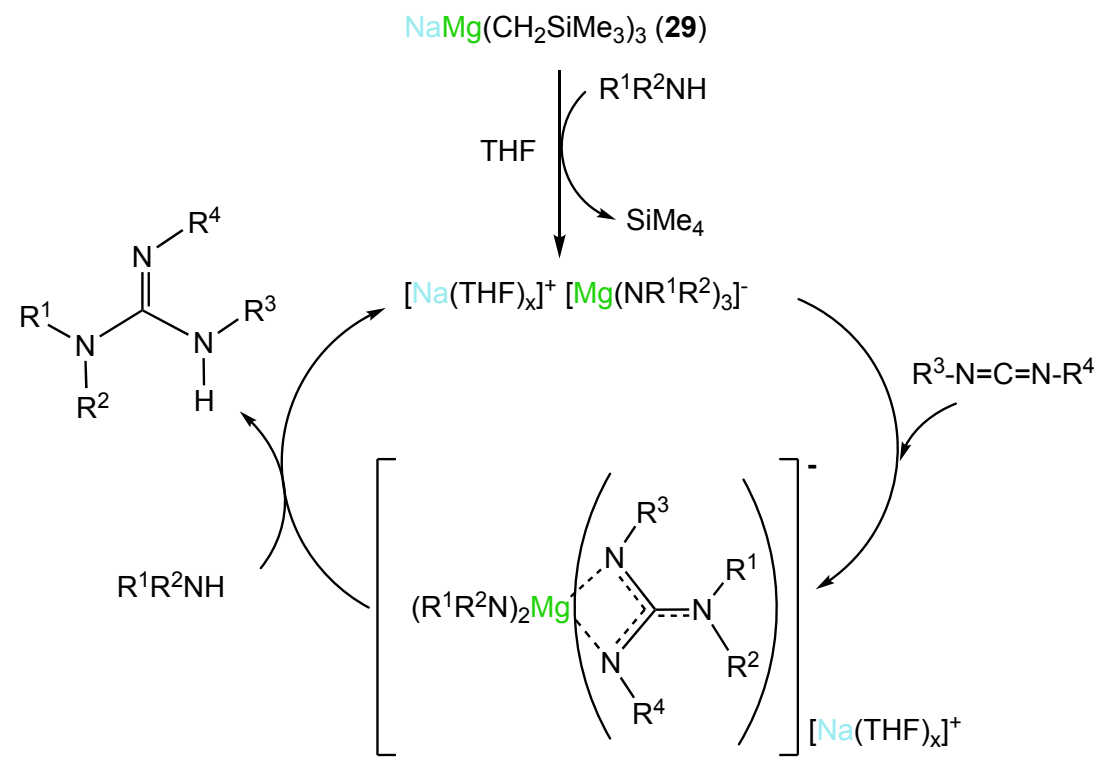

All of the aforementioned polar organometallic compounds are sensitive to air and moisture forming thermodynamically favorable metal-oxygen bonds. That said, in certain cases, controlled exposure to oxygen can lead to well-defined oxygen insertion products. For example, the homoleptic monomeric complex [(PMDETA) LiMg $\left.\left(\mathrm{CH}_{2} \mathrm{SiMe}_{3}\right)_{3}\right](32$, Scheme 29) could be transformed to the remarkable charge-separated species $\left[\left\{(\mathrm{PMDETA})_{2} \mathrm{Li}_{2} \mathrm{Mg}\left(\mathrm{CH}_{2} \mathrm{SiMe}_{3}\right)_{3}\right\}^{+}\right.$ $\left.\left\{\mathrm{Mg}_{3}\left(\mathrm{CH}_{2} \mathrm{SiMe}_{3}\right)_{6}\left(\mathrm{OCH}_{2} \mathrm{SiMe}_{3}\right)\right\}^{-}\right]$(33) on deliberate exposure to oxygen for 30 minutes (using a drying tube to avoid moisture contamination) in a respectable $34 \%$ yield. ${ }^{65}$ 
Scheme 29. Partial oxygenation of the lithium magnesiate complex [(PMDETA)LiMg $\left.\left(\mathrm{CH}_{2} \mathrm{SiMe}_{3}\right)_{3}\right], \mathbf{3 2}$.

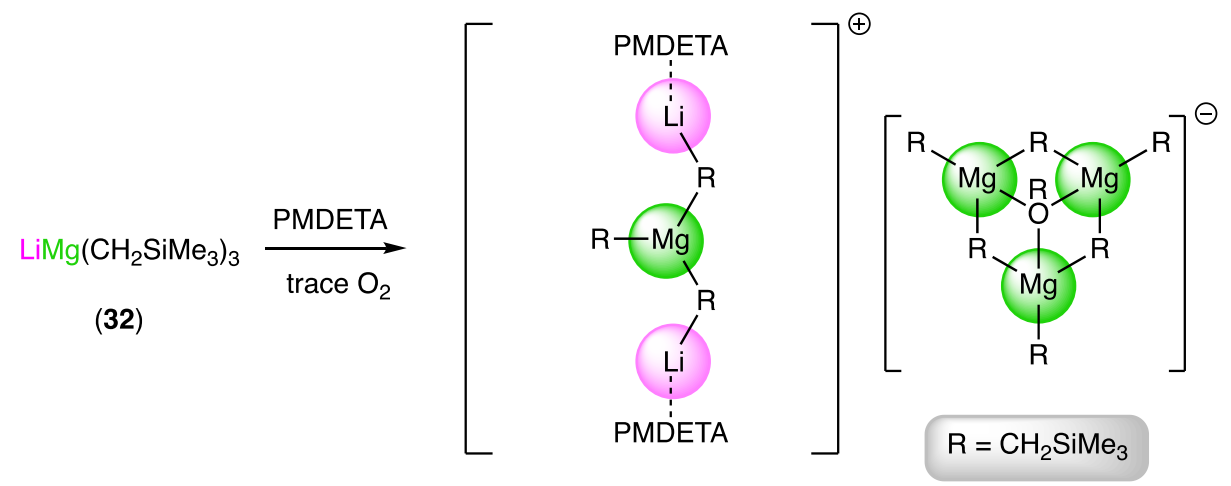

(33)

The partial oxygen insertion occurs only in one $\mathrm{Mg}-\mathrm{CH}_{2} \mathrm{SiMe}_{3}$ bond of the trinuclear anionic moiety. In the crystal, the $\mathrm{Mg}$ centers in the anion link via three $\mu_{2}$-alkyl ligands and one $\mu_{3}$-alkoxide $\mathrm{OCH}_{2} \mathrm{SiMe}_{3}$ ligand, with their coordination completed by three terminal alkyl groups, making overall a pseudo-cubane arrangement with a missing vertex (Figure 16). The cationic moiety represents a rare example of a cationic lithium magnesiate. It has a trialkyl-coordinated $\left(\mathrm{MgR}_{3}\right)^{-}$core, terminally bound at two ends by a PMDETA-solvated $\mathrm{Li}^{+}$cation.
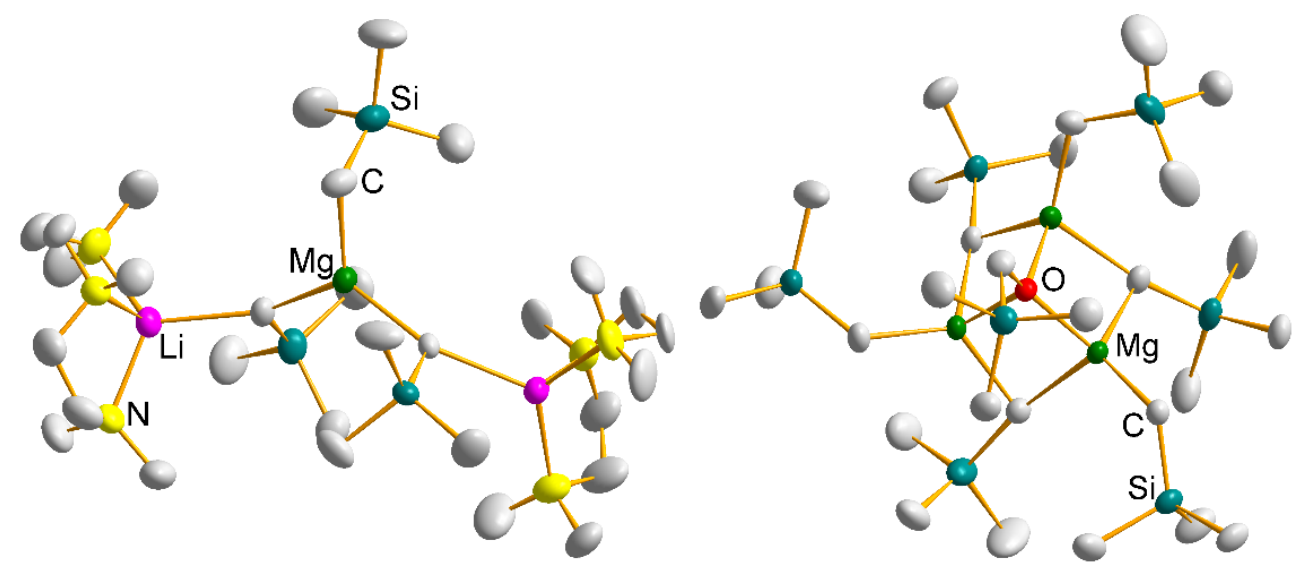

Figure 16. Molecular structure of the charge-separated ion pair complex $\left[\left\{(\mathrm{PMDETA})_{2} \mathrm{Li}_{2} \mathrm{Mg}\left(\mathrm{CH}_{2} \mathrm{SiMe}_{3}\right)_{3}\right\}^{+}\left\{\mathrm{Mg}_{3}\left(\mathrm{CH}_{2} \mathrm{SiMe}_{3}\right)_{6}\left(\mathrm{OCH}_{2} \mathrm{SiMe}_{3}\right)\right\}^{-}, \mathbf{3 3}\right.$. 
The

heteroleptic

sodium

butylmagnesiate

reagent

$\left[\left\{\mathrm{Na}(\mathrm{THF})_{6}\right\}^{+}\left\{\left(\mathrm{Ph}_{2} \mathrm{Si}\left(\mathrm{NAr}^{*}\right)_{2}\right) \mathrm{Mg}(\mathrm{Bu})(\mathrm{THF})\right\}^{-}\right]$(34) containing a dianionic chelating silyl(bisamido) ligand (where $A r^{*}=2,6-{ }^{-} \mathrm{Pr}_{2}-\mathrm{C}_{6} \mathrm{H}_{3}$ ) has proved an effective $\mathrm{AMMMg}$ reagent towards $N$-methylbenzimidazole $\left(\mathrm{blm}^{\mathrm{Me}}\right)$, pyrrole and 2,6-diisopropylaniline $\left(\mathrm{NH}_{2} \mathrm{Ar}{ }^{*}\right) . \mathrm{C} 2$ magnesiation occurs with blm ${ }^{\mathrm{Me}}$ manifest in a solvent-separated ion pair (SSIP) structure with a novel dinuclear dianion comprising two [( $\left.\mathrm{Ph}_{2} \mathrm{Si}(\mathrm{NAr} *)_{2}\right) \mathrm{Mg}$ ] fragments bridged by two $\mathrm{N}$-methylbenzimidazolyl blm ${ }^{\mathrm{Me}}$ ligands (35, Scheme 30 ). Showing its polybasic properties, $\mathbf{3 4}$ reacts with pyrrole to generate two deprotonated pyrrole molecules within $\left[\left\{\left(\mathrm{Ph}_{2} \mathrm{Si}\left(\mathrm{NAr}^{*}\right)\left(\mathrm{NHAr}^{*}\right)\right) \mathrm{Mg}\left(\mathrm{NC}_{4} \mathrm{H}_{4}\right)_{2}(\mathrm{THF}) \mathrm{Na}(\mathrm{THF})_{2}\right\}\right]$ which has a contacted ion pair structure, while 2,6-diisopropylaniline mainly gave another SSIP product $\left[\left\{\mathrm{Na}(\mathrm{THF})_{6}\right\}^{+}\left\{\left(\mathrm{Ph}_{2} \mathrm{Si}\left(\mathrm{NAr}^{*}\right)_{2}\right) \mathrm{Mg}\left(\mathrm{NHAr}{ }^{*}\right)(\mathrm{THF})\right\}^{-}\right](37)$ in a mixture of products. The pyrrole reaction is noteworthy since both the butyl group and one amido arm of the silyl(bisamido) ligands carry out the twofold deprotonation. ${ }^{66}$

Scheme 30. Representative reactions of the sodium [silyl(bisamido)]-alkylmagnesiate complex $\left[\left\{\mathrm{Na}(\mathrm{THF})_{6}\right\}^{+}\left\{\left(\mathrm{Ph}_{2} \mathrm{Si}\left(\mathrm{NAr}^{*}\right)_{2}\right) \mathrm{Mg}(\mathrm{Bu})(\mathrm{THF})\right\}^{-}\right], 34$.
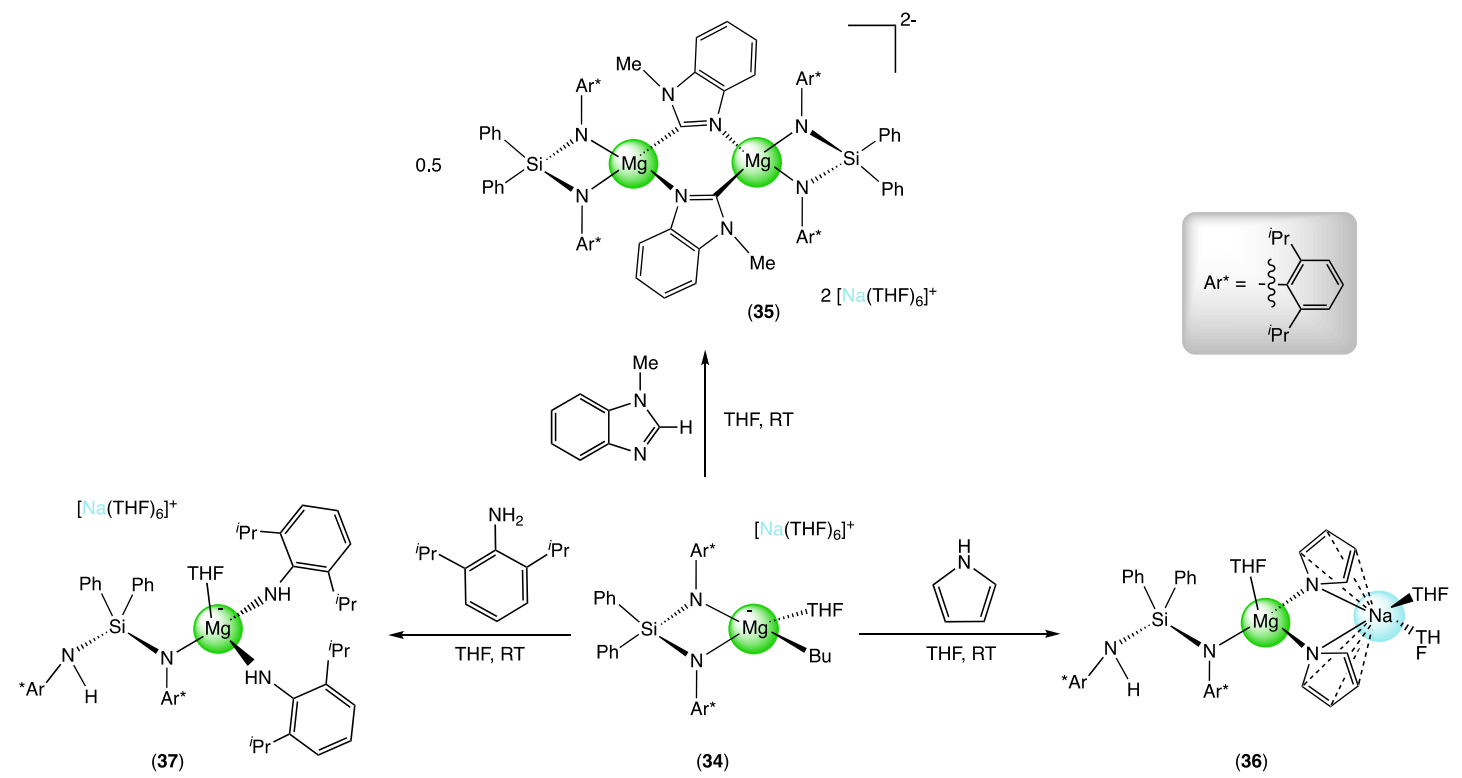
The sodium butylmagnesiate $\mathbf{3 4}$ can also function as a SET (single electron transfer) reagent. Its reaction with quinoxaline $(Q x)$, produces the blue crystalline complex $\left[\left\{\mathrm{Na}(\mathrm{THF})_{6}\right\}^{+}\right]_{2} \quad\left[\left\{\mathrm{Ph}_{2} \mathrm{Si}\left(\mathrm{NAr}^{*}\right)_{2}\right\}_{2} \mathrm{Mg}_{2}(\mathrm{Qx})_{2}\right]^{2-}$ 38, whose charge-separated structure shows two quinoxalyl radical anions Qx• stabilized within a dimeric magnesiate framework (Scheme 31).

Scheme 31. Top: Molecular structure of the $\left[\left\{\mathrm{Ph}_{2} \mathrm{Si}\left(\mathrm{NAr}^{*}\right)_{2}\right\}_{2} \mathrm{Mg}_{2}(\mathrm{Qx})_{2}\right]^{2-}$ anionic moiety of 38. Bottom: Reactions of $\left[\left\{\mathrm{Na}(\mathrm{THF})_{6}\right\}^{+}\left\{\left(\mathrm{Ph}_{2} \mathrm{Si}\left(\mathrm{NAr}{ }^{*}\right)_{2}\right) \mathrm{Mg}(\mathrm{Bu})(\mathrm{THF})\right\}^{-}\right] 34$ with quinoxaline $(\mathrm{Qx})$ and $\mathrm{TMP}(\mathrm{H})$ to give $\mathbf{3 8}$ and 39 respectively. 


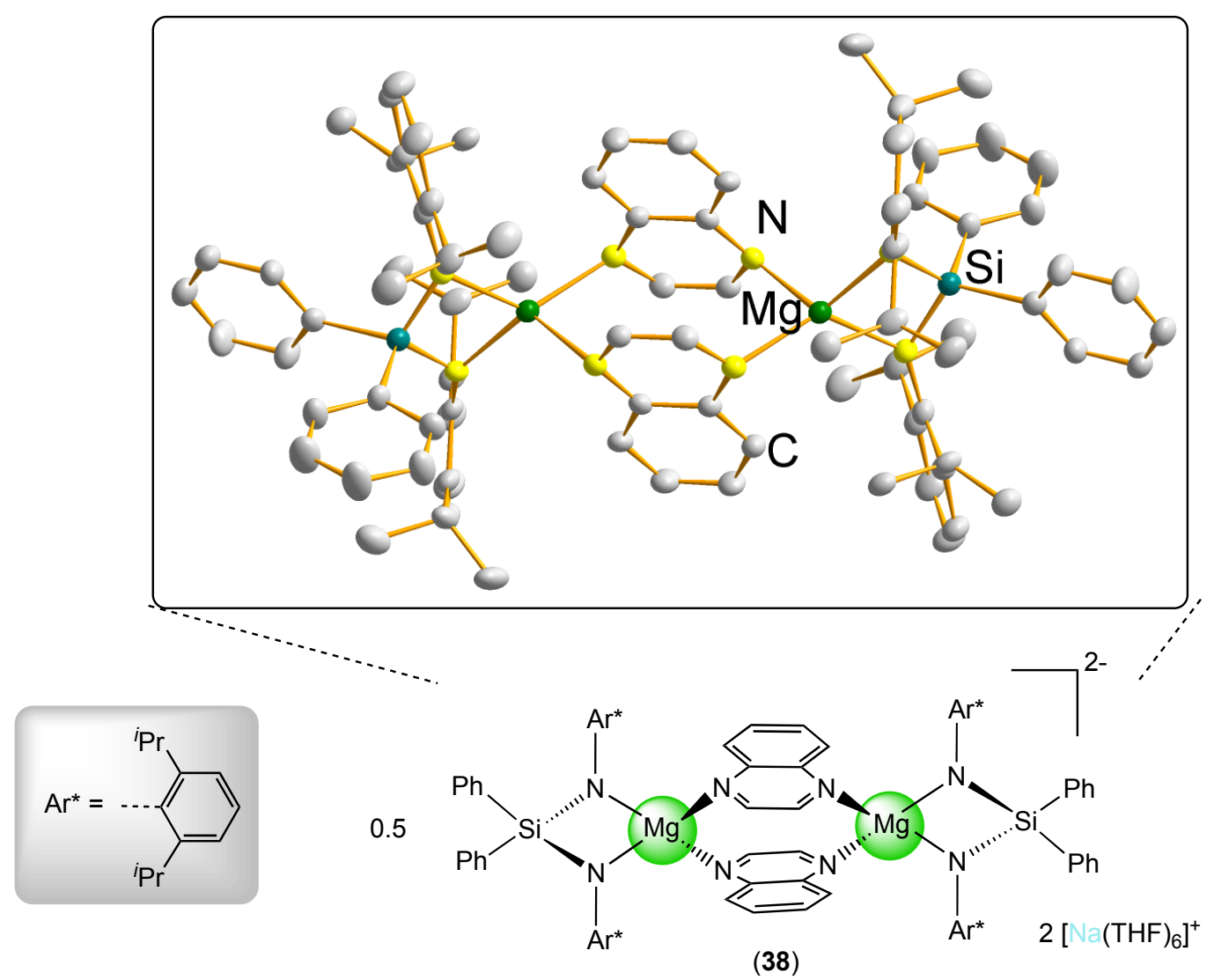<smiles>Cc1nc2ccccc2nc1CC(=O)I</smiles>

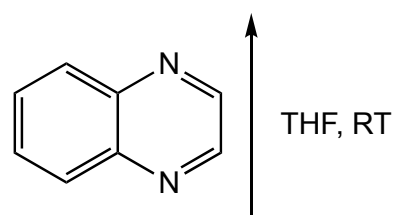

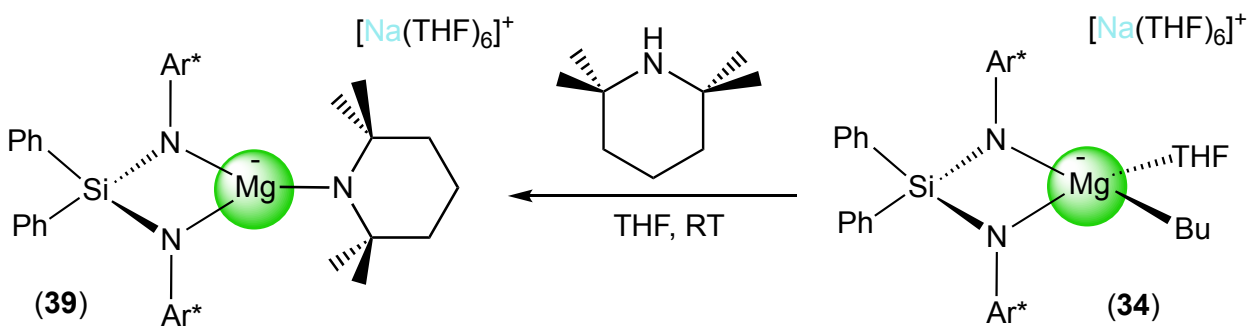

The radical character of the anionic quinaxolyl ligand was confirmed by EPR spectroscopy in THF solution. The analogous amide complex $\left[\mathrm{Na}(\mathrm{THF})_{6}\right]^{+}$ $\left[\left\{\mathrm{Ph}_{2} \mathrm{Si}\left(\mathrm{NAr}^{*}\right)_{2}\right\} \mathrm{Mg}(\mathrm{TMP})\right]^{-}(39)$, also produced 38 when mixed with quinoxaline. The important role of the $\left\{\mathrm{Ph}_{2} \mathrm{Si}\left(\mathrm{NAr}^{*}\right)_{2}\right\}^{2-}$ steric stabilizer in radical formation was highlighted in the corresponding reaction with the aforementioned sodium all-alkyl 
magnesiate $\mathrm{NaMg}\left(\mathrm{CH}_{2} \mathrm{SiMe}_{3}\right)_{3} 29$ that led instead to the chemoselective $\mathrm{C} 2$ alkylation of this heterocycle $(\mathbf{4 0}$, Scheme 32$) .{ }^{67}$

Scheme 32. Chemoselective C2 alkylation of quinoxaline.

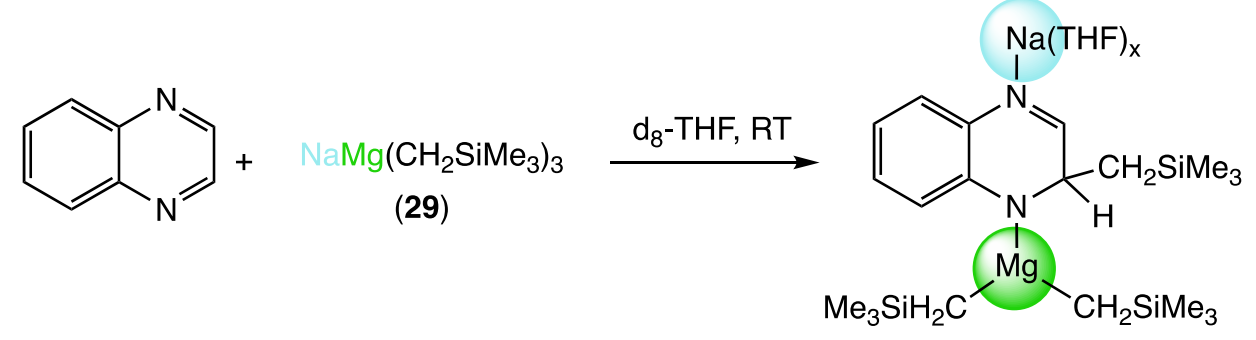

(40)

Another heteroleptic sodium magnesiate base [(TMEDA) $\left.\mathrm{Na}(\mathrm{TMP}){ }_{2} \mathrm{Mg}\left(\mathrm{CH}_{2} \mathrm{SiMe}_{3}\right)\right](41)$ has been used to effect AMMMg of three $\mathrm{N}$ alkylated indoles at room temperature. The alkyl substituent plays an important role in the rate of reaction since when it is iso-propyl the reaction needs 16 hours for completion to give the monoindol-2-yl complex [(TMEDA) Na(TMP)( $\alpha$ $\left.\left.\mathrm{C}_{11} \mathrm{H}_{12} \mathrm{~N}\right) \mathrm{Mg}(\mathrm{TMP})\right]$ (42) with deprotonation at the $\mathrm{C} 2$ position. With smaller Me and Et substituents, magnesiation is rapid (completed in 15 minutes) and the reduction in steric factors enables a more atom efficient fourfold deprotonation process affording the disodium tetraindol-2-yl magnesiates $\left[\mathrm{Na}(\mathrm{TMEDA})_{2} \mathrm{Mg}\left(\alpha-\mathrm{C}_{9} \mathrm{H}_{8} \mathrm{~N}\right)_{4}\right]$ and $\left[\mathrm{Na}(\mathrm{TMEDA})_{2} \mathrm{Mg}\left(\alpha-\mathrm{C}_{10} \mathrm{H}_{11} \mathrm{~N}\right)_{4}\right]$, which have classical Weiss motifs (43 and $\mathbf{4 4}$, Scheme 33). Since the 1:1 stoichiometry of these last two reactions was not correct for the tetraindol-2-ylmagnesiates formed, the reactions were repeated using the stoichiometrically correct 2:1:4 mixture of $\mathrm{NaTMP}, \mathrm{Mg}\left(\mathrm{CH}_{2} \mathrm{SiMe}_{3}\right)_{2}$ and indole leading to $81 \%$ and $70 \%$ yields of $\mathbf{4 3}$ and $\mathbf{4 4}$ respectively. In situ iodine quenches and Pd- 
catalysed cross-coupling reactions with iodobenzene of these three indol-2-yl magnesiates were successful with yields spanning the range $66-82 \% .{ }^{68}$

Scheme 33. Contrasting alkali-metal-mediated reactions of [(TMEDA) $\left.\mathrm{Na}(\mathrm{TMP})_{2} \mathrm{Mg}\left(\mathrm{CH}_{2} \mathrm{SiMe}_{3}\right)\right](41)$ with $\mathrm{N}$-alkylated indoles and a stochiometriccorrect variant reaction.

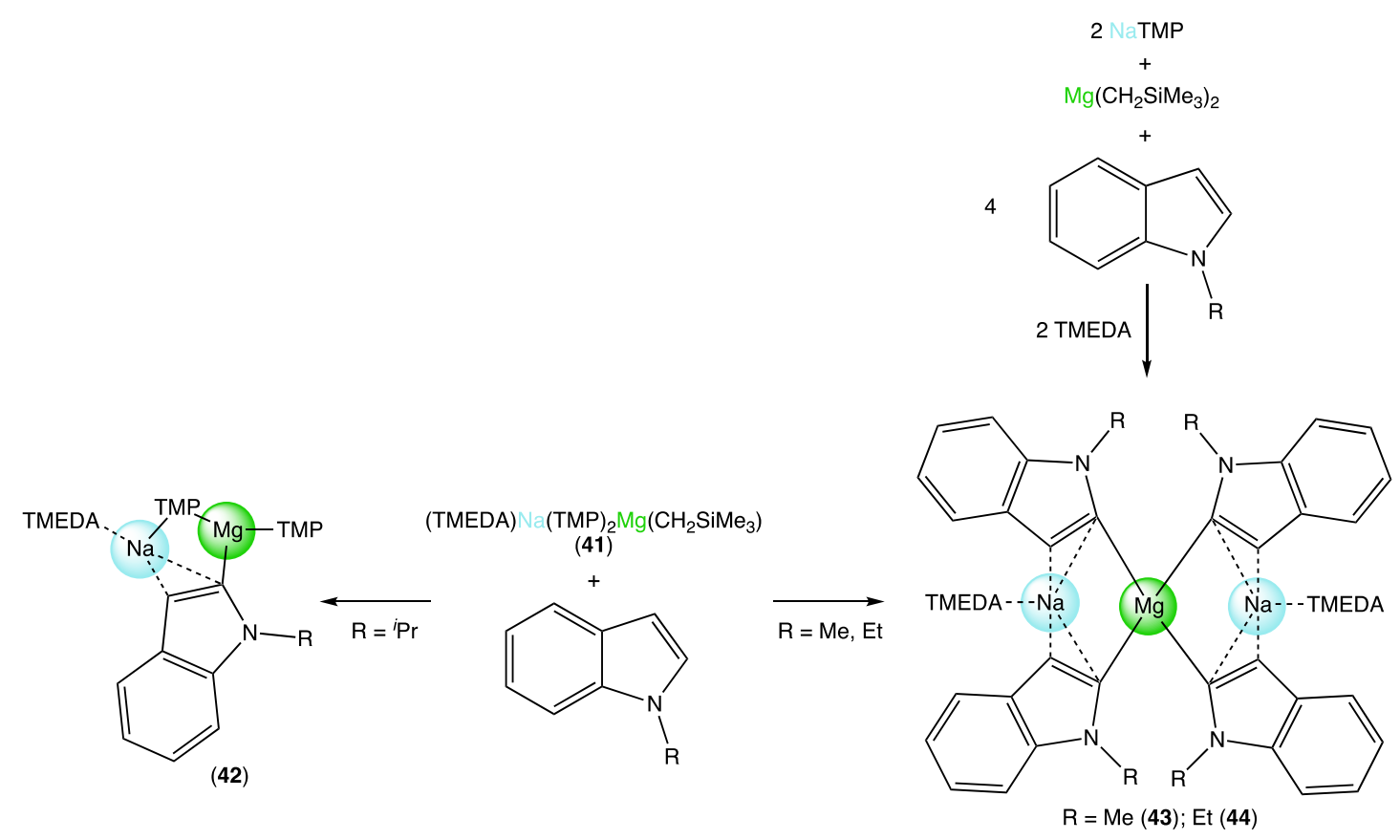

\subsubsection{Magnesium and Calcium Chemistry Involving Dianionic Butadienyl Ligands}

Whereas the pre-inverse-crown reagents generally involve two distinct metals (one an alkali metal) combined with a set of monoanionic ligands, other mixed-metal complexes that exhibit synergistic effects are known where the two metals are part of a system containing a dianionic ligand. Homometallic 1,4-dilithio 1,3-butadienes come into this category. These have provided access to distinct types of unusual compounds including magnesiacyclopentadienes, spirodilithio magnesiacyclopentadienes, and dimagnesiabutadiene. Magnesiacyclopentadienes (e.g. 45) are made by a metathesis reaction from a 1:1 mixture of 1,4-dilithio 1,3-butadienes and $\mathrm{MgCl}_{2}$ in ether solution; 
while altering the stoichiometry to 2:1 generates novel spiro-dilithio magnesiacyclopentadiene (46, Scheme 34$)$. The inset shows the molecular structure of a spiro-dilithio magnesiacyclopentadiene-TMEDA complex. Changing from the halide salt $\mathrm{MgCl}_{2}$ to the Grignard reagent ${ }^{i} \mathrm{PrMgCl}$ produces a dimagnesiabutadiene in THF solution (47, Scheme 34$)$.

Scheme 34. Representative reactions of 1,4-dilithio 1,3-butadienes, with the molecular structure of a spiro-dilithio magnesiacyclopentadiene-TMEDA complex (46) shown in the inset.
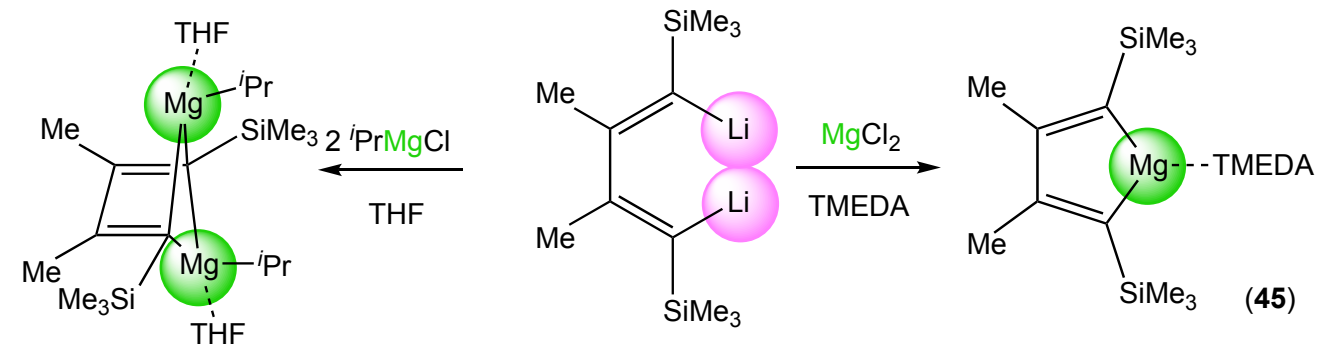

(47)

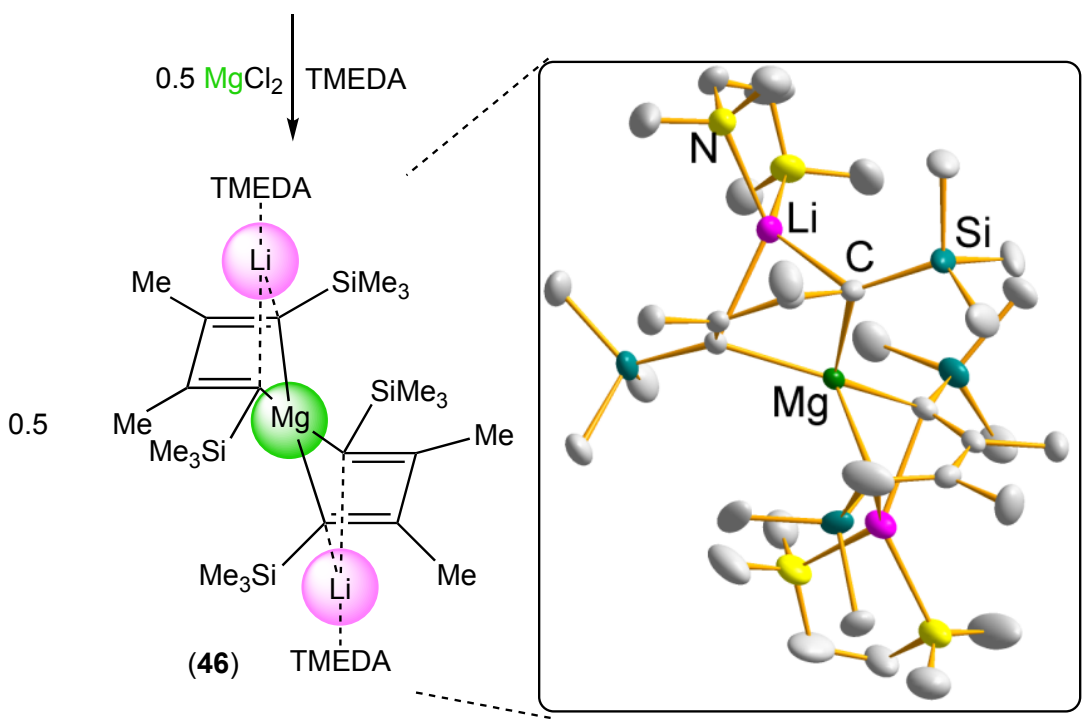

Access to aminocyclopentadienes has proved possible starting from diiodo butadienes and reacting the metallo intermediates with thioformamides (Scheme 35). ${ }^{69}$

Scheme 35. Preparation of aminocyclopentadienes from diiodo butadienes. 
<smiles>[R]C(=C(C)C(C)(C)C)/C([R])=C(\[R])I</smiles><smiles>[R]C1=C([R])C([Y17])C([R])=C1[R]</smiles><smiles>[R]C(I)=C([R])c1ccccc1I</smiles><smiles>[R]C1=C([R])C([NH2+])c2ccccc21</smiles>

Several open butadienyl organomagnesium complexes have also been accessed from 1,4-dilithio butadiene precursors. Partial transmetalation leads to 1-Li-4-(MgR)butadienes (48), while full transmetalation gives 1,4-bis(MgR)-butadienes (e.g. 49) or in the presence of magnesium chloride, $1-\mathrm{Mg}^{*}-4-(\mathrm{MgR})$-butadiene, where $\mathrm{Mg}^{*}$ represents a $\mathrm{Mg}_{2} \mathrm{Cl}_{3}$ unit (50, Scheme 36). ${ }^{70}$

Scheme 36. Preparation of open butadienyl organomagnesium complexes $\mathbf{4 8 - 5 0}$ from 1,4-dilithio butadiene precursors. 

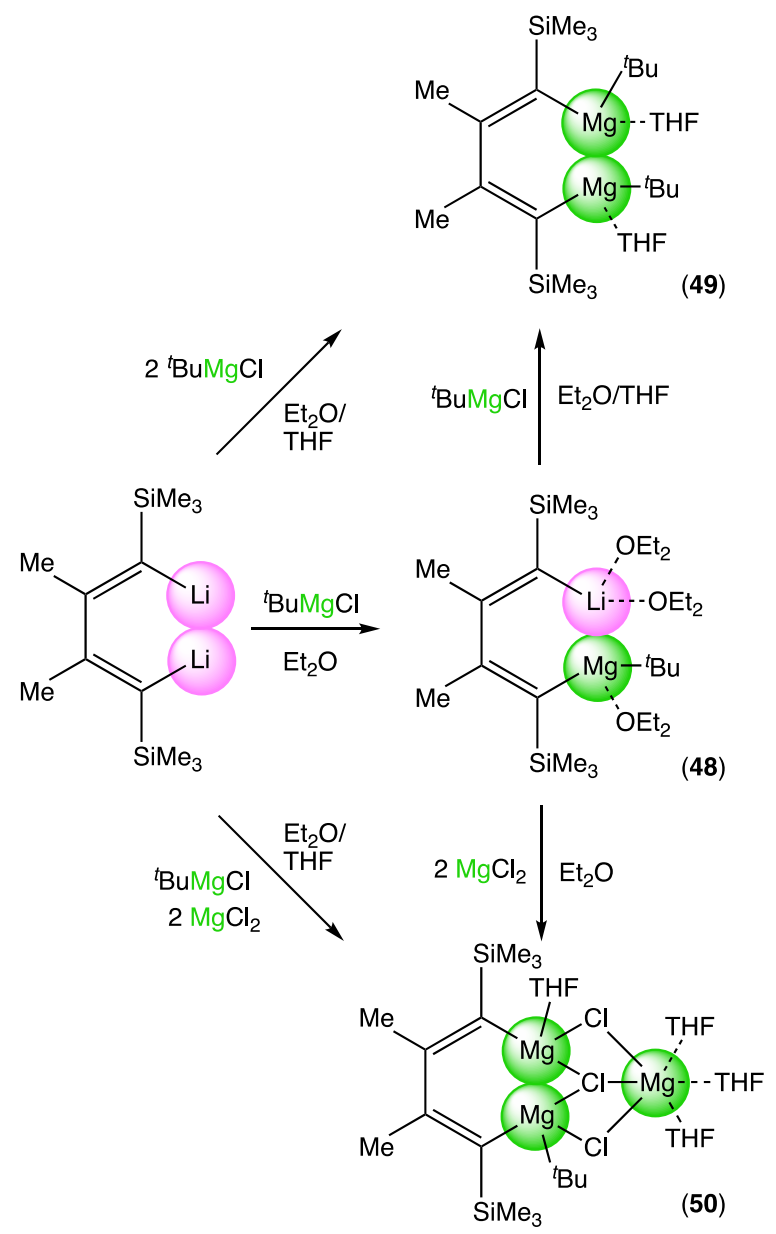

A 2,3-phenyl substituted 1,4-dilithio 1,3-butadiene has also been used as a precursor to organocalcium species, though significantly not calcium-based cyclopentadienes. ${ }^{71}$ 1-Calcio-4-lithio-1,3-butadiene (51) was formed by a 1:1 metathesis reaction with calcium iodide in THF solution, which upon standing in THF for a day produced an indenyllithium product (52). Increasing the stoichiometry of the salt to two equivalents with a 2,3-dimethyl substituted dilithio species put two calcium centers on the open butadienyl framework in a mixture of two distinct products (Scheme 37), namely a homometallic dicalcio diiodide derivative (53) and a heterometallic dimeric dicalcio-monolithio triiodide derivative (54). 
Scheme 37. Reactions and structures in 1,4-dimetallo 1,3-butadiene chemistry involving calcium.

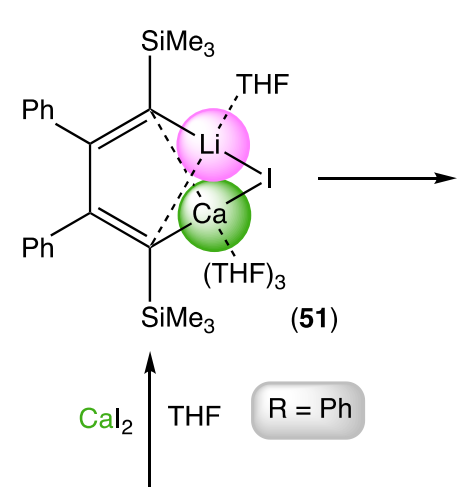<smiles>[2H]c1c(SC)ccc(C(C)(C)C)c1[2H]</smiles>

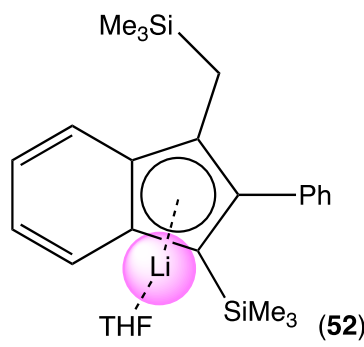

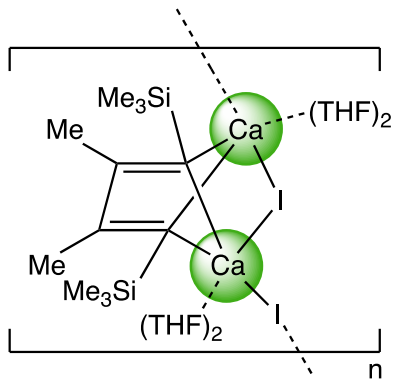

(53)

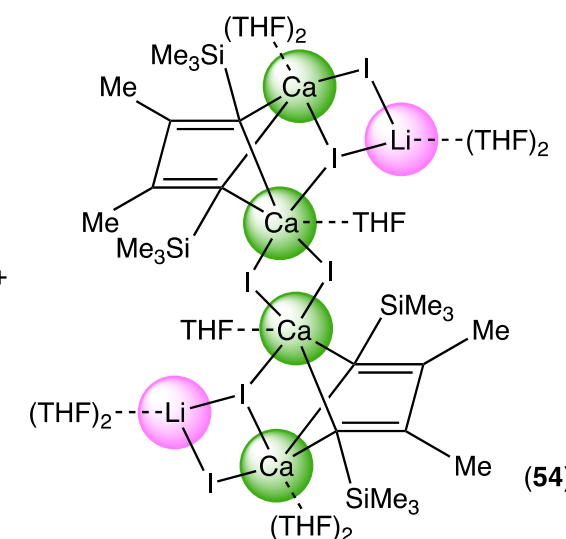<smiles>[Y]C(C)C1CCCO1</smiles>

$(\mathrm{THF})_{2}$ (54)

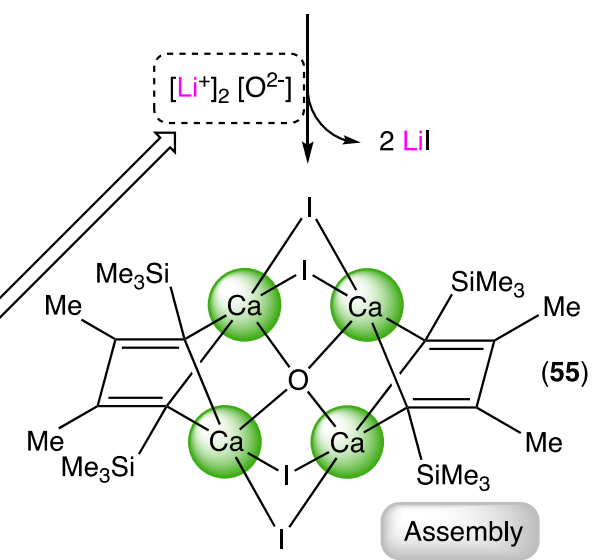

The former can be considered a heavy Grignard reagent. The latter can be regarded as a 2:1 cocomplex between RCal and the other product of the metathesis Lil and so bears some connection to the synthetically prolific turbo-Grignard reagents $\mathrm{RMgCl} \cdot \mathrm{LiCl}(\mathrm{R}=$ alkyl or amido) described in Section 2.3 of this review. Interestingly, this product mixture decomposes THF to generate $\mathrm{a} \mathrm{Ca}_{4}$ inverse crown ether complex (55), a mechanism for which has been postulated (Scheme 37), built on earlier work 
that documented the cleavage of THF by bimetallic sodium-magnesium and related bimetallic systems. ${ }^{72}$

\subsubsection{Heterobimetallic Hydrides}

Recently molecular forms of s-block hydrides have attracted increasing interest due to their involvement as possible intermediates in stoichiometric and catalytic bondforming reactions, while lighter congeners are sometimes cited as relevant to hydrogen-storage technology as a result of their relatively high hydrogen weight percentages. Heterobimetallic examples are a focal point of this activity. A study of reactions between the potassium bisamido-alkylmagnesiate [ $\left.\mathrm{K}\left\{\mathrm{N}\left(\mathrm{SiMe}_{3}\right)_{2}\right\}_{2} \mathrm{Mg}^{n} \mathrm{Bu}\right]$ (56) and phenylsilane $\left(\mathrm{PhSiH}_{3}\right)$ uncovered selective alkyl metathesis producing the silane $\mathrm{PhSi}(\mathrm{H})_{2}{ }^{n} \mathrm{Bu}$ and the hydrido inverse crown $\left[\mathrm{K}_{2}\left\{\mathrm{~N}\left(\mathrm{SiMe}_{3}\right)_{2}\right\}_{4} \mathrm{Mg}_{2}(\mathrm{H})_{2}\right]$ comprising an 8-membered ( $\mathrm{KNMgN})_{2}$ host ring with two bridging hydrides in the core (Scheme 38). ${ }^{73}$

Scheme 38. Formation of a hydrido inverse crown (57) via a silane-induced alkyl metathesis.

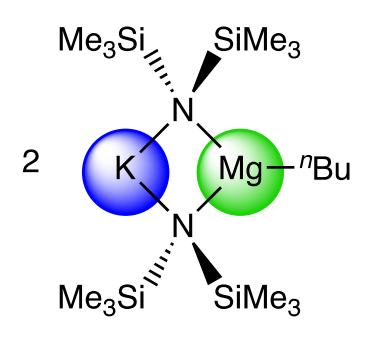

(56)

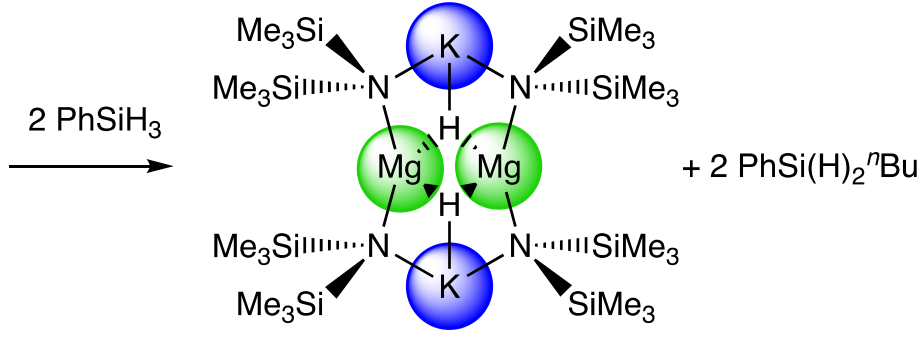

(57)

Hydrido inverse crowns were first synthesized indirectly via $\beta$-hydride elimination from metal diisopropylamide species ${ }^{74}$ so this silane approach represented a new 
direct preparative method. Reaction of phenylsilane with the sodium congener $\left[\mathrm{Na}\left\{\mathrm{N}\left(\mathrm{SiMe}_{3}\right)_{2}\right\}_{2} \mathrm{Mg}{ }^{n} \mathrm{Bu}\right]$ (58) produced the remarkable heterododecametallic polyhydride $\quad\left[\mathrm{Mg}_{6} \mathrm{Na}_{6}\left\{\mathrm{~N}\left(\mathrm{SiMe}_{3}\right)_{2}\right\}_{8} \mathrm{H}_{10}\right] \quad$ (59), comprising eight 1,1,1,3,3,3hexamethyldisilazide (HMDS) and ten hydride ligands (Figure 17). This represented a new approach to higher metal hydride clusters and established that $\mathrm{M}-\mathrm{N} / \mathrm{Si}-\mathrm{H} \sigma-b o n d$ metathesis as well as $\mathrm{Mg}-\mathrm{C} / \mathrm{Si}-\mathrm{H} \sigma$-bond metathesis was taking place.

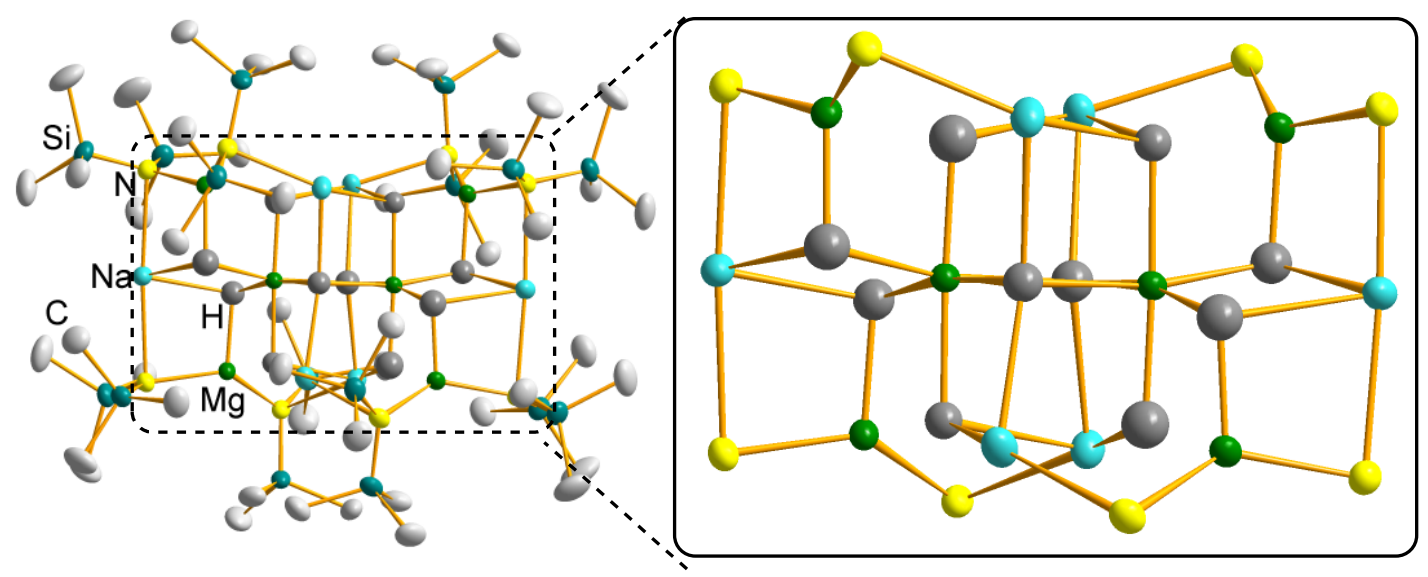

Figure 17. Molecular structure of the heterododecametallic polyhydride $\left[\mathrm{Mg}_{6} \mathrm{Na}_{6}\left\{\mathrm{~N}\left(\mathrm{SiMe}_{3}\right)_{2}\right\}_{8} \mathrm{H}_{10}\right], 59$.

A novel bimetallic trinuclear compound the salient feature of which is a bulky ambidentate Schiff-base ligand in an anionic unit containing both magnesium and sodium (62) has been established as an efficient catalyst for hydrosilylation of a selection of ketones with the activated silane $(\mathrm{EtO})_{3} \mathrm{SiH}$. Made by reacting elemental sodium with the Grignard-type reagent analog $\mathbf{6 1}$, this bimetallic derivative in a 10 mol\% loading hydrosilylates acetophenone in $93 \%$ yield after 2 hours at $25{ }^{\circ} \mathrm{C}$ giving a mixture of $\mathrm{Si}(\mathrm{OCHMePh})_{x}(\mathrm{OEt})_{4-x}(x=1-4)$ products. Note no mechanistic details were uncovered in the study. Demonstrating a strong synergistic effect, neither the monometallic homoleptic magnesium complex 60 nor its Grignard reagent analog (61) 
come close to this bimetallic efficiency in both reaching $90 \%$ yield but only after 22 hours at $70{ }^{\circ} \mathrm{C}$ (scheme 39). ${ }^{75}$

Scheme 39. Catalytic hydrosilylation of acetophenone using a sodium magnesiate catalyst (62) that outperforms its homometallic neutral magnesium and Grignard reagent analogs 60 and 61 .

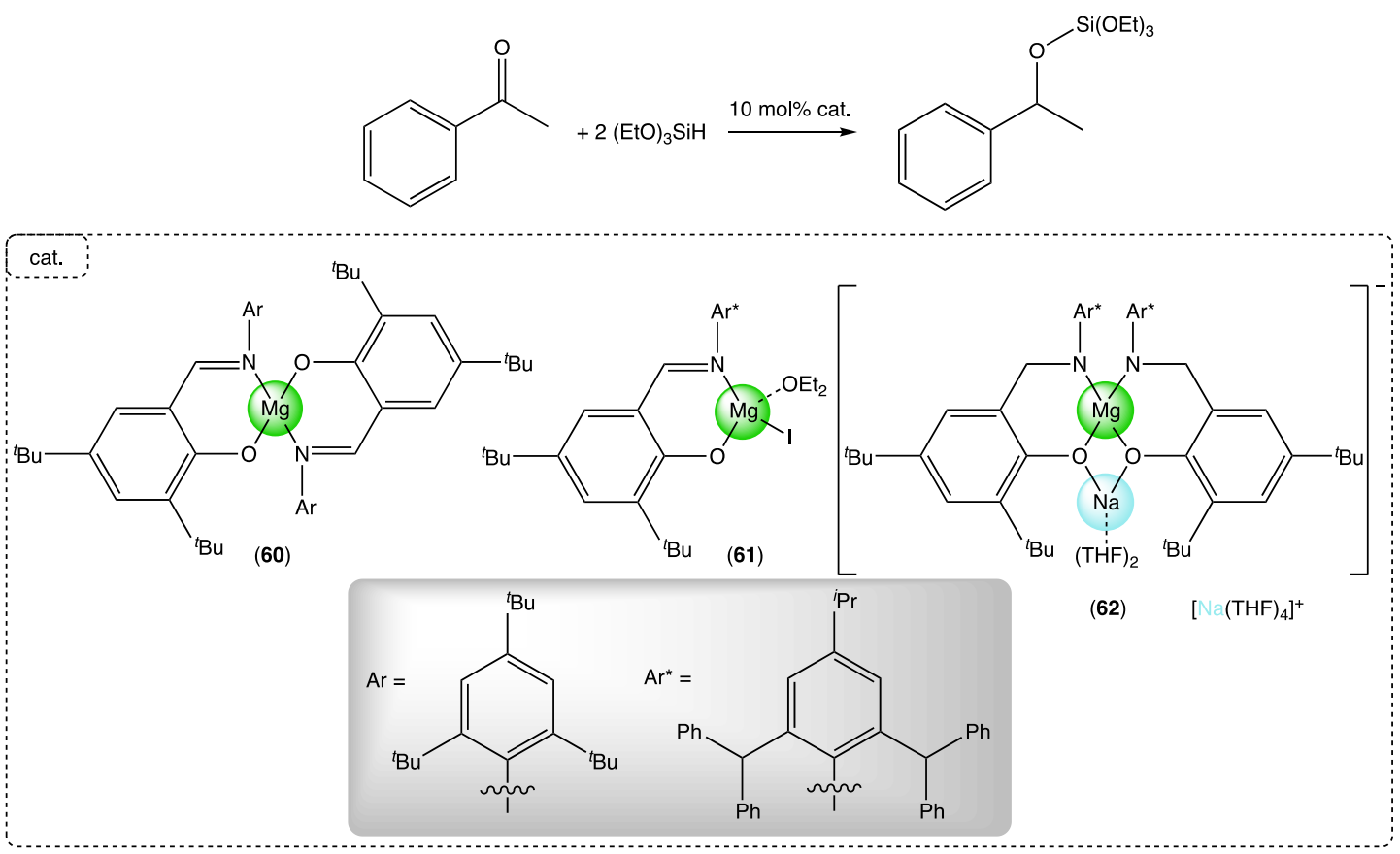

\subsubsection{Magnesiate and Related Complexes Synthesized via Di- $n$-butylmagnesium}

Di- $n$-butylmagnesium has been utilized in the preparation of donor solvates of lithium $\operatorname{tris}(n$-butyl)magnesiates by a simple cocomplexation approach. In general, these reactions produce predictable structures with Weiss motifs as observed in the (anion) homoleptic $\left[\{(R, R)-\mathrm{TMCDA}\} \mathrm{Li}\left({ }^{n} \mathrm{Bu}\right)_{2} \mathrm{Mg}\left({ }^{n} \mathrm{Bu}\right)\right]_{2}(63)$, but in the presence of an alcohol a heteroleptic variant with an alternative ladder motif can be accessed in $\left[(T M E D A) L i\left({ }^{n} \mathrm{Bu}\right)\left(\mathrm{O}^{n} \mathrm{Bu}\right) \mathrm{Mg}\left({ }^{n} \mathrm{Bu}\right)\right]_{2},(64$, Scheme 40$) \cdot{ }^{76}$ 
Scheme 40. Preparation and structure of a lithium tris(n-butyl)magnesiate (63, RHS) and a lithium bis(n-butyl)(n-butoxy)magnesiate (64, LHS).

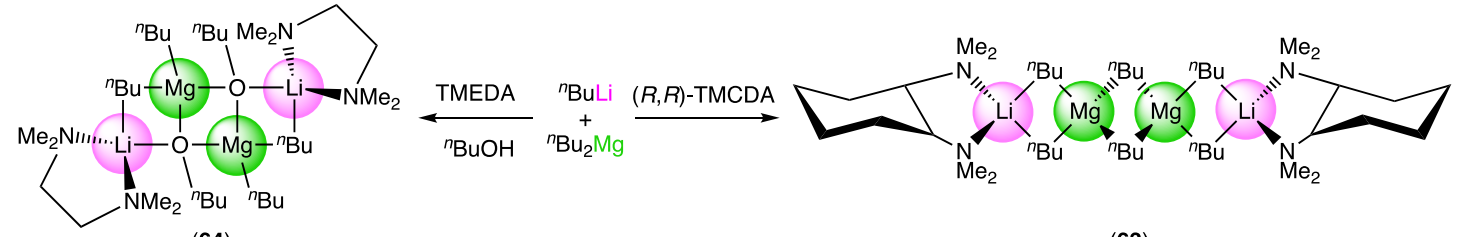

(64)

(63)

When mixed together in hexane, $n$-butyllithium and di-n-butylmagnesium react with the aminopyridine 2-Me $\mathrm{MiNH}_{3}-6-\mathrm{MeC}_{5} \mathrm{H}_{3} \mathrm{~N}\left(\mathrm{~L}^{1} \mathrm{H}\right)$ to produce the mixed lithiummagnesium polymer $\left[\left(\mathrm{L}^{1}\right) \mathrm{Li}\left(\mu^{2}{ }^{n} \mathrm{Bu}\right) \mathrm{Mg}\left(\mathrm{L}^{1}\right)\right]_{\infty}(65$, scheme 41$)$. The polymer propagates through an unusual $\mathrm{Mg}-{ }^{n} \mathrm{Bu}-\mathrm{Li}$ bridge. Introducing the donor solvent THF forms a more predictable magnesiate complex in $\left(\mathrm{L}^{1}\right)_{3} \mathrm{MgLi}(\mathrm{THF}), 66 .{ }^{77}$

Scheme 41. Solvent-dependent preparations and structures of lithium amidomagnesiates 65 and 66 .

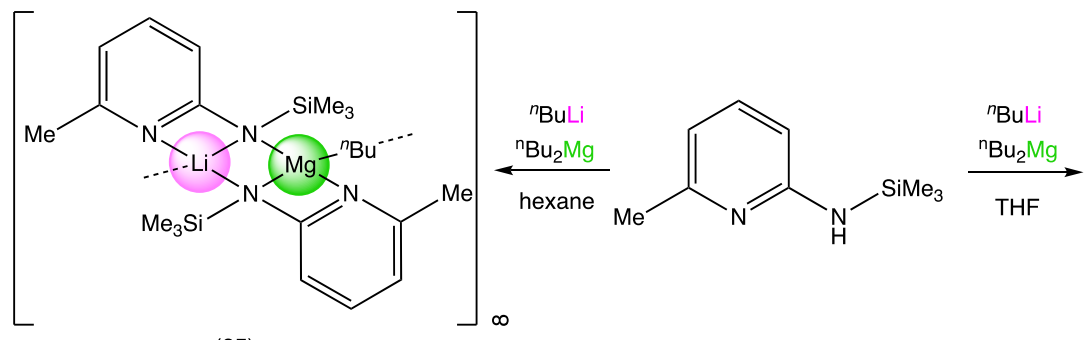

(65)

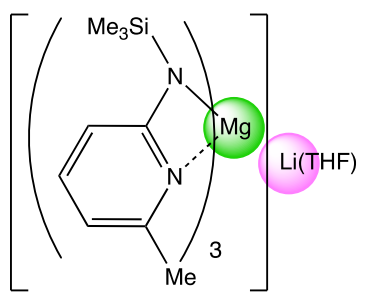

(66)

Reaction of a 1:1:3 stoichiometric mixture of benzylpotassium, di- $n$-butylmagnesium and diphenylamine in a solvent mixture composed of hexane, THF and PMDETA, has produced a rare example of a potassium tris(amido)magnesiate in (PMDETA)K( $\mu$ $\left.\mathrm{NPh}_{2}\right) \mathrm{Mg}(\mathrm{THF})\left(\mathrm{NPh}_{2}\right)_{2}(67)$. In the crystal, the $\sigma-\pi$ bonding distinction commonly found in bimetallic alkali metal magnesiates is clearly present with "hard" Mg $\sigma$-bonded to three $\mathrm{N}$ and $\mathrm{O}$ atoms; while the "soft" $\mathrm{K}$ center binds to $4 \mathrm{~N}$ atoms but engages with two aryl rings in $\eta^{6}$ and $\eta^{2}$ aryl- $\pi$ interactions (Figure 18). Looking for possible 
synergistic effects, this paper reports that the prevalence of such $\mathrm{K}-\mathrm{C}$ aryl- $\pi$ interactions appears not to be as significant in structurally characterized neutral potassium diphenylamide solvates. ${ }^{78}$

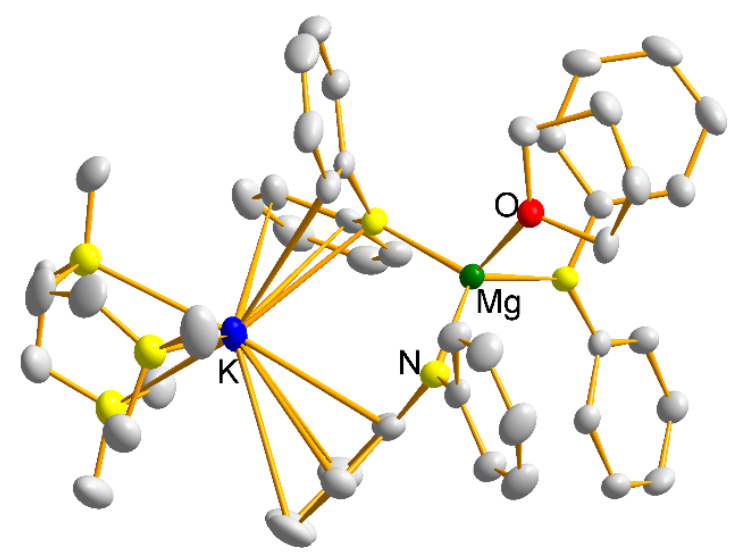

Figure 18. Molecular structure of $(\mathrm{PMDETA}) \mathrm{K}\left(\mu-\mathrm{NPh} \mathrm{h}_{2}\right) \mathrm{Mg}(\mathrm{THF})\left(\mathrm{NPh}_{2}\right)_{2}(67)$.

Hexamethyldisilazide features in the unsolvated polymeric sodium alkylmagnesiate $\left[\mathrm{Na}(\mathrm{HMDS})_{2} \mathrm{Mg}\left({ }^{n} \mathrm{Bu}\right)\right]_{\infty}(68) \cdot{ }^{79}$ Its acceptor reactions with a variety of donors have been studied. Most interesting result is the synthesis and characterization of novel "inverse magnesiates" $\left[\{\mathrm{Na}(\mathrm{HMDS})\}_{2} \mathrm{Mg}\left({ }^{n} \mathrm{Bu}\right)_{2} \text { (donor) }\right]_{\infty}[$ where donor $=\mathrm{TMEDA}, 69 ;$ or $(R, R)-$ TMCDA, 70] (scheme 42).

Scheme 42. Preparation and structure of the inverse magnesiate $\left.\left[\{\mathrm{Na}(\mathrm{HMDS})\}_{2} \mathrm{Mg}\left({ }^{n} \mathrm{Bu}\right)_{2}(R, R)-\mathrm{TMCDA}\right)\right]_{\infty}(70)$.

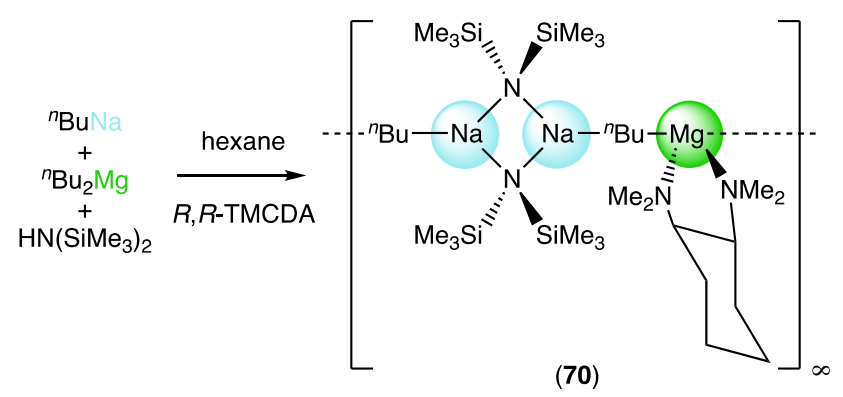


This inverse categorization acknowledges a reversal of the common Lewis acidic, Lewis basic distributions in magnesiates. Normally the stronger Lewis acidic metal (Mg) attracts the more Lewis basic ligands (that is, anions), but here the weaker Lewis acidic $\mathrm{Na}$ coordinates to four anions, whereas $\mathrm{Mg}$ coordinates to two anions and two $\mathrm{N}$ donor atoms. Thus, the di- $n$-butylmagnesium unit is functioning as a Lewis base to solvate the NaHMDS dimer (and concomitantly the NaHMDS dimer operates as a Lewis acid). Dimers of NaHMDS with more conventional donors such as THF ${ }^{80}$ are well known. This inverse magnesiation can be attributed to the stability of the fourmembered $(\mathrm{NaN})_{2}$ ring. The zincate $\left[\{\mathrm{Li}(\mathrm{HMDS})\}_{2} \mathrm{Zn}(\mathrm{Me})_{2}(\mathrm{TMEDA})\right]_{\infty}(\mathbf{7 1})$ has also been interpreted in this inverse category. ${ }^{81}$ Demonstrating further the stability imparted on ate complexes by the HMDS ligand, the heteroleptic potassium calcium ate complex $\left[\left\{\mathrm{K}^{2}-\left(\mathrm{Im}^{t \mathrm{Bu}} \mathrm{NCONPh}\right) \mathrm{Ca}\left\{\mathrm{N}\left(\mathrm{SiMe}_{3}\right)_{2}\right\}\left\{\mathrm{KN}\left(\mathrm{SiMe}_{3}\right)_{2}\right\}\right]_{\infty}\right.$ (where $\mathrm{Im}^{t \mathrm{Bu}} \mathrm{NCONPh}$ is imidazol-2ylidene- $N$ '-phenylureate, 72 ) has been made by a metathesis approach (Scheme 43). This polymeric ate can be regarded as a cocomplex between KHMDS and $\left[\left(\mathrm{Im}^{\mathrm{tBu}} \mathrm{NCONPh}\right) \mathrm{Ca}\left\{\mathrm{N}\left(\mathrm{SiMe}_{3}\right)_{2}\right\}\right] .82$

Scheme 43. Preparation and structure of the polymeric potassium calciate complex of $\left[\left\{\mathrm{K}^{2}-\left(\mathrm{Im}{ }^{t \mathrm{Bu}} \mathrm{NCONPh}\right) \mathrm{Ca}\left\{\mathrm{N}\left(\mathrm{SiMe}_{3}\right)_{2}\right\}\left\{\mathrm{KN}\left(\mathrm{SiMe}_{3}\right)_{2}\right\}\right]_{\infty}(\mathbf{7 2})\right.$.

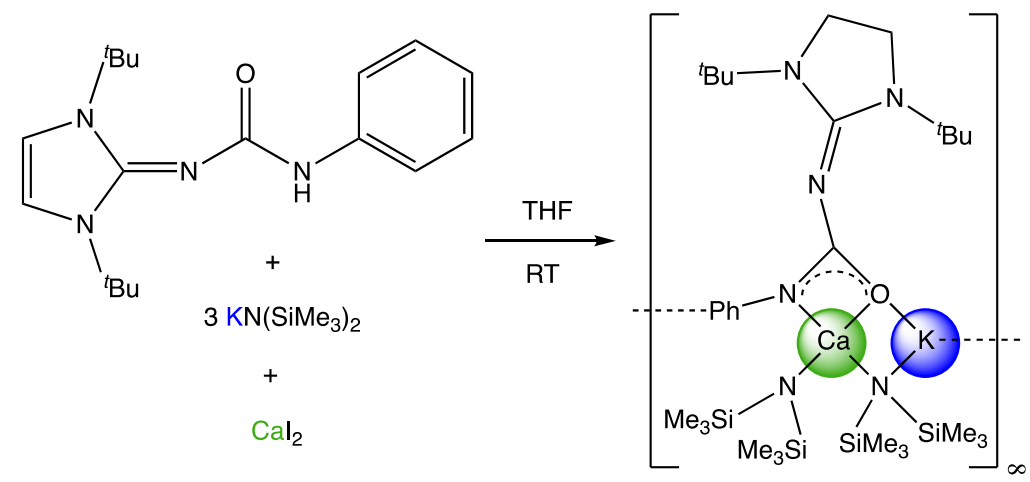

(72) 


\subsubsection{Applications in Homogeneous Catalysis and Polymerization Chemistry}

Reaction of four molar equivalents of potassium 2,6-diisopropylphenylamide with calcium iodide in THF has produced the higher order calciate complex $\left[\mathrm{K}_{2} \mathrm{Ca}\{\mathrm{N}(\mathrm{H}) \mathrm{Dipp}\}_{4}\right]_{\infty}(\mathbf{7 3}$, Scheme 44$) .{ }^{83}$ This ate crystallized from a THF/toluene mixture as a donor solvent free coordination polymer. Propagation of the structure is through Ca-N and $\mathrm{K}-\pi$-aryl interactions with the calcium center occupying a low fourcoordinate distorted Nx4 tetrahedral arrangement. This calciate was found to catalyse hydroamination of diphenylbutadiyne with 2,6-diisopropylaniline but a cascade process occurs subsequently leading to formation of a tetracyclic imine (Scheme 44).

Scheme 44. Catalytic hydroamination of diphenylbutadiyne with 2,6diisopropylaniline ultimately producing a tetracyclic imine. Structure of catalyst $\mathbf{7 3}$ shown in inset.
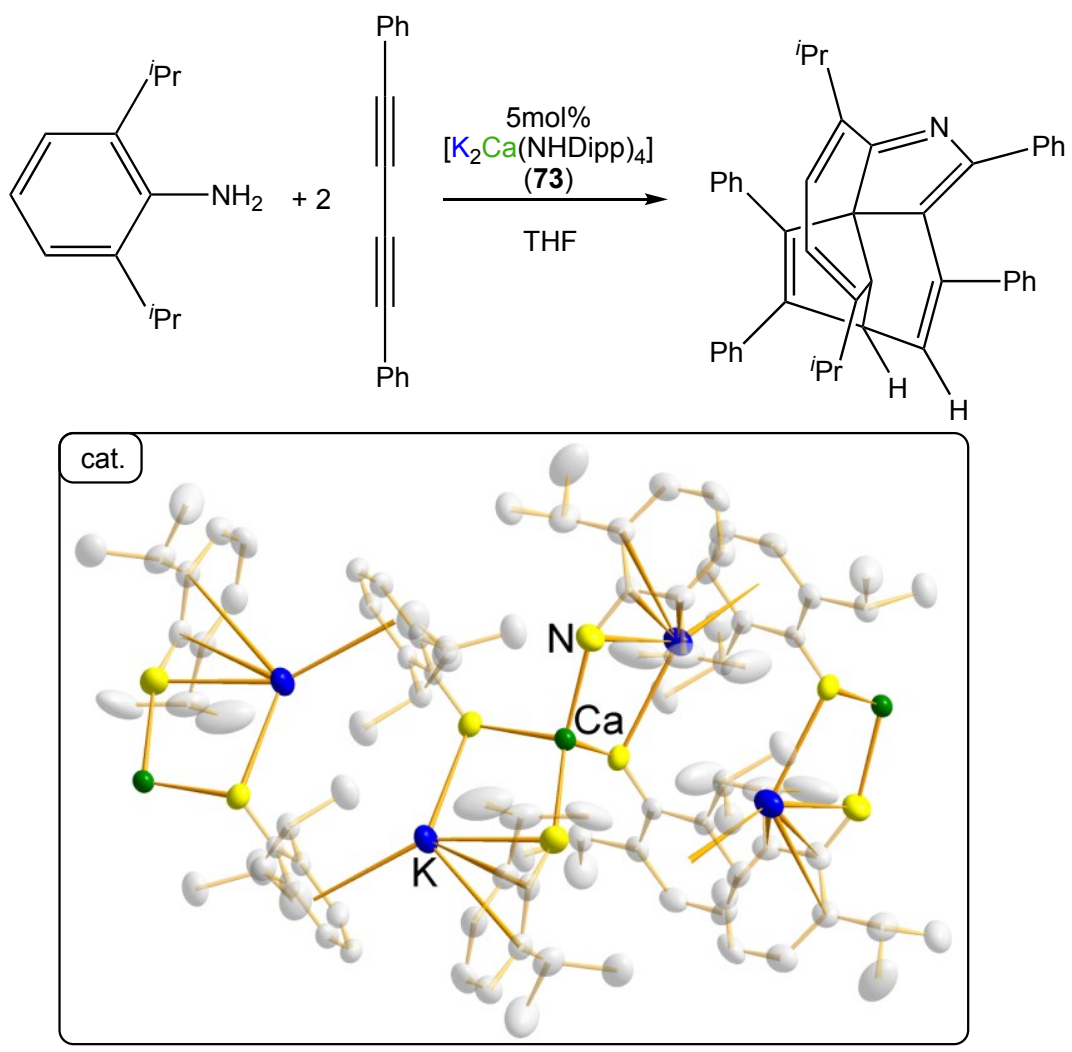
Hoping to promote enhanced reactivity by forming a calciate as seen earlier in calcium-mediated hydroamination reactions of alkynes, the dimesitylphosphane oxide supported dipotassium tetrakis(dimesitylphosphinito)calciate $\left[\mathrm{K}_{2} \mathrm{Ca}\left\{\mathrm{OPMes}{ }_{2}\right\}_{4}\left\{\mathrm{Mes}_{2} \mathrm{P}(\mathrm{O}) \mathrm{H}\right\}\right](76)$ was prepared (Scheme 45$)$. However, tested as a precatalyst for the hydrophosphorylation of isocyanates, its monometallic potassium component $\left[(\mathrm{THF}) \mathrm{K}\left(\mathrm{OPMes}_{2}\right)\right]_{4}$ proved to be more efficient, while the monometallic calcium component $\left[(\mathrm{THF})_{4} \mathrm{Ca}\left(\mathrm{OPMes}_{2}\right)_{2}\right](\mathbf{7 4})$ displayed a similar reactivity to the calciate. It was surmised from these findings that the calciate complex does not dissociate into its monometallic potassium and calcium counterparts. ${ }^{84}$

Scheme 45. Preparation of THF-solvated bis-dimesitylphosphinitocalcium (74, LHS) and its dimesitylphosphane oxide solvated dipotassium calciate derivative (76, RHS).

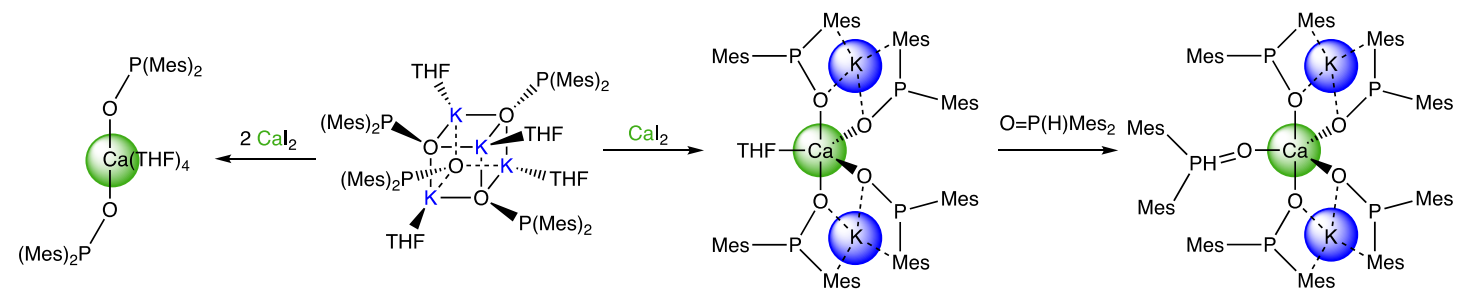

(74)

Cocomplexation has been employed to access a series of heterobimetallic allyl complexes. Potassium allyl, one and two equivalents, and bis(allyl)calcium have all been treated with bis(allyl)magnesium in THF to afford the lower order potassium tris(allyl)magnesiate $\left[\mathrm{KMg}\left(\mathrm{C}_{3} \mathrm{H}_{5}\right)_{3}(\mathrm{THF})_{n}\right]$ (77), the higher order dipotassium tetrakis(allyl)magnesiate $\left[\mathrm{K}_{2} \mathrm{Mg}\left(\mathrm{C}_{3} \mathrm{H}_{5}\right)_{4}\right](\mathbf{7 8})$ and the calcium tetrakis(allyl)magnesiate $\left[\mathrm{CaMg}\left(\mathrm{C}_{3} \mathrm{H}_{5}\right)_{4}\right](79)$, respectively (Scheme 46). ${ }^{85}$

Scheme 46. Heterobimetallic allyl complexes 77-79 prepared via cocomplexation. 


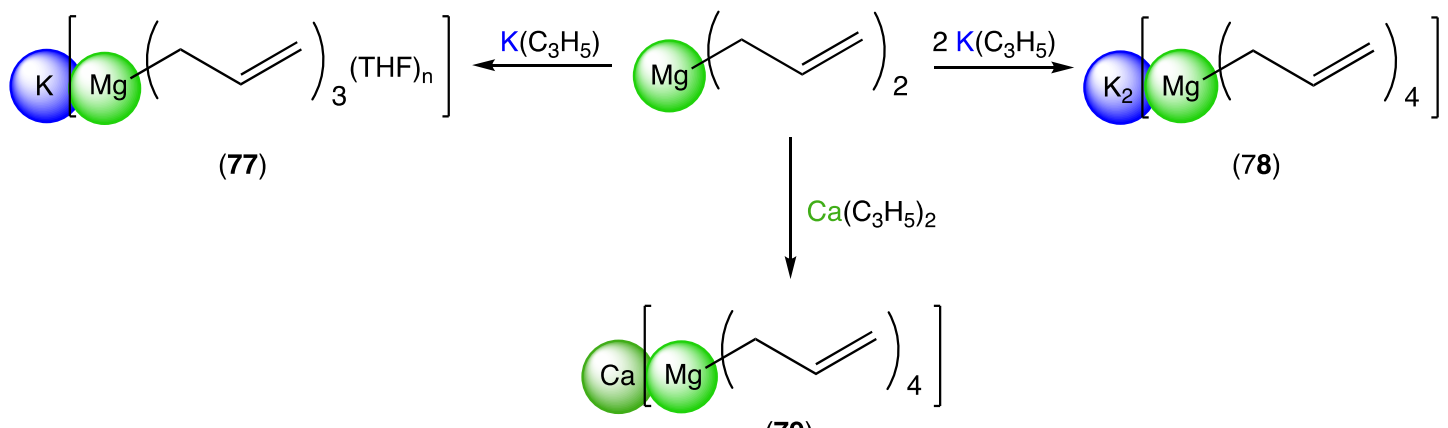

(79)

These three anionic heterobimetallic compounds initiate the polymerization of butadiene, giving polybutadiene (PBD) with low polydispersity indices below 1.10 and high 1 2-PDB contents up to $77 \%$. In contrast, in the absence of any bimetallic synergistic effects, the monometallic magnesium complexes cationic $\left[\mathrm{Mg}\left(\mathrm{C}_{3} \mathrm{H}_{5}\right)(\mathrm{THF})_{5}\right]\left[\mathrm{B}\left(\mathrm{C}_{6} \mathrm{~F}_{5}\right)_{4}\right]$ and neutral $\left[\mathrm{Mg}\left(\mathrm{C}_{3} \mathrm{H}_{5}\right)_{2}\right]$ fail to initiate, while the monometallic potassium component $\left[\mathrm{K}_{(}\left(\mathrm{C}_{3} \mathrm{H}_{5}\right)\right]$ did not initiate $\mathrm{BD}$ polymerization under standard conditions but required an increased catalyst loading to make PBD with a slightly increased PDI. Notable features included the rate of reaction being higher for dianionic than for the monoanionic potassium magnesiate; and that $\mathrm{K}^{+}$gave faster reaction rates than $\mathrm{Ca}^{2+}$ when employed as the counterion to the magnesiate moiety.

The homometallic azo complexes of lithium $\left[\mathrm{Li}_{2}\left\{\left(\eta^{2}-\mathrm{O}\left(\mathrm{C}_{10} \mathrm{H}_{6}\right) \mathrm{N}=\mathrm{N}\left(\mathrm{C}_{6} \mathrm{H}_{5}\right)\right\}_{2}\right]_{n}(80)\right.$ and magnesium $\left[\mathrm{Mg}\left\{\left(\eta^{2}-\mathrm{O}\left(\mathrm{C}_{10} \mathrm{H}_{6}\right) \mathrm{N}=\mathrm{N}\left(\mathrm{C}_{6} \mathrm{H}_{5}\right)\right\}_{2}\right]_{\mathrm{n}} \quad\right.$ (81) and the heterobimetallic lithium-magnesium derivative $\left[\mathrm{Li}_{2} \mathrm{Mg}_{2}\left\{\left(\eta^{2}-\mathrm{O}\left(\mathrm{C}_{10} \mathrm{H}_{6}\right) \mathrm{N}=\mathrm{N}\left(\mathrm{C}_{6} \mathrm{H}_{5}\right)\right\}_{6}\right]\right.$ (82) have been synthesized and characterized (Figure 19). A noticeable synergistic effect relates to solubility, as both homometallic compounds are insoluble in toluene; whereas the heterobimetallic composite dissolves in benzene in which it maintains its solid-state 
structure as determined from ${ }^{1} \mathrm{H}$-DOSY NMR experiments in $\mathrm{d}_{6}$-benzene solution. ${ }^{86}$ This structure has been interpreted as two truncated cubes that share a $(\mathrm{MgO})_{2}$ ring as one cube face. This family of compounds have been studied in rac-lactide polymerization. Measured activities were found to be variable, with the mixed heterometallic compound showing an activity intermediate between that of the higher active lithium and lower active magnesium homometallic compounds.
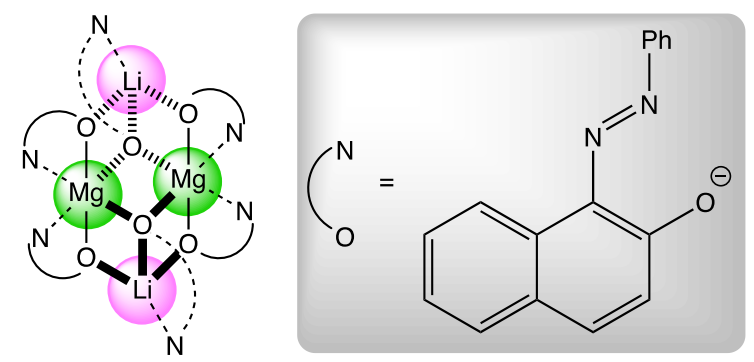

(82)

Figure 19. Molecular structure of the substituted lithium-magnesium azo complex $\left[\mathrm{Li}_{2} \mathrm{Mg}_{2}\left\{\left(\eta^{2}-\mathrm{O}\left(\mathrm{C}_{10} \mathrm{H}_{6}\right) \mathrm{N}=\mathrm{N}\left(\mathrm{C}_{6} \mathrm{H}_{5}\right)\right\}_{6}\right], 82\right.$

A study has been carried out incorporating a variety of chiral ligands into lithium butylmagnesiates, as reagents for asymmetric addition to aldehydes and ketones. ${ }^{87-89}$ Putative magnesiate species were prepared by ${ }^{n}$ BuLi deprotonation of a diol $\left(\mathrm{LH}_{2}\right)$ followed by the addition of either ${ }^{n} \mathrm{BuMgCl}$ or ${ }^{n} \mathrm{Bu}_{2} \mathrm{Mg}$ to access (L)BuMgLi or (L)Bu $\mathrm{BgLi}_{2}$, respectively (83 and $\mathbf{8 4}$, Scheme 47 ). Used in situ without isolation, these species were then treated with 2-bromopyridine and aromatic aldehydes in a stepwise manner to obtain pyridyl carbinols. Extensive optimisation studies have revealed that butyl magnesiates undergo $\mathrm{Mg}-\mathrm{Br}$ exchange efficiently at room temperature, but that only $(R, R)$-TADDOL or $(R)$-BIPHEN H2 were capable of inducing moderately asymmetric addition, affording opposite enantiomers. In order to achieve 
the best enantiomeric ratio, the trapping temperature had to be lowered to $-100{ }^{\circ} \mathrm{C}$ at the expense of overall yield, and an aromatic aldehyde with an electron-donating group such as 4-methoxybenzaldehyde had to be used, as the mixture would revert to racemic if an electron-withdrawing group were used. Subsequently this chemistry was extended to iodopyrazine.

Scheme 47. Preparation of lithium alkylmagnesiate complexes 83 and 84 incorporating chiral ligands derived from diols.

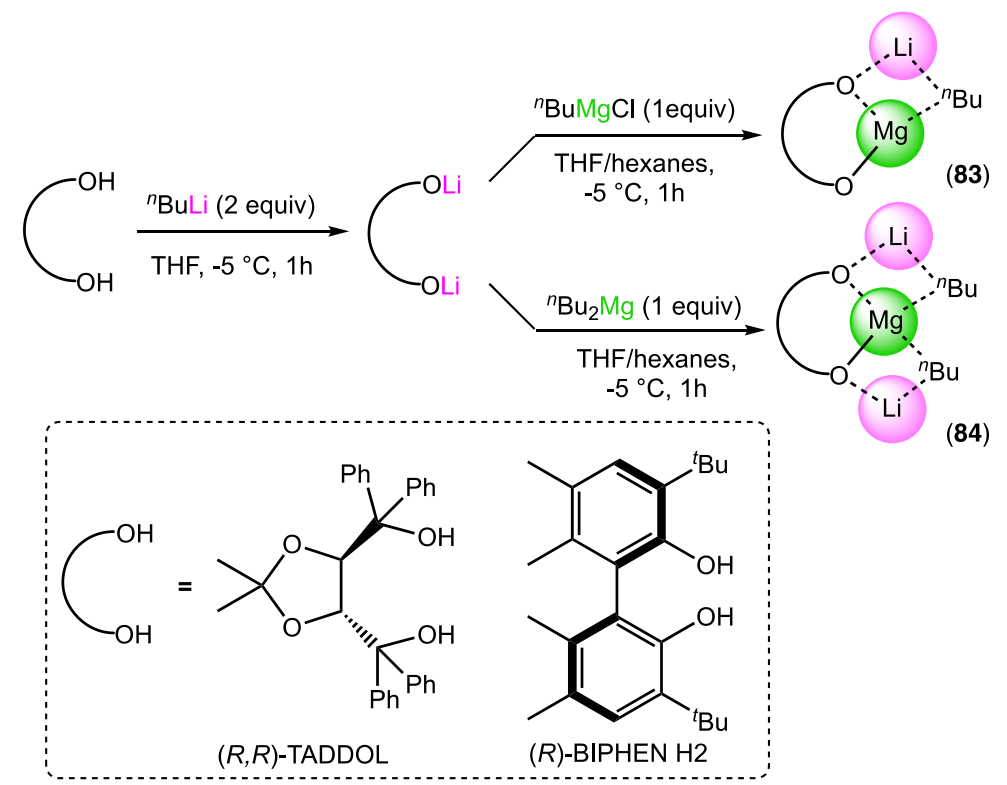

This work was extended to include other organoanions bound to the metals by altering the organomagnesium reagent utilized in the cocomplexation reaction. Having the general formula $(\mathrm{THF})_{n} \cdot \mathrm{Li}_{2} \mathrm{Mg}\{\mathrm{BIPHEN}\} \mathrm{R}_{2}$, (where $\mathrm{R}={ }^{n} \mathrm{Bu}, 85 ; \mathrm{Me}_{3} \mathrm{SiCH}_{2}, 86$; ${ }^{t} \mathrm{BuCH}_{2}, 87$ ), in the crystal these complexes exhibit tetrahedral $\mathrm{Mg}$ centers bound by 4 anions (2 $\mathrm{O}$ and $2 \mathrm{C}$ ), with 3- or 4-coordinate Li centers (made up of anionic and donor ligands) depending on the number of bound THF ligands. ${ }^{90}$ In terms of reactivity, a low temperature $\left(-60^{\circ} \mathrm{C}\right)$ reaction between 86 and 2-bromopyridine afforded the 
organometallic intermediate $(\mathrm{THF})_{2} \cdot \mathrm{Li} 2 \mathrm{Mg}\{(\mathrm{rac})-\mathrm{BIPHEN}\}\left(2-\mathrm{NC}_{5} \mathrm{H}_{4}\right)_{2}(\mathbf{8 8})$ in an isolated yield of $66 \%$ (Scheme 48$)$.

Scheme 48. Formation of $(\mathrm{THF})_{2} \cdot \mathrm{Li}_{2} \mathrm{Mg}\{(\mathrm{rac})-\mathrm{BIPHEN}\}\left(2-\mathrm{NC}_{5} \mathrm{H}_{4}\right)_{2}(\mathbf{8 8})$ made via metalhalogen exchange.

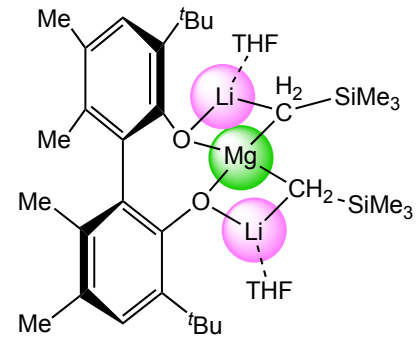

(86)
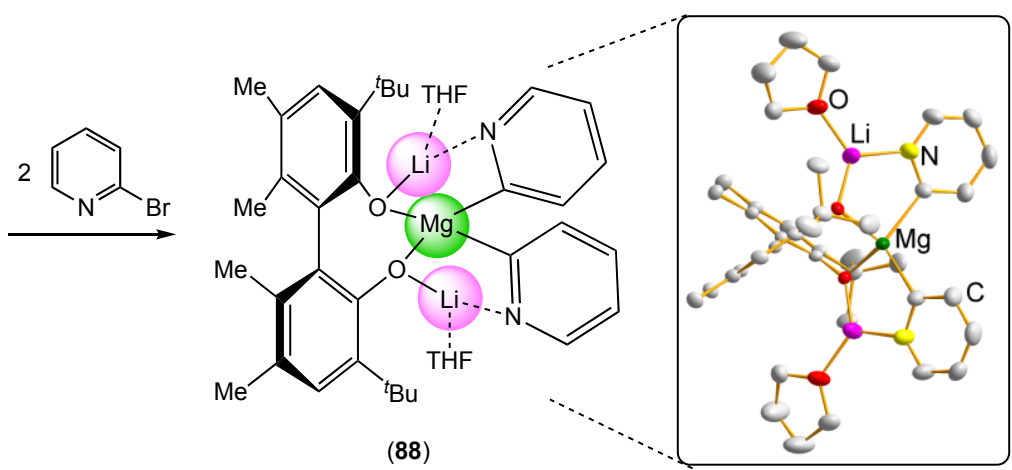

(88)

89 , was

converted to the solvent-free lithium alkylmagnesiate derivative $\left[\left\{\mathrm{Bu}_{2} \mathrm{Mg}\left\{\mathrm{LiOC}\left(\mathrm{CH}_{2}\right)_{5} \mathrm{CH}_{2} \mathrm{~N}(\mathrm{Me}) \mathrm{CH}_{2} \mathrm{CH}_{2} \mathrm{NMe}_{2}\right\}\right\}_{2}\right] 90$ upon reaction with $n$-butyllithium. Accordingly, treatment of this lithium alkylmagnesiate with a degassed $1.0 \mathrm{M}$ solution of water in diethyl ether afforded [ $\left.\left\{{ }^{n} \mathrm{BuMgOLi}\left[\mathrm{LiOC}\left(\mathrm{CH}_{2}\right)_{5} \mathrm{CH}_{2} \mathrm{~N}(\mathrm{Me}) \mathrm{CH}_{2} \mathrm{CH}_{2} \mathrm{NMe}_{2}\right]_{2}\right\}_{2}\right]$, 91, where an oxide anion, from twofold deprotonated water, has inserted into a MgLi2 triangle with loss of two equivalents of butane (figure 20).
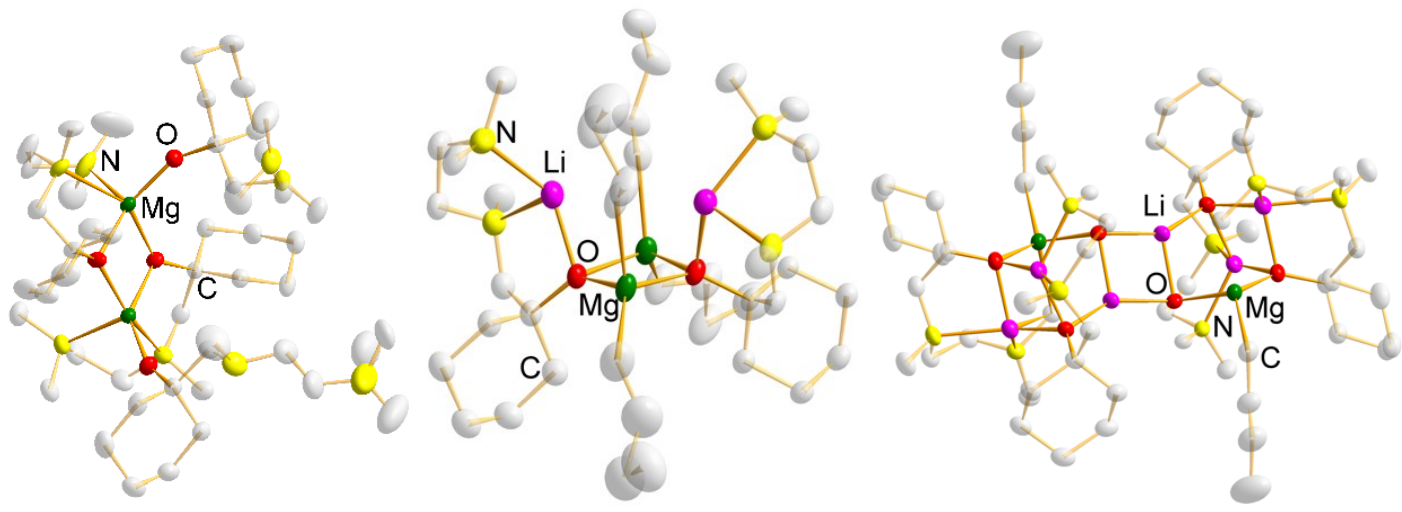
Figure 20. Molecular structures of the alkylmagnesium complex 89 and the lithium alkylmagnesiate complexes $\mathbf{9 0}$ and $\mathbf{9 1}$ all containing the same $\mathrm{N}, \mathrm{N}, \mathrm{O}$ tridentate ligand.

Complex 90 showed the best catalytic activities in Meerwein-Ponndorf-Verley (MPV) reduction applications, better than those of its homometallic magnesium precursor 89. For example, using benzaldehyde and dry isopropyl alcohol as a model reaction with 5 mol\% of catalyst in toluene, the benzyl alcohol product was formed in yields of $87.6 \%$ and $52.7 \%$, respectively (Scheme 49). Using 90 a range of carbonyl compounds (6 aldehydes and 2 ketones) were then selectively converted to the corresponding alcohols in yields ranging from 62.4 to $93.8 \% .{ }^{91}$ Further information is provided in the aluminum section 2.5.4.

Scheme 49. Catalyzed reduction of benzaldehyde by isopropyl alcohol.

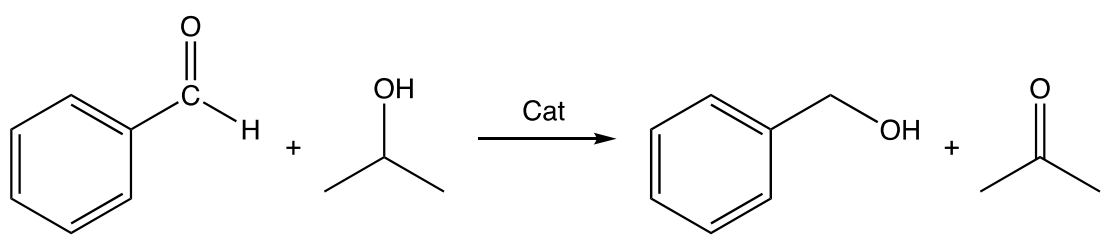

\subsubsection{Special Metathesis Reactions}

A recurring theme throughout this article is that on occasion straightforward looking metathesis reactions involving an organoalkali metal reagent and low polarity metal salts do not follow a straightforward path in practice. This complication has also been noted in a lithium-beryllium exchange reaction, a rarely studied type of metathesis. A reaction mixture of $n$-butyllithium, $N, N$-diethylhydroxylamine and beryllium chloride in a diethylether-THF mixture expected to give a simple beryllium bisdiethylhydroxylaminate product, in actuality produced a mixed lithium-beryllium 
diethylhydroxylaminate composition containing $n$-butylberyllium units of total molecular composition "Be(ONEt $)_{2}\left[\left\{\left(\text { LiONEt }_{2}\right)_{2}\left(n-B u-B^{2} \mathrm{ONEt}_{2}\right)\right\}_{2}\right]$ " (92). ${ }^{92} \quad$ This heptanuclear aggregate is made of two ladder-type subunits (comprising $\mathrm{Be}-\mathrm{O}, \mathrm{O}-\mathrm{Li}$, $\mathrm{Li}-\mathrm{O}$ and $\mathrm{O}-\mathrm{Be}$ rungs) connected in a beryllium-coordination tetrahedron of four oxygen centers (Figure 21). The authors of this work remark that "formation of such a complex reaction mixture might be attributed to a fine energetic balance between several species of very similar thermodynamic stability under the chosen reaction conditions".

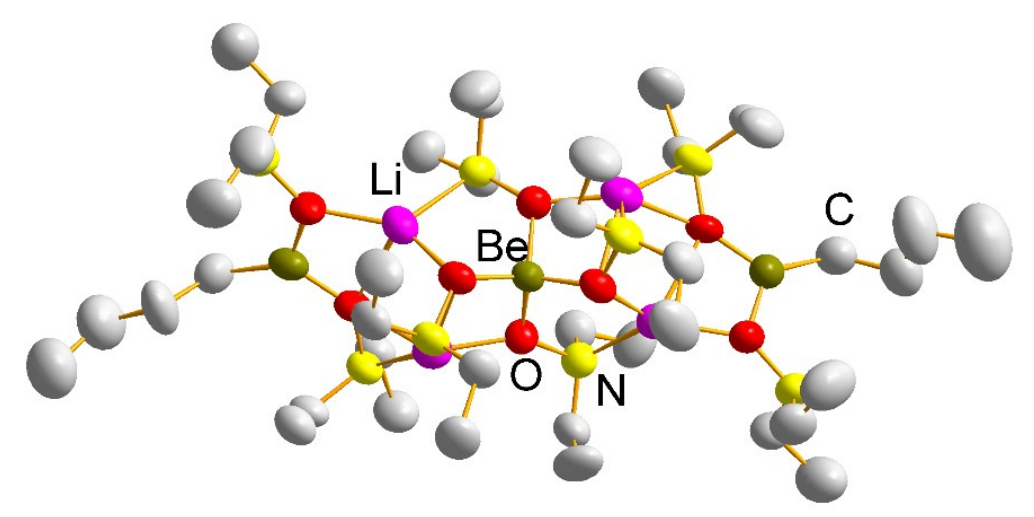

Figure 21. Molecular structure of the mixed lithium-beryllium complex $\left.\mathrm{Be}\left(\mathrm{ONEt}_{2}\right)_{2}\left[\left\{\left(\mathrm{LiONEt}_{2}\right)_{2}(\text { n-Bu-BeONEt})_{2}\right)\right\}_{2}\right]$ (92), a surprisingly complicated product from a simple metathesis reaction.

A "simple" metathesis reaction between KHMDS and a cyclic iminium chloride salt generating the free cyclic(alkyl)(amino)carbene (CAAC) ligand in situ, then introducing $\left[\left\{\mathrm{Sr}(\mathrm{HMDS})_{2}\right\}_{2}\right]$ in a 1:1:1 stoichiometric reaction overall was designed to produce $\left[\left(\left({ }^{R} C A A C\right) \operatorname{Sr}(H M D S)\right]\left(R=M_{2}\right)\right.$. However, this proved problematic as the product of repeated reactions was found instead to be the charge-separated strontiate complex $\left[\left\{\left({ }^{\mathrm{R}} \mathrm{CAAC}\right)_{3} \mathrm{~K}\right\}^{+}\left\{\mathrm{Sr}(\mathrm{HMDS})_{3}\right\}^{-}\right](93)$, though yields obtained were low (Scheme 50$) .{ }^{93}$ 
Scheme 50. Unexpected formation of the potassium strontiate product $\mathbf{9 3}$ from a "simple" halide metathesis reaction.

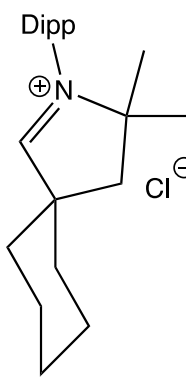

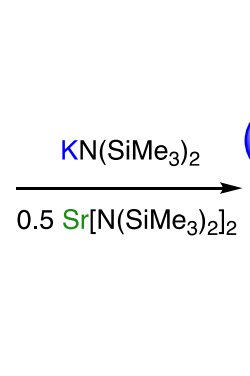

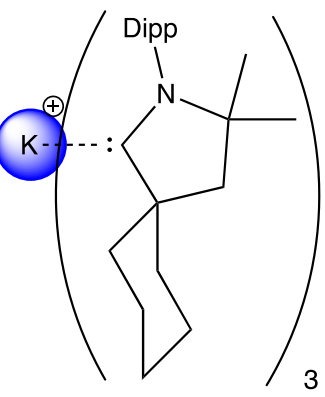

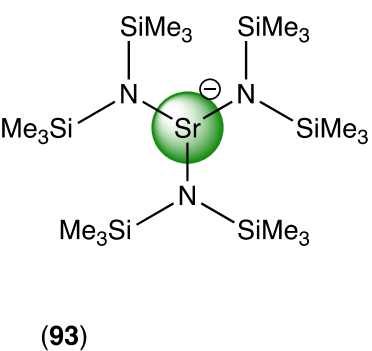

(93)

The importance of the identity of the alkali metal in metathesis reactions is brought home in the attempted synthesis of bis(2,6-diisopropoxyphenyl)calcium from treatment of 2,6-diisopropoxyphenylsodium with calcium iodide. Whereas this reaction gave an undefined mixture of products, switching to using the heavier alkali metal congener 2,6-diisopropoxyphenylpotassium afforded the calciate complex ([K] ${ }^{+}$ $\left.\left[\left\{\left({ }^{\mathrm{PrO}}\right)_{2} \mathrm{C}_{6} \mathrm{H}_{3}\right\}_{3} \mathrm{Ca}\right]^{-}\right)_{\infty}(\mathbf{9 4})$, which is reported to be the first arylcalciate complex of type $[\mathrm{M}]^{+}\left[\mathrm{Ar}_{3} \mathrm{Ca}\right]^{-}$to have been structurally characterized. It exits as a linear coordination polymer in the crystal (Figure 22). ${ }^{94}$ The authors make an important general point in this paper, namely "the exchange of different anions and cations may lead to complex mixtures of compounds in which certain combinations might be favored, or in the case that the product is obtained by crystallization, the least soluble species will be obtained." 


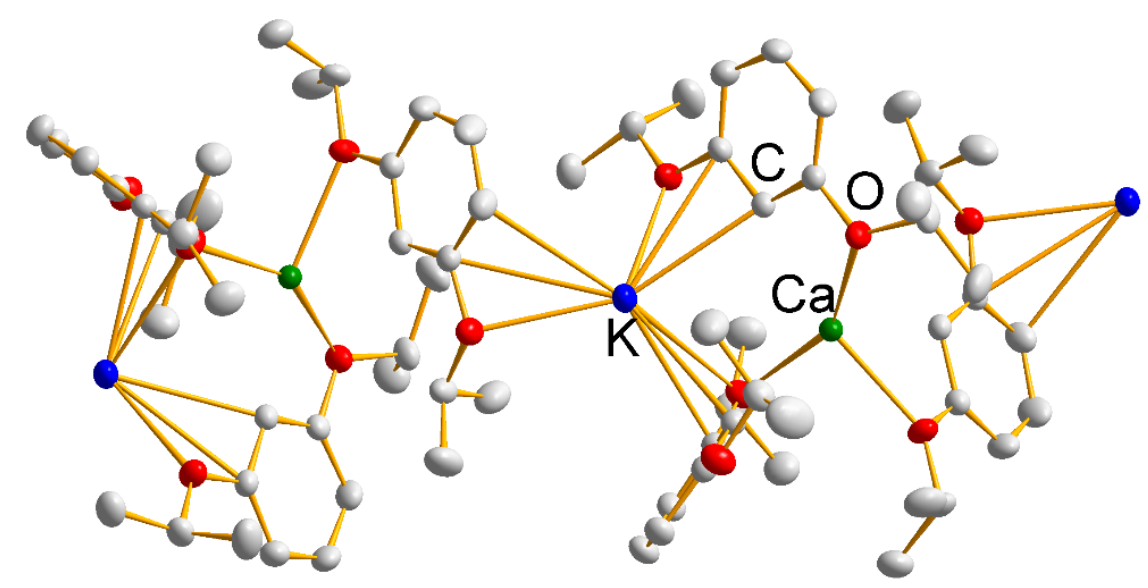

Figure 22. Part of the polymeric structure of the unusual potassium calciate complex $\left([\mathrm{K}]^{+}\left[\left\{\left({ }^{\mathrm{P} P O}\right)_{2} \mathrm{C}_{6} \mathrm{H}_{3}\right\}_{3} \mathrm{Ca}\right]^{-}\right)_{\infty}(94)$.

\subsubsection{Miscellaneous Complexes}

Alkali metal magnesiate complexes featuring the $\left[\mathrm{N}\left(\mathrm{SiMe}_{3}\right)\right.$ (Dipp)] ligand (where Dipp $\left.=2,6-{ }^{i} \mathrm{Pr}_{2}-\mathrm{C}_{6} \mathrm{H}_{3}\right)$ of formula $\left[\mathrm{MMg}\left\{\mathrm{N}\left(\mathrm{SiMe}_{3}\right)(\mathrm{Dipp})\right\}_{2}\left(\mu-{ }^{n} \mathrm{Bu}\right)\right](\mathrm{M}=\mathrm{Na}, 95$; or $\mathrm{K}, 96)$ have been synthesized by deprotonating two equivalents of the parent amine with equimolar amounts of the appropriate metal alkyl bases (Scheme 51). ${ }^{95}$

Scheme 51. Preparation of alkali metal magnesiate complexes 95 and 96 featuring the $\left[\mathrm{N}\left(\mathrm{SiMe}_{3}\right)(\mathrm{Dipp})\right]$ ligand and their breakdown to their monometallic constituents in $\mathrm{d}_{8^{-}}$ THF solution. 

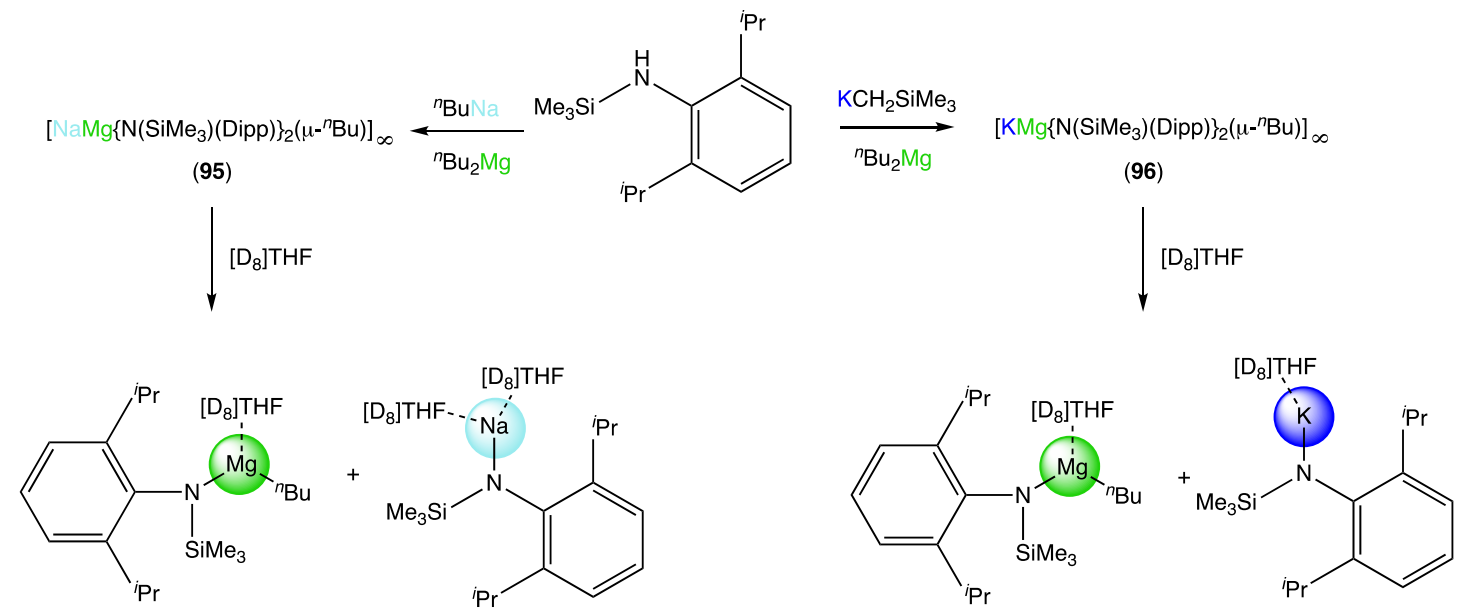

X-ray crystallographic studies determined that both complexes 95 and $\mathbf{9 6}$ form infinite helical chain arrangements with the $\mathrm{Mg}$ centers in $\sigma$-bonded trigonal planar $\mathrm{N}_{2} \mathrm{C}$ environments, while the softer $\mathrm{Na}$ and $\mathrm{K}$ centers engage in $\pi$-arene interactions with the Dipp groups (Figure 23). Prospects for any special synergistic reactivities were dashed by the insolubility of these polymers in arene solvents and breakdown to their monometallic components in THF- $\mathrm{C}_{6} \mathrm{D}_{6}$ mixtures as evidenced from NMR and DOSY experiments (scheme 51).
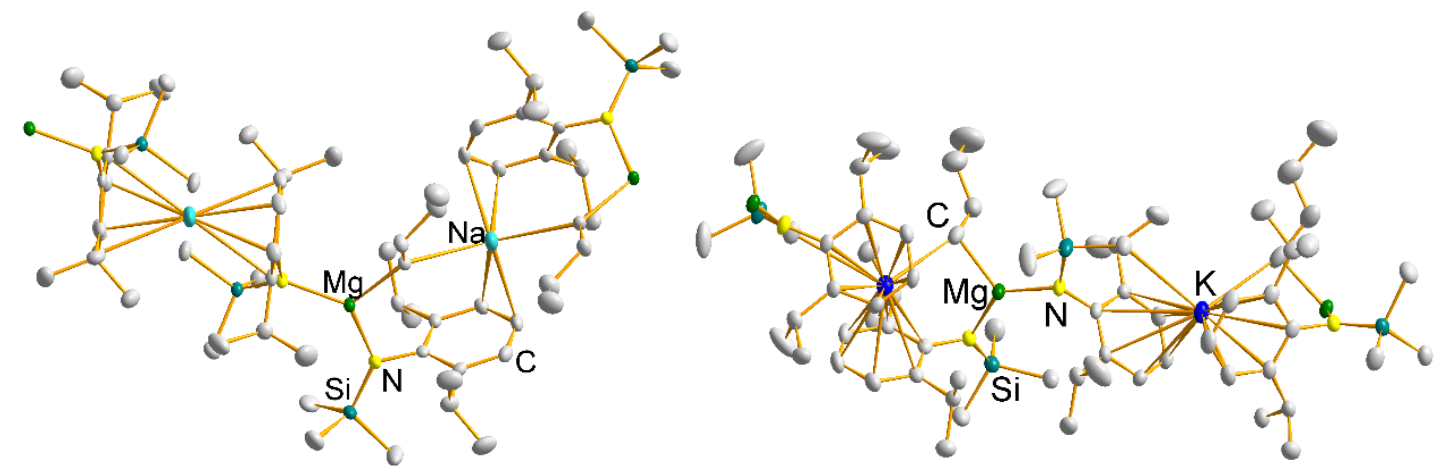

Figure 23. Polymeric structures of $\left[\mathrm{MMg}\left\{\mathrm{N}\left(\mathrm{SiMe}_{3}\right)(\operatorname{Dipp})\right\}_{2}\left(\mu-{ }^{n} \mathrm{Bu}\right)\right]$, where $\mathrm{M}=\mathrm{Na}$, 95; and $\mathrm{K}, 96$.

Heterobimetallic complexes are of interest as metal-organic chemical-vapor deposition (MOCVD) precursors having the advantage over their monometallic 
component species that they can facilitate the transport of two metals into the vapor phase while retaining control of the metal ${ }^{1}:$ metal $^{2}$ stoichiometry. Towards this purpose, a series of sodium $\left[\mathrm{NaMg}(\mathrm{PFTB})_{3}(\mathrm{THF})\right]_{3}(\mathbf{9 7})$, $\left[\mathrm{NaBa}(\mathrm{PFTB})_{3}(\mathrm{THF})\right]_{4}(\mathbf{9 8})$, and potassium $\quad\left[\mathrm{KMg}(\mathrm{PFTB})_{3}(\mathrm{THF})(\text { toluene })\right]_{\infty} \quad(\mathbf{9 9}), \quad\left[\mathrm{KSr}(\mathrm{PFTB})_{3}(\mathrm{THF})\right]_{4} \quad(\mathbf{1 0 0})$, $\left[\mathrm{KBa}(\mathrm{PFTB})_{3}(\mathrm{THF})\right]_{4}(\mathbf{1 0 1})$, alkaline-earth metal complexes complexes have been made by cocomplexing the Group 1 metal species with its Group 2 counterpart in THF (PFTB $=$ perfluoro-tert-butoxide). Thermal gravimetric analyses established that isostructural 97, 100 and 101 exhibit superior thermal properties compared to their Group 2 homometallic counterparts both in terms of volatility and residual weight percent. Their common structural motif places three bridging PFTB ligands between the two metal centers, with three terminal THF ligands on the octahedral Group 2 metals and one terminal THF ligand on the tetrahedral Group 1 metals (Figure 24). ${ }^{96}$ A feature of these structures is that metal-fluorine interactions are primarily associated with the alkali metal (highlighted in black in figure 24).

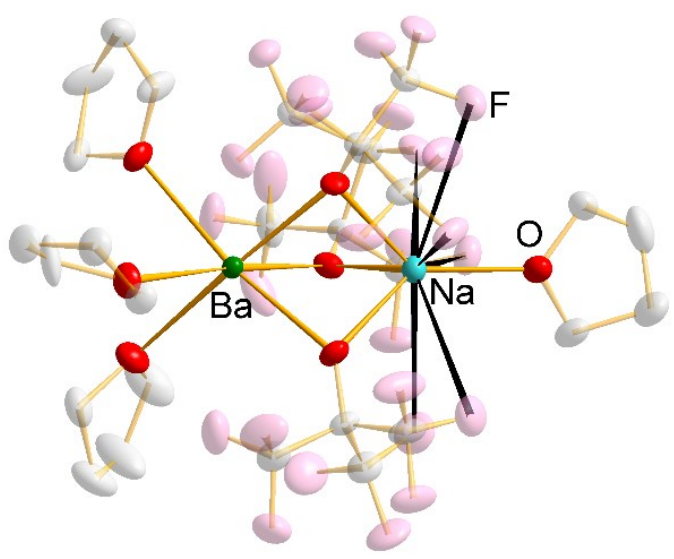

Figure 24. Molecular structure of the heterobimetallic perfluoro-tert-butoxide complex $\left[\mathrm{NaBa}(\mathrm{PFTB})_{3}(\mathrm{THF})\right]_{4}(\mathbf{9 8})$. 
Crystallographically characterized mixed s-block metal polymers are rare. An unprecedented amidinate-based example is the potassium-barium $N, N^{\prime}$-diisopropyl2-phenylethynylamidinate $\left[\left\{\mathrm{PhC} \equiv \mathrm{CC}\left(\mathrm{N}^{i} \mathrm{Pr}\right)_{2}\right\}_{3} \mathrm{BaK}\right]_{\infty}(\mathbf{1 0 2}$, Figure 25$)$.

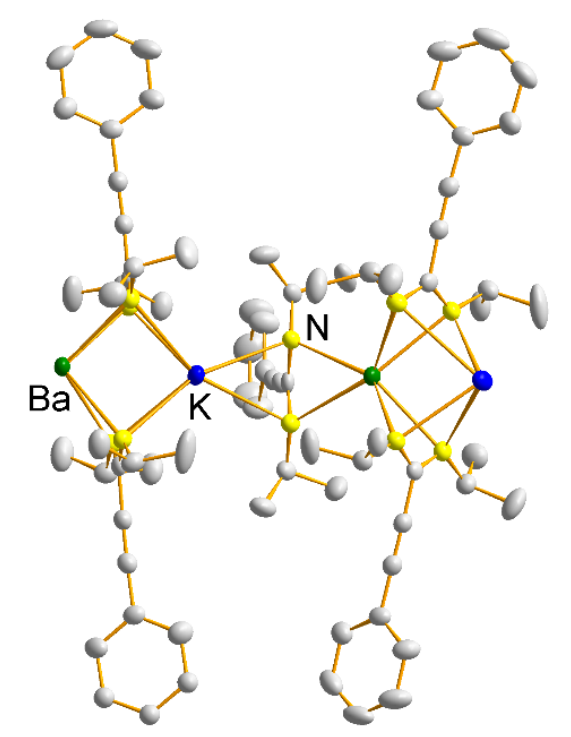

Figure 25. A section of the polymeric structure of the heterobimetallic mixed amidinato - amido complex $\left[\left\{\mathrm{PhC} \equiv \mathrm{CC}\left(\mathrm{N}^{\mathrm{P} P r}\right)_{2}\right\}_{3} \mathrm{BaK}\right]_{\infty}(\mathbf{1 0 2})$.

This was made fortuitously by introducing phenylacetylene and diisopropylcarbodiimide to a $\mathrm{d}_{6}$-benzene solution of " $\left[\mathrm{Ba}\left\{\mathrm{N}\left(\mathrm{SiMe}_{3}\right)_{2}\right\}_{2}\right]$ ". Further investigation found that this barium starting material was heavily contaminated with the $\left[\mathrm{BaK}\left\{\mathrm{N}\left(\mathrm{SiMe}_{3}\right)_{2}\right\}_{3}\right]$ "ate" complex (103). Other attempted reactions between barium iodide and KHMDS in THF with a stoichiometry designed to afford $\left[\mathrm{Ba}\left\{\mathrm{N}\left(\mathrm{SiMe}_{3}\right)_{2}\right\}_{2}(\mathrm{THF})_{2}\right]$, also produced 103 . This again demonstrates the complexity that can often creep into "simple" metathesis reactions, with in this case cocomplexation seemingly taking place between the desired $\mathrm{Ba}(\mathrm{HMDS})_{2}$ product and KHMDS starting material (scheme 52). ${ }^{97}$ 
Scheme 52. Impure metathesis reaction producing neutral and anionic barium species.

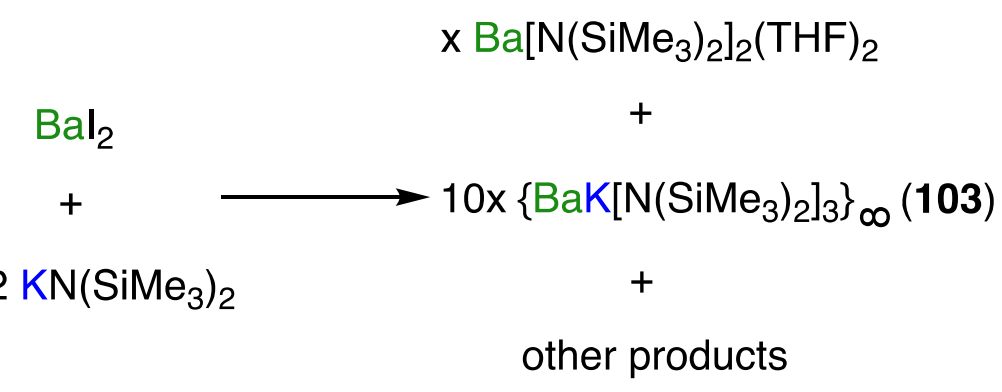

\subsection{Turbo-Grignard Reagent and Related Salt-Supported Complex Chemistry}

\subsubsection{Introduction}

In terms of utility in organic synthesis, the synergistic reagents that have made by far and away the greatest impact in recent times are the so-called "turbo-reagents", some examples of which have been patented..$^{98}$ In general, these come in two distinct sets: those that perform metal-hydrogen exchange; and those that perform metal-halogen exchange. Most important in the former category are bases of magnesium (TMPMgCl-LiCl, 104; $\left.\mathrm{TMP}_{2} \mathrm{Mg} \cdot 2 \mathrm{LiCl}, \quad 105\right)$ and zinc (TMPZnCl.LiCl, 106; $\left.\mathrm{TMP}_{2} \mathrm{Zn} \cdot 2 \mathrm{MgCl}_{2} \cdot 2 \mathrm{LiCl}, 107\right)$. The magnesium bases can be regarded as synergistic cocomplexes between Hauser bases $\left(R_{2} N M g X\right)$ or bis-amides $\left[\left(R_{2} N\right)_{2} M g\right]$ with molecules of lithium chloride. The molecule or molecules of lithium chloride are thought to increase the solubility of the magnesium (and zinc) TMP component, though their presence is also likely to increase the polarity of the magnesium (and zinc) component, in a combination of factors that leads to enhanced basicity compared to the lithium chloride free compounds. These "turbo-charged" reagents are outstanding for selectively deprotonating a myriad of aromatic and heterocyclic substrates usually performed in THF solution to generate organometallic intermediates that have the capacity to react directly with a wide assortment of 
electrophiles that makes them invaluable within numerous synthetic campaigns. Knochel and co-workers, who have pioneered these bases, have elaborated their reactivity in a comprehensive review, ${ }^{99}$ while a most recent short review entitled "polyfunctional zinc and magnesium organometallics for organic synthesis: some perspectives" has also appeared. ${ }^{100}$ For specific detail on the exceedingly broad scope of the organic chemistry taking place in these reactions readers should refer to these reviews and the primary literature associated with them. To avoid unnecessary repetition, here, following introductory examples to demonstrate the general reactivity characteristics and scope of these bases, the focus will be on their more recent applications with emphasis on the reaction types that synergistic influences are at work and the different substrates involved rather than on reviewing each different electrophilic trapping or cross coupling method used for onward reactivity. The

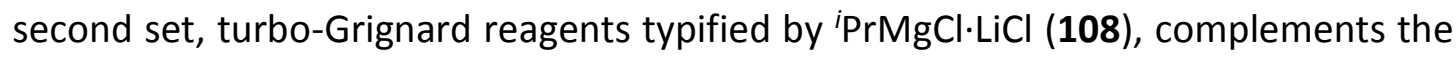
aforementioned base set as they are effective for metal-halogen exchange reactions. These lithium-chloride supported Grignard reagents exhibit significantly enhanced rates of $\mathrm{Mg}-\mathrm{Br}$ and $\mathrm{Mg}-\mathrm{I}$ exchange in reactions with aromatic and heteroaromatic compounds, compared to those in the absence of lithium chloride. Knochel and coworkers have also just recently reviewed these turbo-Grignard reagents, ${ }^{101}$ so to avoid unnecessary repetition here illustrative examples will be given to show their versatility with emphasis on more recent developments, including cases where the halide components are replaced by bulky alkoxide components that aid solubility.

\subsubsection{Turbo Reagents in Metal-Hydrogen Exchange: Background}


The mono-amide turbo reagent TMPMgCl-LiCl (104) is easily accessed by action of the salt-supported Grignard reagent ${ }^{i} \mathrm{PrMgCl} \cdot \mathrm{LiCl}(\mathbf{1 0 8})$ on the parent amine $\mathrm{TMP}(\mathrm{H})$ in THF after 24 hours at ambient temperature. ${ }^{102}$ Isolated crystalline samples of $\mathbf{1 0 4}$ have been found to adopt a dinuclear monomeric structure with the one-coordinate TMP ligand terminally attached to magnesium, which forms a $\mathrm{Mg}(\mu-\mathrm{Cl})_{2} \mathrm{Li}$ bridge (Scheme 53)..$^{103}$

Scheme 53. Preparation of Turbo-Hauser reagents TMPMgCl.LiCl (104), $(\mathrm{TMP})_{2} \mathrm{Mg} \cdot 2 \mathrm{LiCl}$ (105), $\mathrm{TMP}_{2} \mathrm{Zn} \cdot 2 \mathrm{MgCl}_{2} \cdot 2 \mathrm{LiCl}$ (107) and $\mathrm{TMPZnCl} \cdot \mathrm{LiCl}$ (106) and molecular structure of $\mathbf{1 0 4}$ (as its tri-THF solvate: inset).

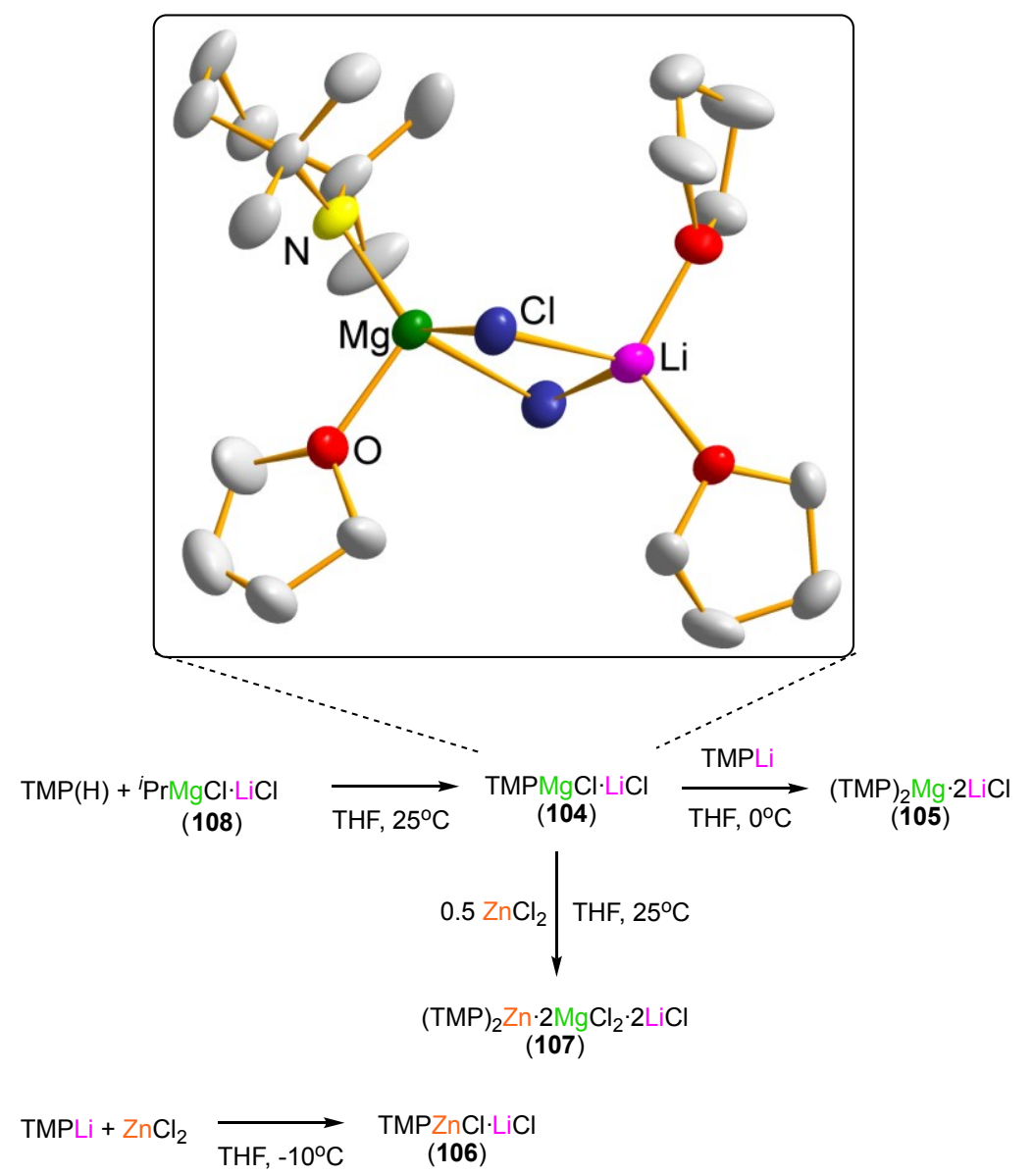


High kinetic activity of the molecular TMPMgCl$\cdot \mathrm{LiCl}$ composition is evident from its magnesiation of isoquinoline to give 2-quinolylmagnesium chloride compared to that of the diisopropylamide analog ${ }^{i} \mathrm{Pr}_{2} \mathrm{NMgCl} \cdot \mathrm{LiCl}(109$, Scheme 54).

Scheme 54. Comparative magnesiation studies of isoquinoline using 104 and 109.

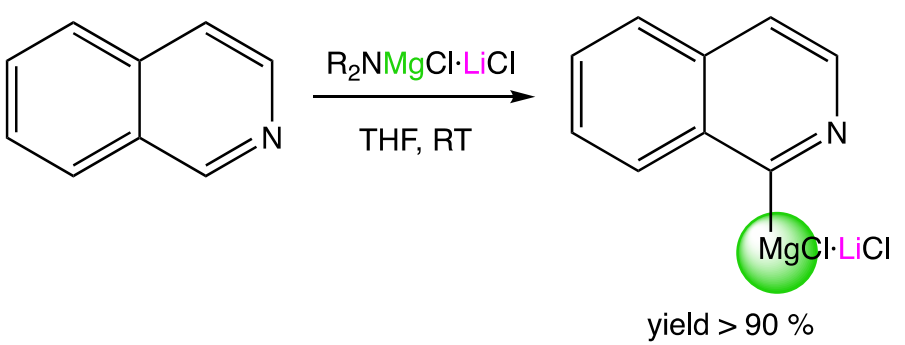

\begin{tabular}{|c|c|c|}
\hline $\mathbf{R}_{\mathbf{2}} \mathbf{N}$ & equiv & time \\
\hline${ }^{i} \operatorname{Pr}_{2} \mathrm{~N}(\mathbf{1 0 9})$ & 2.0 & $12 \mathrm{~h}$ \\
\hline $\operatorname{TMP}(\mathbf{1 0 4})$ & 1.1 & $2 \mathrm{~h}$ \\
\hline
\end{tabular}

Functional group tolerance enhanced from that of conventional organolithium bases is often a characteristic feature of the metalation reactions of TMPMgCl$\cdot \mathrm{LiCl}$, reflecting the reduced polarity of the $\mathrm{Mg}-\mathrm{C}$ bonds against that of $\mathrm{Li}-\mathrm{C}$ bonds, which generally exhibit much lower functional group tolerance. Even substrates having as many as four sensitive substituents as in the tetrasubstituted benzene [containing two esters, a $t$-butoxycarbonyloxy, and a benzoyl] (Scheme 55), can be readily deprotonated in full by stoichiometric TMPMgCl$\cdot \mathrm{LiCl}$ (at $-20^{\circ} \mathrm{C}$ within $2 \mathrm{~h}$ ) before undergoing acylation and having the Boc substituent removed using $\mathrm{CF}_{3} \mathrm{CO}_{2} \mathrm{H}$, to form the penta-substituted phenol (Scheme 55). ${ }^{104}$

Scheme 55. Utilization of a turbo-base in the preparation of a penta-substituted phenol starting from a phenol with groups usually sensitive to metalation.<smiles>CCOC(=O)c1cc(OC(C)(C)C)c(C(=O)c2ccccc2)c(C(=O)OCC)c1</smiles><smiles>C[C@H](O)[C@H](C)Cl</smiles><smiles>CCOC(=O)c1cc(C(=O)OCC)c(C(=O)c2ccccc2)c(OC(C)(C)C)c1C(=O)OCC</smiles>

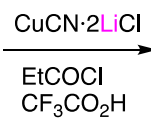<smiles>CCOC(=O)c1cc(C(=O)OCC)c(C(=O)c2ccccc2)c(O)c1C(=O)OCC</smiles> 
Impressively, all positions of a benzene ring can be functionalized as demonstrated by the conversion of ethyl 3-chlorobenzoate to a hexa-substituted benzene product, where the turbo base TMPMgCl-LiCl performs a regioselective metalation at the four intermediate stages of the synthesis (Scheme 56). ${ }^{104}$

Scheme 56. Transformation of ethyl 3-chlorobenzoate to a fully (hexa) substituted benzene product involving multiple turbo-base metalations.<smiles>CCOC(=O)c1cccc(Cl)c1</smiles>

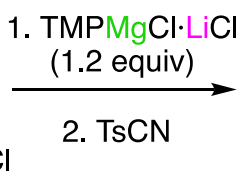<smiles>CCOC(=O)c1cccc(Cl)c1C#N</smiles>

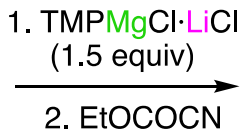<smiles>CCOC(=O)c1ccc(Cl)c(C#N)c1C(=O)OCC</smiles>

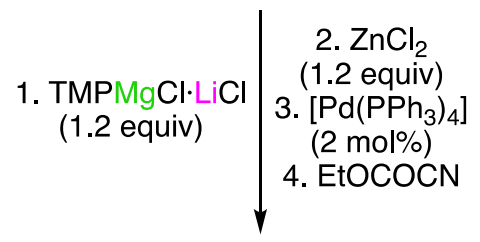<smiles>CCOC(=O)c1c(C#N)c(Cl)c(C#N)c(C(=O)OCC)c1C(=O)OCC</smiles>

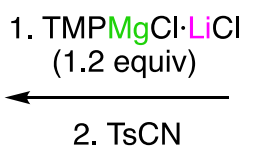<smiles>CCOC(=O)c1cc(Cl)c(C#N)c(C(=O)OCC)c1C(=O)OCC</smiles>

The reach of this turbo base is impressive, for example extending to metallocenes and a host of challenging heterocycles including thiophenes, furans, pyridines, quinolines, pyrazoles, pyrimidines, etc. Electron-deficient nitrogen-based heterocyclic scaffolds are prevalent in the pharmaceutical industry so the ability to efficiently regioselectively metalate them is often crucial in one or more steps in many synthetic campaigns. This is challenging especially if more than one nitrogen or other heteroatom resides in the ring since this will increase the electrophilicity of the 
metalated heterocyclic intermediates and make them susceptible to competitive addition reactions leading to dimerization. ${ }^{105} \mathrm{TMPMgCl} \cdot \mathrm{LiCl}$ can be extremely effective in meeting such challenges as can be seen in the representative example of converting 2-bromopyrimidine to a fully functionalized pyrazolopyrimidine in a multi-step process where metalation takes place at the 4,6 , and 5 positions consecutively (Scheme 57). ${ }^{106,107}$

Scheme 57. Transformation of 2-bromopyrimidine to a pyrazolopyrimidine functionalized in all positions involving multiple metalation steps.<smiles>Brc1ncccn1</smiles>

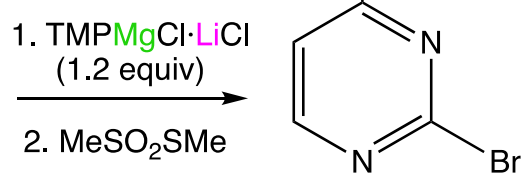

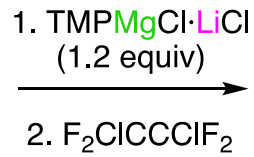<smiles>CSc1cc(Cl)nc(Br)n1</smiles>

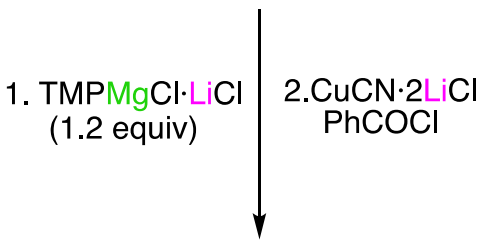<smiles>CS(=O)(=O)c1nc(Br)nc2[nH]nc(-c3ccccc3)c12</smiles><smiles>CS(=O)(=O)c1nc(Br)nc(Cl)c1C(=O)c1ccccc1</smiles>

Where TMPMgCl$\cdot \mathrm{LiCl}$ fails to metalate or is slow to metalate a substrate at low temperature, for example, an aromatic compound decorated with electron donating groups or weakly electron-withdrawing groups, the stronger deprotonating agent $\mathrm{TMP}_{2} \mathrm{Mg} \cdot 2 \mathrm{LiCl}(\mathbf{1 0 5})$, made by adding an equivalent of LiTMP to TMPMgCl$\cdot \mathrm{LiCl}$ in THF solution, can be employed (Scheme 53 ). This bis-TMP base takes only $10 \mathrm{~min}$ at $-40^{\circ} \mathrm{C}$ to magnesiate dimethyl-1,3-benzodioxan-4-one at the ortho-position adjacent to the 
$\mathrm{O}(\mathrm{C}=0)$ function (Scheme 58), ${ }^{108}$ while its powerful basicity also enables the magnesiation of a range of substituted aromatic rings using the phosphorodiamidate group $\left[\left(\mathrm{Me}_{2} \mathrm{~N}\right)_{2} \mathrm{P}(\mathrm{O}) \mathrm{O}-\right]$ for directing the metalation. ${ }^{109}$

Scheme 58. Selective ortho-metalation of dimethyl-1,3-benzodioxan-4-one followed by transmetalation with $\mathrm{ZnCl}_{2}$, Pd-catalyzed cross-coupling with $(E)$-1-hexenyl iodide, hydrogenation of double bond and dioxanone cleavage.<smiles>CC1(C)OC(=O)c2ccccc2O1</smiles>

The turbo class of deprotonating reagents was extended to zinc to increase further the functional group tolerance. This covers common hypersensitive groups such as esters and nitriles as well as aryl ketone substrates, which in general are incompatible with the aforementioned turbo magnesium bases. The mono-amide zinc reagent $\mathrm{TMPZnCl} \cdot \mathrm{LiCl}(\mathbf{1 0 6})$ is prepared by a seemingly simple cocomplexation reaction between LiTMP and zinc chloride at $-10{ }^{\circ} \mathrm{C}$ in THF. ${ }^{110}$ (Scheme 59, top). But this reaction can also be likened to the trans-metal-trapping (TMT, see section 2.5.3) process as in typical salt metathesis reactions (scheme 59, bottom) the lithium reactant (e.g., an organolithium compound) normally exchanges with a zinc halide (here $\mathrm{ZnCl}_{2}$ ) to generate the lithium halide and the organozinc halide or bis(organo)zinc derivative depending on stoichiometry, but here the exchange stops at a crossover point furnishing the turbo-zinc reagent which contains both lithium and zinc. 
Scheme 59. Top: preparation of the turbo-zinc reagent TMPZnCl.LiCl (106); bottom: analogy with a trans-metal-trapping reaction involving an organoaluminum reagent.
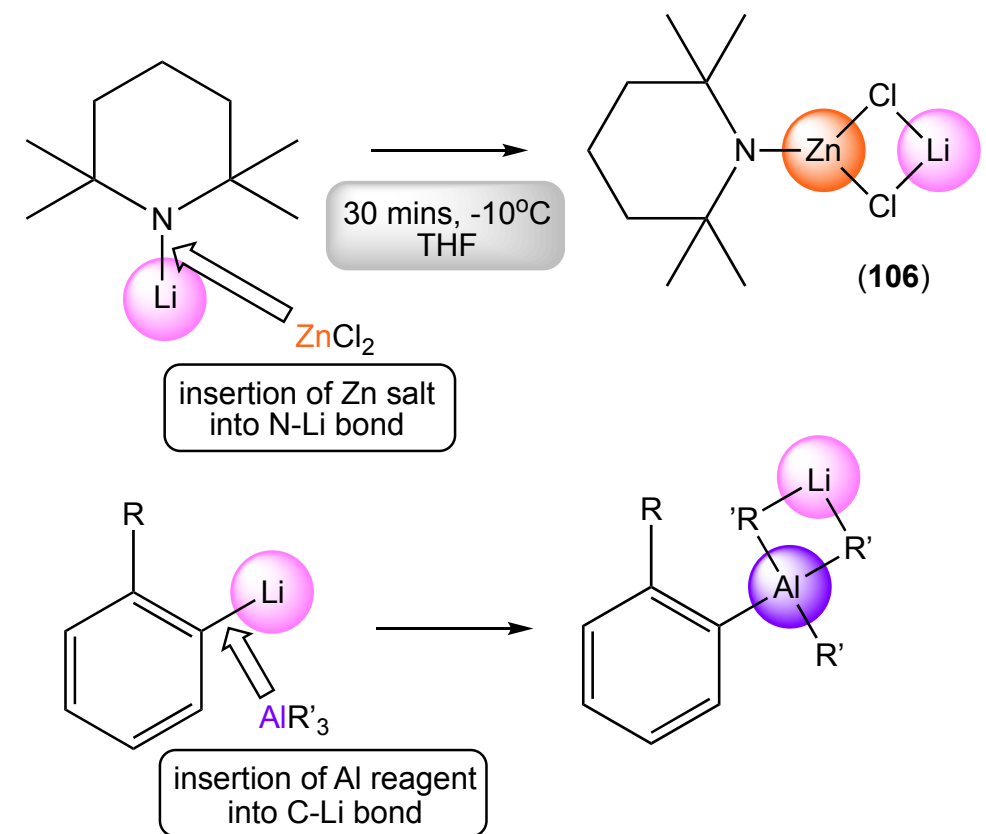

Note, however, that no crystallographic structures of any turbo-zinc reagent have been reported to date. The advantages of $\mathrm{TMPZnCl} \cdot \mathrm{LiCl}$ include high stability at ambient temperature under an anaerobic atmosphere, generally no requirement to perform $\mathrm{C}-\mathrm{H}$ to $\mathrm{C}-\mathrm{Zn}$ reactions at subambient temperatures, and the ability to be utilized at elevated temperatures. The last point is emphasized in the reaction with 3,6-dimethoxypyridazine. This represents a challenging substrate to metalate at a reasonable rate because of its electron-donating substituents, but under microwave irradiation ( 1 hour at $90^{\circ} \mathrm{C}$ ) TMPZnCl$\cdot \mathrm{LiCl}$ can achieve complete zincation to generate a zincated intermediate that can then undergo a Negishi cross-coupling to form the pyridazine product (Scheme 60). ${ }^{111}$

Scheme 60. Turbo-metalation of 3,6-dimethoxypyridazine and its subsequent functionalization. 


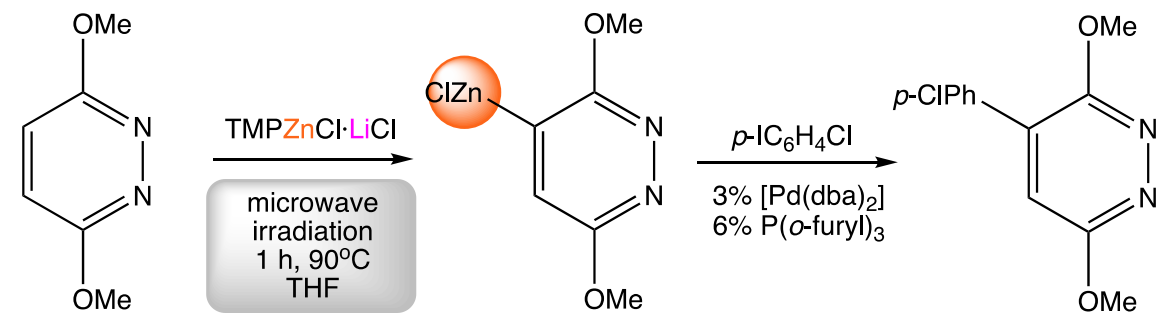

Doubling the TMP stoichiometry by cocomplexing TMPMgCl$\cdot \mathrm{LiCl}$ with zinc chloride in a 2:1 ratio in THF solution generates a stronger deprotonating turbo-zinc reagent with the novel heterotrimetallic formula $\mathrm{TMP}_{2} \mathrm{Zn} \cdot 2 \mathrm{MgCl}_{2} \cdot 2 \mathrm{LiCl}$ (107, scheme 53). Hypersensitive multiheteroatom cyclic substrates that with conventional bases have a propensity towards ring opening under metalation conditions can be metalated with $\mathrm{TMP}_{2} \mathrm{Zn} \cdot 2 \mathrm{MgCl}_{2} \cdot 2 \mathrm{LiCl}$ with the ring intact. This is observed with conversion of a 1,2,4triazole to an allylic substituted variant in a reaction where both TMP ligands are active in the metalation (Scheme 61). ${ }^{112}$

Scheme 61. Conversion of a 1,2,4-triazole to an allylic variant using turbo-metalation.<smiles>[Pb]n1cncn1</smiles>

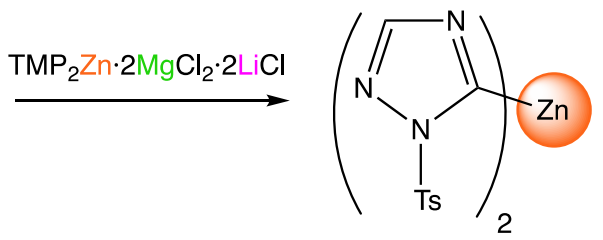<smiles></smiles><smiles>C=CCc1ncnn1P</smiles>

In specific syntheses where the substrate to be deprotonated contains a Lewis basic group or atom these turbo-magnesium and turbo-zinc bases can be modified by adding another Lewis acid (commonly boron trifluoride). This addition can change the regioselectivity of a reaction. 3-Fluoropyridine provides an illustrative example. In the absence of $\mathrm{BF}_{3}\left(\mathrm{OEt}_{2}\right)$, TMPMgCl-LiCl executes magnesiation at the 2 position of the heterocycle, which allows onward functionalization at that position to give a 2,3disubstituted pyridine product; whereas when $\mathrm{BF}_{3}\left(\mathrm{OEt}_{2}\right)$ is present it sterically blocks 
the 2-position by coordinating to the $\mathrm{N}$ and thus the same reaction sequence produces the 3,4-disubstituted pyridine product (Scheme 62). ${ }^{113} \mathrm{~A}$ range of similar TMP bases activated by lithium chloride have been made including the aluminum reagents $\mathrm{TMP}_{3} \mathrm{Al} \cdot 3 \mathrm{LiCl}(\mathbf{1 1 0})$ and $\left[{ }^{t} \mathrm{Bu}\left({ }^{\mathrm{i} P r}\right) \mathrm{CH}\left({ }^{t} \mathrm{Bu}\right) \mathrm{N}\right]_{3} \mathrm{Al} \cdot 3 \mathrm{LiCl}(\mathbf{1 1 1})$ but to date these have not shown anything like the utility of their magnesium and zinc counterparts. ${ }^{114}$

Scheme 62. Regioselectivity changes on performing turbo-metalation in the absence and presence of boron trifluoride.
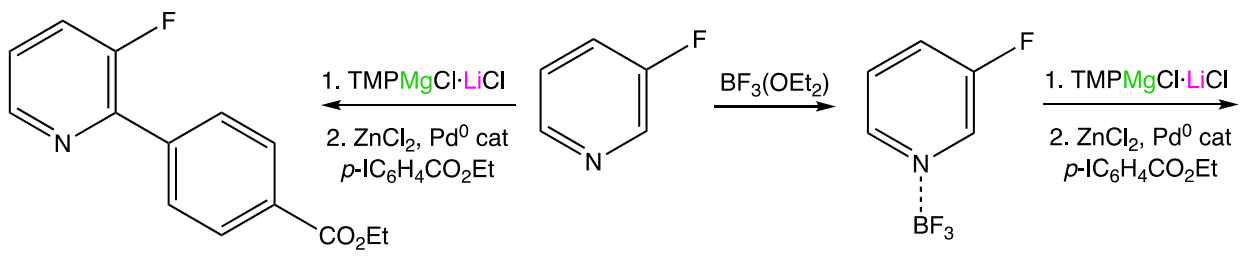<smiles>CCOC(=O)c1ccc(-c2ccncc2F)cc1</smiles>

\subsubsection{Turbo Metal-Hydrogen Exchange Reactions: Recent Advances}

Tetrasubstituted allenes have been prepared by using two successive metalation/Pdcatalyzed coupling steps where the base employed is TMPZnCl $\mathrm{LiCl}(\mathbf{1 0 6})$, starting from 1-(trimethylsilyl)-3-aryl-1-propyne precursors. ${ }^{115} 106$ has also been used to metalate 4,4-dimethyloxazoline to generate a stable 2-zincated oxazolinyl reagent. ${ }^{116}$ This study was stimulated by the fact that oxazoline units are well known in synthetic campaigns because of strong directing abilities in directed ortho-metalation (DoM) reactions, while they belong to a versatile class of heterocycle that can possess a long list of useful properties including antibiotic, antifungal, antitumor, cytotoxic or neuroprotective ones. ${ }^{117}$ 2-Lithio oxazolines prepared by action of a conventional organolithium base are prone to ring fragmentation leading to isonitriles; whereas 
these 2-zincated oxazolines with more covalent $\mathrm{M}-\mathrm{C}$ bonds remain intact at $25^{\circ} \mathrm{C}$ and thus are amenable to arylation via Negishi cross-couplings with an assortment of aryl iodides and bromides (Scheme 63).

Scheme 63. Comparison between turbo-metalation and conventional lithiation of oxazoline, giving ring-intact and ring-open products respectively.

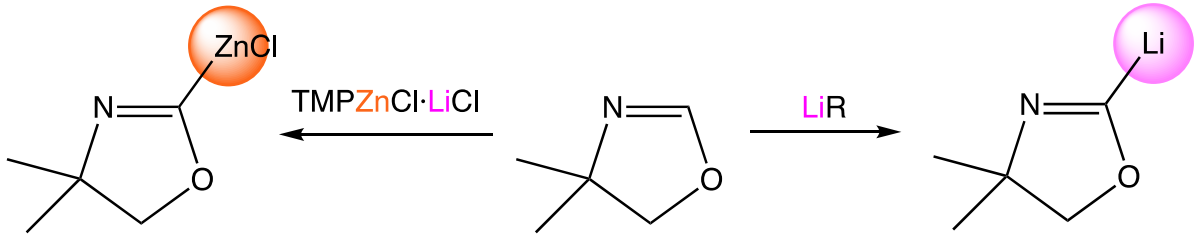

$$
\downarrow E^{+}
$$

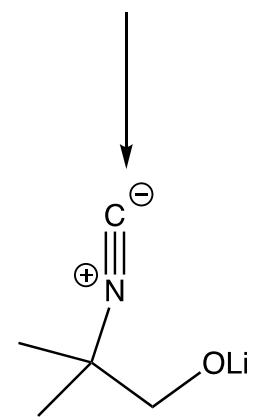

Scheme 64 shows an example of such an arylation that once taken place can direct an ortho-magnesiation (using 104) through its oxazolinyl group, followed by transmetalation with $\mathrm{ZnCl}_{2}$ and Pd-catalyzed cross-coupling, to form a biaryl that also undergoes magnesiation (using 105), which after transmetalation using $\mathrm{CuCN} \cdot 2 \mathrm{LiCl}$ and addition of allyl bromide gives the desired oxazoline.

Scheme 64. Functionalization of oxazoline and its benzonitrile substituent using three turbo-metalation steps. 

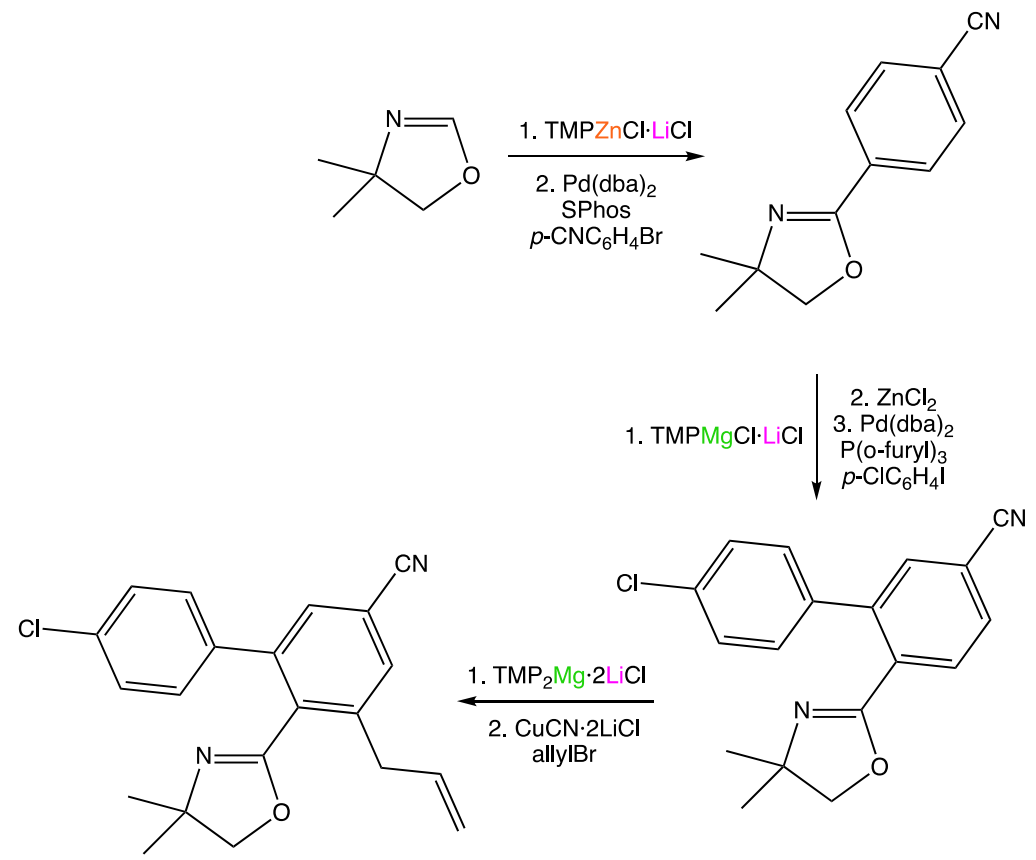

Of importance in biological chemistry, the tropolone scaffold features in over 200 natural products. Tropolone derivatives can also be easily zincated using the mild turbo-base TMPZnCl.LiCl. ${ }^{118}$ This feat is beyond the ability of the stronger bases LDA, LiTMP or even TMPMgCl-LiCl that cause various protected tropolones to fragment even at subambient temperatures; whereas the zincated intermediates can be easily intercepted by a variety of electrophiles or by in situ Negishi cross-coupling as shown for 1-fluoro-4-iodobenzene (Scheme 65).

Scheme 65 . Selective turbo-zincation of a tropolone followed by interception with the electrophile 1-fluoro-4-iodobenzene.
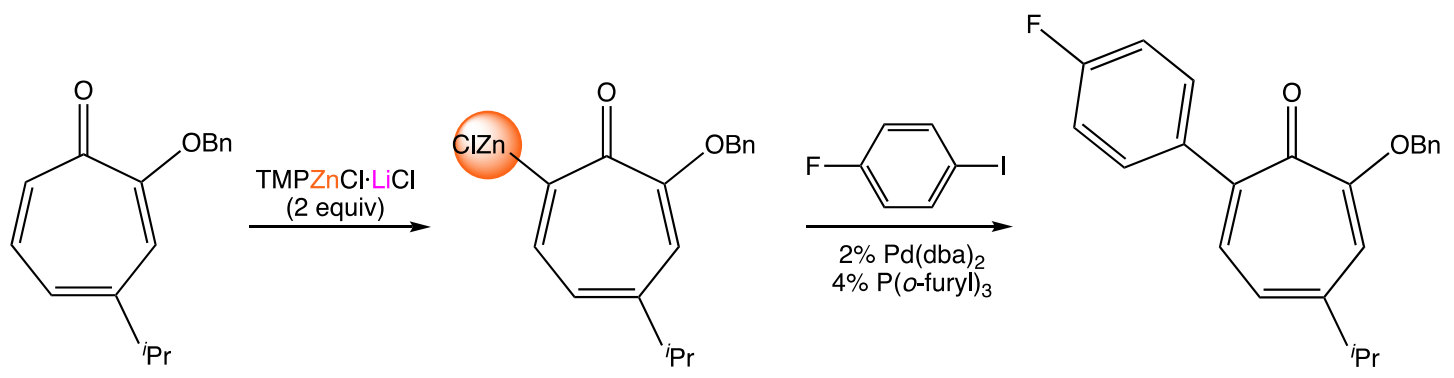
Three component $\mathrm{TMP}_{2} \mathrm{Zn} \cdot 2 \mathrm{MgCl} 2 \cdot 2 \mathrm{LiCl}$ has also been utilized for the regioselective zincation of methoxyethoxymethyl (MEM)-protected 2-pyridone and 2,7naphthyridone scaffolds. ${ }^{119}$ Zincations of these scaffolds followed by electrophilic interception afford functionalized 2-pyridones and 2,7-naphthyridones (Scheme 66). Follow-up magnesiation could be accomplished using a combination of magnesiumiodine exchange by ${ }^{i} \mathrm{PrMgCl} \cdot \mathrm{LiCl}$ and deprotonation using TMPMgCl$\cdot \mathrm{LiCl}$.

Scheme 66. Turbo-zincation of a pyridone and quenching with an electrophile.

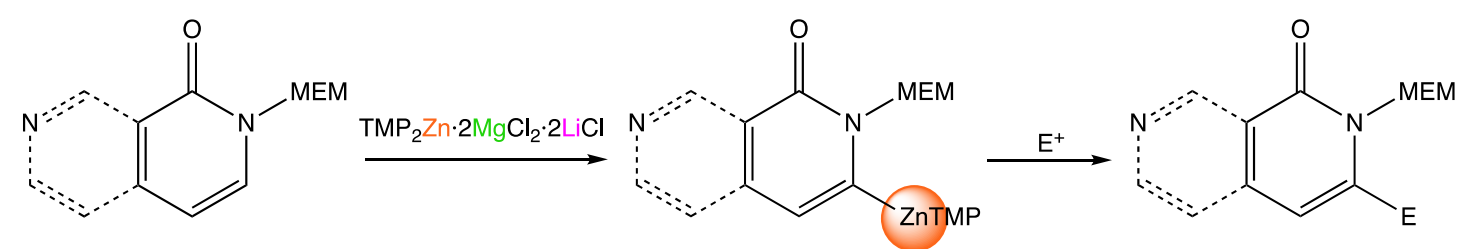

Evidence that TMPZnCl$\cdot \mathrm{LiCl}$ zincations can be upscaled in practical processes has been demonstrated with chromones, which are naturally occurring heterocycles that display useful pharmaceutical characteristics, spanning antibacterial, antineoplastic and anti-HIV activities. ${ }^{120}$ Turbo-zincations have been performed on a $50 \mathrm{mmol}$ scale and usually occur at the C3 position of the heterocycle. However, in the presence of $\mathrm{MgCl}_{2}$ the regioselectivity switches to the $\mathrm{C} 2$ position. In the latter synergistic case the Lewis acidity of the salt blocks the $\mathrm{C} 3$ position by binding to the adjacent carbonyl function (scheme 67).

Scheme 67. Regioselectivity change on turbo-zincation of chromone on the addition of magnesium chloride. 
<smiles>O=c1ccoc2ccccc12</smiles>

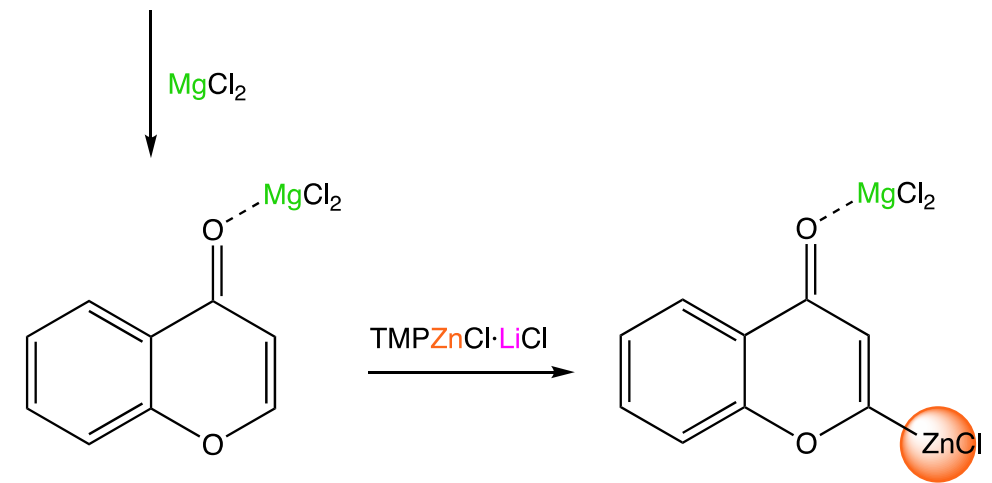

This regioselective control of metalation reactions by Lewis acidic $\mathrm{MgCl}_{2}$ has also been documented with uracils and uridines. ${ }^{121}$ When the salt is not present, TMPMgCl$\cdot \mathrm{LiCl}$ magnesiation of uracil and uridine derivatives occurs at position $\mathrm{C}(5)$; whereas in its presence, zincation by $\mathrm{TMP}_{2} \mathrm{Zn} \cdot 2 \mathrm{MgCl}_{2} \cdot 2 \mathrm{LiCl}$ switches to position $\mathrm{C}(6)$ (scheme 68).

Scheme 68. Comparison of regioselectivity in turbo-zincation and turbo-magnesiation reactions of a uracil.

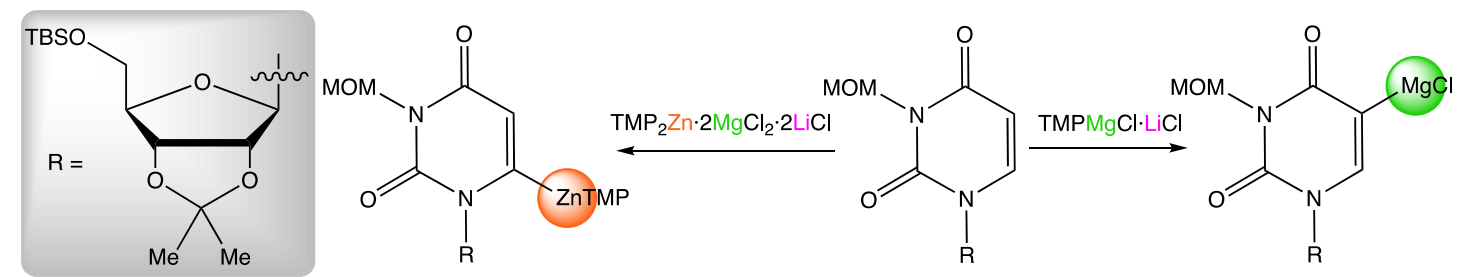

Smooth turbo-zincation and turbo-magnesiation reactions of 1,4-dithiin derivatives have also been reported by applying $\mathrm{TMPZnCl} \cdot \mathrm{LiCl}$ and $\mathrm{TMPMgCl} \cdot \mathrm{LiCl}$ respectively. 1,4-Dithiins are important synthetic building blocks that belong to the sulfur heterocyclic family. ${ }^{122}$ Following trapping by electrophiles, the resulting functionalized 1,4-dithiins can be metalated a second time by the same turbo bases to afford 2,3disubstituted-1,4-dithiins after a second electrophilic interception (scheme 69). 
Scheme 69. Mono- and di-functionalization of 1,4-dithiins using turbo-bases.<smiles>CCC(C)C1=CSC(F)=CS1</smiles>

Both $\mathrm{TMP}_{2} \mathrm{Zn} \cdot 2 \mathrm{MgCl}_{2} \cdot 2 \mathrm{LiCl}$ and TMPMgCl$\cdot \mathrm{LiCl}$ have been used to metalate (zincate or magnesiate respectively) polyfunctional silylated cyanohydrins. ${ }^{123}$ Metalated intermediates can then be trapped with different electrophiles including allylic and benzylic bromides, acid chlorides, aldehydes, $\mathrm{NCCO}_{2} \mathrm{Et}$ or $\mathrm{MeSO}_{2} \mathrm{SMe}$, while onward reactivity with TBAF (tetra- $n$-butylammonium fluoride)-deprotection produces keto or 1,2-diketo derivatives (Scheme 70).

Scheme 70. Lateral turbo-metalation of polyfunctional silylated cyanohydrins and their conversion to keto derivatives.

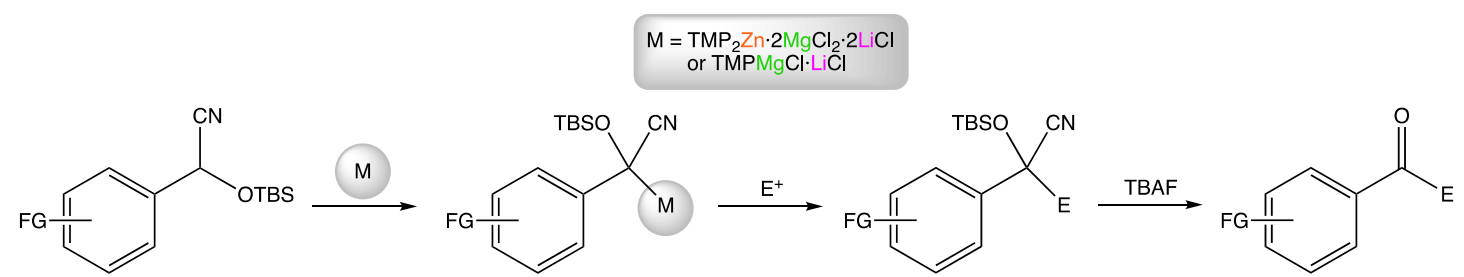

These same zinc and magnesium bases have been used for the one-, two-, three- and four-fold functionalization of tetrathiafulvalene (TTF). ${ }^{124}$ This follows four successive metalation/electrophilic-quenching steps, in reactions leading to acylation, allylation, arylation, halogenation or thiolation of the 5-membered rings (Scheme 71).

Scheme 71. Sequential functionalization of tetrathiafulvalene (TTF) to fully substituted derivatives using turbo-bases at each step. 

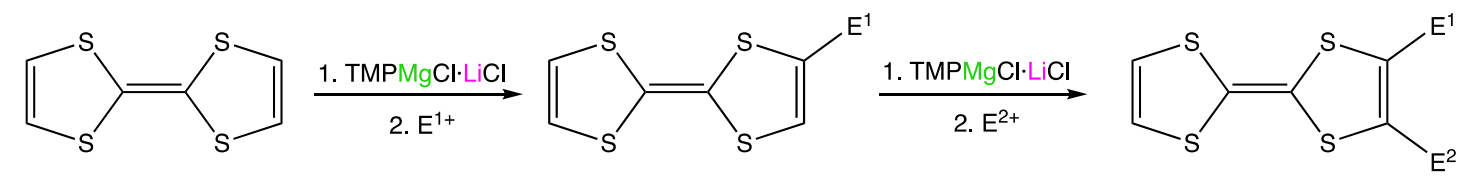

1. TMPZnCl.LiCl $\$ 2. $\mathrm{E}^{3+}$

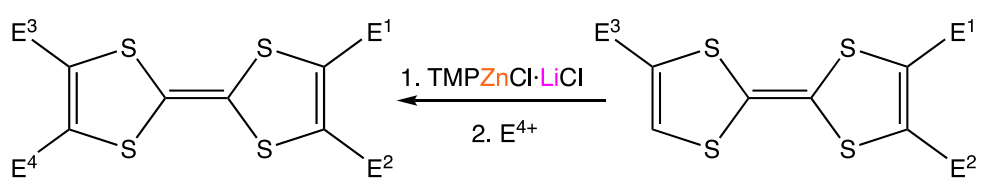

Such regioselective functionalizations are important, as the TTF scaffold is a key component of organic materials that can exhibit unique electronic, magnetic and optical properties. Fourfold functionalized tetrathiafulvalene derivatives can also be synthesized starting from 1,3-dithiole-2-thiones, where $\mathrm{TMPMgCl} \cdot \mathrm{LiCl}$ is used in two metalation/electrophilic-trapping steps to produce difunctionalized 1,3-dithiole-2thione derivatives, which in turn can undergo a triethylphosphite induced crosscoupling with their oxygen counterparts to generate new symmetrical and nonsymmetrical tetraaryl tetrathiafulvalenes (Scheme 72)..$^{125}$

Scheme 72. Transformation of 1,3-dithiole-2-thiones to tetraaryl tetrathiafulvalenes using a turbo-base approach. 
<smiles>S=c1sccs1</smiles>

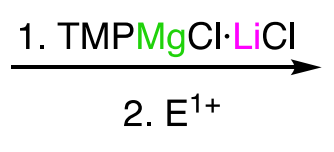<smiles>CCc1csc(=S)s1</smiles><smiles>[Z]c1sc(=O)sc1C</smiles><smiles>[Z7]C1=C([CH])SC(=C2SC([Z7])=C([As])S2)S1</smiles>
1. TMPMgCl·LiCl 2. $\mathrm{E}^{2+}$

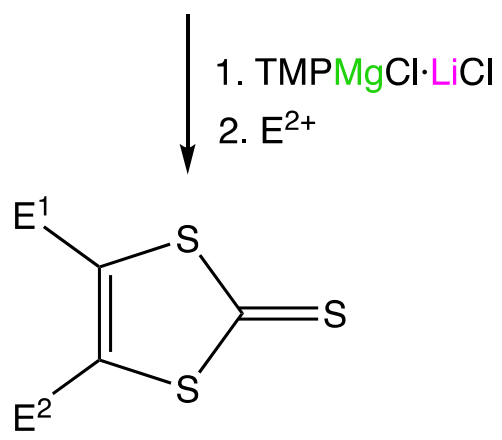

Through regioselective metalation these salt-supported TMP bases can open up different access points within the ring structure of 1,5-naphthyridine for electrophilic interception. The bis(amide) $\mathrm{TMP}_{2} \mathrm{Mg} \cdot 2 \mathrm{LiCl}$ magnesiates the parent 1,5-naphthyridine scaffold at $-78^{\circ} \mathrm{C}$ in THF at the C4-position to furnish 4-substituted 1,5-naphthyridines following quenching with various electrophiles (Scheme 73). ${ }^{126}$ In turn, 4-substituted 1,5-naphthyridines favor precomplexation of the mono-TMP magnesiate TMPMgCl$\cdot \mathrm{LiCl}$ or zinc counterpart $\mathrm{TMPZnCl} \cdot \mathrm{LiCl}$ at $\mathrm{N} 1$ to direct metalation (magnesiation or zincation) to $\mathrm{C} 8$, which can be accessed by electrophiles to afford 4,8-substituted 1,5-naphthyridines. 2,4-Disubstituted 1,5-naphthyridines can be obtained via magnesiation of 4-substituted 1,5-naphthyridines by $\mathrm{TMPMgCl} \cdot \mathrm{LiCl}$ provided the Lewis acid $\mathrm{BF}_{3} \cdot \mathrm{OEt}_{2}$ is added to the starting heterocycle to complex the N1 position. 2,4,8-Trifunctionalized and 2,4,7,8-tetrasubstituted 1,5-naphthyridines could also be synthesized in reactions involving halogen dance steps.

Scheme 73. Assorted functionalizations of the 1,5-naphthyridine scaffold actioned by sequential turbo-base/electrophilic interception protocols. 


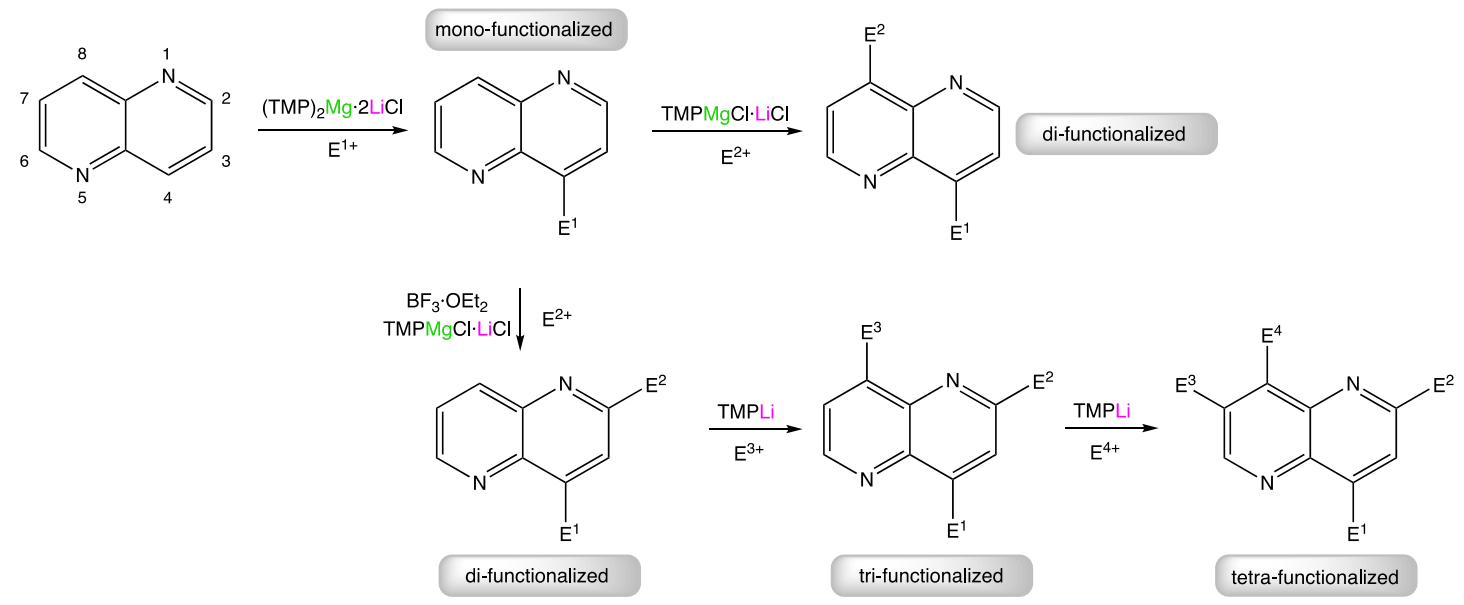

\subsubsection{Solid Air- and Moisture-Resistant Turbo-Zinc Pivalate-Containing Reagents}

A series of synergistic solid zinc allylic reagents have been accessed through reacting allylic chlorides or bromides with zinc dust in the company of lithium chloride and magnesium pivalate $\left[\mathrm{Mg}\left(\mathrm{OCO}^{t} \mathrm{Bu}\right)_{2}, \mathrm{Mg}(\mathrm{OPiv})_{2}\right]$ in THF solvent. The solvent is removed under vacuum to produce the desired solids. ${ }^{127}$ These reagents exhibit excellent reactivity in Pd-catalyzed cross-coupling reactions with aryl bromides when employing the PEPPSI-IPent catalyst. ${ }^{128}$ The synergistic reactivity behind these zinc allylic reagents is due to the presence of magnesium pivalate and lithium chloride in the reaction mixtures. Emphasizing this point, "[2-(ethoxycarbonyl)cyclohex-2-en-1yl]zinc pivalate" in dry THF can be cross-coupled with 2-bromotoluene in THF solution to form the product ethyl 2'-methyl-1,4,5,6-tetrahydro-[1,1'-biphenyl]-2-carboxylate in $79 \%$ yield (Scheme 74). In contrast, switching from the pivalate to the corresponding allylic zinc chloride produces only a trace of this coupled product. These zinc allylic reagents which can also add readily to aldehydes and methyl ketones with high diastereoselectivity (Scheme 74) and react regioselectively with acid chlorides to afford $\beta, \gamma$-unsaturated ketones, are more fully formulated as 
$\left(\mathrm{R}^{*}\right)$ ZnOPiv $\cdot \mathrm{Mg}(\mathrm{OPiv}) \mathrm{X} \cdot \mathrm{nLiCl}\left(\right.$ where $\mathrm{R}^{*}=$ various allylic groups; $\mathrm{X}=\mathrm{Cl}, \mathrm{Br}$, or I). Note that although these solid zinc allylic reagents react rapidly with air and moisture, they are stable for an extended period of time under an argon blanket.

Scheme 74. Preparation and representative reactions of a solid zinc allylic reagent.
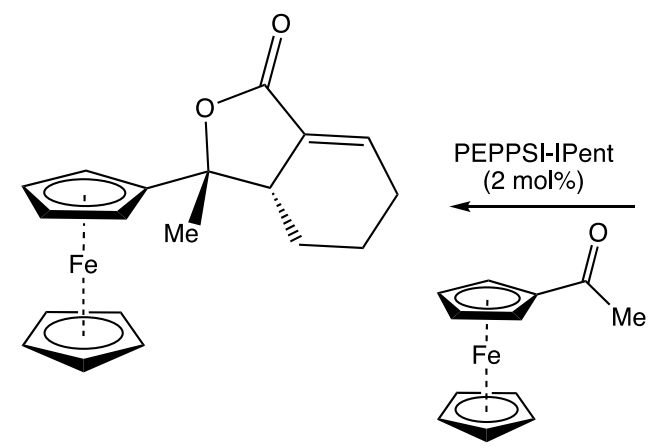<smiles>CCOC(=O)C1=CCCCC1</smiles>

$$
\downarrow \begin{gathered}
\mathrm{Zn} \\
\mathrm{LiCl} \\
\mathrm{Mg}(\mathrm{OPiv})_{2}
\end{gathered}
$$

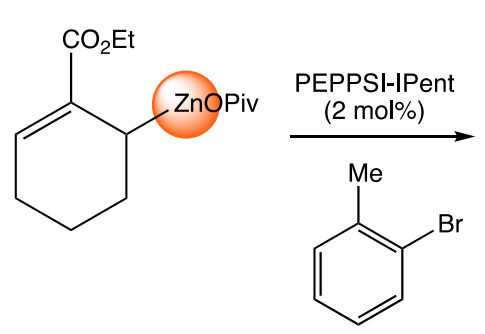<smiles>CCOC(=O)C1=CCCCC1c1ccccc1C</smiles>

Related allylic-free zinc pivalate reagents of general formula "RZnOPiv·Mg(OPiv)X·nLiCl" (where R = aryl, heteroaryl, or benzyl; $\mathrm{X}=\mathrm{Cl}, \mathrm{Br}$, or I) have been synthesized by a one-pot procedure mixing organic halides $(\mathrm{RX} ; \mathrm{X}=\mathrm{Cl}, \mathrm{Br})$ with magnesium turnings (generating Grignard reagents $\mathrm{RMgX}$ ) and the THF-soluble salt $\mathrm{Zn}(\mathrm{OPiv})_{2} \cdot 2 \mathrm{LiCl}$ in a 1.0:2.5:1.5 stoichiometric ratio in THF solution. These reagents have been successfully employed in a wide variety of Negishi cross-coupling and carbonyl addition reactions. ${ }^{129-131}$ Salt-supported aryl- and heteroarylzinc pivalates have also been aminated with $O$-benzoylhydroxylamines at ambient temperature in 2-4 hours. A novel feature is the catalysts employed, namely the mixed salt $\mathrm{CoCl}_{2} \cdot 2 \mathrm{LiCl}$ (loading 2.5-5.0\%) that produces the best yields of the tertiary arylated or 
heteroarylated amine products. Diarylamines and aryl(heteroaryl)amines can also be produced using this electrophilic amination procedure. ${ }^{132}$

NMR (including DOSY) spectroscopic and ESI mass spectroscopic investigations have shed more light on these synergistic "RZnOPiv·Mg(OPiv)X·nLiCl" mixtures. Using the para-tolyl Grignard reagent $\mathrm{Me}\left(p-\mathrm{C}_{6} \mathrm{H}_{4}\right) \mathrm{MgCl}$ as a case study it was found that its reaction with zinc pivalate underwent full transmetalation to the corresponding paratolyl zinc chloride reagent and magnesium pivalate, rather than to the products of partial transmetalation, namely para-tolyl zinc pivalate and the magnesium chloro pivalate (Scheme 75). ${ }^{133}$

Scheme 75. In the metathesis reaction between $\mathrm{Me}\left(p-\mathrm{C}_{6} \mathrm{H}_{4}\right) \mathrm{MgCl}$ and zinc pivalate the two pivalate ligands are transferred to the magnesium center.
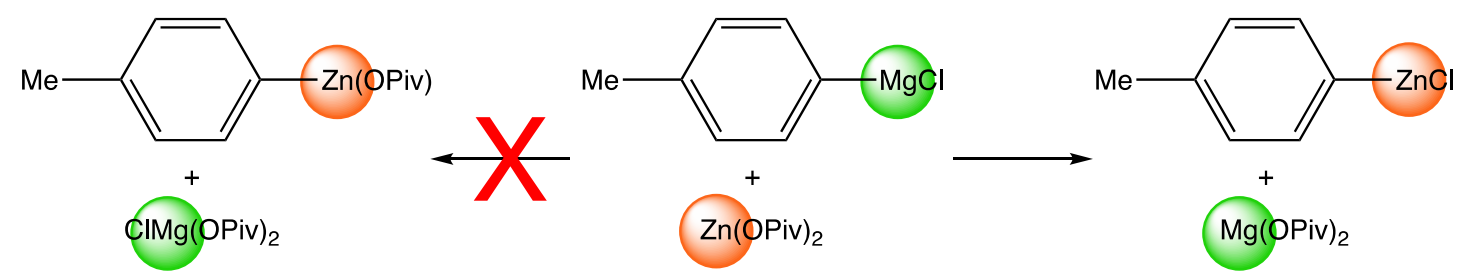

A novel feature of the aryl, heteroaryl and benzyl zinc pivalates is that they can resist attack by moist air over an extended period in contrast to more conventional organometallic compounds that decompose rapidly in contact with moist air. This can be deduced from the comparative data in Table 1, where Negishi cross-coupling reactions between zinc pivalate reagents and 4-bromoanisole show comparable yields under argon or in the presence of air.

Table 1. Reactivity of turbo-zinc reagents towards cross-coupling with 4-bromoanisole 


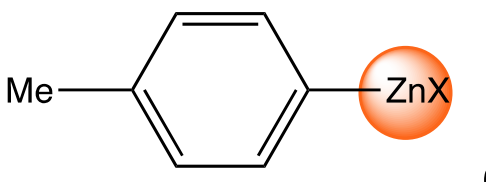

$3 \% \mathrm{Pd}(\mathrm{OAc})_{2}$ $6 \%$ DavePhos $1.5 \mathrm{~h}, 50^{\circ} \mathrm{C}$
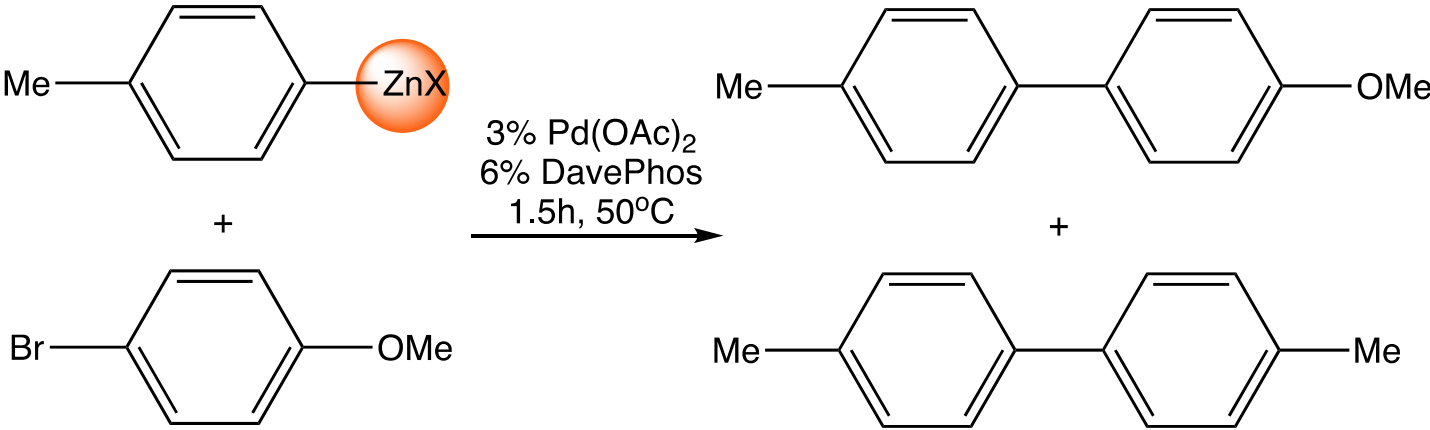

\section{Entry} $\mathbf{x}$

Solvent Conditions Yield of cross-

coupled product

\begin{tabular}{|c|c|c|c|c|}
\hline 1 & $\mathrm{Cl} \cdot \mathrm{MgCl}_{2} \cdot \mathrm{LiCl}$ & THF & Argon & 85 \\
\hline 2 & $\mathrm{Cl} \cdot \mathrm{MgCl}_{2} \cdot \mathrm{LiCl}$ & THF & air & 77 \\
\hline 3 & $\mathrm{Cl} \cdot \mathrm{MgCl}_{2} \cdot \mathrm{LiCl}$ & EtOAc & Argon & 91 \\
\hline 4 & $\mathrm{Cl} \cdot \mathrm{MgCl}_{2} \cdot \mathrm{LiCl}$ & EtOAc & air & 78 \\
\hline 5 & $\mathrm{Cl} \cdot \mathrm{Mg}(\mathrm{OPiv})_{2} \cdot \mathrm{LiCl}$ & THF & Argon & 82 \\
\hline 6 & $\mathrm{Cl} \cdot \mathrm{Mg}(\mathrm{OPiv})_{2} \cdot \mathrm{LiCl}$ & THF & air & 88 \\
\hline 7 & $\mathrm{Cl} \cdot \mathrm{Mg}(\mathrm{OPiv})_{2} \cdot \mathrm{LiCl}$ & EtOAc & Argon & 85 \\
\hline 8 & $\mathrm{Cl} \cdot \mathrm{Mg}(\mathrm{OPiv})_{2} \cdot \mathrm{LiCl}$ & EtOAc & air & 81 \\
\hline 9 & $\mathrm{Cl} \cdot \mathrm{LiCl}$ & THF & Argon & 86 \\
\hline 10 & $\mathrm{Cl} \cdot \mathrm{LiCl}$ & THF & air & 81 \\
\hline 11 & $\mathrm{Cl} \cdot \mathrm{LiCl}$ & EtOAc & Argon & trace \\
\hline 12 & $\mathrm{Cl} \cdot \mathrm{LiCl}$ & EtOAc & air & trace \\
\hline 13 & OPiv.LiOPiv & THF & Argon & 82 \\
\hline 14 & OPiv.LiOPiv & THF & air & 88 \\
\hline 15 & OPiv.LiOPiv & EtOAc & Argon & trace \\
\hline 16 & OPiv-LiOPiv & EtOAc & air & trace \\
\hline
\end{tabular}

Experiments gauging air sensitivity revealed that whereas (aryl) $\mathrm{ZnCl}$ reagents show inherently better stability towards air ${ }^{134}$ than (alkyl)ZnCl reagents; remarkably, the presence of separated $\mathrm{Mg}(\mathrm{OPiv})_{2}$ can supplement this stability by mopping up $\mathrm{OH}^{-}$or $\mathrm{O}_{2}{ }^{-}$contaminants as well as capturing and holding on to $\mathrm{H}_{2} \mathrm{O}$ molecules, making them 
less accessible for hydrolyzing C-Zn bonds. ${ }^{133}$ On the contrary lithium chloride seems to lessen the stability of organozinc pivalate reagents towards moisture because $\mathrm{LiCl}$ can be incorporated within the $\mathrm{Zn}$ structure and thus brings $\mathrm{H}_{2} \mathrm{O}$ molecules into close proximity to $\mathrm{Zn}-\mathrm{C}$ bonds. This study also explained the synergistic solubilizing effect of lithium chloride on $\mathrm{Zn}(\mathrm{OPiv})_{2}$, which in the absence of the lithium salt is essentially insoluble in THF. The origin of this effect lies in the formation of the molecular complex $\left[(\mathrm{THF})_{2} \mathrm{Li}_{2}(\mu-\mathrm{Cl})_{2}(\mu-\mathrm{OPiv})_{2} \mathrm{Zn}\right](\mathbf{1 1 2})$, the structure of which was determined from an Xray crystallographic study (Figure 26).

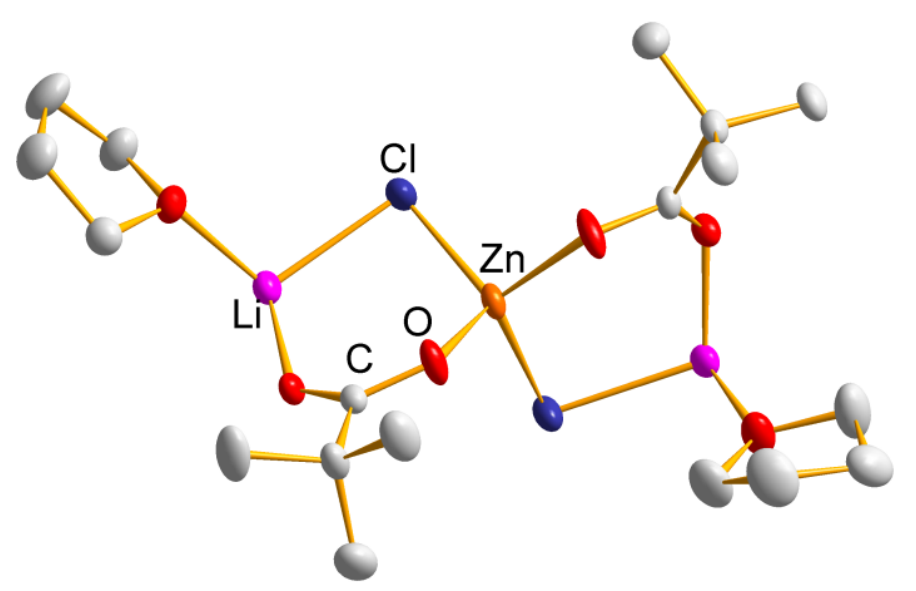

Figure 26. Molecular structure of $\left[(\mathrm{THF})_{2} \mathrm{Li}_{2}(\mu-\mathrm{Cl})_{2}(\mu \text {-OPiv })_{2} \mathrm{Zn}\right](\mathbf{1 1 2})$, explaining the solubility of zinc pivalate in the presence of lithium chloride.

This pivalate-induced enhanced air and moisture resistance of organozinc reagents extends to solid polyfunctional alkynylzinc pivalates, which can be made in high yield from functionalized alkynes of general formula $\mathrm{FG}-\mathrm{C} \equiv \mathrm{CH}$ ( $\mathrm{FG}=$ functional group) by deprotonation using the turbo zinc-magnesium base $\mathrm{TMPZnCl} \cdot \mathrm{Mg}(\mathrm{OPiv})_{2}(\mathbf{1 1 3}) .{ }^{135}$ Note that using the alternative composition TMPZnOPiv.LiCl (114) to prepare the alkynylzinc pivalate containing lithium chloride showed less air stability (44 \% activity after $4 \mathrm{~h}$ in air) compared to those made using LiCl-free TMPZnCl·Mg(OPiv $)_{2}(\mathbf{1 1 5}$, up 
to $90 \%$ activity under the same conditions, Scheme 76). These polyfunctional alkynylzinc pivalates show impressive reactivity in various $\mathrm{C}-\mathrm{C}$ bond-forming reactions including Pd-catalyzed Negishi cross-coupling with an assortment of aryl halides, acylation, allylation, and aldehyde addition as well as 1,3-dipolar cycloadditions.

Scheme 76. Preparation of functionalized alkynylzinc pivalate reagents using 115.
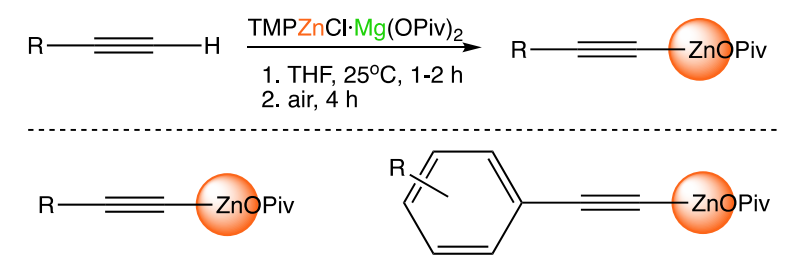

\begin{tabular}{|c|c|}
\hline $\mathbf{R}$ & yield (\%) \\
\hline $\mathrm{SiMe}_{3}$ & 90 \\
\hline${ }^{n} \mathrm{Bu}$ & 71 \\
\hline $\mathrm{CHex}$ & 73 \\
\hline $\mathrm{CO}_{2} \mathrm{Et}$ & 82 \\
\hline
\end{tabular}

\begin{tabular}{|c|c|}
\hline $\mathbf{R}$ & yield (\%) \\
\hline $4-\mathrm{OMe}$ & 80 \\
\hline $2-\mathrm{CO}_{2} \mathrm{Et}$ & 90 \\
\hline $4-\mathrm{CO}_{2} \mathrm{Et}$ & 90 \\
\hline $4-\mathrm{CN}$ & 87 \\
\hline $3-\mathrm{CO}^{\prime} \mathrm{Pr}$ & 86 \\
\hline $4-{ }^{\mathrm{t}} \mathrm{Bu}$ & 82 \\
\hline
\end{tabular}

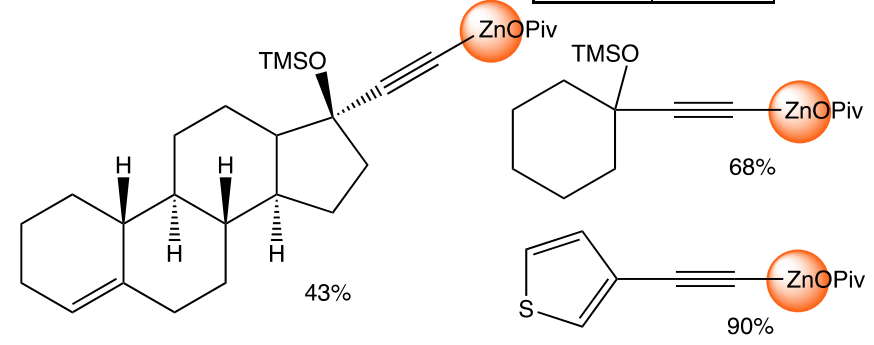

The class of solid turbo-zinc reagents has also been extended to enolates. These have been prepared by reaction of a series of $\mathrm{N}$-morpholino amides with TMPZnCl-LiCl and $\mathrm{Mg}(\mathrm{OPiv})_{2}$ in THF solution at $25^{\circ} \mathrm{C}$ (Scheme 77$) . .^{136}$

Scheme 77. Preparation of enolate-based zinc pivalate reagents.

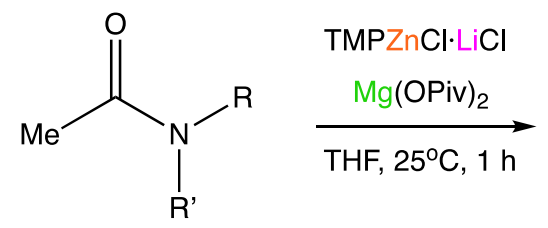<smiles>O=C(CC1CCCCC1)N(P)P</smiles> 
Solid zinc pivalate ketone or ester enolates show unsatisfactory stability towards air and moisture and were deemed unsuitable for use in synthetic campaigns so preference was given to these $\mathrm{N}$-morpholino amides. As with the aforementioned alkynyl, aryl, benzyl and heteroaryl zinc pivalates, these zinc amide enolates also have the asset of enhanced air and moisture stability when in solid form $\left(t_{1 / 2}\right.$ in air lie in the range 1-3 h), making them in effect the first solid Reformatsky-type reagents with this advantageous property as such reagents are usually generated in situ. ${ }^{137}$ These enolates are effective in $\mathrm{Pd}$ - and Cu-catalyzed cross-couplings with (hetero)aryl bromides as well as allylic and benzylic halides (Scheme 78).

Scheme 78. Representative cross-coupling reactions between $\mathrm{N}$-morpholino amide zinc pivalates and various organic halides. 
<smiles>O=C(Cc1ccccc1)N1CCOCC1</smiles>

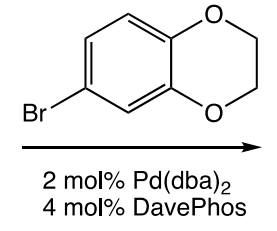<smiles>O=C(Cc1ccc2c(c1)OCCO2)N1CCOCC1</smiles><smiles>CC(C(=O)N1CCOCC1)C1CCCC1</smiles><smiles>CCCCCCCCCOc1ccc(I)cc1</smiles><smiles>CCCCOc1ccc(C(C)C(=O)N2CCOCC2)cc1</smiles><smiles>O=C(C(N=C(c1ccccc1)c1ccccc1)c1ccccc1)N1CCOCC1</smiles><smiles>N#Cc1ccccc1CCl</smiles><smiles>N#Cc1ccccc1CC(N=C(c1ccccc1)c1ccccc1)C(=O)N1CCOCC1</smiles><smiles>CC(C(=O)N1CCOCC1)c1ccccc1</smiles><smiles>CCOC(=O)C(=CC(=O)O)CBr</smiles><smiles>C=C(CC(C)C(=O)N1CCOCC1)C(=O)OC</smiles>

Note that for simplicity "RZnOPiv" is used to denote these enolates in the papers concerned, but that they also contain $\mathrm{Mg}(\mathrm{OPiv}) \mathrm{Cl}$ and $\mathrm{LiCl}$ salts too. The utility of the zinc amide enolates was demonstrated by preparing the anti-breast-cancer drug candidate 5-methoxy-2-(4-methoxyphenyl)-1H-indole-3-carbaldehyde in six steps and $23 \%$ overall yield (Scheme 79$)$.

Scheme 79. Preparation of the anti-breast-cancer drug candidate 5-methoxy-2-(4methoxyphenyl)-1H-indole-3-carbaldehyde.<smiles>COc1ccc(N=[N+]([O-])c2ccc(CC(=O)N3CCOCC3)cc2)cc1</smiles><smiles>C#C[C@H](C)c1ccc(OC)cc1/C(=C\C(=O)N1CCOCC1)c1ccc(OC)cc1</smiles> 


\subsubsection{Turbo Reagents in Metal-Halogen Exchange Reactions}

Complementary to lithium-hydrogen exchange reactions, lithium-halogen exchange is also one of the cornerstone reactions in organic synthesis, ${ }^{138}$ first discovered independently in the 1930 s by two goliaths of organolithium chemistry, namely Gilman ${ }^{139}$ and Wittig. ${ }^{140}$ Despite its vast utilization, in general this reaction has proved less successful for synthesizing polyfunctional lithium intermediates at reasonable temperatures due to the high reactivity and concomitantly high polarity of the Li-C bond. Substituting lithium by magnesium lowers the polarity and thus lowers the reactivity; hence magnesium-iodine exchange reactions are more suitable for the synthesis of polyfunctional organomagnesium intermediates. ${ }^{141,142}$ But with lower reactivity comes slower kinetics, so while lithium-halogen exchange is generally a rapid process, magnesium-halogen (iodine or bromine) exchange reactions are comparatively slow. This is where lithium chloride mediation plays a beneficial role exemplified by ${ }^{i} \mathrm{PrMgCl} \cdot \mathrm{LiCl}(\mathbf{1 0 8})$, a Grignard reagent - salt co-complex or better still, because of its reaction accelerating effect, a turbo Grignard reagent (zoom zoom). This acceleration is prominent in magnesium-bromine and magnesium-iodine exchange processes. ${ }^{143-147}$ An early example illustrates some of the advantages of using $108 .{ }^{148}$ Ultrasensitive when metalated by a conventional metalating agent and therefore prone to rapid decomposition, the triazine substrate poses other challenges, as it possesses both an iodine and bromine substituent (Scheme 80). However, the turbo Grignard reagent selectively performs magnesium-iodine exchange, albeit at $-40^{\circ} \mathrm{C}$ in THF to form an intermediate Grignard reagent with a polyfunctionalized aryl group which, on heating at $50{ }^{\circ} \mathrm{C}$, undergoes a cyclization to give the carbazole product in high yield. 
Scheme 80. An example of metal-halogen exchange using a turbo-Grignard reagent in the preparation of carbazole.<smiles>Cc1cccc(-c2c(Cl)cccc2-c2cccc(Br)c2/N=N/N2CCCC2)c1N=NN1CCCC1</smiles>

Other sensitive substrates such as heterocyclic isoxazoles can also be magnesiated using ${ }^{\mathrm{P} r M g C l} \cdot \mathrm{LiCl}$ without ring opening (Scheme 81), ${ }^{149}$ making them accessible for onward functionalization via reaction with electrophiles. Such functionalizations of isoxazoles are important given the prominence of the five-membered $\mathrm{NOC}_{3}$ ring scaffold in commercial drugs. ${ }^{150}$

Scheme 81. Representative turbo halogen-magnesium exchange reaction of an isoxazole.<smiles>Cc1cnoc1</smiles>

Crystallographic evidence showing the structural complexity of the turbo Grignard reagent $\mathbf{1 0 8}$ has come from an X-ray crystallographic study of crystals grown from a THF solution layered with ether at ambient temperature. ${ }^{151}$ Surprisingly these crystals did not contain lithium chloride, but instead contained magnesium chloride as the additive in $\left[{ }^{i} \mathrm{PrMgCl}(\mathrm{THF})\right]_{2}\left[\mathrm{MgCl}_{2}(\mathrm{THF})_{2}\right]_{2}$ (116). This can be regarded as a dimeric cocomplex between the Grignard reagent [ $\mathrm{PrMgCl}(\mathrm{THF})]$ and a molecular THF- 
solvated form of magnesium chloride. Crystalline $\mathbf{1 1 6}$ exhibits an open cube motif, whereby two cubes share a $\mathrm{MgClMgCl}$ face, with "missing" metal atoms at the opposing end corners of the cubes (Figure 27).

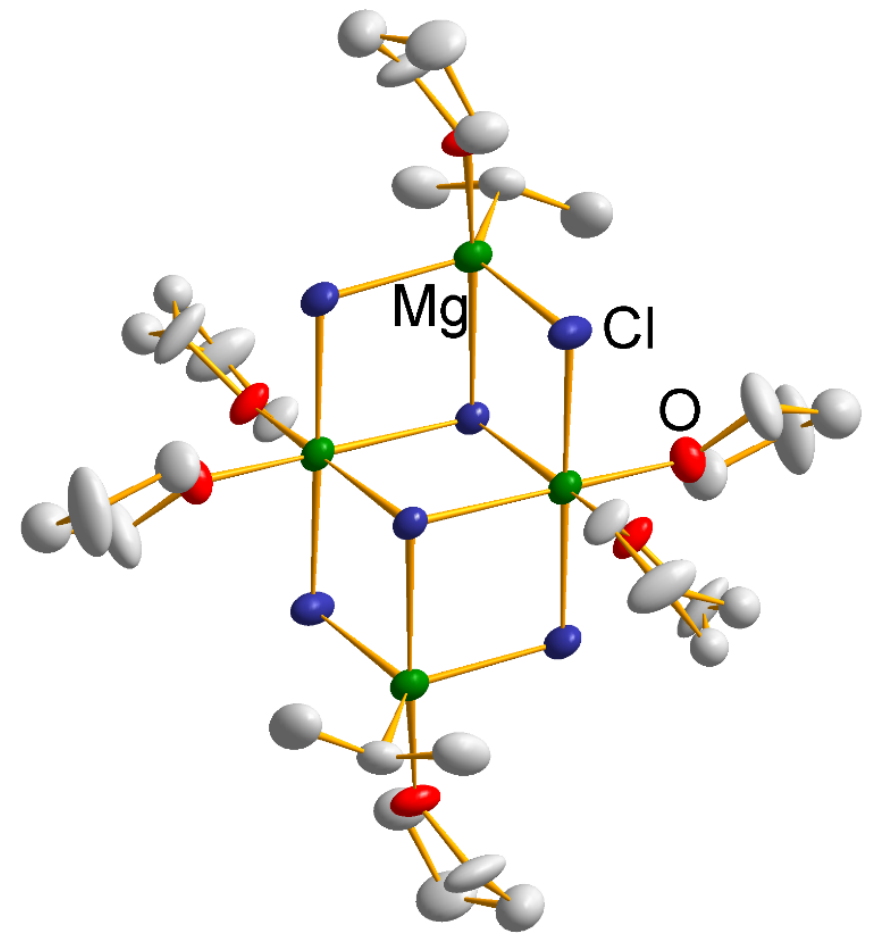

Figure 27. Molecular structure of $\left[{ }^{i} \mathrm{PrMgCl}(\mathrm{THF})\right]_{2}\left[\mathrm{MgCl}_{2}(\mathrm{THF})_{2}\right]_{2}$ (116), a surprising product obtained from a turbo-Grignard reagent THF solution.

The outermost $\mathrm{Mg}$ atoms bear the terminal iso-propyl groups with other coordination sites filled by THF molecules. This is a known crystallographic structural type with examples found in conventional Grignard reagents $\mathrm{RMgCl}$ in $\mathrm{THF}\left(\mathrm{R}=\mathrm{Me},{ }^{t} \mathrm{Bu}, \mathrm{Ph}\right.$, $\left.\mathrm{PhCH}_{2}\right) .{ }^{152} \mathrm{~A}$ driving force for this type of cocomplex could be the stronger Lewis acidity of the $\mathrm{Mg}$ center in $\mathrm{MgCl}_{2}$ in comparison to the $\mathrm{Li}$ center in $\mathrm{LiCl}$. An extension of the Schlenk equilibrium has been hypothesized to explain the existence of this cocomplex, though note it is not balanced (Scheme 82). The distribution of products seems even broader given that when dioxane was introduced to a THF solution of 
${ }^{i} \mathrm{PrMgCl} \cdot \mathrm{LiCl}$, the crystalline complexes $\left[\mathrm{LiCl}(\mathrm{THF})_{2}\right]_{2}$ and bis-alkyl $\left[{ }^{i} \mathrm{Pr}_{2} \mathrm{Mg} \text { (dioxane) }\right]_{\infty}$ were isolated from it.

Scheme 82. Proposed Schlenk-type equilibria taking place in turbo-Grignard reagent solutions in THF that deposit $\left[{ }^{i} \mathrm{PrMgCl}(\mathrm{THF})\right]_{2}\left[\mathrm{MgCl}_{2}(\mathrm{THF})_{2}\right]_{2}(\mathbf{1 1 6})$.

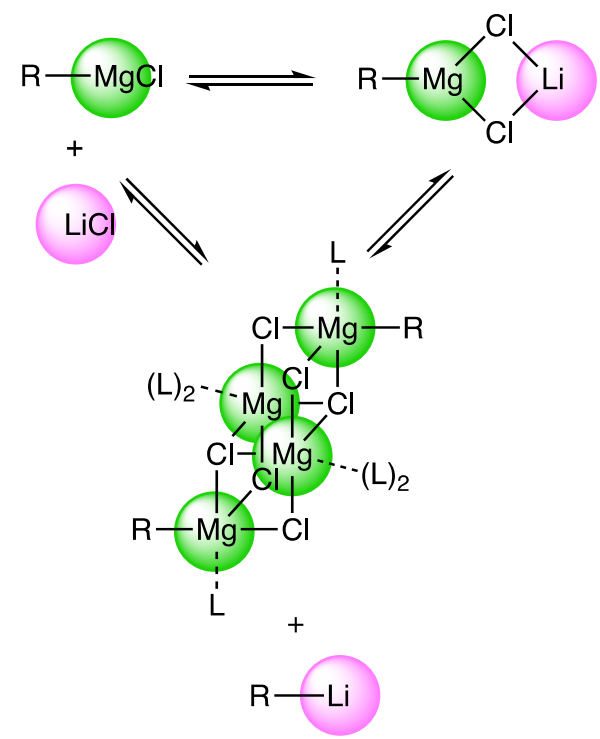

Turbo Grignard reagent reactions can also be scaled up making them attractive to industry. For example, Merck research laboratories have demonstrated the use of $\mathbf{1 0 8}$ on $100 \mathrm{~g}$ scale in the preparation of $\alpha$-hydroxyacetophenones, compounds that provide entry into a broad range of chiral molecules. The target $\alpha$ hydroxyacetophenones are made following magnesium-bromide exchange with 1bromo-3,5-difluorobenzene which is complete in one hour at $25^{\circ} \mathrm{C}$, transmetalation with $\mathrm{ZnCl}_{2}$ then $\mathrm{Cu}(\mathrm{I})$-catalyzed reaction with acetoxyacetyl chloride and acid hydrolysis (Scheme 83). The synergistic effect of the $\mathrm{LiCl}$ mediation was clear to the authors who stated that "the use of ${ }^{i} \mathrm{PrMgCl} \cdot \mathrm{LiCl}$ rather than the conventional Grignard reagent ${ }^{i} \mathrm{PrMgCl}$ led to more predictable and better results for $\mathrm{Mg}-\mathrm{X}$ exchange for the substrates studied" ${ }^{153}$ 
Scheme 83. Preparation of an $\alpha$-hydroxyacetophenone with the initial step a magnesium-halogen exchange involving a turbo-Grignard reagent, 108.

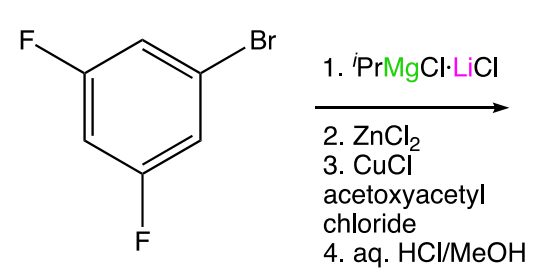<smiles>O=C(CO)c1cc(F)cc(F)c1</smiles>

Taking advantage of the classic Schlenk equilibrium, ${ }^{i} \mathrm{PrMgCl} \cdot \mathrm{LiCl}$ can be converted to the dialkyl derivative ${ }^{i} \mathrm{Pr}_{2} \mathrm{Mg} \cdot 2 \mathrm{LiCl}$ (117, Scheme 84). The latter reagent reacts faster and in a shorter timescale than the former turbo Grignard reagent as observed in reactions with 4-bromoanisole ( $31 \%$ conversion after $24 \mathrm{~h}$ at $25{ }^{\circ} \mathrm{C}$ versus $100 \%$ conversion after $10 \mathrm{~h}$ at $\left.25^{\circ} \mathrm{C}\right) .{ }^{144}$

Scheme 84. Schlenk equilibrium for the turbo-Grignard reagent ${ }^{i} \mathrm{PrMgCl} \cdot \mathrm{LiCl}(108)$.

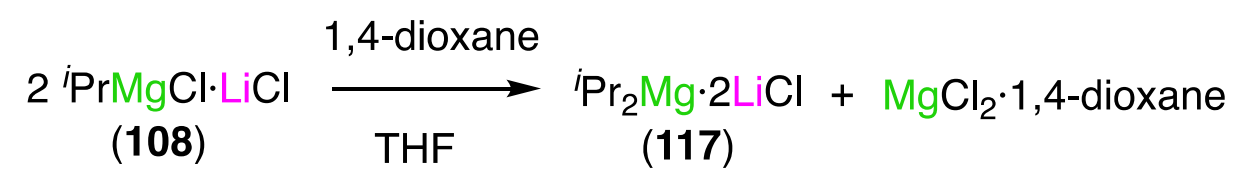

Looking beyond lithium chloride for other magnesium-halogen exchange accelerators, beneficial synergistic outcomes have also been established combining ${ }^{i} \mathrm{Pr}_{2} \mathrm{Mg}$ with enantiomerically pure lithium alkoxides leading to the desymmetrization of prochiral bis(bromoaryl)alcohols. Using (ortho-bromophenyl)ethanol as a model alcohol, a 96 \% complete magnesium-bromine exchange using the additive $\mathrm{LiOCH}_{2} \mathrm{CH}_{2} \mathrm{NMe}_{2}$ takes place in ether solution within $6 \mathrm{~h}$ at $25{ }^{\circ} \mathrm{C}$; whereas the analogous ${ }^{i} \mathrm{PrMgCl} \cdot \mathrm{LiO}\left(\mathrm{CH}_{2}\right)_{2} \mathrm{NMe}_{2}$ mixture proved totally inert. Bifunctional bromoaryl alcohols (e.g., binol-type) were used to enable two-point binding such that the chiral moiety 
would bind sufficiently strongly to the magnesium during the intramolecular exchange process. This method worked efficiently during the enantioselective synthesis of anticholinergic and antihistaminic pharmaceutical (R)-orphenadrine (Scheme 85). ${ }^{154}$

Scheme 85. Enantioselective synthesis of $(R)$-orphenadrine using a bimetallic mixture of a dialkylmagnesium complex and a lithium binol-type compound.<smiles>COC1=CC=CC2=C(c3c(OC)ccc4ccccc34)c3ccccc3C12c1ccccc1</smiles><smiles>CN(C)CCO[C@H](c1ccccc1)c1ccccc1Br</smiles>

Earlier work determined synergistic effects in magnesium-halogen exchange reactions using diorganomagnesium complexes paired with different alkali metal alkoxides $\left(\mathrm{MOR}=\mathrm{LiO}^{\mathrm{B}} \mathrm{Bu}, \mathrm{KOPh}, \mathrm{KOMe}\right)$. Thought to involve magnesiate complexes of formula $\left[\mathrm{M}^{+}\left(\mathrm{R}_{2} \mathrm{MgOR}\right)^{-}\right]$, it was suggested that in side reactions producing benzynes the species abstracting a hydrogen was not the alkyl group $\mathrm{R}$ but the alkoxide group OR, which was rendered more reactive by incorporation into an organomagnesiate structure. ${ }^{155}$

With a key factor behind the success of turbo Grignard reagents being the enhanced solubility provided by the lithium chloride additive, it was logical to prepare modifications of this class of compound with other functional groups with excellent solubility characteristics. This has been demonstrated emphatically with the employment of an additive derived from a long chain aliphatic alcohol ROH in both the turbo Grignard-type reagent ${ }^{s} \mathrm{BuMgOR} \cdot \mathrm{LiOR}(\mathbf{1 1 8})$ and its dialkylmagnesium form 
${ }^{s} \mathrm{Bu}_{2} \mathrm{Mg} \cdot 2 \mathrm{LiOR}\left(\mathbf{1 1 9}\right.$, where $\mathrm{R}=$ 2-ethylhexyl). ${ }^{156}$ Prepared via commercially available chemicals (Scheme 86), these heterometallic, heteroleptic reagents provide a rich source of aryl- and heteroaryl-magnesium reagents when treated with aryl- and heteroaryl-halides, which in turn can be trapped by a variety of electrophiles.

Scheme 86. Preparation of the turbo-Grignard reagent ${ }^{s}$ BuMgOR-LiOR (118) and its dialkylmagnesium modification ${ }^{5} \mathrm{Bu}_{2} \mathrm{Mg} \cdot 2 \mathrm{LiOR}$ (119).

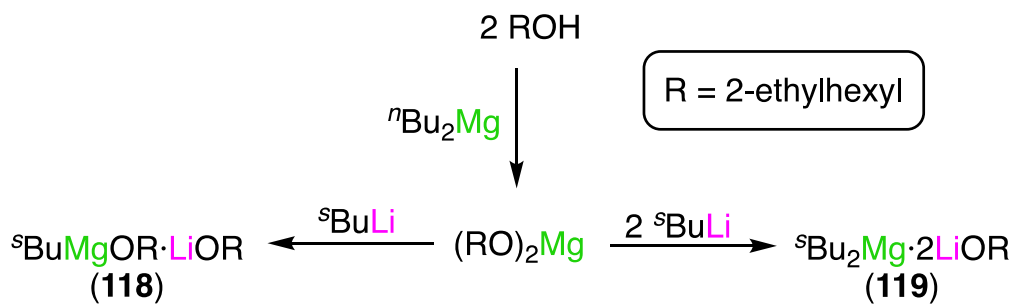

The novelty of these complex alkoxides lies in their ability to carry out reactions in toluene solution (most such reactions are performed in THF) (Scheme 87a), the greater speed of their magnesium-bromine exchanges compared to that with related chloride complexes ${ }^{i} \mathrm{PrMgCl} \cdot \mathrm{LiCl}(\mathbf{1 0 8})$ or ${ }^{\mathrm{S}} \mathrm{Bu} \mathrm{u}_{2} \mathrm{Mg} \cdot 2 \mathrm{LiCl}(\mathbf{1 2 0}$, Scheme 87b), and their capacity to effect more challenging magnesium-chloride exchanges with a series of electron-rich aryl chlorides (Scheme 87c).

Scheme 87. (a) Typical reactivity of the turbo-Grignard reagent ${ }^{s}$ BuMgOR-LiOR (119) in toluene solution; (b) its greater efficiency in reactions with 4-bromoanisole; and (c) example of its capacity to exchange with chloro substrates. 


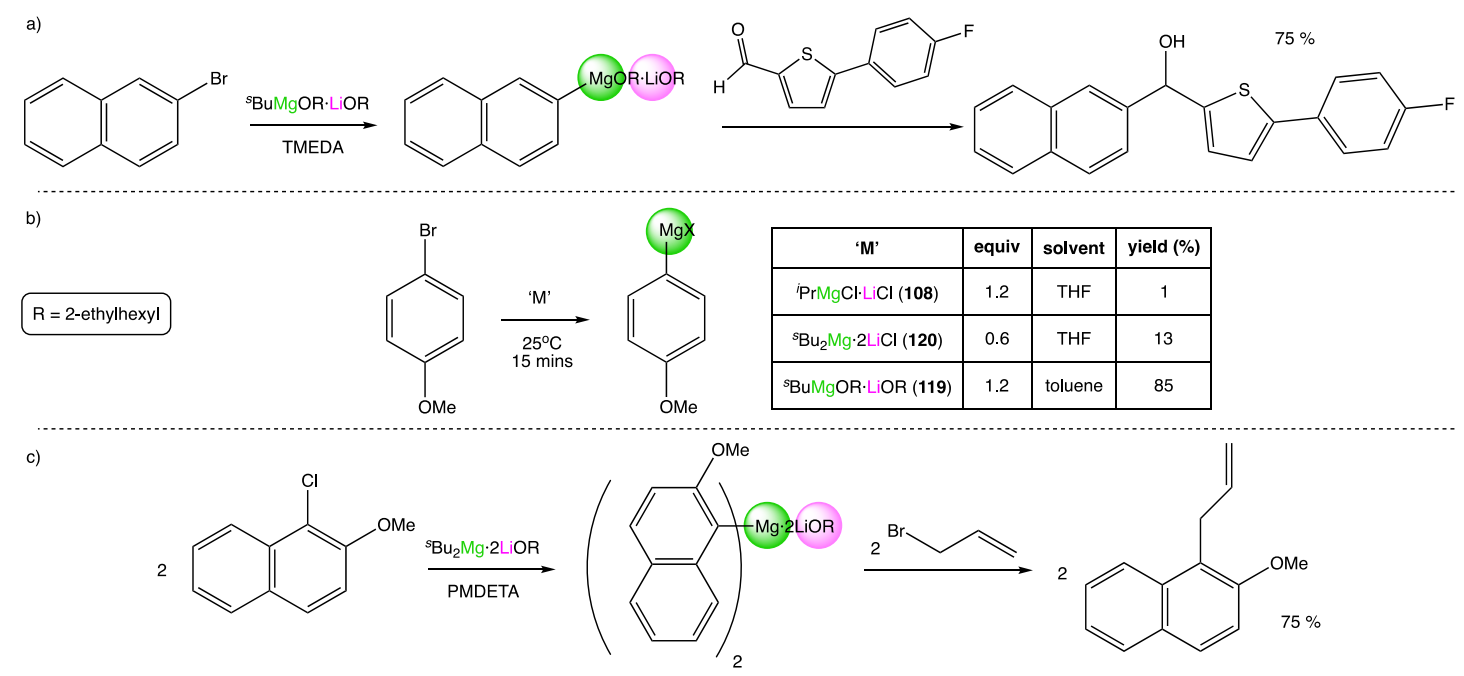

\subsubsection{Reactions Performed Under Continuous Flow Conditions}

An emerging development in this area has been the use of continuous flow chemistry. The bimetallic salt $\mathrm{ZnCl}_{2} \cdot 2 \mathrm{LiCl}$ (as well as other salts such as $\mathrm{MgCl}_{2}, \mathrm{CuCN} \cdot 2 \mathrm{LiCl}$, and $\mathrm{LaCl}_{3} \cdot 2 \mathrm{LiCl}$ ) has been utilized as an in situ trap for LiTMP metalations of arenes and $\mathrm{N}$ heterocycles under convenient continuous flow conditions. ${ }^{157}$ The mixed-metal formulation $\mathrm{ZnCl}_{2} \cdot 2 \mathrm{LiCl}$ is used since it exhibits good solubility in THF. These are kinetic metalations because LiTMP first deprotonates the unsaturated substrate before the transmetalation with the salt takes place (compare with trans-metal-trapping where complete transmetalation is not reached but stalls at a crossover stage, see section 2.5.3). Performing these reactions in continuous flow apparatus can increase significantly the synthetic scope of such metalations, the yields obtained and the scale-up opportunities of the in situ trapping reactions. For example, combining $\mathrm{ZnCl}_{2} \cdot 2 \mathrm{LiCl}$ and ethyl 4-bromobenzoate in a 1:2 stoichiometric ratio in THF with LiTMP (1.5 equivalents) in flow apparatus for $40 \mathrm{~s}$ at $0{ }^{\circ} \mathrm{C}$ produces after normal bench iodolysis the aryl iodide in 95\% yield; whereas carried out in a conventional Schlenk tube at $-78{ }^{\circ} \mathrm{C}$ the best yield of the iodide product was only $53 \%$. (Scheme 88 ). 
Scheme 88. Comparison of conventional batch and flow procedures for metalation/iodolysis of ethyl 4-bromobenzoate.<smiles>CCOC(=O)c1ccc(Br)cc1</smiles>

1. $0.5 \mathrm{ZnCl}_{2} \cdot 2 \mathrm{LiCl}$

2. 1.5 TMPLi

3. $\mathrm{I}_{2}$<smiles>CCOC(=O)c1ccc(Br)cc1I</smiles>

\begin{tabular}{|c|c|c|c|}
\hline conditions & temp $\left({ }^{\circ} \mathrm{C}\right)$ & time & yield $(\%)$ \\
\hline batch & -78 & 5 mins & 53 \\
\hline flow & 0 & $40 \mathrm{~s}$ & 95 \\
\hline
\end{tabular}

Proof that the active base in this reaction is LiTMP came from the fact that the turbozinc reagent $\mathrm{TMP}_{2} \mathrm{Zn} \cdot 2 \mathrm{LiCl}(\mathbf{1 0 6})$, which would be the product in the absence of ethyl 4-bromobenzoate does not deprotonate the aryl bromide under the same reaction conditions. These kinetically controlled in situ metalations can also give rise to special regioselectivities. With the three-component turbo-organomanganese base $\mathrm{TMP}_{2} \mathrm{Mn} \cdot 2 \mathrm{MgCl}_{2} \cdot 4 \mathrm{LiCl}$ (121), 3-substituted ethyl benzoates are generally metalated at the 2-position, but under these kinetic flow conditions LiTMP can metalate at the 6position with onward Negishi cross-coupling or copper-mediated acylation leading to trisubstituted arenes (Scheme 89).

Scheme 89. Sequential metalation and acylation of ethyl 3-fluorobenzoate.

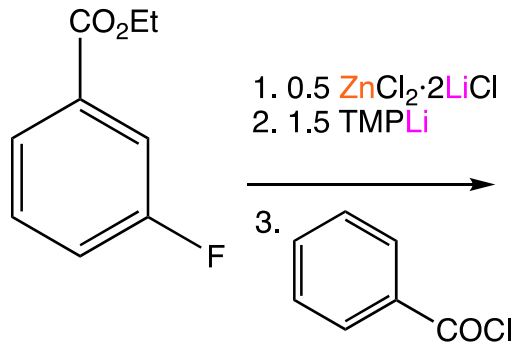<smiles>CCOC(=O)c1cc(F)ccc1C(=O)c1ccccc1</smiles>

Less expensive than the more sterically hindered base LiTMP, lithium dicyclohexylamide $\left[\left(\mathrm{Cy}_{2} \mathrm{NLi}\right)_{n}\right]$ allows fast and convenient $\left(40 \mathrm{~s}, 0^{\circ} \mathrm{C}\right)$ salt-based in situ trapping flow metalations of assorted functionalized arenes, heteroarenes and 
acrylate substrates. ${ }^{158}$ The in situ trapping can be accomplished by different metal salts (i.e., $\mathrm{ZnCl}_{2} \cdot 2 \mathrm{LiCl}, \mathrm{MgCl}_{2}$, or $\mathrm{LaCl}_{3} \cdot 2 \mathrm{LiCl}$ ). The intermediate organozinc, organomagnesium or organolanthanum complexes are subsequently trapped with various electrophiles in high yields. Even hypersensitive substrates that generally prove awkward to metalate such as 2-fluoropyridine, undergo a smooth zincation or magnesiation (here in position 3 ) in the company of the metal salt $\mathrm{ZnCl}_{2} \cdot 2 \mathrm{LiCl}$ and intercepting with ethyl 4-iodobenzoate affords the disubstituted pyridine (Scheme 90). Other continuous flow magnesiations using TMPMgCl-LiCl (104) or zincations using TMPZnCl-LiCl (106) have been reported for acrylonitriles, acrylates, and nitroolefins. $^{159}$

Scheme 90. Flow metalation and Pd cross-coupling of 2-fluoropyridine.<smiles>Fc1ccccn1</smiles><smiles>CCOC(=O)c1ccc(I)cc1</smiles><smiles>CCOC(=O)c1ccc(-c2cccnc2F)cc1</smiles>

This flow methodology has been extended to the bis-amidozinc, bis-lithium chloride complex $\left(\mathrm{Cy}_{2} \mathrm{~N}\right)_{2} \mathrm{Zn} \cdot 2 \mathrm{LiCl}(\mathbf{1 2 2})$, which is made by a metathesis reaction in THF solution from the corresponding lithium amide and dispensed as a THF/DMPU (10:1) mixture [DMPU is 1,3-dimethyltetrahydropyrimidin-2(1H)-one] (Scheme 91). ${ }^{160}$

Scheme 91. Preparation of the turbo-zinc reagent $\left(\mathrm{Cy}_{2} \mathrm{~N}\right)_{2} \mathrm{Zn} \cdot 2 \mathrm{LiCl}(122)$. 


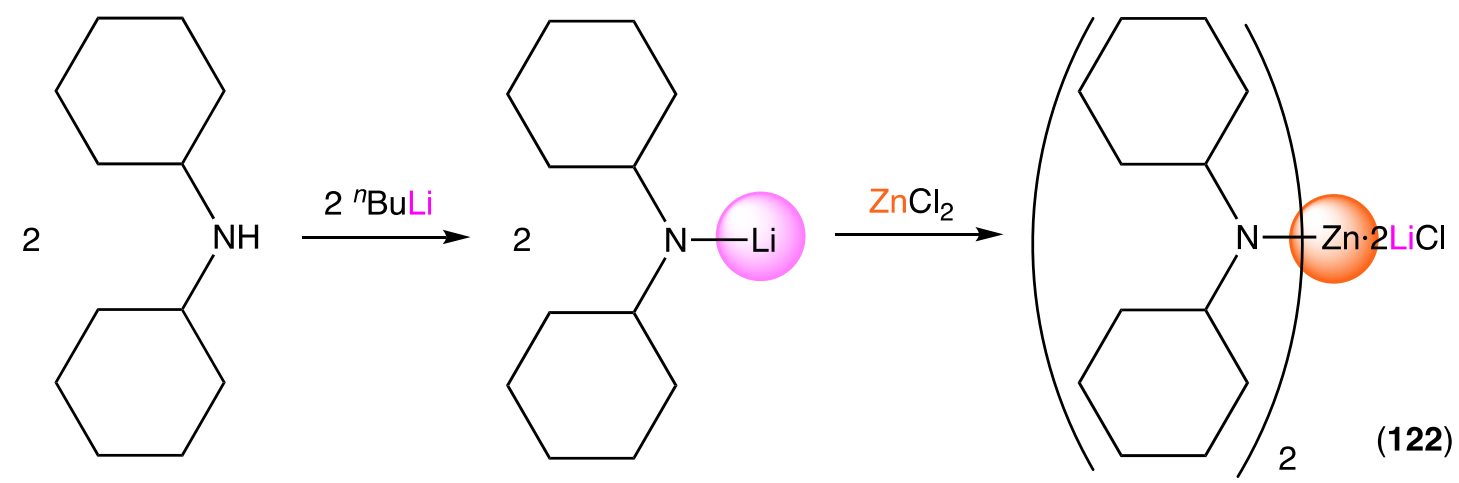

Applying flow conditions at temperatures in the range $25-100{ }^{\circ} \mathrm{C}$, sensitive arenes and hetero derivatives can be zincated rapidly (within 10 minutes) leading to synthetically useful polyfunctional zinc reagents. Note such metalations at the higher end of this temperature range are problematic under conventional batch conditions. Transferred to a batch reactor, the organozincated intermediates can be trapped by common electrophiles such as allylic bromides and aryl iodides in high yields. A key feature is that complementary thermodynamically controlled metalation regioselectivities are accessible for several substituted pyridines compared to commonly used TMP-magnesium and TMP-zinc turbo bases, which operate kinetically. For example, metalation of representative substituted pyridines with 104 or 106 take place at position 2 and position 6, respectively; whereas in contrast with these same pyridines the more economical base 122 used in a flow reactor $\left(60^{\circ} \mathrm{C}, 10\right.$ min) effects zincation at position 4 (Table 2).

Table 2. Selection of substituted pyridine metalation reactions by $\left(\mathrm{Cy}_{2} \mathrm{~N}\right)_{2} \mathrm{Zn} \cdot 2 \mathrm{LiCl}$ (122) under flow conditions $\left(60^{\circ} \mathrm{C}, 10 \mathrm{~min}\right)$ and onward electrophilic trapping reactions. 


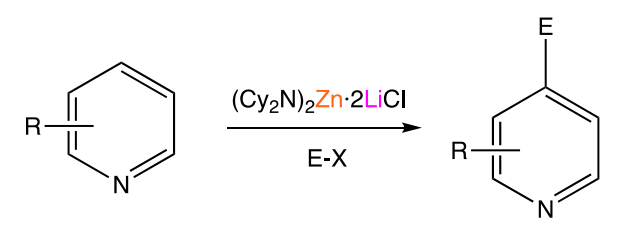

(\%)

Continuous flow protocols have also proved effective for the functionalization of unsymmetrical azobenzenes with focus on those with methoxy substituents. LiTMP lithiations occur under easy conditions $\left(0^{\circ} \mathrm{C}, 20 \mathrm{~s}\right)$, and different electrophiles (halide and carbonyl based) can be used. ${ }^{161}$ Synergistic in situ trapping approaches proved effective with sensitive substrates (containing CN or F substituents), whereby LiTMP 
is introduced to a mixture of the azobenzene and a salt $\left(\mathrm{ZnCl}_{2}\right.$ or $\left.\mathrm{MgCl}_{2} \cdot \mathrm{LiCl}\right)$ (Scheme 92). This continuous flow setup using an in situ trapping procedure with the $\mathrm{LiTMP} / \mathrm{ZnCl}_{2}$ combination has also been applied to the functionalization of $1,2-$ dicyanobenzene and related polyfunctionalized benzonitriles. ${ }^{162}$ Key advantages of this flow method are a broader reaction scope and employing fewer equivalents of the metalating agent and the zinc salt than the analogous conventional batch procedure.

Scheme 92. Flow functionalization reaction of an azobenzene.

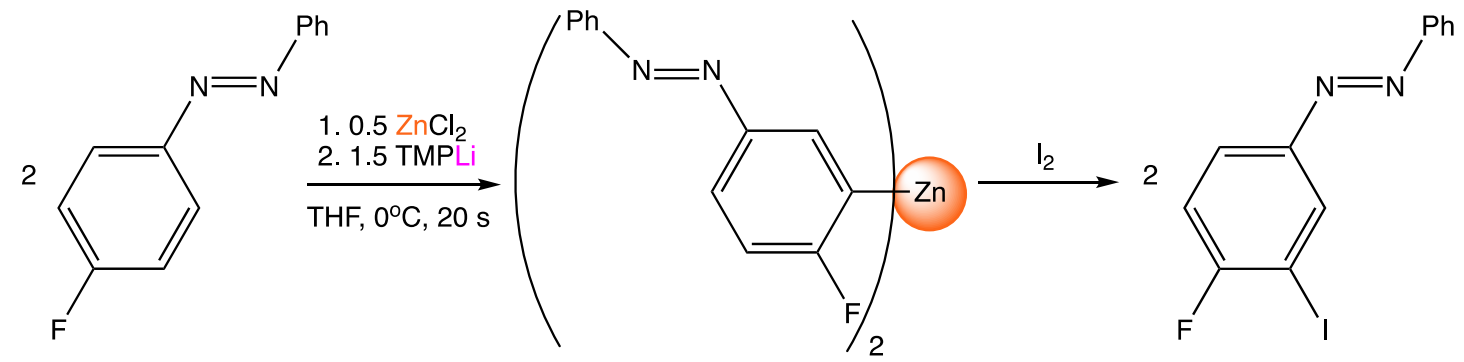

\subsubsection{Reactions Involving Direct Metal Insertion}

Studies probing the precise mechanistic role of lithium chloride in these turbo organometallic reagents have been rare. In an important exception, light has been shed on the role of the lithium salt during the insertion reaction between zinc powder and alkyl iodides. Thus, because of its excellent sensitivity fluorescence microscopy has allowed the detection of intermediates on the zinc surface. ${ }^{163}$ Several hypotheses were considered but the concluding one was that at certain reactive locations on the zinc surface oxidative addition takes place with the alkyl iodide producing organic intermediates "RZnl" that are transported from the surface to the THF solution by lithium chloride, forming a soluble "RZnl-LiCl" cocomplex. Concomitantly, this reexposes the most reactive sites on the zinc surface to more alkyl iodide enabling 
further oxidation addition reactions and repetition of the solubilizing process. Without the intervention by lithium chloride the organometallic intermediates would persist on the zinc surface.

For efficient atom economy, it is convenient to insert a metal directly into unsaturated organic halides for the preparation of organometallic reagents. An interesting development has been the use of the $\mathrm{C}_{60}$ fullerene as a catalyst (at $3 \mathrm{~mol}$ \% level) for inserting elemental magnesium into polycyclic aromatic halides, to produce Grignard reagents in good yield. ${ }^{164} \mathrm{C}_{60}$ is known to be a good electron transporter which is why it was chosen for this redox reaction. ${ }^{165}$ The synergistic aspect of this process is that a stoichiometric amount of $\mathrm{LiCl}$ needs to be present for efficient insertion with the salt assuming the role of a Lewis acid and "cleaner" to the electron rich magnesium metal surface. A similar method adding $\mathrm{ZnCl}_{2}$ to this mixture leads to arylzinc halide "ArZnX" reagents. Both the magnesium and zinc reagents can be reacted with common electrophiles such as those shown in Table 3.

Table 3. $\mathrm{C}_{60}$ fullerene catalyzed preparations of turbo-magnesium and turbo-zinc reagents and their application in electrophilic interception reactions. 


$$
\mathrm{Ar}-\mathrm{X} \stackrel{\substack{\mathrm{Mg}, \mathrm{nZnCl}, \mathrm{LiCl} \\ 3 \mathrm{~mol}_{\mathrm{O} \mathrm{C}_{60}}}}{\mathrm{DME}} \underset{\mathrm{Ar}-\mathrm{Ar}-\mathrm{Mg}-\mathrm{X}(\mathrm{n}=0)}{\longrightarrow} \stackrel{\mathrm{E}^{+}}{\longrightarrow} \mathrm{Ar}-\mathrm{E}
$$

(\%)

Turning back to elemental zinc, it was found to essentially not insert into ethyl 4iodobenzoate in THF solution after 24 hours. ${ }^{166}$ The key once again was to add LiCl. 
Introducing $\mathrm{LiCl}$ (150 mol\%) enabled the oxidative zinc insertion within 24 hours at 25 ${ }^{\circ} \mathrm{C}$ leading to the corresponding arylzinc halide reagent in $82 \%$ yield (Scheme 93 ).

Scheme 93. The beneficial effect of lithium chloride on reactions between zinc metal and ethyl 4-iodobenzoate.<smiles>CCOC(=O)c1ccc(I)cc1</smiles>

$$
\begin{aligned}
& \begin{array}{l}
\mathrm{Zn}(3 \text { equiv.) } \\
\mathrm{n} \mathrm{mol} \% \mathrm{LiCl} \\
\mathrm{x} \mathrm{mol} \% \operatorname{InCl}_{3}
\end{array} \\
& \mathrm{THF}
\end{aligned}
$$<smiles>CCOC(=O)c1ccc(-c2ccccc2)cc1</smiles>

\begin{tabular}{|c|c|c|c|c|}
\hline $\mathbf{n}$ & $\mathbf{x}$ & temp $\left({ }^{\circ} \mathbf{C}\right)$ & time (h) & yield (\%) \\
\hline 0 & 0 & 50 & 24 & $<5$ \\
\hline 150 & 0 & 25 & 24 & 82 \\
\hline 30 & 3 & 50 & 2 & 92 \\
\hline
\end{tabular}

The best conditions proved to be a catalytic recipe of $\mathrm{InCl}_{3}(3 \mathrm{~mol} \%)$ and $\mathrm{LiCl}(30 \mathrm{~mol} \%)$, which reduced the reaction time to 2 hours at $50{ }^{\circ} \mathrm{C}$ giving a yield of the arylzinc reagent of $92 \%$. It was found that using a THF/DMPU (1:1) mixture instead of neat THF speeds up the insertion. This $\mathrm{InCl}_{3} / \mathrm{LiCl}$ insertion method was applied to a long list of aryl and heteroaryl iodides, which in turn could be successfully trapped by electrophiles such as aryl or heteroaryl halides, allylic bromides and acid chlorides. For more challenging, less reactive aryl and heteroaryl bromides, $\ln (\mathrm{acac})_{3}$ proved to be a superior catalyst than $\mathrm{InCl}_{3}$ for the insertion step. This acac complex broadened the scope of the catalysis to enable the preparation of functionalized primary and secondary alkylzinc derivatives from the relevant alkyl bromides (Table 4).

Table 4. Representative insertion reactions of zinc into alkyl bromides and onward electrophilic interceptions of the organozinc intermediates. 


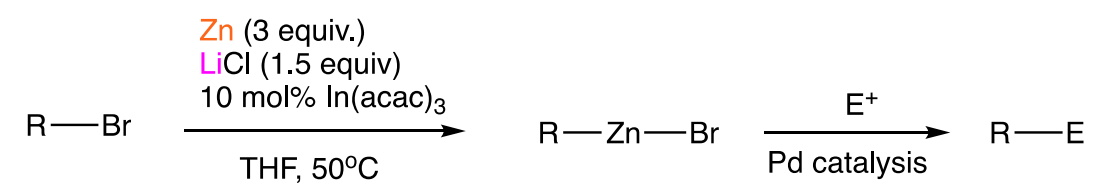

(a) electrophile

Lithium chloride mediation has also been an essential factor for the efficiency of the direct insertion of indium powder to cycloalkenyl iodides in THF. ${ }^{167}$ As Scheme 94 shows without $\mathrm{LiCl}$ the yield of the cycloalkenylindium (III) insertion product is very poor, but with LiCl the yield obtained after 12 hours increases to $78 \%$. To apply the highly functionalized cycloalkenylindium (III) reagents in Pd-catalyzed cross couplings with aryl bromides and iodides (4-iodoacetophenone in Scheme 94), the supernatant THF solution of the freshly prepared alkenylindium(III) reagents must be transferred 
to another dry and $\mathrm{N}_{2}$-flushed flask as any residual indium will lower the yield of the cross-coupled product. A benefit of this procedure is that in contrast to many metal insertions to alkenyl iodides which proceed with a loss of stereochemistry, inserting the synergistic indium/lithium chloride mixture to stereodefined (Z)- and (E)-styryl iodides in THF proceeded with high retention of stereochemistry. Following a Pdcatalyzed cross-coupling, a series of polyfunctionalized (Z)- and (E)-stilbenes were afforded with excellent stereoselectivity.

Scheme 94. Indium insertion reaction of 4-iodoacetophenone mediated by lithium chloride and subsequent cross-coupling application to produce 3-(4acetylphenyl)cyclohex-2-enone.
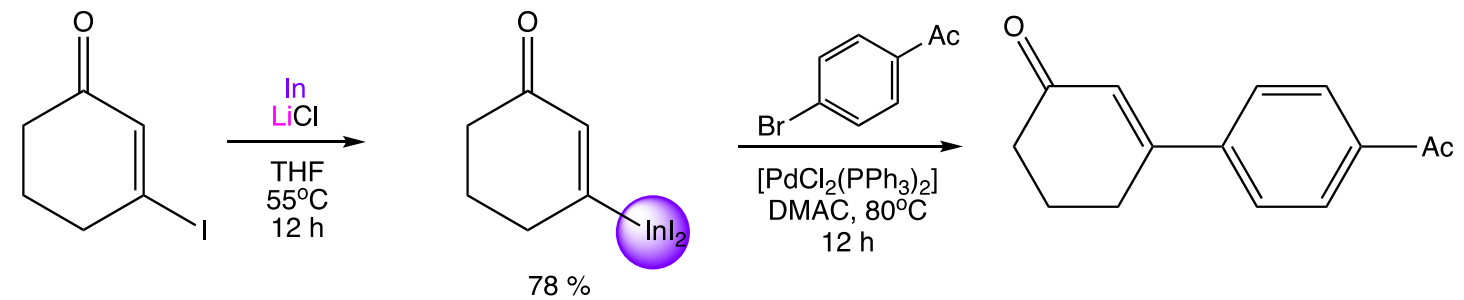

(<10\% without LiCl)

\subsubsection{Transition-Metal-Free Cross-Coupling Applications}

The versatility of lithium chloride supported reagents has also been extended to transition-metal-free cross-coupling chemistry. Benzylic zinc chlorides of general formula $\mathrm{RCH}_{2} \mathrm{ZnCl} \cdot \mathrm{LiCl}$ have been efficiently cross-coupled with substituted 4cyanopyridines in THF/DMPU solution under microwave irradiation $\left(40{ }^{\circ} \mathrm{C}, 0.5-1.5 \mathrm{~h}\right)$ to access functionalized 4-benzylated pyridines (Scheme 95). ${ }^{168}$ This selective benzylation procedure has also been applied successfully to polycyanoaromatic compounds under similarly mild conditions. 
Scheme 95. Microwave activated cross-coupling reactions of turbo benzylic zinc chloride intermediates with pyridine- and benzene-substituted cyanide complexes.<smiles>[R]c1cc(Cc2ccccc2)cc(Cc2ccc(F)cc2)n1</smiles><smiles></smiles>

The turbo-magnesium reagents TMPMgCl$\cdot \mathrm{LiCl}(104)$ and $\mathrm{R}_{2} \mathrm{NMgCl} \cdot \mathrm{LiCl}$ have also been used in the transition-metal-free process of aminating pyridine-2-sulfonamides and related nitrogen heterocycles. ${ }^{169}$ The former reagent executes ortho-magnesiation of the pyridine-2-sulfonamide, which following an electrophilic quench forms a 3substituted derivative. Desulfonylation of the sulfonamides is subsequently accomplished by applying the second magnesium amide reagent, where $\mathrm{R}_{2} \mathrm{~N}$ is derived from amines such as heterocyclic pyrrolidine or acyclic ${ }^{n} \mathrm{Bu}(\mathrm{Me}) \mathrm{NH}$. Two mechanistic possibilities were postulated (Scheme 96 ). The first starts by adding the magnesium amide to the pyridine leading to an intermediate, which, after $\mathrm{R}_{2} \mathrm{NSO}_{2} \mathrm{MgCl}$ elimination, affords an aminated pyridine (pathway A). In the second mechanism, $\mathrm{R}_{2} \mathrm{NMgCl} \cdot \mathrm{LiCl}$ addition to the sulfonamide group furnishes an intermediate, which may 
undergo an intramolecular transfer of the amino moiety leading to another intermediate and finally to the aminopyridine product (pathway B).

Scheme 96. Two proposed pathways for the transition-metal-free amination of pyridine-2-sulfonamides using the turbo-Hauser reagent $\mathrm{R}_{2} \mathrm{NMgCl} \cdot \mathrm{LiCl}$.

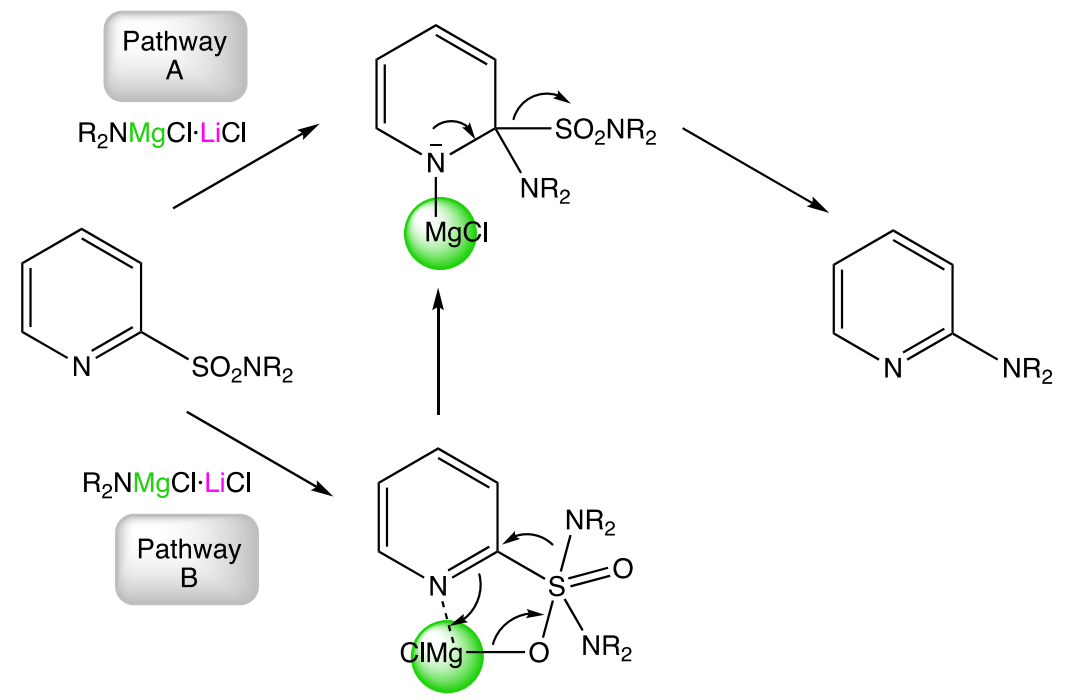

\subsection{Group 12 Chemistry}

\subsubsection{TMP-zincate and related metalating complexes}

Alkali metal zinc combinations have received considerable attention during this review period and examples of synergistic effects arising from this bimetallic partnership are plentiful. Alkali-metal-mediated zincation ( $\mathrm{AMMZn)}$, the origin and past examples of which have been reviewed elsewhere, ${ }^{50,51,170}$ has been particularly prominent. These are metalation reactions which conventional zinc reagents such as bis-alkyls cannot perform on their own, but when combined with alkali metal amides notably the bulky, strongly basic TMP amide, they can execute $\mathrm{Zn}-\mathrm{H}$ exchange on many aromatic and related substrates. To quote Robinson, "the TMP ligand has contributed to the renaissance of metalation chemistry involving organozincate compounds". ${ }^{171} \mathrm{~A}$ 
novel example is seen when $\mathrm{N}, \mathrm{N}$-diethyl-thiophene-2-carboxamide was subjected to TMP-zincate $\left[(\mathrm{TMEDA}) \mathrm{Na}(\mathrm{TMP})\left({ }^{t} \mathrm{Bu}\right) \mathrm{Zn}\left({ }^{t} \mathrm{Bu}\right)\right](\mathbf{1 2 3})$ in a 1:1 stoichiometry in benzene solution. Surprisingly the reaction was not a single-site $\mathrm{C}-\mathrm{H}$ to $\mathrm{C}-\mathrm{Zn}$ deprotonation as anticipated, but a 3,5-twofold deprotonation manifested in a molecular cage with a [16]crown-4 zincocyclic core in $\left[\left\{\mathrm{Na}\left[\mu-3,5-\left[2-\mathrm{C}(\mathrm{O}) \mathrm{NEt}_{2}\right]-\mathrm{C}_{4} \mathrm{H}_{1} \mathrm{~S}\right] \mathrm{Zn}\left({ }^{t} \mathrm{Bu}\right)\right\}_{4}\right](\mathbf{1 2 4}$, scheme 97)..$^{172}$

Scheme 97. Preparation and molecular structure of $\left[\left\{\mathrm{Na}\left[\mu-3,5-\left[2-\mathrm{C}(\mathrm{O}) \mathrm{NEt}_{2}\right]-\right.\right.\right.$ $\left.\left.\left.\mathrm{C}_{4} \mathrm{H}_{1} \mathrm{~S}\right] \mathrm{Zn}\left({ }^{t} \mathrm{Bu}\right)\right\}_{4}\right]$ (124), the dizincated product of $\mathrm{N}, \mathrm{N}$-diethyl-thiophene-2carboxamide.

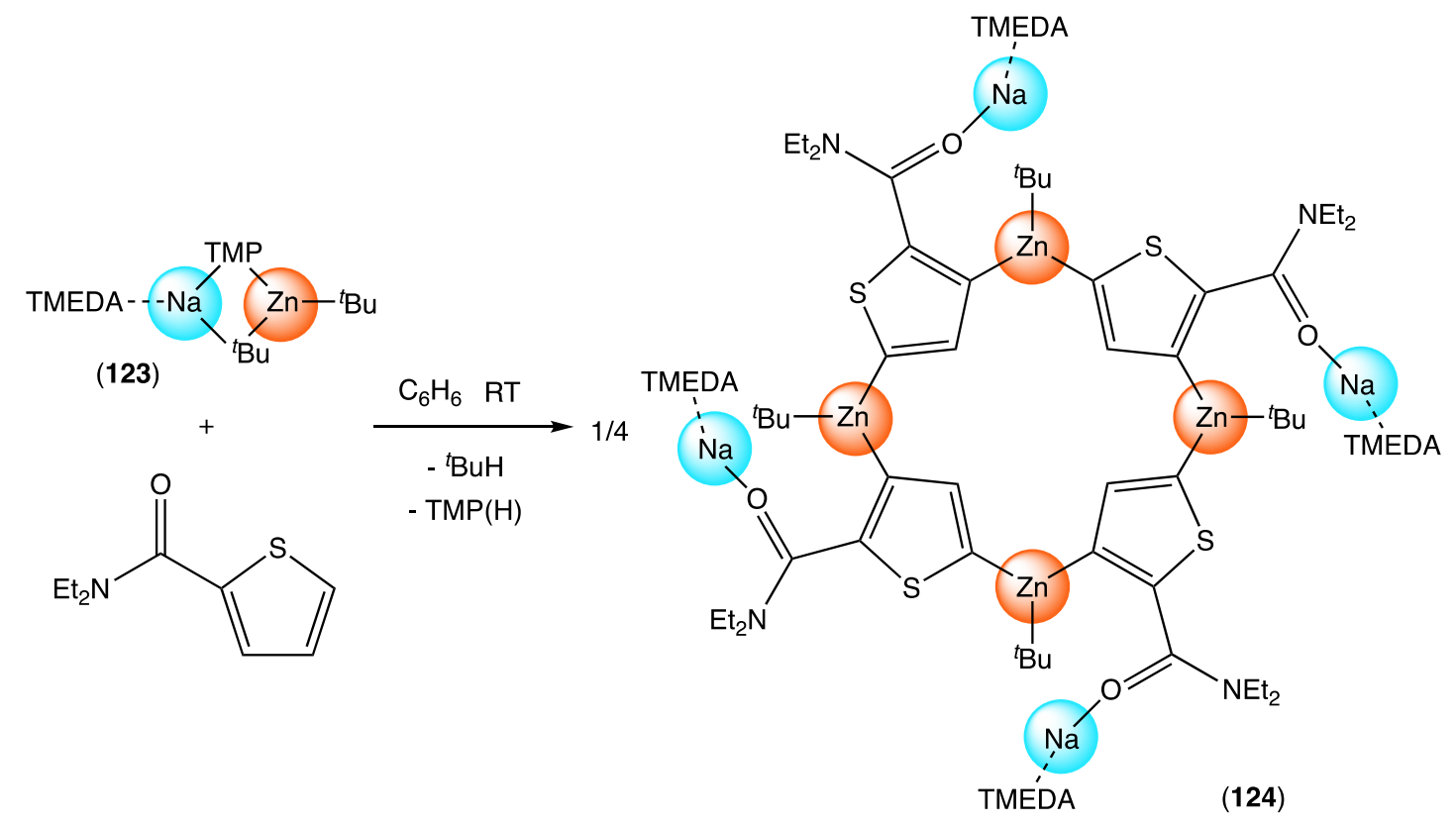

This cage was described as the first crystallographically characterized [16]metallacrown-4 complex of any metal where the non-metal atoms of the hexadecanuclear ring are exclusively carbon. TMP-zincate has also been applied to metallocene chemistry. Whereas ${ }^{t} \mathrm{Bu}_{2} \mathrm{Zn}$ is inert towards ferrocene, when dispensed in one or two molar equivalents TMP-zincate can monometalate or dizincate 
ferrocene to form mainly mono- or di-zincated complexes of formula [TMEDA $\left.\cdot \mathrm{Na}(\mathrm{TMP})\left\{\left(\mathrm{C}_{5} \mathrm{H}_{4}\right) \mathrm{Fe}\left(\mathrm{C}_{5} \mathrm{H}_{5}\right)\right\} \mathrm{Zn}{ }^{t} \mathrm{Bu}\right]$ or $\left[\left\{\mathrm{TMEDA} \cdot \mathrm{Na}(\mathrm{TMP}) \mathrm{Zn}\left({ }^{t} \mathrm{Bu}\right)\right\}_{2}\left(\mathrm{C}_{5} \mathrm{H}_{4}\right)_{2} \mathrm{Fe}\right](125$ and 126, scheme 98). ${ }^{173}$

Scheme 98. Products of mono (125, LHS) and di (126, RHS) TMP-zincation of ferrocene.

2

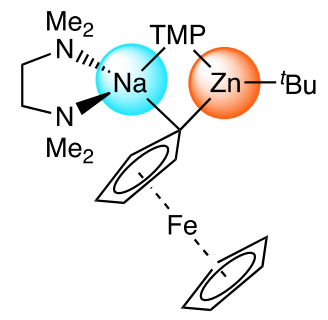

(125)

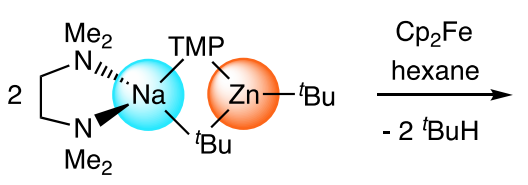

(123)

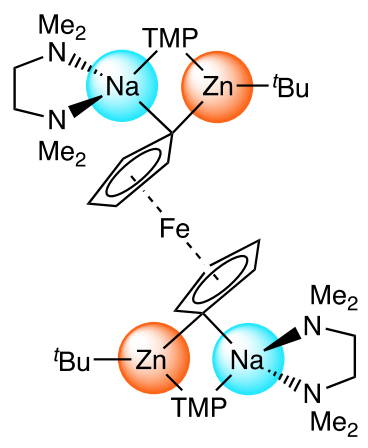

(126)

Note, that as described in section 2.5.3., a mixture of $\mathrm{Li}(T M P)$ and (TMP)Al'B $\mathrm{Bu}_{2}$ in the presence of THF can also synergistically mono- or di-metalate ferrocene but this is achieved in a two-step lithiation/trans-metal-trapping protocol, as opposed to the synchronized synergistic sodium-zinc protocol observed with TMP-zincate. Benzoylferrocene proved an interesting substrate for studying further the synergistic reactivity of $\mathbf{1 2 3}$ as it offers different reactivity sites. ${ }^{174}$ On its own the alkyl complex ${ }^{t} \mathrm{Bu}_{2} \mathrm{Zn}$ does not appear to react with any sites to any measurable extent. Remarkably the isolation of $\left[(T M E D A) \mathrm{Na}(\mu-\mathrm{TMP}) \mathrm{Zn}\left\{\mathrm{OC}\left({ }^{t} \mathrm{Bu}\right)(\mathrm{Ph})\left(\eta^{5}-\mathrm{C}_{5} \mathrm{H}_{3}\right) \mathrm{Fe}\left(\eta^{5}-\mathrm{C}_{5} \mathrm{H}_{5}\right)\right\}\right]$ established that the sodium zincate uses its two t-butyl substituents to react at two of these sites, namely to deprotonate the $\alpha$-position of the $C p$ ring, while at the same time adding across the carbonyl function in a 1,2-addition process (Scheme 99). 
Scheme 99. TMP zincation of benzoylferrocene with (RHS) and without (LHS) iodine interception showing both metalation and addition reactivity.

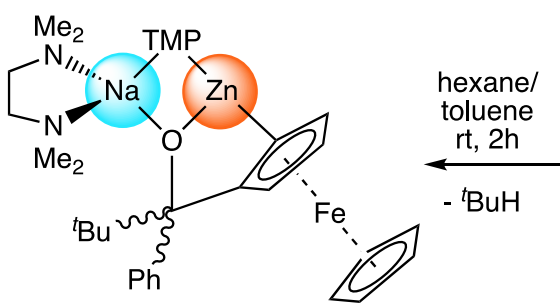

(127)

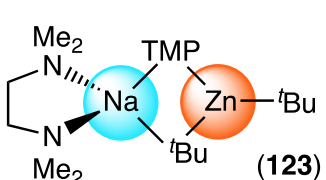

(123)

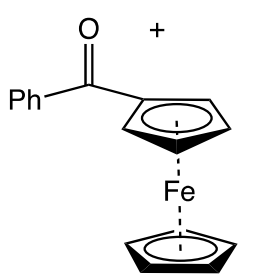

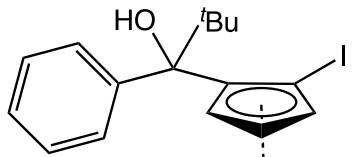

$\mathrm{Fe}$
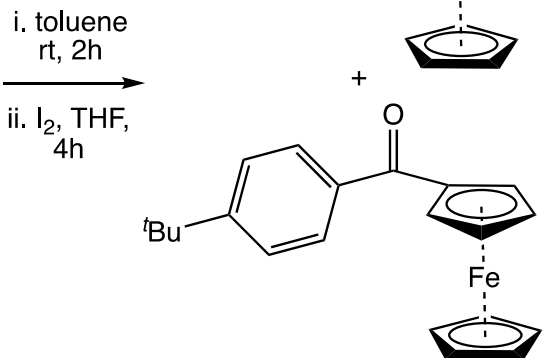

As this isolated product was obtained in a low yield the 1:1, benzoylferrocene: [(TMEDA) $\left.\mathrm{Na}(\mathrm{TMP})\left({ }^{t} \mathrm{Bu}\right) \mathrm{Zn}\left({ }^{t} \mathrm{Bu}\right)\right]$ reaction was treated with iodine in THF solution to give a mixture of two ferrocenyl derivatives, the expected product $\left[\mathrm{PhC}(\mathrm{OH})\left({ }^{t} \mathrm{Bu}\right)\left(\eta^{5}-\right.\right.$ $\left.\left.\mathrm{C}_{5} \mathrm{H}_{3} \mathrm{I}\right) \mathrm{Fe}\left(\eta^{5}-\mathrm{C}_{5} \mathrm{H}_{5}\right)\right]$ and the unexpected product $\left[4-{ }^{t} \mathrm{Bu}-\mathrm{C}_{6} \mathrm{H}_{4} \mathrm{C}(=\mathrm{O})\left(\eta^{5}-\mathrm{C}_{5} \mathrm{H}_{4}\right) \mathrm{Fe}\left(\eta^{5}-\right.\right.$ $\left.\mathrm{C}_{5} \mathrm{H}_{5}\right)$ ]. The latter product arises from a remote 1,6-addition across the phenyl ring, a reaction that was previously reported between this sodium zincate and benzophenone. ${ }^{175}$ "Cleave and capture chemistry" is a label given to examples of alkali-metal-mediated zincation and alkali-metal-mediated metalation in general where a bond in a substrate is deprotonated (cleaved) by the synergistic bimetallic base to generate an anion which is then captured by the residue of the bimetallic base. The rudiments of this idea have been summarized in a recent perspective article. ${ }^{176}$ An early exemplar is provided by the action of the (trimethylsilylmethyl)zincate $\left[(\mathrm{TMEDA}) \mathrm{Na}(\mathrm{TMP})\left(\mathrm{CH}_{2} \mathrm{SiMe}_{3}\right) \mathrm{Zn}\left(\mathrm{CH}_{2} \mathrm{SiMe}_{3}\right)\right]$ reagent (128) on THF. Conventional (nonsynergistic) bases such as $n$-butyllithium can readily deprotonate the cyclic ether THF at an $\alpha$ position, to store a lot of negative charge at the $\alpha$-carbon atom adjacent to the electron-rich $\mathrm{O}$ center, which in turn promotes a rapid ring opening that usually 
results in the formation of ethene and the enolate of acetaldehyde. ${ }^{177}$ In contrast the sodium zincate cleaves this same $\alpha-\mathrm{C}-\mathrm{H}$ bond but captures the THF $\left(\mathrm{C}_{4} \mathrm{H}_{7} \mathrm{O}^{-}\right)$anion intact in complex 129 (scheme 100). ${ }^{178}$

Scheme 100. Remarkable metalation of THF by sodium zincate reagent $\mathbf{1 2 8}$ in which the heterocyclic ring remains intact.

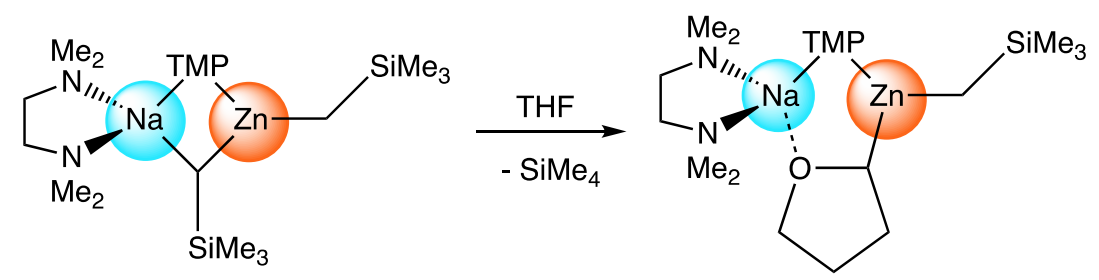

(128)

(129)

Since the sensitive THF anion is stabilized by coordination to both $\mathrm{Zn}$ and Na centers, through $C$ and $O$ centers respectively it can be utilized intact in a synthetic campaign. A more recent example of cleave and capture chemistry was observed with $N, N$ dimethylphenylethylamine, DMPEA, a member of an important class of pharmacological compound. ${ }^{179}$ Reaction with either [(TMEDA) $\left.\mathrm{Na}(\mathrm{TMP})\left({ }^{t} \mathrm{Bu}\right) \mathrm{Zn}\left({ }^{t} \mathrm{Bu}\right)\right]$ (123) or the related lithium zincate [(PMDETA)Li(TMP) $\left.\left({ }^{t} \mathrm{Bu}\right) \mathrm{Zn}\left({ }^{t} \mathrm{Bu}\right)\right](\mathbf{1 3 0})$ led to a $\beta$ elimination of an anionic $\mathrm{Me}_{2} \mathrm{~N}$ fragment of DMPEA that was captured in the crystalline products $\quad\left[(\mathrm{TMEDA}) \mathrm{Na}(\mathrm{TMP})\left(\mathrm{NMe}_{2}\right) \mathrm{Zn}\left({ }^{\mathrm{t}} \mathrm{Bu}\right)\right] \quad(\mathbf{1 3 1}) \quad$ and [(PMDETA $\left.) \mathrm{Li}\left(\mathrm{NMe}_{2}\right) \mathrm{Zn}\left({ }^{\mathrm{t}} \mathrm{Bu}\right)_{2}\right](\mathbf{1 3 2})$, respectively. In these two cases, it was assumed that the unobserved $\beta$-metalated intermediates are unstable and that the energy barriers to the $\alpha-\beta, \mathrm{Me}_{2} \mathrm{~N}-$ metal eliminations on forming the co-product styrene are small and easily overcome under the conditions employed.

Though as exemplified above the sodium zincate $\mathbf{1 2 3}$ usually functions as a base, it can also undergo single electron transfer (SET) processes. ${ }^{180}$ Its reaction with the 
stable nitroxy radical 2,2,6,6-tetramethylpiperidinyloxy (TEMPO) produces either [(TMEDA $\left.) \mathrm{Na}(\mu-\mathrm{TMP})\left(\mu-\mathrm{TEMPO}^{-}\right) \mathrm{Zn}\left({ }^{\mathrm{t}} \mathrm{Bu}\right)\right]$ (133) or $\left[\left(\mathrm{TMEDA}^{\mathrm{N}}\right) \mathrm{Na}\left(\mu-\mathrm{TEMPO}^{-}\right)_{2} \mathrm{Zn}\left({ }^{\mathrm{t}} \mathrm{Bu}\right)\right]$ (134) depending on the stoichiometry employed (Scheme 101).

Scheme 101. Three examples showing the single electron transfer reactivity of TMPzincate 123.

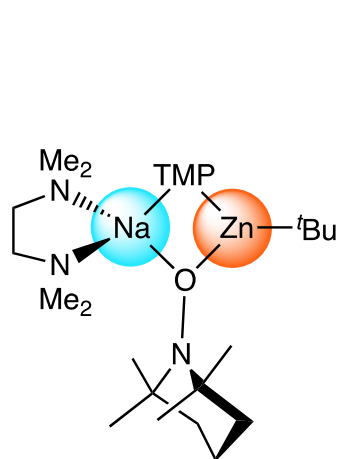

(133)
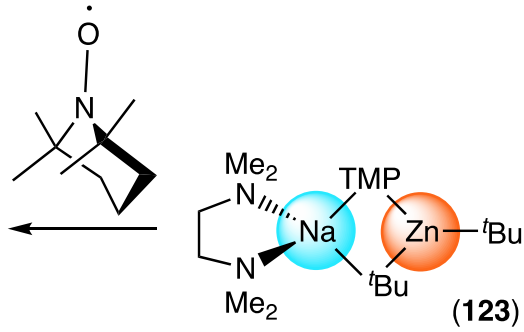

(123)<smiles>O=C(/C=C/c1ccccc1)c1ccccc1</smiles>
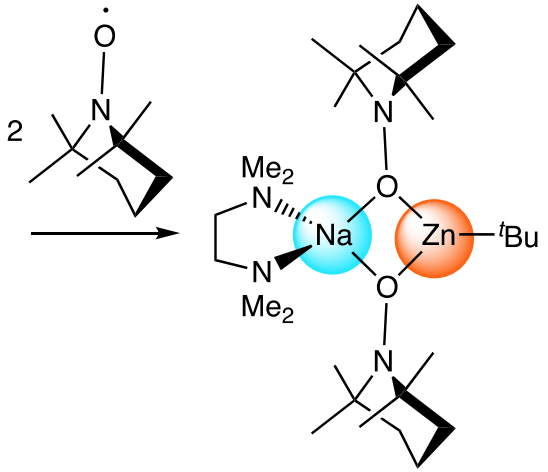

(134)

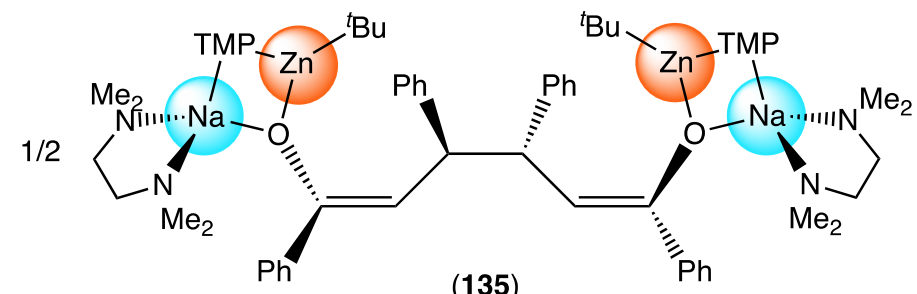

(135)

With the $\alpha, \beta$-unsaturated ketone, chalcone $[\mathrm{PhCOCH}=\mathrm{CHPh}]$, a $\mathrm{C}-\mathrm{C}$ bond formation takes place through two chalcone units coupling via their benzylic $C$ atoms to give $\left[\left\{(\mathrm{TMEDA}) \mathrm{Na}(\mu-\mathrm{TMP}) \mathrm{Zn}\left({ }^{\mathrm{t} B u}\right)\right\}_{2}(\mu-\mathrm{OCPhCH}=\mathrm{CHPhCHPhCH}=\mathrm{CPh}-\mu-\mathrm{O})\right] \quad$ (135), Scheme 101). Solvent choice can be a key factor in the outcome of the reactions of [(TMEDA) $\left.\mathrm{Na}(\mathrm{TMP})\left({ }^{t} \mathrm{Bu}\right) \mathrm{Zn}\left({ }^{t} \mathrm{Bu}\right)\right]$. In hexane solution at ambient temperature this synergistic sodium-zinc reagent selectively deprotonates $N$-Boc pyrrolidine at the position adjacent to the heteroatom (136, Scheme 102); whereas in toluene the generated $\alpha$-carbanion of the heterocycle attacks the solvent setting off a cascade of 
reactions that culminates in the formation of the pyrrolidine-substituted enolate $\left[\left\{(\mathrm{TMEDA}) \mathrm{Na}\left[\mathrm{OC}\left(\mathrm{NC}_{4} \mathrm{H}_{8}\right) \mathrm{CHPh}\right]\right\}_{2}\right](\mathbf{1 3 7}) \cdot{ }^{181} \mathrm{~A}$ mechanism for this cascade process has been postulated (Scheme 102).

Scheme 102. Solvent dependent reaction of TMP-zincate 123 and N-Boc pyrrolidine producing $\left[(\mathrm{TMEDA}) \mathrm{Na}(\mathrm{TMP})\left(\alpha-\mathrm{C}_{4} \mathrm{H}_{7} \mathrm{NBoc}\right) \mathrm{Zn}\left({ }^{t} \mathrm{Bu}\right)\right] \quad(\mathbf{1 3 6})$ in hexane and $\left[\left\{(\mathrm{TMEDA}) \mathrm{Na}\left[\mathrm{OC}\left(\mathrm{NC}_{4} \mathrm{H}_{8}\right) \mathrm{CHPh}\right]\right\}_{2}\right](137)$ in toluene.

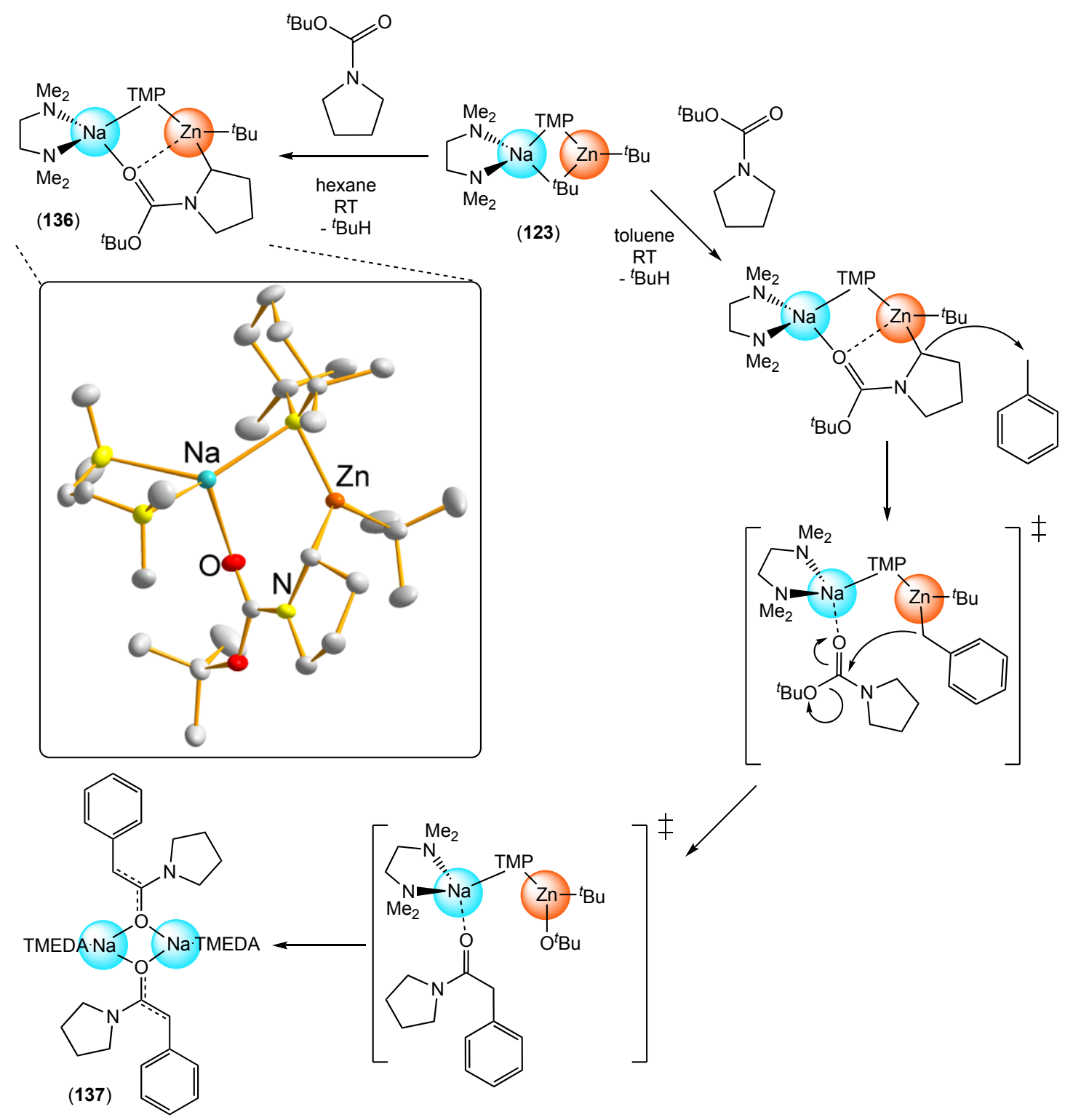


A black and white example of a bimetallic synergistic effect is evidenced in the formation of the mixed alkyl sodium zincate [(TMEDA)Na(TMP) $\left.\left({ }^{n} \mathrm{Bu}\right) \mathrm{Zn}\left({ }^{\mathrm{t}} \mathrm{Bu}\right)\right](138)$ from an equimolar mixture of $n$-butylsodium, di-tert-butylzinc, TMP(H) and TMEDA (Scheme 103). ${ }^{182}$ It may have been anticipated that the strong base $n$-butylsodium would have deprotonated TMP $(\mathrm{H})$ to generate the amide NaTMP and butane, and that the weak base di-tert-butylzinc would then simply cocomplex with NaTMP in the presence of TMEDA to produce the sodium homoalkylzincate 123. Instead it appears that a synergistic intermediate $\left[(T M E D A) \mathrm{Na}\left({ }^{n} \mathrm{Bu}\right)\left({ }^{t} \mathrm{Bu}\right) \mathrm{Zn}\left({ }^{t} \mathrm{Bu}\right)\right](\mathbf{1 3 9})$ is formed and that this reacts with $\operatorname{TMP}(\mathrm{H})$ through its most basic alkyl ${ }^{\text {t}} \mathrm{Bu}$ ligand (scheme 103).

Scheme 103. Unexpected formation of $\left[(T M E D A) N a(T M P)\left({ }^{n} B u\right) Z n\left({ }^{t} B u\right)\right]$ (138) via a twofold cocomplexation - deprotonation procedure.

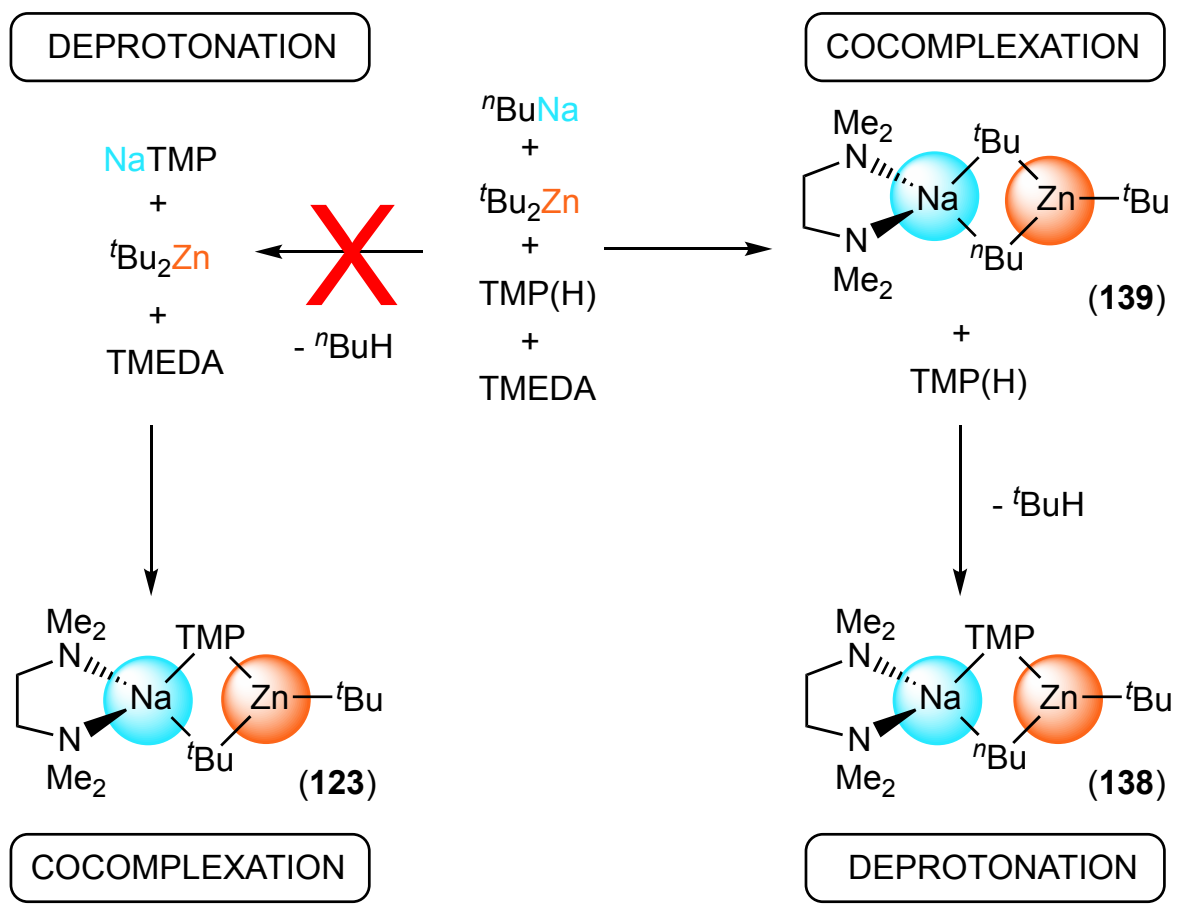

The reactivity of this heterotrileptic product $\mathbf{1 3 8}$ has been explored. It functions as a routine transamination agent when reacted with $\operatorname{HMDS}(\mathrm{H})$ or the chiral secondary 
amine $(+)$-bis $[(R)$-1-phenylethyl]amine $[\operatorname{PEA}(H)]$ with retention of the bimetallic scaffold in 140 and 141 respectively, but breaks down to separated homometallic sodium and zinc species when deprotonating the bulky amine $N$-phenylnaphthalen-1amine $[\mathrm{PNA}(\mathrm{H})]$ (Scheme 104).

Scheme 104. Divergent reactivity of $\left[(T M E D A) \mathrm{Na}(T M P)\left({ }^{n} \mathrm{Bu}\right) \mathrm{Zn}\left({ }^{t} \mathrm{Bu}\right)\right]$ (138) with secondary amines.
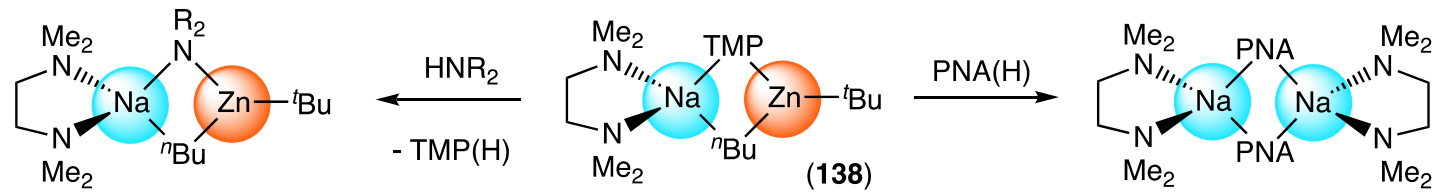

$\mathrm{R}=\mathrm{SiMe}_{3}(\mathbf{1 4 0})$ $\mathrm{CH}(\mathrm{Me}) \mathrm{Ph}(\mathbf{1 4 1})$

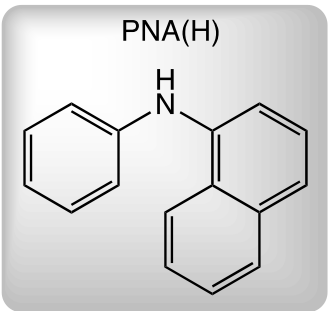
$+\operatorname{TMP}(\mathrm{H})$

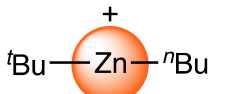

Strangely, [(TMEDA) $\mathrm{Na}(\mathrm{TMP})\left({ }^{\mathrm{n} B \mathrm{Bu}} \mathrm{Zn}\left({ }^{\mathrm{t}} \mathrm{Bu}\right)\right]$ failed to react with toluene, anisole, or $N$, $N$-dimethylbenzamide, marking it as a much less efficient base than its versatile di- $t$ butyl variant $\left[(T M E D A) \mathrm{Na}(\mathrm{TMP})\left({ }^{t} \mathrm{Bu}\right) \mathrm{Zn}\left({ }^{t} \mathrm{Bu}\right)\right]$, though oddly it does doubly deprotonate benzene to form the known arenediide complex [(TMEDA)Na(TMP) $\left.\left({ }^{t} \mathrm{Bu}\right) \mathrm{Zn}\left(\mathrm{C}_{6} \mathrm{H}_{4}\right) \mathrm{Zn}\left({ }^{t} \mathrm{Bu}\right)(\mathrm{TMP}) \mathrm{Na}(\mathrm{TMEDA})\right]$ (142). ${ }^{183}$ Another exemplar of a successful synergistic zincate reaction is witnessed with pyrazine. ${ }^{184}$ On treatment with ${ }^{t} \mathrm{Bu} u_{2} \mathrm{Zn}$, only the donor-acceptor complex $\left[\left\{\mathrm{Zn}^{t} \mathrm{Bu}_{2}\right\}_{3}\left\{\mathrm{C}_{4} \mathrm{H}_{4} \mathrm{~N}_{2}\right\}_{4}\right]$ (143) forms having an unusual triangular trinuclear arrangement. In contrast, when the dialkylzinc molecule is part of the lithium amidozincate [(THF)Li(TMP) $\left.\left({ }^{t} \mathrm{Bu}\right) \mathrm{Zn}\left({ }^{\mathrm{t} B u}\right)\right](\mathbf{1 4 4})$, pyrazine is deprotonated twice at the 2,5-positions to generate the isolable bimetallic intermediate $\left.\left[2,5-\left\{(\mathrm{THF})_{2} \mathrm{LiZn}(\mathrm{TMP})^{t} \mathrm{Bu}\right)\right\}_{2}\left(\mathrm{C}_{4} \mathrm{H}_{2} \mathrm{~N}_{2}\right)\right]$ (145), which provides access to 
2,5-diiodopyrazine on treatment with iodine. On changing the zincate reagent to the homoleptic alkyl zincate [(PMDETA)LiZn $\left.{ }^{t} \mathrm{Bu}_{3}\right](\mathbf{1 4 6})$ a 1,2-addition reaction takes place to afford [(PMDETA)Li $\left.\left\{\mathrm{C}_{4} \mathrm{~N}_{2} \mathrm{H}_{4}\left({ }^{t} \mathrm{Bu}\right)\right\} \mathrm{Zn}^{t} \mathrm{Bu}_{2}\right]$ (147), which forms 2-t-butylpyrazine following hydrolysis and aerobic oxidation (scheme 105).

Scheme 105. Zincate dependent reactions of pyrazine, showing addition or dimetalation.

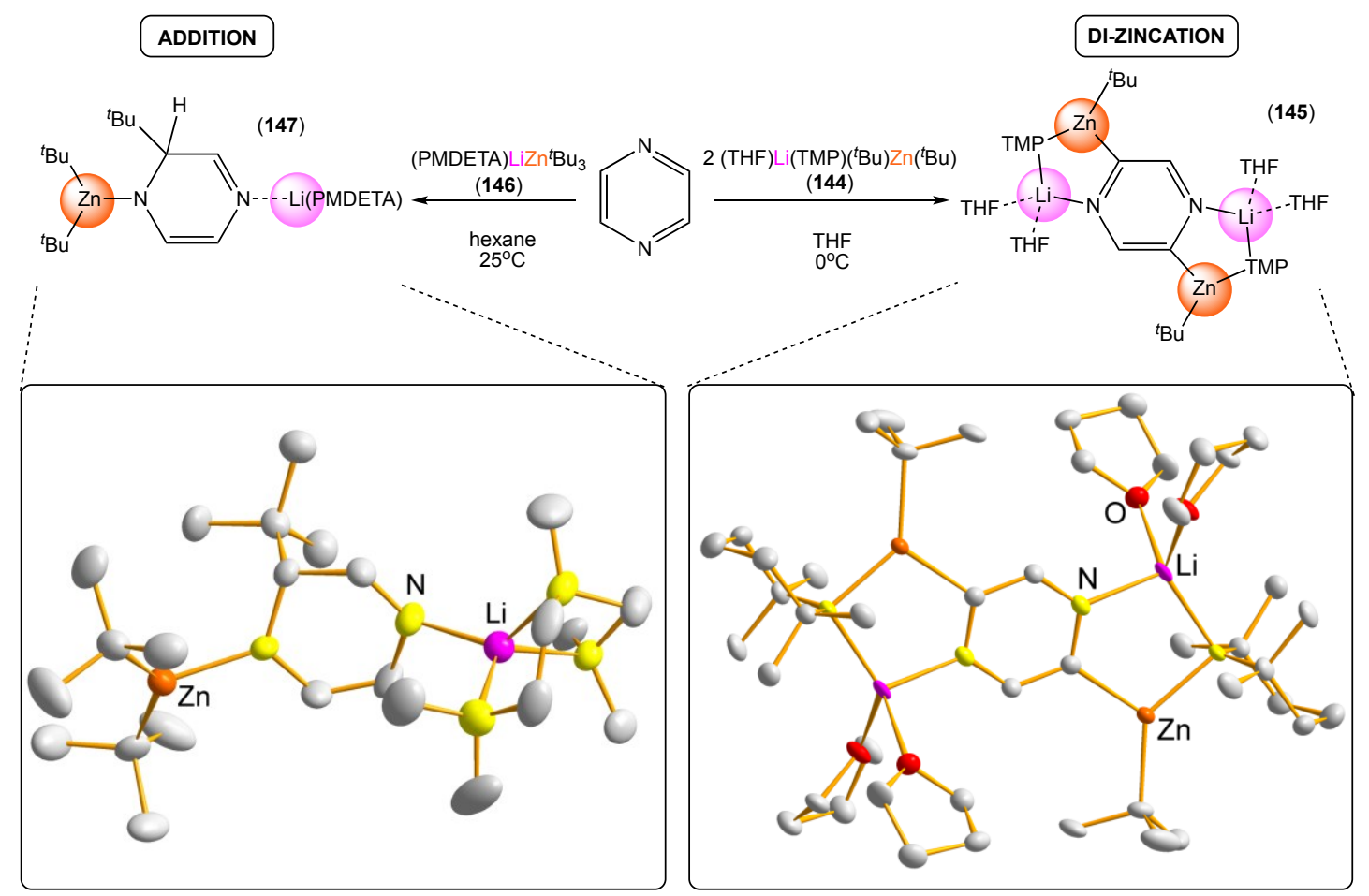

A synergistic mixture of LiTMP and $\mathrm{ZnCl}_{2} \cdot$ TMEDA in a 3:1 stoichiometric ratio has been used to metalate a series of biaryl substrates in THF at room temperature. ${ }^{185}$ Operating in a stepwise manner, that is lithiation followed by stabilisation with $\mathrm{Zn}$ species, this particular mixture proved successful and applicable to an impressive scope of aromatic substrates including ketones, ${ }^{186,187} 2$-arylquinolines, ${ }^{188}$ azoles, ${ }^{189-192}$ $\mathrm{N}$-arylated pyrroles and indoles, ${ }^{193}$ as well as $\mathrm{N}, \mathrm{N}$-dialkylferrocenecarboxamides (Table 5). ${ }^{194}$ 
Table 5. Representative synergistic zincations of various aromatic and heteroaromatic substrates showing their products following iodolysis.

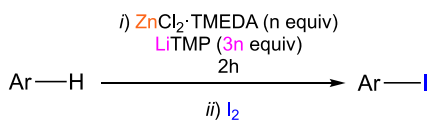

\begin{tabular}{|c|c|c|c|c|c|c|c|c|c|c|c|}
\hline product & $n$ & $\begin{array}{l}\text { temp } \\
\left({ }^{\circ} \mathrm{C}\right)\end{array}$ & $\begin{array}{l}\text { yield } \\
(\%)\end{array}$ & product & $n$ & $\begin{array}{l}\text { temp } \\
\left({ }^{\circ} \mathrm{C}\right)\end{array}$ & $\begin{array}{l}\text { yield } \\
(\%)\end{array}$ & product & $n$ & $\begin{array}{l}\text { temp } \\
\left({ }^{\circ} \mathrm{C}\right)\end{array}$ & $\begin{array}{l}\text { yield } \\
(\%)\end{array}$ \\
\hline & 1 & $\mathrm{rt}$ & 79 & & 0.75 & $\mathrm{rt}$ & 86 & & 1 & $\mathrm{rt}$ & 91 \\
\hline & 1 & $\mathrm{rt}$ & 92 & & 0.5 & it & 97 & & 0.5 & it & 61 \\
\hline & 0.75 & $\mathrm{rt}$ & 92 & & 0.75 & $\mathrm{rt}$ & 89 & & 0.5 & $\mathrm{rt}$ & 74 \\
\hline & 1 & $\mathrm{rt}$ & 82 & & 1.5 & -55 & 60 & & 1 & -55 & 80 \\
\hline & 1.5 & $\mathrm{rt}$ & 85 & & 0.5 & 0 & 78 & & 1 & -55 & 27 \\
\hline
\end{tabular}

As expected in the case of aromatic ketones ${ }^{186,195}$ the metalation took place at the position adjacent to the carbonyl group, with pyridyl ketones being functionalized on the pyridyl ring. ${ }^{187}$ It was noted that in the case of benzophenone and fluorenone, the synergistic lithium - zinc mixture was not appropriate but could be replaced by a congeneric lithium - cadmium mixture to obtain the desired product in good yields. ${ }^{186}$ With $\mathrm{N}$-phenylpyrrole and -indole, the metalations were carried out in hexane solution containing TMEDA which regioselectively afforded the 2-iodo derivatives 
after subsequent iodolysis. With pyrroles and indoles bearing $\mathrm{N}$-substituents such as 2-thienyl, 3-pyridyl, 4-methoxyphenyl and 4-bromophenyl, the reactions all took place on the substituent, at the position either adjacent to the heteroatom $(\mathrm{S}, \mathrm{N})$ or ortho to the heteroatom-containing substituent (OMe, $\mathrm{Br}) \cdot{ }^{193}$ Similar outcomes were noted in the study with 2-substituted quinolines where in the case of 2-phenyl and 2naphthylquinoline the reaction took place at the 8-position of the quinoline ring, but in the case of 3-pyridyl, 2-furyl, or 2-thienyl substituents the metalation preferentially occurred on the substituent heterocycle at the position adjacent to the heteroatom. ${ }^{188}$ The preference to metalate 2 -thienyl substituent allowed the authors to functionalize 1-(2-thienyl)-1H-indazole and 1-(2-thienyl)-1H-benzotriazole (again at the position adjacent to the S-atom). ${ }^{190}$ Other 1 -aryl- $1 H$-indazoles underwent ring opening upon deprotonation of the indazole ring. In the series of $N$-phenyltriazoles ${ }^{189-191}$ and $N$-phenylpyrazole ${ }^{191}$ a mixture of products was obtained. However, upon increasing the amount of base it was possible to better control the outcome and diiodinated products were isolated in high yields. The same mixture was used to metalate chiral ferrocenecarboxylates which after iodolysis afforded products in moderate to good yields and diastereoselectivities. Interestingly, it was found that the reverse sequential addition of organometallic reagents, that is first adding $\mathrm{Zn}(\mathrm{TMP})_{2}$ or even $\mathrm{ZnCl}_{2}$ to the substrate followed by the addition of LiTMP, induced an increase in diastereoselectivity. This observation was then used in a protocol of double asymmetric induction when commercial $(R)$ - and $(S)$ - bis[1-phenylethyl]amine] $[(\operatorname{PEA}(H)]$ were used instead of TMP(H). In this case, although $(S)-P E A(H)$ afforded low yields and selectivities, $(R)-P E A(H)$ proved efficient providing the $S$ enantiomer in $85 \%$ yield and 91 de. ${ }^{196}$ Utilising a bis[(S)-1-phenylethyl]amido-based lithium - zinc 
combination to metalate $\mathrm{N}, \mathrm{N}$-diisopropylferrocenecarboxamide afforded high yields but low enantioselectivities. ${ }^{197}$ Changing their approach, the authors tested a series of dialkyl bis[(S)-1-phenylethyl]amido-based lithium and dilithium zincates and found that using 2 equivalents of $\mathrm{Me}_{2}(\mathrm{PEA})_{2} \mathrm{ZnLi} 2$ at $0{ }^{\circ} \mathrm{C}$ can maintain the high yield, but increase the ee to $86 \%$ in favor of the $S$-enantiomer.

\subsubsection{Uses in $\mathbf{N}$-Heterocyclic Carbene (NHC) Chemistry}

Lithium - zinc synergistic effects have also realized some "firsts" in $\mathrm{N}$-heterocyclic carbene $(\mathrm{NHC})$ chemistry. Previously reported zinc $\mathrm{NHC}$ complexes have been neutral and C2 (normal site) based. However, a lithium anionic N-heterocyclic dicarbene proved a convenient platform for the formation of the first charged NHC-based triorganozincates. ${ }^{171}$ Depending on the reaction stoichiometry, solvents and conditions employed, its reaction with diethylzinc produced triorganozincates with 1:3 or 1:1 Li:Zn stoichiometric ratios in which at least one $\mathrm{Zn}$ atom occupied the $\mathrm{C} 4$ (abnormal) site (148 and 149, Scheme 106).

Scheme 106. Solvent dependent zincation reactions of NHCs, where $\mathrm{R}=2,6-{ }^{i} \mathrm{Pr}_{2} \mathrm{C}_{6} \mathrm{H}_{3}$. 

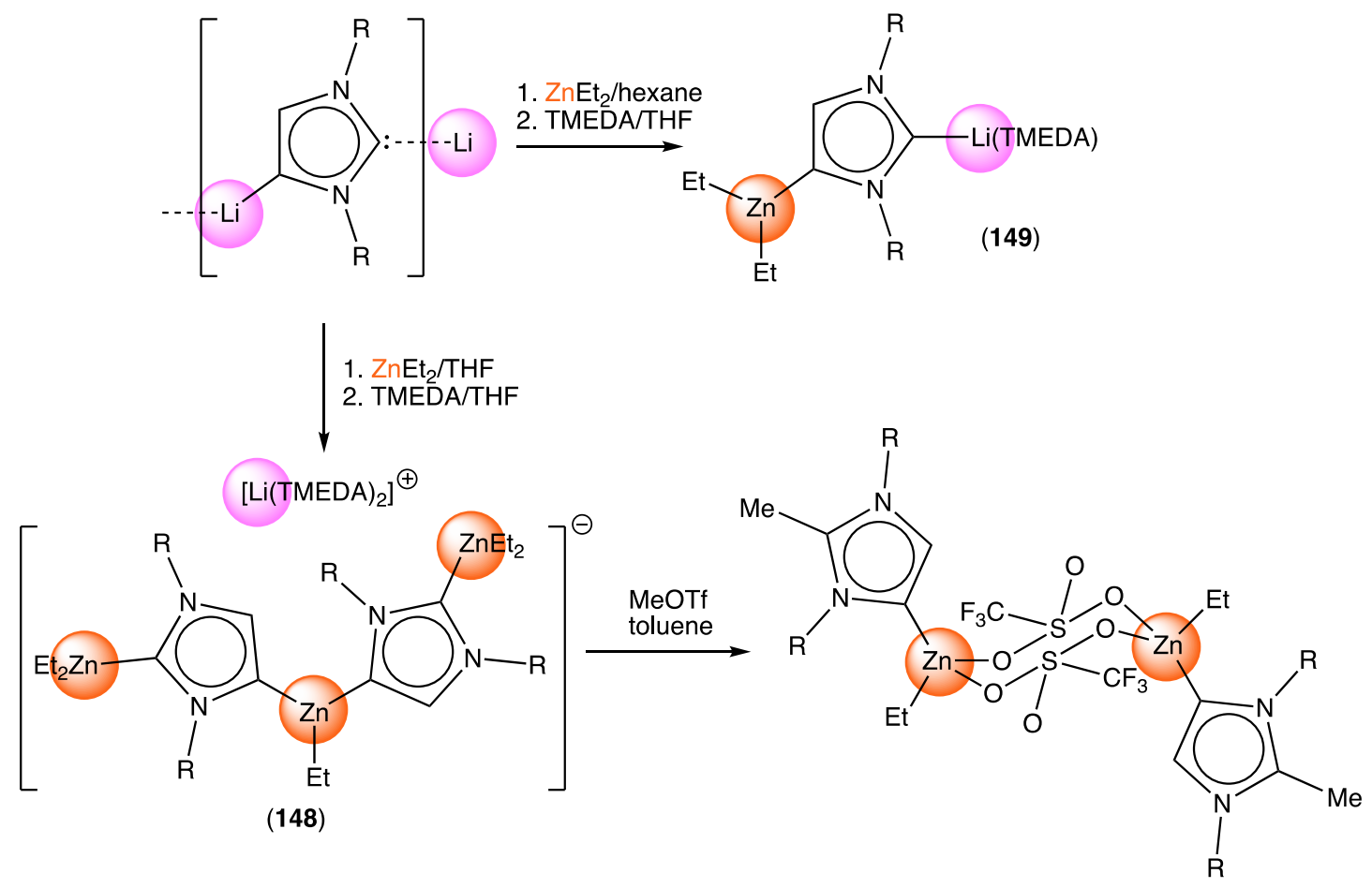

On reaction with MeOTf, the 1:3 triorganozincate complex 148 allowed access to the first aNHC-based all-zinc complex. Sodium-mediated zincation chemistry has also made an impact in NHC chemistry. ${ }^{198}$ Direct $\mathrm{C}-\mathrm{H}$ to $\mathrm{C}-\mathrm{Zn}$ metalation of the same NHC $\operatorname{IPr} \quad[=$ 1,3-bis(2,6-di-isopropylphenyl)imidazol-2-ylidene $]$ occurs with $\left[(\mathrm{TMEDA}) \mathrm{Na}(\mathrm{TMP})\left({ }^{t} \mathrm{Bu}\right) \mathrm{Zn}\left({ }^{t} \mathrm{Bu}\right)\right] \quad(\mathbf{1 2 3})$ to afford $\quad(\mathrm{THF})_{3} \mathrm{Na}[: \mathrm{C}\{[\mathrm{N}(2,6-$ $\left.\left.\left.\left.{ }^{i} \mathrm{Pr}_{2} \mathrm{C}_{6} \mathrm{H}_{3}\right)\right]_{2} \mathrm{CHCZn}\left({ }^{t} \mathrm{Bu}_{2}\right)\right\}\right]$ (150), which in turn functions as an alkenyl transfer agent with the gold(I) complex $\left[\mathrm{ClAu}\left(\mathrm{PPh}_{3}\right)\right]$ to produce the digold complex $[\mathrm{ClAu}: \mathrm{C}\{[\mathrm{N}(2,6-$ $\left.\left.\left.\left.{ }^{i} \mathrm{Pr}_{2} \mathrm{C}_{6} \mathrm{H}_{3}\right)\right]_{2} \mathrm{CHCAu}\left(\mathrm{PPh}_{3}\right)\right\}\right](\mathbf{1 5 1}$, scheme 107). The latter process is a rare example of a double transmetalation reaction, which involves the exchange of both cationic $\mathrm{Na}^{+}$ and neutral ${ }^{t} \mathrm{Bu}_{2} \mathrm{Zn}$ by gold entities on the NHC scaffold. In contrast, when IPr is mixed with ${ }^{t} \mathrm{Bu}_{2} \mathrm{Zn}$ on its own, only the simple donor-acceptor complex $(\operatorname{IPr}) \mathrm{Zn}^{t} \mathrm{Bu}_{2}$ (152) forms, which in turn can co-complex with $t$-butyllithium to produce $\left[(\operatorname{Ir})\left(\operatorname{LiZn}^{t} \mathrm{Bu}_{3}\right]\right.$ (153, scheme 107). 
Scheme 107. Reactions of the $N$-heterocyclic carbene IPr under synergistic (top) and non-synergistic (bottom) conditions. The zincate product (150) of the former acts as an alkenyl transfer agent to afford digold(I) complex 151, the structure of which is shown in the inset.

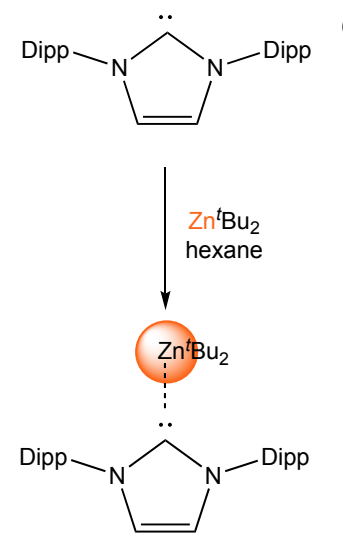

(152)
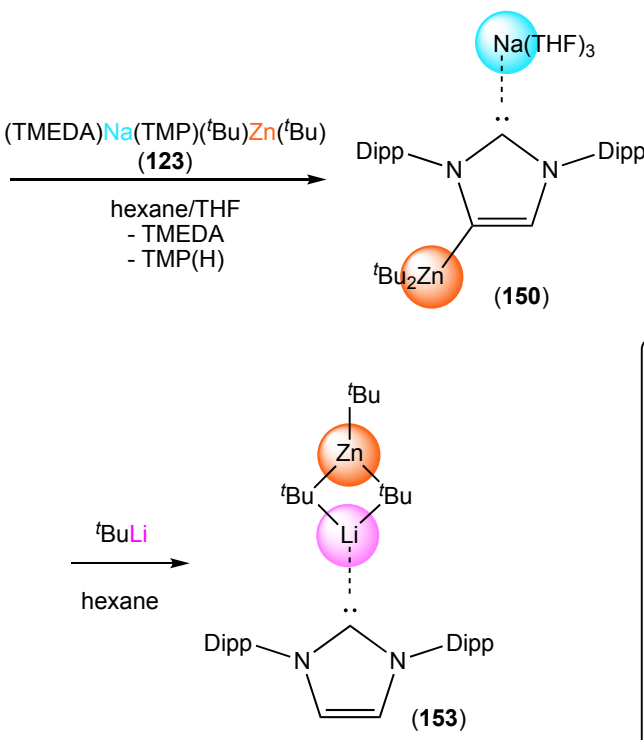
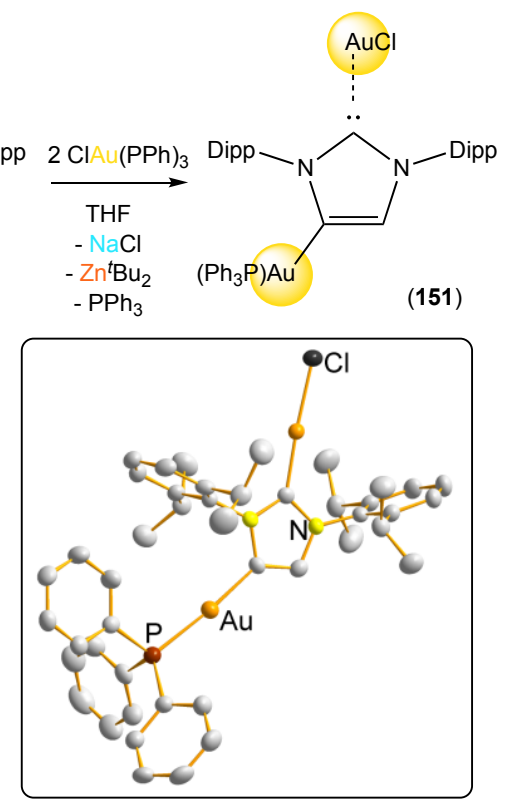

Reaction of the ditopic carbanionic carbene potassium $\operatorname{IPr}[: \mathrm{C}[\mathrm{N}(2,6-$ $\left.\left.\left.{ }^{i} \mathrm{Pr}_{2} \mathrm{C}_{6} \mathrm{H}_{3}\right)\right]_{2}(\mathrm{CH}) \mathrm{CK}(\mathrm{THF})_{2}\right]$ with the zinc amide $\mathrm{Zn}\left[\mathrm{N}\left(\mathrm{SiMe}_{3}\right)_{2}\right]_{2}$ in a $1: 1$ stoichiometric ratio in THF solution produces the novel three-coordinate zincate complex $[\mathrm{K}(2,2,2-$ crypt $)]^{+}\left[\mathrm{Zn}\left\{\mathrm{C}(\mathrm{CH})\left[\mathrm{N}\left(2,6^{-}{ }^{i} \mathrm{Pr}_{2} \mathrm{C}_{6} \mathrm{H}_{3}\right)\right]_{2} \mathrm{C}:\right\}\left\{\mathrm{N}\left(\mathrm{SiMe}_{3}\right)_{2}\right\}_{2}\right]^{-}\left(\mathbf{1 5 4}\right.$, Scheme 108). ${ }^{199}$ This can be considered a cocomplexation reaction with the added factor that the cryptand ligand has sequestered the $\mathrm{K}$ center from the carbene, leaving the zinc center to occupy the abnormal C4 site of the NHC.

Scheme 108. Cocomplexation of a potassium abnormal carbanionic NHC with a zinc bis(amide) in the presence of the $\mathrm{K}^{+}$sequestering agent 2,2,2-crypt, yielding 154 . 

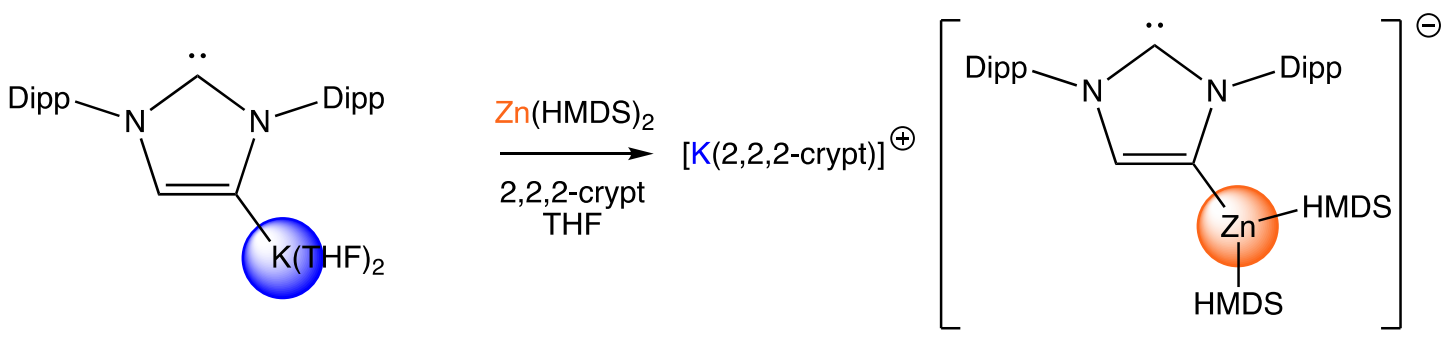

(154)

\subsubsection{Special Cocomplexation Chemistry of Zincates}

Whereas a good proportion of the alkali metal - zinc synergistic reactivity has involved the TMP ligand, one recent study 200 has focused on the smaller cis-2,6dimethylpiperidide ligand. Made from three commercially available components via cocomplexation, the DMP-zincate [(TMEDA)LiZn(cis-DMP)Et ${ }_{2}$ ] (155, Scheme 109), adopts a contacted ion pair structure of a type common in zincate chemistry. Control deprotonation reactions with $N, N$-diisopropylbenzamide show the synergistic lithium-diethylzincate base can outperform its di-tert-butyl congener and a related HMDS complex, is competitive with a related diisopropylamide complex, but is slightly less efficient than a related TMP system.

Scheme 109. Preparation of DMP-zincate [(TMEDA)LiZn(cis-DMP)Et $\left.{ }_{2}\right](\mathbf{1 5 5})$ and its reactivity towards $\mathrm{N}, \mathrm{N}$-diisopropylbenzamide compared to that of other amidozincate complexes. 


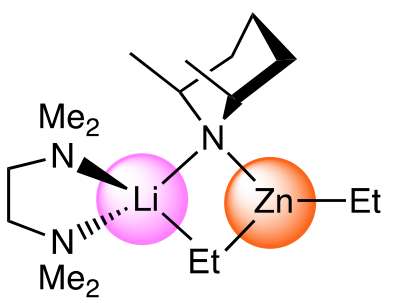

(155)

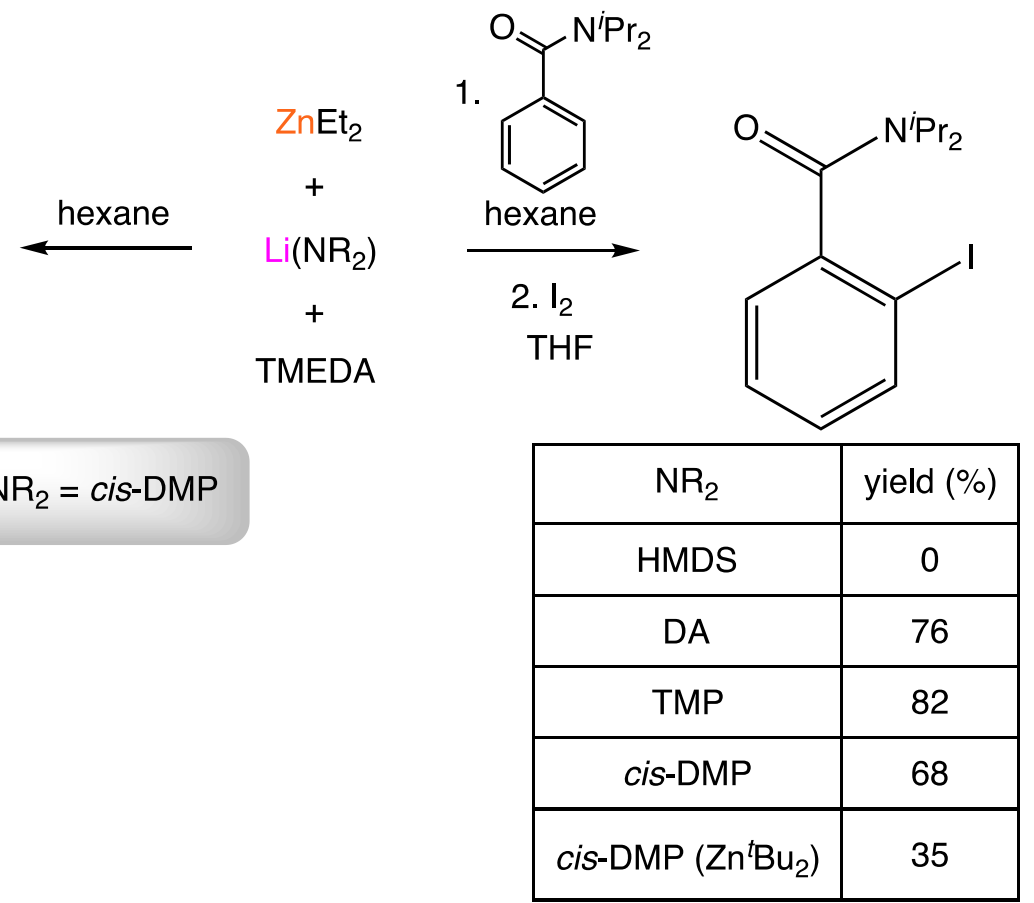

Cocomplexation of different amounts of their monometallic components PhLi and $\mathrm{Ph}_{2} \mathrm{Zn}$, have produced the lithium homoarylzincate reagents [ $\mathrm{LiZnPh} \mathrm{H}_{3}$ ] $(\mathbf{1 5 6}$, first made by Wittig) ${ }^{26}$ and $\left[\mathrm{Li}_{2} \mathrm{ZnPh}_{4}\right](\mathbf{1 5 7})$, that have been characterized by NMR studies with the former also crystallographically characterized in the form of the ether solvate $\left[\mathrm{LiZnPh}_{3}\left(\mathrm{O}^{n} \mathrm{Bu}_{2}\right)_{2}\right]\left(\mathbf{1 5 8}\right.$, Figure 28). ${ }^{201}$

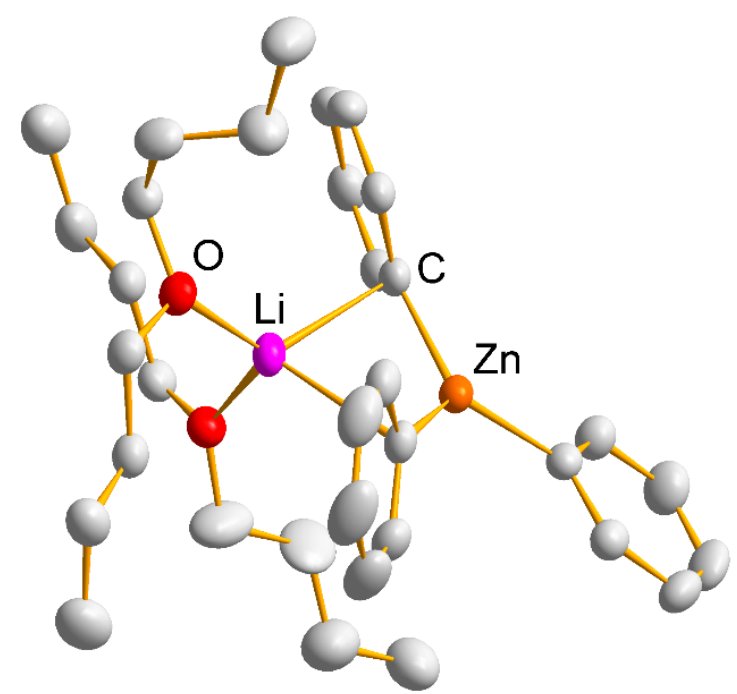


Figure 28. Molecular structure of $\left[\mathrm{LiZnPh}_{3}\left(\mathrm{O}^{n} \mathrm{Bu}_{2}\right)_{2}\right](\mathbf{1 5 8})$, the etherate solvate of the historically important zincate 156.

Earlier work established that the neutral arylzinc compound $\mathrm{Ph}_{2} \mathrm{Zn}$ is unable to transfer one of its phenyl groups to acridine in the absence of a transition-metal catalyst and harsh reaction conditions $\left(130{ }^{\circ} \mathrm{C}, 20\right.$ h)..$^{202}$ In contrast, both polyphenylzincate derivatives 156 and 157 were found to synergistically chemoselectively arylate acridine at the 9 position, with the optimum conditions of $125^{\circ} \mathrm{C}$, in THF solution for 20 minutes under microwave irradiation. The higher order (2Li:1Zn) zincate 157 was more effective than its lower order (1Li:1Zn) counterpart 156 (95\% versus $70 \%$ ), whereas without alkali metal mediation $\mathrm{Ph}_{2} \mathrm{Zn}$ was completely inert under the same conditions (Scheme 110).

Scheme 110. Chemoselective arylation of acridine by synergistic lithium triphenyl-and tetraphenyl-zincates $156(x=1)$ and $157(x=2)$, showing their superiority over nonsynergistic diphenylzinc. 

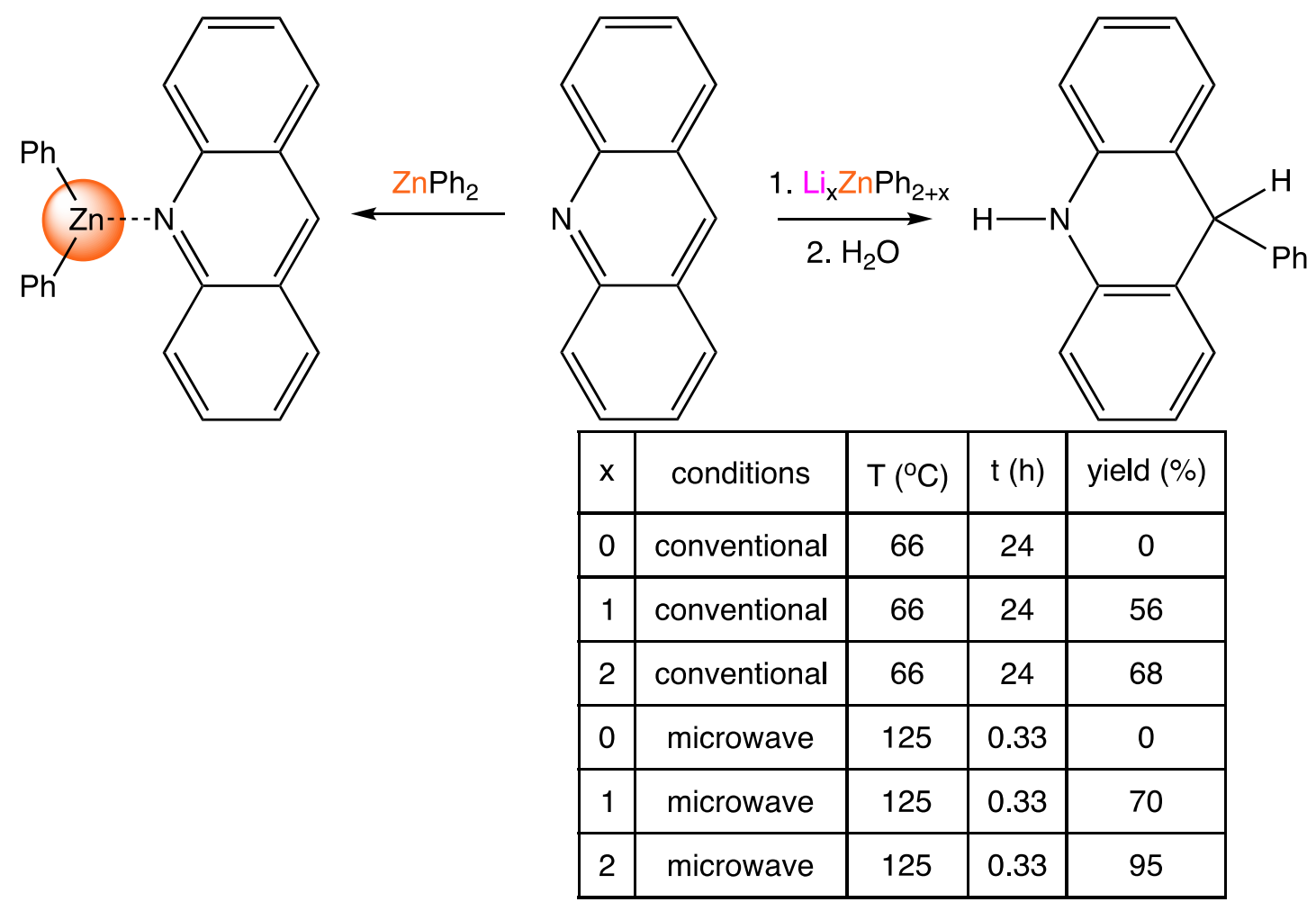

External oxidation by one and a half molar equivalents of DDQ (2,3-dichloro-5,6dicyano-1,4-benzoquinone) restored the aromaticity of the substituted acridine products in 71 and $61 \%$ respectively.

The alkali metal amidozincates described to date have all been derived from monoamines. Broadening the class to diamines, the bidentate aminopyrrole $\left[\mathrm{C}_{4} \mathrm{H}_{3} \mathrm{NH}\left(2-\mathrm{CH}_{2} \mathrm{NH}^{t} \mathrm{Bu}\right)\right]$ was converted to alkali metal derivatives through reaction with $n$-butyllithium, sodium hydride or potassium hydride then treated with diethylzinc to undergo cocomplexation reactions that were heated to $100{ }^{\circ} \mathrm{C}$ in toluene. At this temperature the aminopyrrole ligands are deprotonated a second time and ligand dianions form in a series of alkali metal alkylzincates of formulae $\left[\mathrm{Li}(\mathrm{TMEDA})_{2}\right]\left[\mathrm{Li}\left\{\mathrm{C}_{4} \mathrm{H}_{3} \mathrm{~N}\left(2-\mathrm{CH}_{2} \mathrm{~N}\left({ }^{\mathrm{t} B u}\right) \mathrm{ZnEt}\right)\right\}_{2}\right] \quad$ (159), $\quad$ [[Na(TMEDA) $\mathrm{Na}\left\{\mathrm{C}_{4} \mathrm{H}_{3} \mathrm{~N}(2-\right.$ $\left.\left.\left.\mathrm{CH}_{2} \mathrm{~N}\left({ }^{\mathrm{t}} \mathrm{Bu}\right) \mathrm{ZnEt}\right)\right\}_{2}\right] \quad(\mathbf{1 6 0}), \quad\left[\mathrm{Na}(\mathrm{THF})_{2} \mathrm{Na}\left\{\mathrm{C}_{4} \mathrm{H}_{3} \mathrm{~N}\left(2-\mathrm{CH}_{2} \mathrm{~N}\left({ }^{\mathrm{B}} \mathrm{Bu}\right) \mathrm{ZnEt}\right)\right\}_{2}\right] \quad$ (161) and $\left[\mathrm{K}(\mathrm{THF}) \mathrm{K}\left\{\mathrm{C}_{4} \mathrm{H}_{3} \mathrm{~N}\left(2-\mathrm{CH}_{2} \mathrm{~N}\left({ }^{t} \mathrm{Bu}\right) \mathrm{ZnEt}\right)\right\}_{2}\right](162) .{ }^{203}$ While the sodium alkyl zincates and potassium alkyl zincates form polymeric chain structures with a linear or zig-zag 
arrangement, the lithium congener 159 prefers a charged-separated complex where the lithium center in the anionic moiety occupies the core of a sandwich between two $\eta^{5}$ pyrrolyl rings (Figure 29).

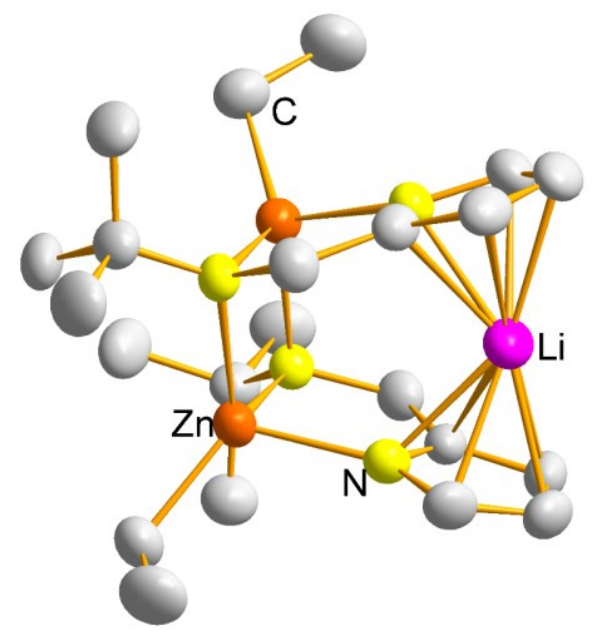

Figure 29. Molecular structure of the anionic moiety of $\left[\operatorname{Li}(T M E D A)_{2}\right]\left[\mathrm{Li}_{\{}\left\{\mathrm{C}_{4} \mathrm{H}_{3} \mathrm{~N}(2-\right.\right.$ $\left.\left.\left.\mathrm{CH}_{2} \mathrm{~N}\left({ }^{\mathrm{t}} \mathrm{Bu}\right) \mathrm{ZnEt}\right)\right\}_{2}\right]$ (159).

Note that the reactivity of these dianion-ligated zincates has not been investigated. Another anionic zincate has been prepared in $\left[2,6-(\mathrm{ArN}=\mathrm{CH})_{2} \mathrm{C}_{6} \mathrm{H}_{3} \mathrm{ZnCl}_{2} \mathrm{Li}(\mathrm{THF})_{3}\right](\mathbf{1 6 3})$, which contains a bis(imino)phenyl NCN pincer ligand. ${ }^{204}$ However, forming a contacted ion pair in which the $\left[\mathrm{Li}(\mathrm{THF})_{3}\right]^{+}$cation binds directly to the anionic moiety through one of the chloride atoms, this zincate reacts with LiHBEt $_{3}$ to initiate a hydride transfer to the $\mathrm{C}=\mathrm{N}$ imine group to produce the unusual dimeric zinc complex $\left[\eta^{1}(\mathrm{C}), \mathrm{K}^{1}\left(\mathrm{~N}^{\text {imine }}\right)-2-(\mathrm{ArN}=\mathrm{CH})-6-\left(\mathrm{Zn}-\mathrm{N}(\mathrm{Ar}) \mathrm{CH}_{2}\right) \mathrm{C}_{6} \mathrm{H}_{3} \mathrm{Zn}\right]_{2}$ (164, Scheme 111).

Scheme 111. Preparation of the novel dizinc diimine derivative $\left[\eta^{1}(C), K^{1}\left(N^{\text {imine }}\right)-2-\right.$ ( $\left.\mathrm{ArN}=\mathrm{CH})-6-\left(\mathrm{Zn}-\mathrm{N}(\mathrm{Ar}) \mathrm{CH}_{2}\right) \mathrm{C}_{6} \mathrm{H}_{3} \mathrm{Zn}\right]_{2}$ (164) made via an intermediate zinc hydride species, starting from lithium zincate 163 (molecular structure shown in inset). 


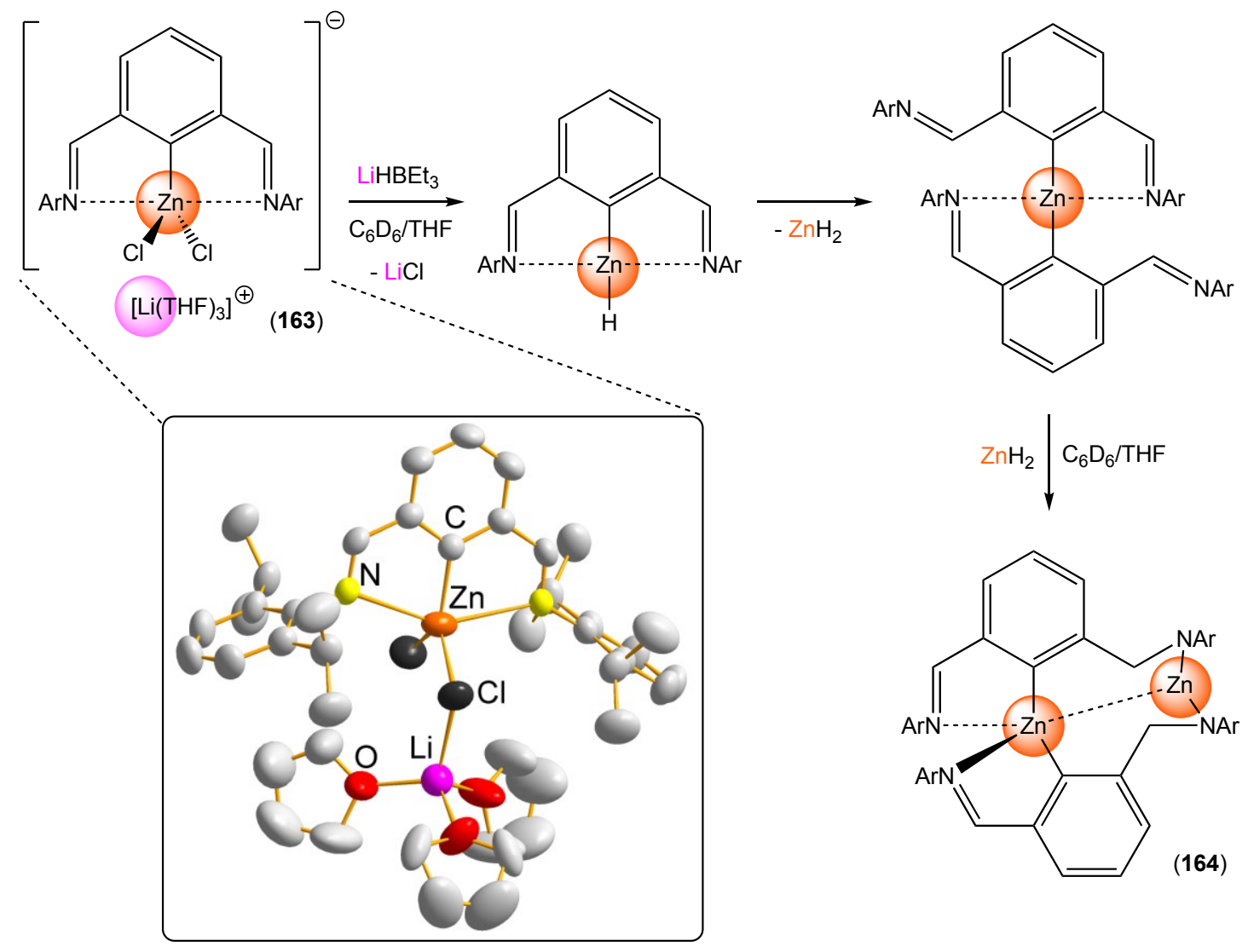

Deviations from the common Weiss motif can also be found when the multifunctional 2,2'-dipyridylamide [DPA, $\left(2-\mathrm{NC}_{5} \mathrm{H}_{4}\right)_{2} \mathrm{~N}$ ] ligand is used (Scheme 112). ${ }^{205}$

Scheme 112. Preparation of the "disodium zincate" [(TMEDA $\left.)_{2} \mathrm{Na}_{2}(\mu-\mathrm{DPA})_{2} \mathrm{Zn}\left({ }^{\mathrm{t} B u}\right)_{2}\right]$ (165).

$\underset{\mathrm{Me}_{2}}{\stackrel{\mathrm{Ne}}{\mathrm{Me}_{2}}} \stackrel{\mathrm{Na}}{\mathrm{HN}(2-p y)_{2}}$

(123)

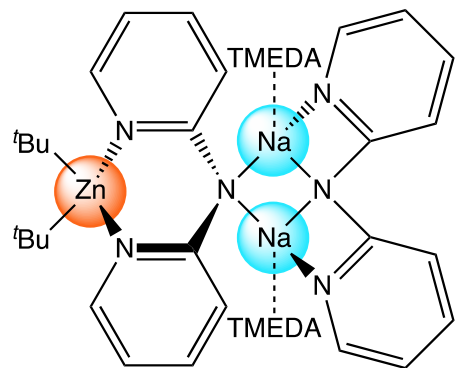

(165) 
Anticipating a simple transamination reaction, [(TMEDA) Na(TMP) $\left.\left({ }^{t} \mathrm{Bu}\right) \mathrm{Zn}\left({ }^{t} \mathrm{Bu}\right)\right](\mathbf{1 2 3})$ reacts with DPA to form the "disodium zincate" [(TMEDA $\left.)_{2} \mathrm{Na}_{2}(\mu-\mathrm{DPA})_{2} \mathrm{Zn}\left({ }^{\mathrm{t}} \mathrm{Bu}\right)_{2}\right](\mathbf{1 6 5})$, the structure of which has been described as a cocomplex between a dimeric TMEDAchelated sodium amide and a bis(alkyl) zinc monomer connected through two dative $\mathrm{Zn}-\mathrm{N}$ bonds involving pyridine $\mathrm{N}$ atoms. More structural diversity is apparent when $\mathrm{NaDPA}$ or the potassium congener KDPA is treated with ${ }^{t} \mathrm{Bu}_{2} \mathrm{Zn}$ in the presence of THF. Now, instead of a contact ion pair, the charge-separated, mono-alkali-metal dizinc complexes $\left[\left\{\mathrm{M}(\mathrm{THF})_{6}\right\}^{+}\left\{\mathrm{Zn}\left({ }^{t} \mathrm{Bu}\right)_{2}(\mathrm{DPA}) \mathrm{Zn}\left({ }^{t} \mathrm{Bu}\right)_{2}\right\}^{-}\right]((\mathrm{M}=\mathrm{Na}, 166$; or $\mathrm{K}, 167)$ are formed. Synergistic reactivity in these DPA zinc systems was demonstrated in benchmark reactions with benzophenone, table 6 . While homonuclear ${ }^{t} \mathrm{Bu}_{2} \mathrm{Zn}$ is essentially inert to benzophenone, the three-zincate species produce competitive yields of the challenging para-addition product 4-tert-butylbenzophenone. Intriguingly, when a 1:1 mixture of ${ }^{t} \mathrm{Bu}_{2} \mathrm{Zn}$ and benzophenone was treated with only 10 mol\% of the sodium amide $\left[\{(\mathrm{TMEDA}) \mathrm{Na}(\mathrm{DPA})\}_{2}\right]$, presumably generating $10 \mathrm{~mol} \%$ of $\mathbf{1 6 5}$ in situ in hexane solution, the yield of 4-tert-butylbenzophenone increased from $11 \%$ at room temperature to $52 \%$ under reflux conditions, consistent with a degree of recycling of the sodium amide.

Table 6. Reactivity of various neutral and anionic zinc reagents with benzophenone. 


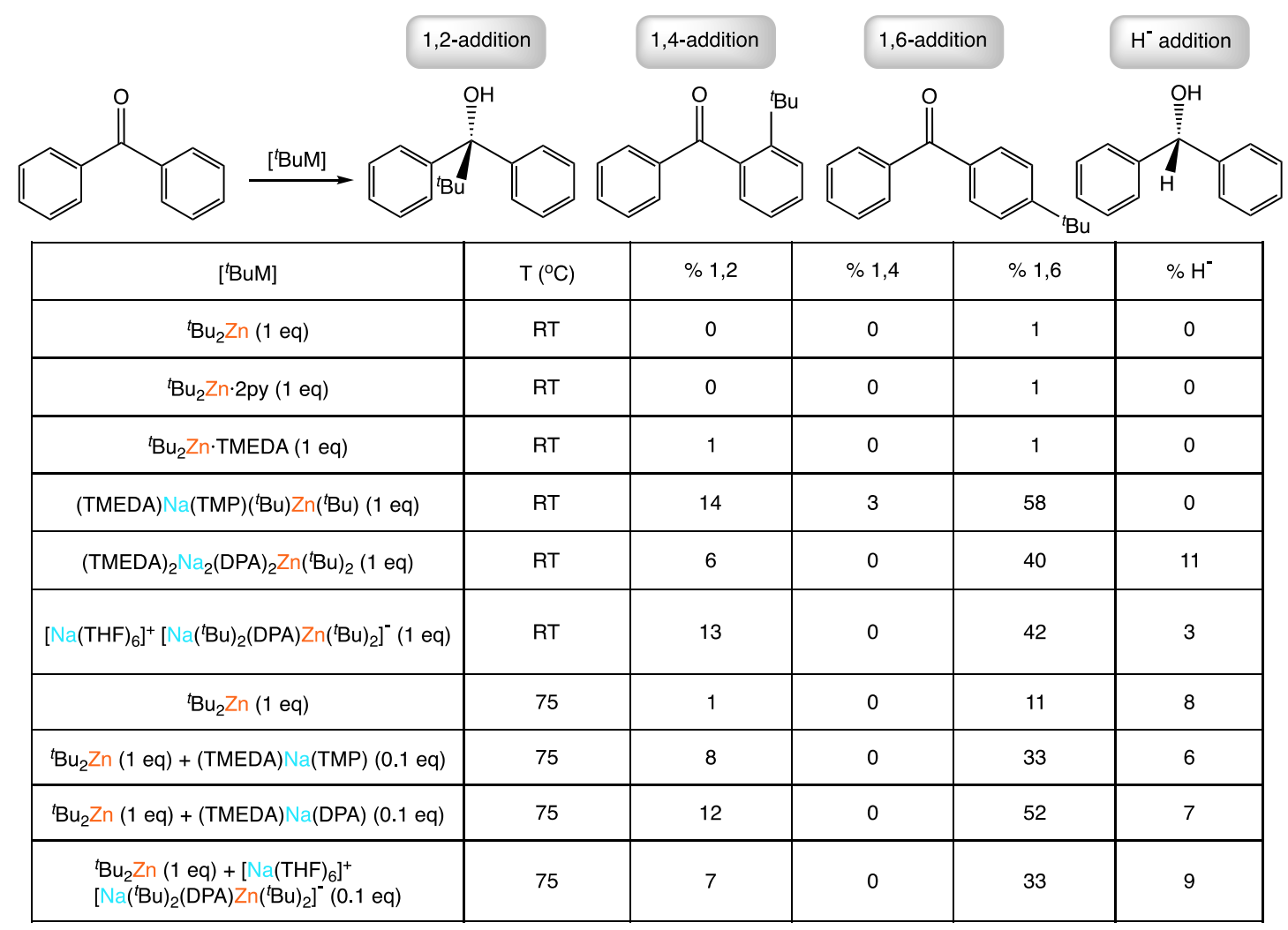

Cocomplexation has proved an efficient method for preparing other novel zincate formulations. This includes a homologous series of donor-solvent-free alkali-metal tris(trimethylsilylmethyl)zincates $\left[\left\{\mathrm{MZn}\left(\mathrm{CH}_{2} \mathrm{SiMe}_{3}\right)_{3}\right\}_{\infty}\right](\mathrm{M}=\mathrm{Li}, 168 ; \mathrm{Na}, 169 ; \mathrm{K}, 170$; Scheme 113). ${ }^{206}$ Made in and crystallized from hexane solution, these zincates exhibit unusual supramolecular assemblies possessing a three-fold combination of $\mathrm{M}-\mathrm{CH}_{2}$, $\mathrm{Zn}-\mathrm{CH}_{2}$ and $\mathrm{M} \cdot \mathrm{Me}$ contacts, with 168 exhibiting a linear chain structure; whereas the sodium and potassium congeners 169 and 170 display more complicated 3D and 2D coordination networks respectively. These supramolecular structures are easily deaggregated by donor solvents to monomers as in [(PMDETA)LiZn $\left.\left(\mathrm{CH}_{2} \mathrm{SiMe}_{3}\right)_{3}\right](\mathbf{1 7 1})$ and $\left[(\mathrm{TMEDA})_{2} \mathrm{NaZn}\left(\mathrm{CH}_{2} \mathrm{SiMe}_{3}\right)_{3}\right]$ (172). 
Scheme 113. Formation of molecular $\left[(\mathrm{PMDETA}) \mathrm{LiZn}\left(\mathrm{CH}_{2} \mathrm{SiMe}_{3}\right)_{3}\right]$ (171) and [(TMEDA $\left.)_{2} \mathrm{NaZn}\left(\mathrm{CH}_{2} \mathrm{SiMe}_{3}\right)_{3}\right]$ (172) by cocomplexation and Lewis base donor addition, and polymeric $\left[\left\{\mathrm{KZn}\left(\mathrm{CH}_{2} \mathrm{SiMe}_{3}\right)_{3}\right\}_{\infty}\right](\mathbf{1 7 0})$ in the absence of a donor solvent.

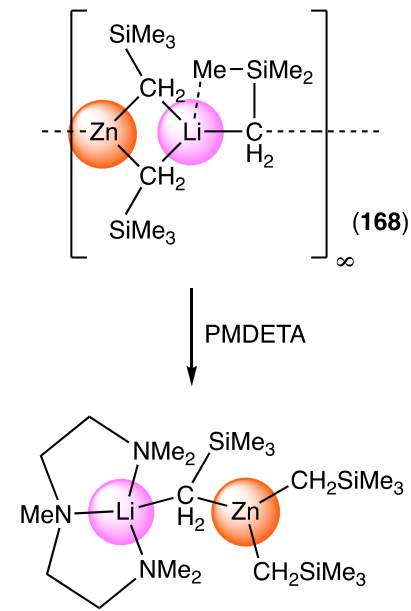

(171)
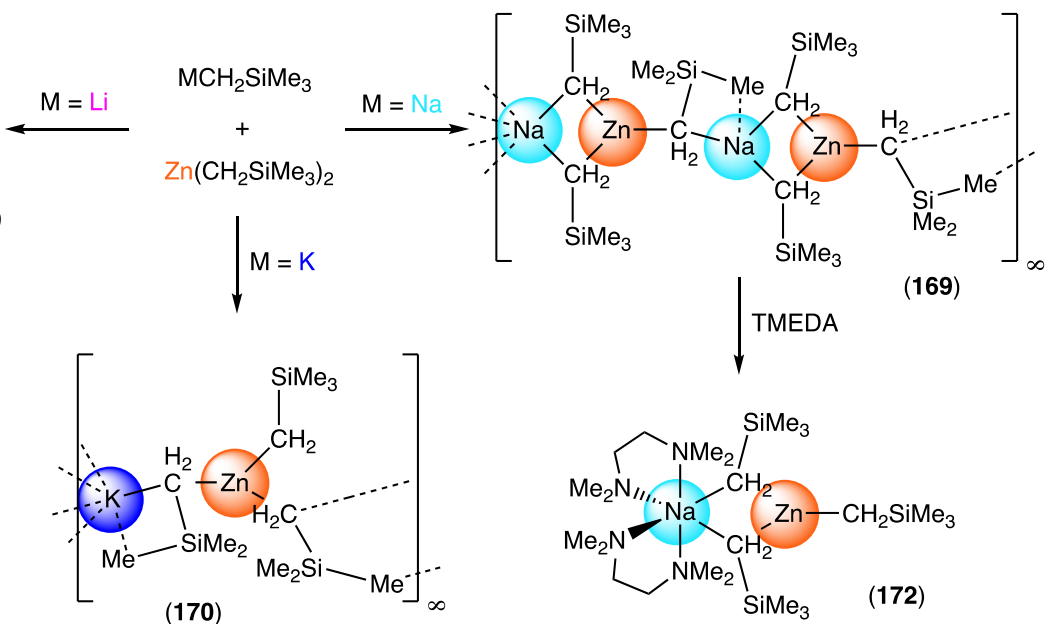

From an amide sterically less hindered than TMP to one that is slightly more sterically hindered, the related piperidine amide, dispiro[cyclohexane-2,2'-piperidine-6',2"cyclohexane [CPC-(H)], has also been initiated into bimetallic chemistry. The lithium amide derivative LiCPC forms a common trimeric structure with a six-membered ( $\mathrm{LiN})_{3}$ ring. It reacts with diethylzinc in the presence of the donor solvents THF or TMEDA to form zincate complexes (173 and $\mathbf{1 7 4}$, Scheme 114). A reactivity study on the iodination of tert-butyl 3-bromobenzoate with $\mathrm{Li}(\mathrm{CPC}) \mathrm{ZnEt}_{2}$ showed a slightly improved regioselectivity for the 6-position compared to that with the analogous Li(TMP)ZnEt ${ }_{2}$ complex, which suggested a moderately larger steric bulk of CPC in comparison to that of the TMP ligand complex. ${ }^{207}$ 
Scheme 114. Preparation of lithium diethylzincate complexes 173 and 174 based on the deprotonated version of the dispiro[cyclohexane-2,2'-piperidine-6', $2^{\prime \prime}$ cyclohexane $[\mathrm{CPC}-(\mathrm{H})]$ ligand.
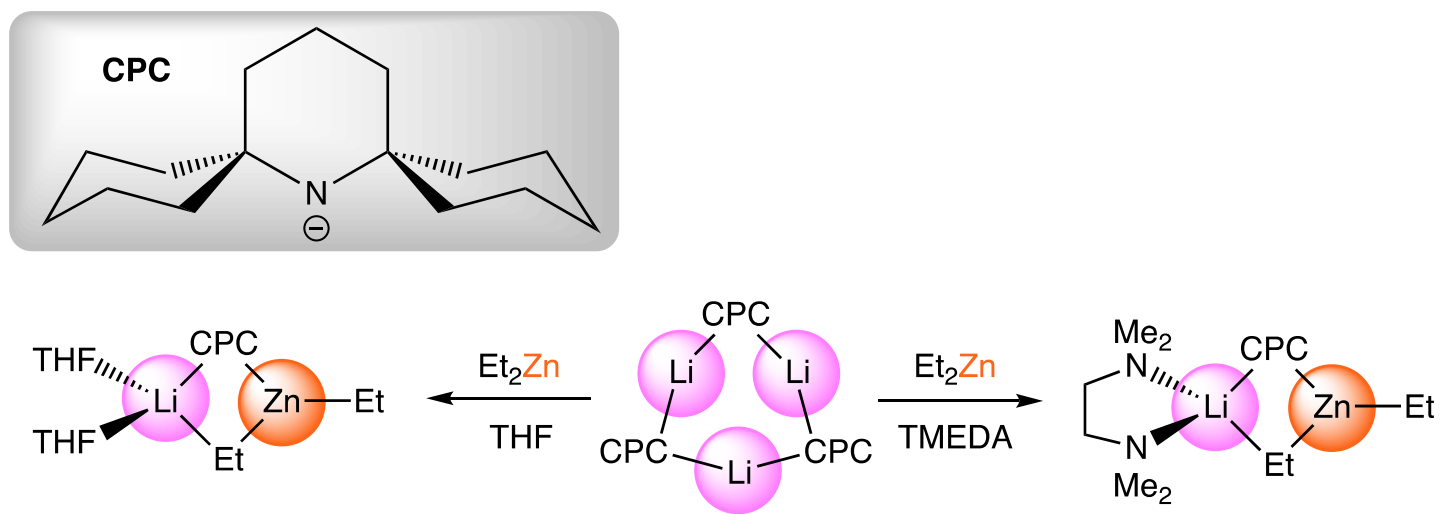

(173)

(174)

Cocomplexation was also used to prepare the monomeric potassium zincate $\left[{ }^{t} \mathrm{Bu}_{2} \mathrm{Zn}\left(\mathrm{PhCH}_{2}\right) \mathrm{K} \cdot \mathrm{Me}_{6} \mathrm{TREN}\right]$ (175) by treating Me${ }_{6} \mathrm{TREN}$ [tris( $N, N$-dimethyl-2aminoethyl)amine, $\mathrm{N}\left(\mathrm{CH}_{2} \mathrm{CH}_{2} \mathrm{NMe}_{2}\right)_{3}$ ] stabilized benzyl potassium with the dialkylzinc compound (Scheme 115). ${ }^{208}$

Scheme 115. A cocomplexation reaction producing the monomeric potassium zincate complex $\left[{ }^{t} \mathrm{Bu}_{2} \mathrm{Zn}\left(\mathrm{PhCH}_{2}\right) \mathrm{K} \cdot \mathrm{Me}_{6} \mathrm{TREN}\right](\mathbf{1 7 5})$.

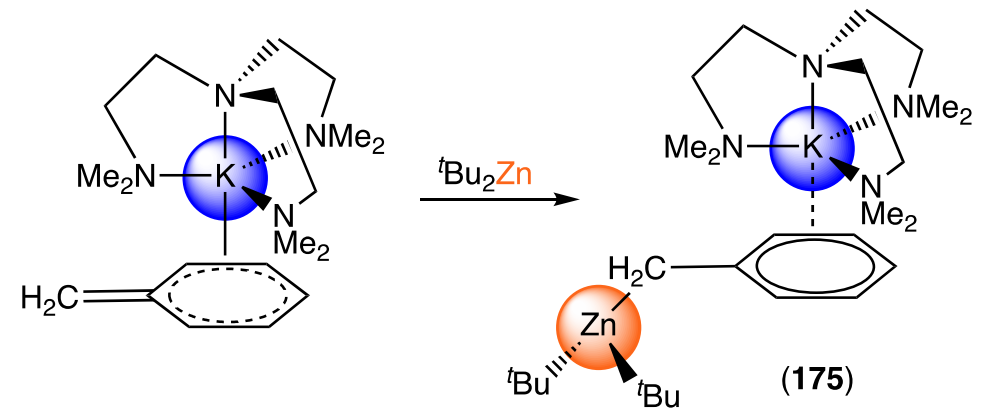


A novel feature of this structure is that the benzyl ligand functions as a ditopic $\sigma / \pi$ bridging ligand between the two metals. In the previously reported homometallic monomeric series $\mathrm{PhCH}_{2} \mathrm{M} \cdot \mathrm{Me}_{6} \operatorname{TREN}(\mathrm{M}=\mathrm{Li}, \mathrm{Na}, \mathrm{K})$ the $\mathrm{M}$-anion bonding interaction switches from predominately localized $\sigma\left(\mathrm{sp}^{3} \mathrm{CH}_{2}\right)$ to mainly delocalized $\pi\left(\mathrm{sp}^{2} \mathrm{CH}_{2}\right)$ on increasing cation size from Li to K. ${ }^{209}$ However, in heterometallic $\mathbf{1 7 5}$ the small zinc atom relocalizes the negative charge back on to the lateral $\mathrm{CH}_{2} \mathrm{arm}$, an interpretation supported by NMR chemical shifts, to give a complex best described as a contacted ion pair potassium zincate, where the softer $K$ center $\pi$ bonds to the arene face.

Cocomplexation reactions are not always so straightforward. For example, adding one equivalent of the donor solvent TMEDA to a 1:1 hexane mixture of $\mathrm{Zn}\left(\mathrm{CH}_{2} \mathrm{SiMe}_{3}\right)_{2}$ and phenyllithium at ambient temperature resulted in formation of the homoleptic alkylzincate [(TMEDA) LiZn $\left.\left(\mathrm{CH}_{2} \mathrm{SiMe}_{3}\right)_{3}\right]$ (176, Figure 30) instead of the expected heteroleptic complex $\left[(\mathrm{TMEDA}) \operatorname{LiZn}(\mathrm{Ph})\left(\mathrm{CH}_{2} \mathrm{SiMe}_{3}\right)_{2}\right] .{ }^{210}$ The formation of the homoleptic alkylzincate is presumed to be due to a disproportionation process.

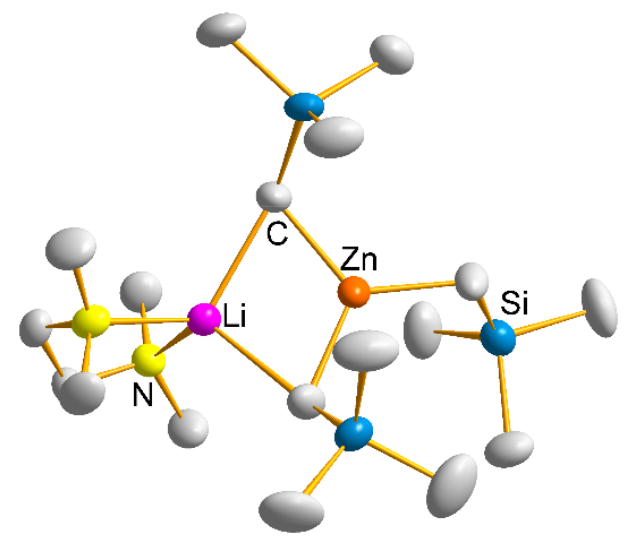

Figure 30. Molecular structure of $\left[(T M E D A) L i Z n\left(\mathrm{CH}_{2} \mathrm{SiMe}_{3}\right)_{3}\right]$ (176). Note the previously mentioned PMDETA variant 171. 
First synthesized in $2002,{ }^{211}$ the potassium heteroleptic zincate complex $\left[\mathrm{KZn}(\mathrm{HMDS})_{2}\left(\mathrm{CH}_{2} \mathrm{Ph}\right)\right]_{\infty}$ (177) remade in 2018 by an alternative cocomplexation approach between potassium benzyl and zinc amide (Scheme 116), acts as a catalyst for preparing diarylalkanes and displays good activity and chemoselectivity. ${ }^{212}$

Scheme 116. Cocomplexation synthesis of the potassium heteroleptic zincate complex $\left[\mathrm{KZn}(\mathrm{HMDS})_{2}\left(\mathrm{CH}_{2} \mathrm{Ph}\right)\right]_{\infty}(\mathbf{1 7 7})$, a precatalyst for the addition of diphenylmethane to styrenes and conjugated dienes.

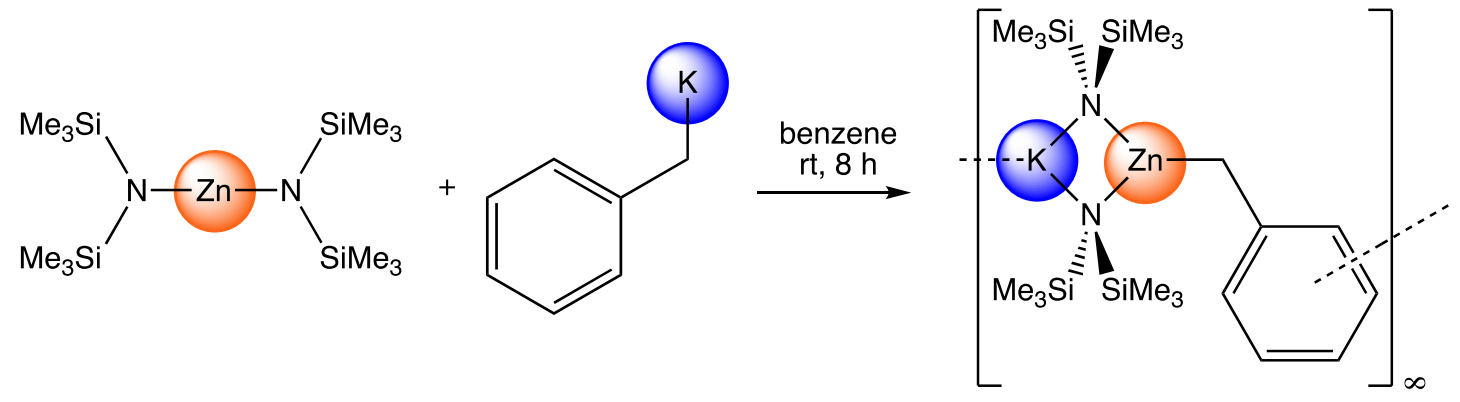

(177)

Demonstrating its synergistic efficiency in optimization experiments, 10 mol\% of 177 catalyses the addition of diphenylmethane to styrene in benzene at $70^{\circ} \mathrm{C}$ in a $90 \%$ yield (Scheme 117); whereas its monometallic components $\mathrm{KHMDS}$ and $\mathrm{Zn}\left(\mathrm{CH}_{2} \mathrm{Ph}\right)_{2}$ produce only $11 \%$ and $<5 \%$ respectively. Scope was elaborated for various styrenes and conjugated dienes (16 examples, yields $11 \%$ to $98 \%$ ) and for various diarylmethanes (16 examples, yields $10 \%$ to $96 \%$ ). The poor yields came with 4 halostyrenes and 4-haloarylmethanes. A plausible reaction pathway was presented (Scheme 117).

Scheme 117. Postulated catalytic cycle for the zincate-catalyzed addition of diphenylmethane to styrenes and conjugated dienes. 


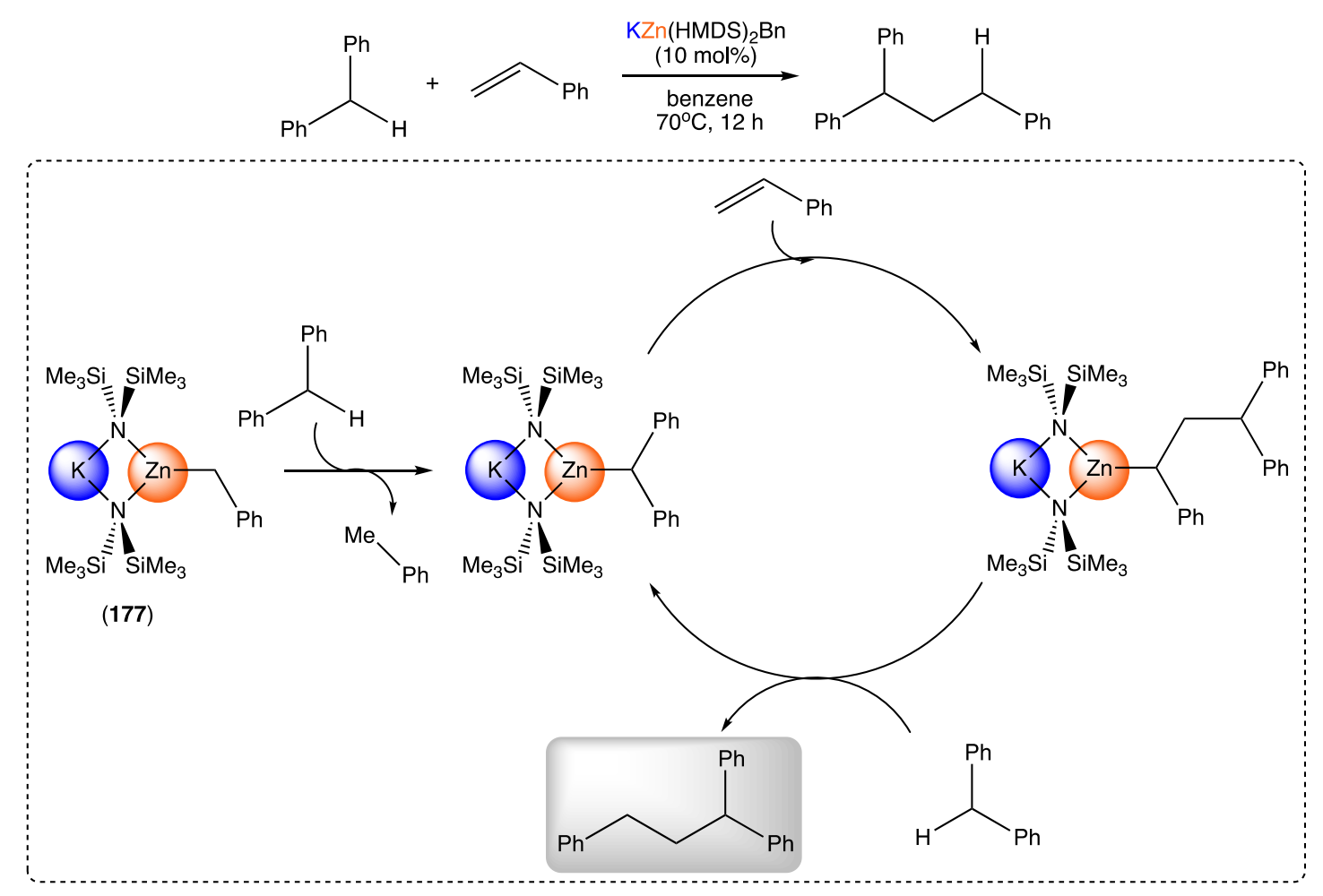

\subsubsection{Trans-Metal-Trapping}

Salt metathesis reactions are commonly employed to transfer ligands on reactive alkali metals to less reactive, softer metals. In reactions related to the aforementioned trans-metal-trapping processes where the exchange does not go to completion but instead stops at an intermediate crossover point, the contacted ion heteroleptic zincate complexes $\left[(\mathrm{THF})_{3} \mathrm{Li}(\mu-\mathrm{Cl}) \mathrm{Cl} \mathrm{Zn}\left(\mu\right.\right.$-dipp-dabqdi) $\left.\mathrm{ZnCl}(\mu-\mathrm{Cl}) \mathrm{Li}(\mathrm{THF})_{3}\right] \quad$ (178), $\left[(\mathrm{DME})_{2} \mathrm{Li}(\mu-\mathrm{Cl}) \mathrm{ClZn}\left(\mu-\right.\right.$ dippdabqdi) $\left.\mathrm{ZnCl}(\mu-\mathrm{Cl}) \mathrm{Li}(\mathrm{DME})_{2}\right] \quad(\mathbf{1 7 9}), \quad$ and $\quad\left[(\mathrm{DME})_{2} \mathrm{Na}(\mu-\right.$ $\mathrm{Cl})_{2} \mathrm{Zn}\left(\mu\right.$-dipp-dabqdi) $\left.\mathrm{Zn}(\mu-\mathrm{Cl})_{2} \mathrm{Na}(\mathrm{DME})_{2}\right]$ (180), have been synthesized by reacting the corresponding alkali metal dipp-dabqdi compounds with zinc chloride (dippdabqdiH $_{2}=$ 2,5-diamino-1,4-benzoquinonediimine; DME = 1,2-dimethoxyethane)..$^{213}$ Formally, the zinc chloride has inserted into the alkali metal nitrogen bonds to 
generate Weiss-type arrangements, though in the lithium structures the Li centers only coordinate to one chloride atom (Scheme 118).

Scheme 118. Preparation of zincate complexes 178-180 derived from dipp-dabqdiH $\mathrm{H}_{2}$ (= 2,5-diamino-1,4-benzoquinonediimine) where salt metathesis does not take place.

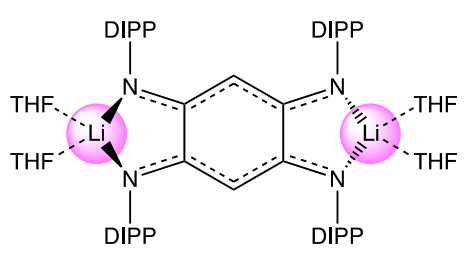

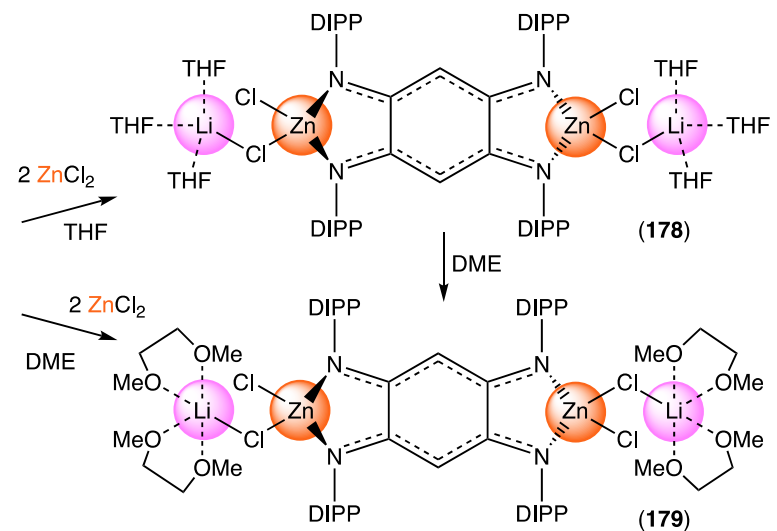

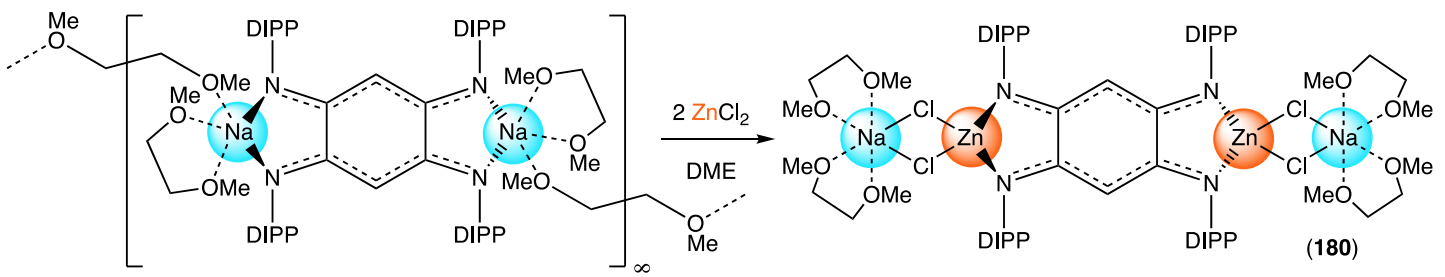

In a similar vein, a fluorinated $\beta$-aminoimine compound has been lithiated by $n$ butyllithium then reacted with zinc iodide or zinc bromide to generate the $\beta$ diketiminate zinc complexes $\left[\mathrm{HC}(\mathrm{CMeNAr})_{2}\right] \mathrm{Zn}(\mathrm{I})_{2} \mathrm{Li}_{(}\left(\mathrm{Et}_{2} \mathrm{O}\right)_{2} \quad$ (181) and $\left[\mathrm{HC}(\mathrm{CMeNAr})_{2}\right] \mathrm{Zn}(\mathrm{Br})_{2} \mathrm{Li}\left(\mathrm{Et}_{2} \mathrm{O}\right)_{2}\left(\mathbf{1 8 2}, \mathrm{Ar}=2,6-\mathrm{F}_{2} \mathrm{C}_{6} \mathrm{H}_{3}\right) .{ }^{214}$ Akin to part of a trans-metaltrapping procedure, the contacted ion pair structure of the halide complexes (Scheme 119) show that the $\mathrm{ZnX} X_{2}$ units insert into the $\mathrm{Li}-\mathrm{N}$ bonds of the lithium precursor giving the $\mathrm{Zn}$ atom a tetrahedral $\mathrm{N}_{2} \mathrm{X}_{2}$ coordination, whereas the Li center binds to the two halide ions and two ether solvent molecules. 
Scheme 119. Preparation of a mixed lithium - zinc complex (182) featuring a fluorinated $\beta$-aminoimine and bromide ligands, where insertion akin to that in transmetal-trapping occurs as opposed to a metathesis.

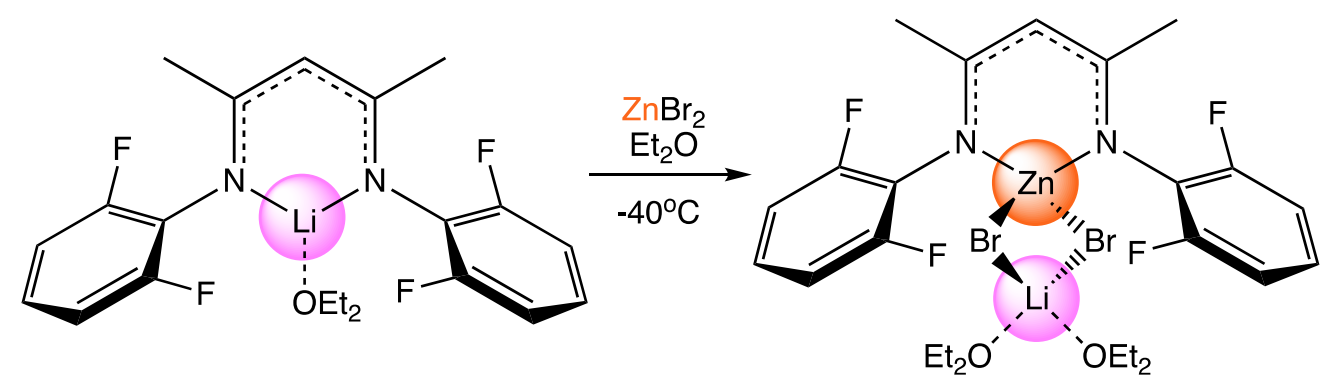

(182)

While the first cyclobutadiene complex that is substituted by four 2-pyridyl units, namely $\left[K([18] c r o w n-6)\left\{C p * F e\left(\eta 4-C_{4} p_{4}\right)\right\}\right] \quad(183)$ was not produced through a synergistic process, it displays a novel bonding feature in which the potassium center coordinates to one pyridyl group. ${ }^{215}$ When in turn this complex is reacted with zinc chloride, the dizinc adduct $[\mathrm{K}([18] \mathrm{crown}-6)(\mathrm{THF})]\left[\mathrm{Cp} * \mathrm{Fe}\left(\eta^{4}-\mathrm{C}_{4} \mathrm{py}_{4}\right)\left(\mathrm{ZnCl}_{2}\right)_{2}\right](\mathbf{1 8 4})$ is formed, where pairs of pyridyl $\mathrm{N}$ atoms act as chelates to the tetrahedral zinc centers (Figure 31).

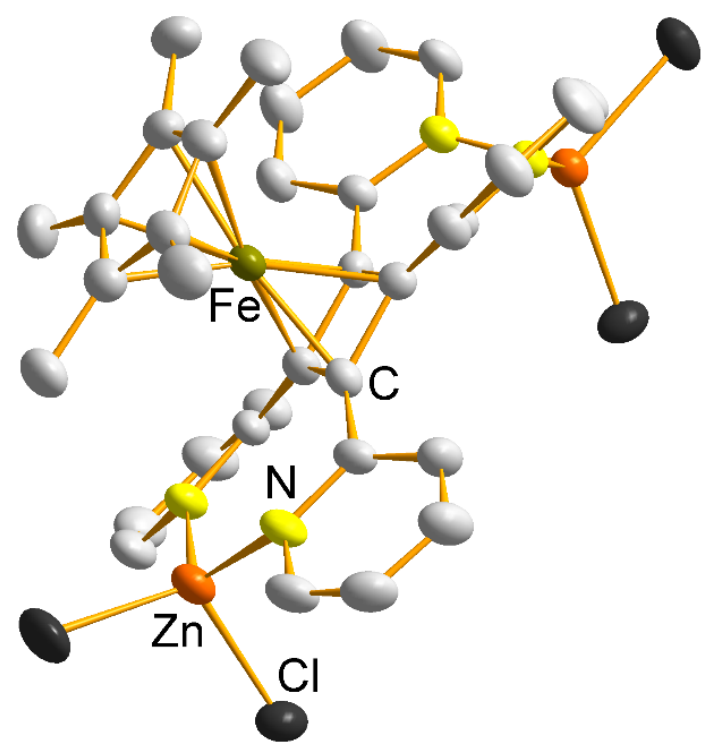


Figure 31. Molecular structure of the anionic moiety of [K([18]crown6)(THF)][Cp* Fe $\left.\left(\eta^{4}-\mathrm{C}_{4} \mathrm{py} \mathrm{P}_{4}\right)\left(\mathrm{ZnCl}_{2}\right)_{2}\right](\mathbf{1 8 4})$.

A special category of dilithium compound that exhibits cooperative effects due to the close proximity of their Li centers, 1,4-dilithio-1,3-dienes (see also section 2.2.3) can be employed in reactions with diaryl diazomethanes in 1:2 stoichiometries to prepare 1-imino-pyrrole and indole derivatives (Scheme 120). ${ }^{216}$ Interestingly, the intermediate in this reaction, when treated with zinc chloride did not undergo full transmetalation, but instead only one equivalent of lithium chloride was eliminated leaving a monolithium dizinc complex (185) that shows a rare aza-allyl $\left(\mu_{2}-\eta^{1}: \eta^{1}\right)$ coordination mode.

Scheme 120. An incomplete metathesis reaction leading to a monolithium dizinc complex 185 containing a mixture of indole and chloride ligands.

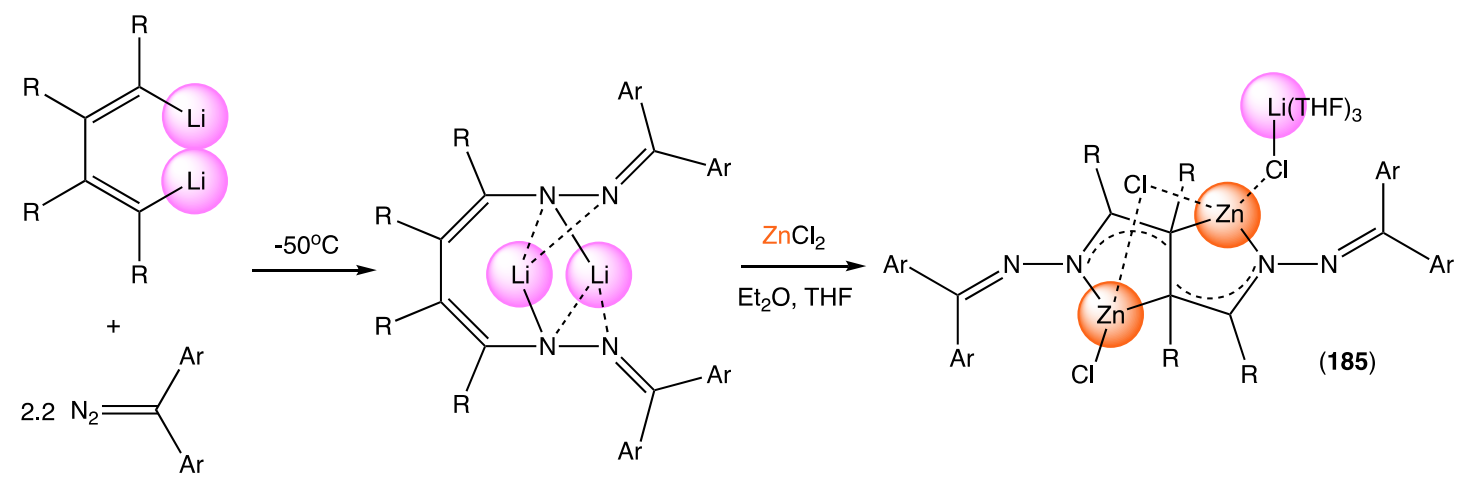

\subsubsection{Miscellaneous Examples}

When the tripodal organotrisilanol, ${ }^{t} \mathrm{BuSi}\left(\mathrm{OSiMe}{ }_{2} \mathrm{OH}\right)_{3}$, is triply deprotonated by three equivalents of $\mathrm{MeONa}$, it can be transformed into a zincate by adding zinc triflate, $\mathrm{Zn}(\mathrm{OTf})_{2}$. Interestingly while this generates a typical Weiss motif in $\left[\left\{\left(\mathrm{Et}_{2} \mathrm{O}\right)_{2} \mathrm{Na}\left[{ }^{t} \mathrm{BuSi}\left(\mathrm{OSiMe}_{2} \mathrm{O}\right)_{3}\right] \mathrm{Zn}\right\}_{2}\right](\mathbf{1 8 6})$ with tetrahedral zinc centers; replacing zinc 
by iron via $\mathrm{Fe}(\mathrm{OTf})_{2}$, eliminates one " $\mathrm{Me}_{2} \mathrm{SiO}$ " unit to generate $\left[\left\{\left(\mathrm{Et}_{2} \mathrm{O}\right)_{2} \mathrm{Na}\right.\right.$ $\left.\left.{ }^{t} \mathrm{BuSi}\left(\mathrm{OMe}_{2} \mathrm{SiO}\right)_{2}(\mathrm{O}) \mathrm{Fe}\right\}_{2}\right]$ (187) containing square planar Fe centers within high-spin $\mathrm{FeO}_{4}$ units (Scheme 121). ${ }^{217}$ Switching to the all-aryl silanol $\mathrm{PhSi}\left(\mathrm{OSiPh}{ }_{2} \mathrm{OH}\right)_{3}$ in a reaction with ${ }^{n} \mathrm{BuLi}$ and $\mathrm{ZnBr}_{2}$ in a 3:1 stoichiometry afforded $\left[\left\{\left(\mathrm{Et}_{2} \mathrm{O}\right)_{2} \mathrm{Li}\left[\mathrm{PhSi}\left(\mathrm{OSiPh}_{2} \mathrm{O}\right)_{3}\right] \mathrm{Zn}\right\}_{2}\right] \quad(\mathbf{1 8 8})$, in a closely related structure to the aforementioned sodium congener. Again, in the absence of the transition metal, the tripodal silanoxide ligand remains intact and there is no elimination of " $\mathrm{Me}_{2} \mathrm{SiO}$ " units. $^{218}$

Scheme 121. Contrasting chemistry of a sodium zincate (186) and a sodium ferrate (187) derived from the tripodal organotrisilanol, ${ }^{t} \mathrm{BuSi}\left(\mathrm{OSiMe}{ }_{2} \mathrm{OH}\right)_{3}$.

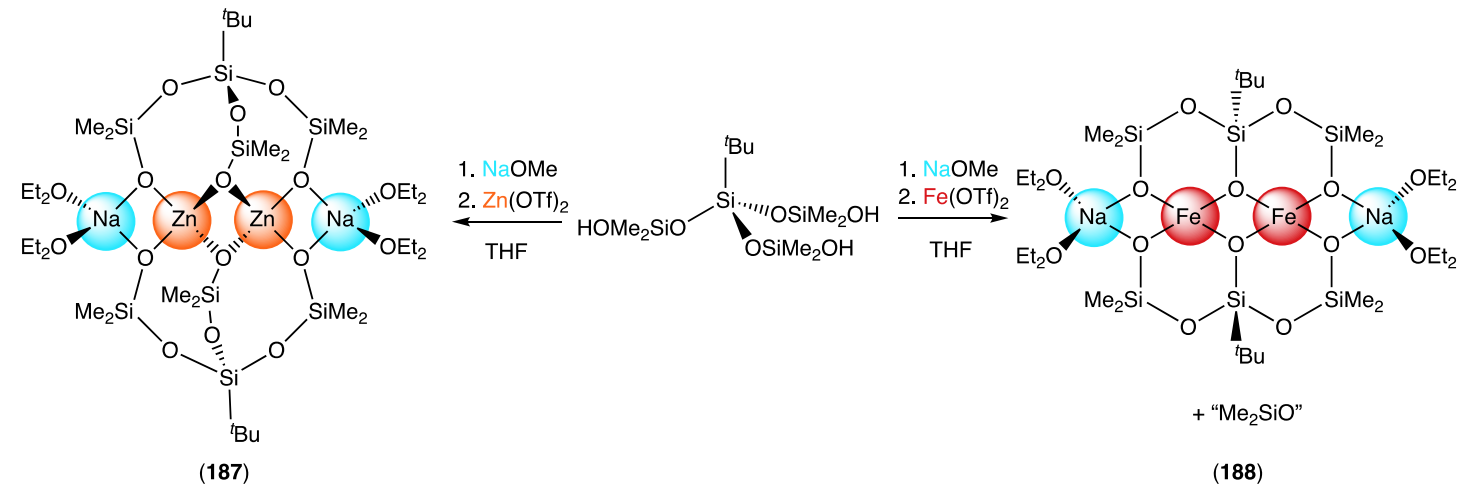

Another tripodal proligand, the sterically demanding trisphenol 2,6-bis(3,5-bis-t-butyl2-hydroxybenzyl)-4-t-butyl phenol has been converted into a series of THF-solvated heterobimetallic zincates and magnesiates (Scheme 122). ${ }^{219}$

Scheme 122. Preparation of dimeric tetranuclear lithium (189) and sodium zincates (190) containing a tripodal trisphenoxide ligand. 


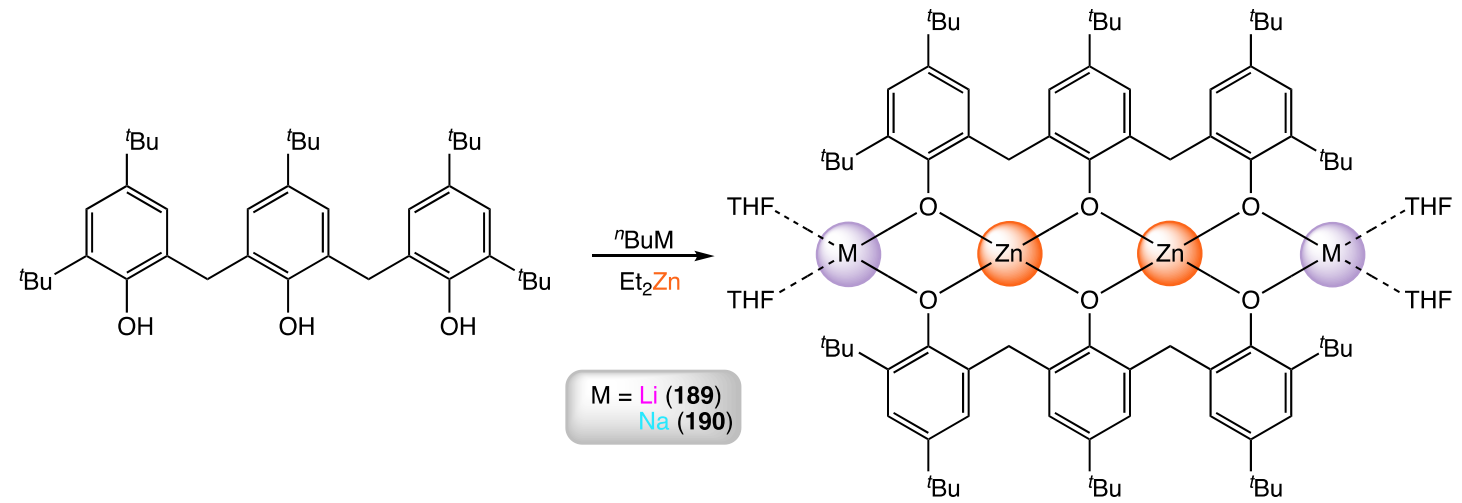

Sodium and lithium zincates have been prepared by treating the phenol with elemental sodium and $n$-butyllithium respectively then adding diethylzinc to complete the deprotonation of the hydroxide groups. Structurally the dimeric zincates and the analogous magnesiates adopt Weiss type structures with four tetrahedrally coordinated metal atoms in a collinear arrangement. Both 189 and 190 display excellent activities towards ring-opening polymerization of I-lactide and could also catalyze ROP of I-lactide with good conversions even under an air atmosphere. The lithium zincate outperformed its sodium congener in the coupling of carbon dioxide with cyclohexene oxide in the presence of ${ }^{n} \mathrm{Bu}_{4} \mathrm{NBr} /{ }^{n} \mathrm{Bu}{ }_{4} \mathrm{NCl}$ co-catalysts. A variation on TMP-zincate, the heteroleptic lithium vinylzincates [(TMEDA)Li(TMP) $\left.\left(\mathrm{CH}=\mathrm{CH}_{2}\right) \mathrm{Zn}\left(\mathrm{C}_{2} \mathrm{H}_{3}\right)\right]$ and [(PMDETA)K(TMP) $\left.\left(\mathrm{CH}=\mathrm{CH}_{2}\right) \mathrm{Zn}\left(\mathrm{CH}_{2} \mathrm{SiMe}_{3}\right)\right](\mathbf{1 9 2})^{178}$ have been described as potential model compounds for anionic ethene polymerization. ${ }^{220}$ The lithium vinyl-zincate 191 was synthesized from a mixture of vinyl lithium, $\mathrm{ZnTMP}_{2}$ and TMEDA in $n$-hexane solution in a stoichiometry (1:1:1) expected to yield monosubstituted [(TMEDA $\left.) \mathrm{Li}(\mathrm{TMP})\left(\mathrm{CH}=\mathrm{CH}_{2}\right) \mathrm{Zn}(\mathrm{TMP})\right]$. Instead the disubstituted vinyl product 191 was produced via a dismutation process. In a different approach, the potassium 
vinylzincate 192 was made by directly metalating ethene using the potassium-zincate complex [(PMDETA)K(TMP) $\left.\left(\mathrm{CH}_{2} \mathrm{SiMe}_{3}\right) \mathrm{Zn}\left(\mathrm{CH}_{2} \mathrm{SiMe}_{3}\right)\right]$ (193) in a synergistic metalation reaction (Scheme 123).

Scheme 123. Alternative preparations of $\left[(T M E D A) L i(T M P)\left(C H=\mathrm{CH}_{2}\right) \mathrm{Zn}\left(\mathrm{C}_{2} \mathrm{H}_{3}\right)\right]$ (191) via cocomplexation and $\left[(\mathrm{PMDETA}) \mathrm{K}(\mathrm{TMP})\left(\mathrm{CH}=\mathrm{CH}_{2}\right) \mathrm{Zn}\left(\mathrm{CH}_{2} \mathrm{SiMe}_{3}\right)\right]$ (192) via synergistic metalation, and their molecular structures.
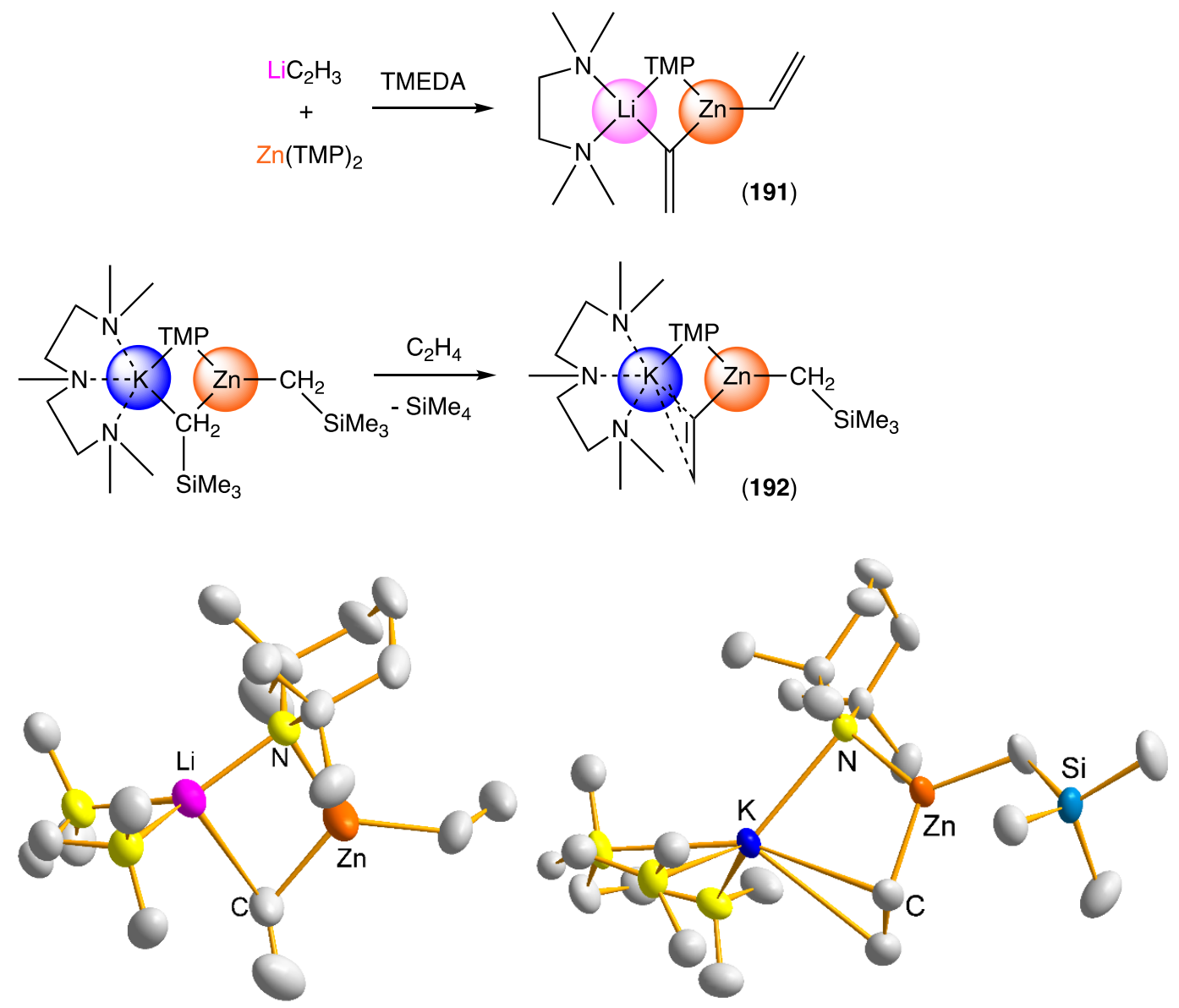

A series of DFT calculations on these model complexes revealed features similar to that found in DIPYE-BuLi (DIPYE = 1,2-dipyrrolidinoethane), which was earlier postulated as the transition state for the insertion reaction of ethene into the growing polymer chain initiated by tertiary-diamine/n-butyllithium complexes. ${ }^{221}$ 
Mixing ${ }^{n} \mathrm{BuLi}$ with either ${ }^{n, s} \mathrm{Bu}_{2} \mathrm{Mg}$ or $\mathrm{Me}_{2} \mathrm{Zn}$ in a 1:1 stoichiometric ratio with three equivalents of $h p p(H) \quad(1,3,4,6,7,8$-hexahydro-2H-pyrimido[1,2-a]pyrimidine) has produced homoleptic lithium-magnesium and lithium-zinc compounds of general formula $\mathrm{Li}_{2} \mathrm{M}_{2}(\mathrm{hpp})_{6}(\mathrm{M}=\mathrm{Mg}, 194 ; \mathrm{Zn}, 195)$. Synthesized with the prospect of realizing novel synergistic reactivity in metalation reactions with aromatic substrates as bases akin to those involving the TMP anion, these guanidinate hpp formulations proved inert in metalation reactions due to them forming stable cluster structures (Figure $32)^{222}$

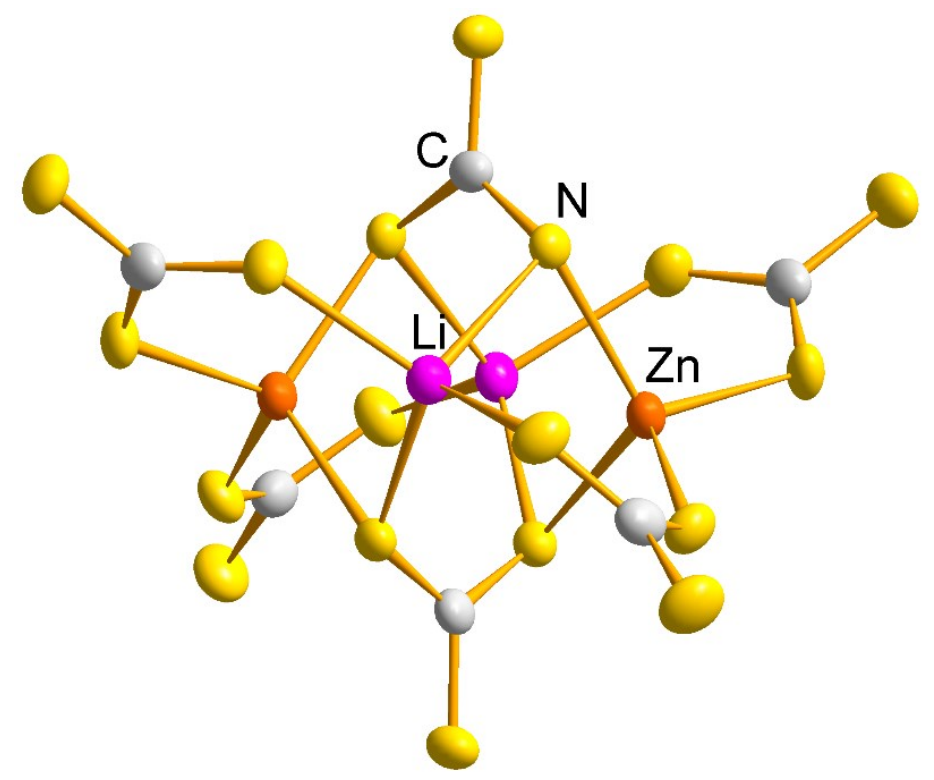

Figure 32. Molecular structure of $\operatorname{Li}_{2} Z n_{2}(h p p)_{6}(\mathbf{1 9 5})$, where $h p p(H)$ is $(1,3,4,6,7,8$ hexahydro-2H-pyrimido[1,2-a]pyrimidine). Note methylene groups have been omitted for clarity.

A strong synergistic effect is also in operation in the metal-mediated selective conversion of pyridine to a 1,4-dihydropyridine. ${ }^{223}$ While allylpotassium $\left[\mathrm{K}\left(\mathrm{C}_{3} \mathrm{H}_{5}\right)\right]$ and pyridine produce the 1,4-dihydropyridido potassium derivative exclusively in a reversible equilibrium, the isolated product is unstable in THF or pyridine. However, 
the analogous dianionic zincate, $[\mathrm{K}(18-\mathrm{crown}-6)]_{2}\left[\mathrm{Zn}\left(\mathrm{NC}_{5} \mathrm{H}_{5}-4-\mathrm{C}_{3} \mathrm{H}_{5}\right)_{4}\right](196)$ not only preserves the reversibility of the insertion process in solution, but its solid form can be stored for months under an inert atmosphere at low temperature without undergoing decomposition. Significantly, in the crystal the two [K(18-crown-6) $]^{+}$ moieties each interact with the $\left(\mathrm{NC}_{5} \mathrm{H}_{5}\right)$ ring of one amido ligand in a contact ion pair arrangement (Scheme 124).

Scheme 124. Preparation of an allyl substituted 1,4-dihydropyridido potassium complex and its more stable zincate analog (196).<smiles>C=CCC1C=CN(C=C)C=C1</smiles>

$$
\left[\mathrm{K}\left(\mathrm{C}_{3} \mathrm{H}_{5}\right)\right]
$$<smiles>c1ccncc1</smiles>

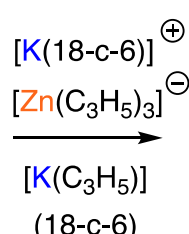<smiles>C=CCC(/C=C\C)C(C)(C)C</smiles>

$\left(18-c^{\prime}-6\right)$

(196)<smiles>[134CH3]</smiles>

Since $\mathrm{Et}_{2} \mathrm{Zn}$ is a volatile liquid, it can be used neat in reactions. Refluxing it with sodium hydroxide had a surprising outcome in producing the disodium trizinc oxo-centered cluster $\left[\mathrm{Na}_{2}\left(\mathrm{ZnEt}_{2}\right)_{3}\left(\mu_{5}-\mathrm{O}\right)\right](197) .{ }^{224} \mathrm{~A}$ mechanism has been proposed in which two molecules of initially formed EtZnONa lose $\mathrm{ZnO}$ to form $\mathrm{Na}_{2} \mathrm{OZnEt}_{2}$, which picks up two molecules of $\mathrm{Et}_{2} \mathrm{Zn}$ to form the isolated product (Scheme 125). Together with the heavier alkali metal congeners, $\mathrm{M}_{2}\left(\mathrm{ZnEt}_{2}\right)_{4}\left(\mu_{6}-\mathrm{O}\right)(\mathrm{M}=\mathrm{K}, 198$; or $\mathrm{Rb}, 199)$, this sodiumzinc species belongs to a set of compounds with extended structures comprising highly 
symmetric crystal-packing motifs supported by intercluster interactions between the alkali metal ions and $\mathrm{H}$ atoms of the ethyl groups.

Scheme 125. Proposed sequence of events leading to the formation of the disodium trizinc oxo-centered cluster $\left[\mathrm{Na}_{2}\left(\mathrm{ZnEt}_{2}\right)_{3}\left(\mu_{5}-\mathrm{O}\right)\right](\mathbf{1 9 7})$.

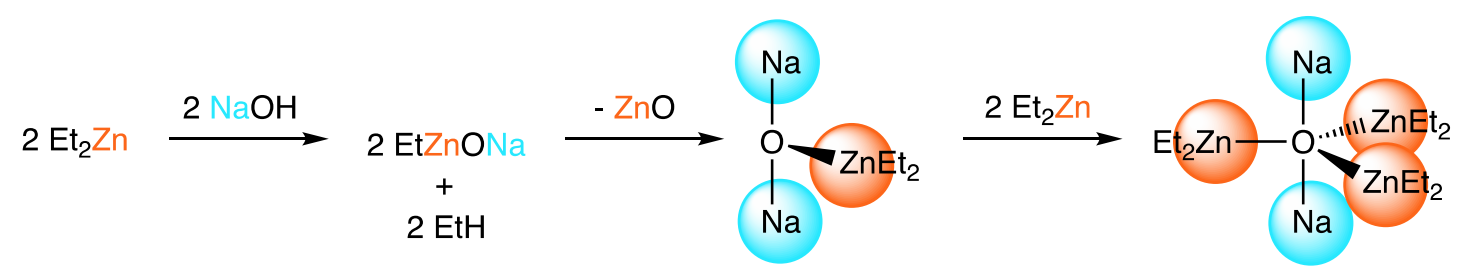

(197)

\subsection{Group 13 Chemistry}

\subsubsection{Lithium Aluminum Hydride - The Original Alkali-Metal Aluminate}

The most well-known and widely used alkali-metal aluminate complex is lithium aluminum hydride, formulated as $\mathrm{LiAlH}_{4}$ and often abbreviated further to simply LAH. Inexpensive and commercially available, $\mathrm{LiAlH}_{4}$ is a highly versatile reagent whose wide-ranging utility can generally be assigned to some sort of synergistic effect between the two main group metals, since it shows enhanced ethereal solubility compared with its homometallic lithium component $\mathrm{LiH}$ and easier handling and purification than its homometallic aluminum component $\mathrm{AlH}_{3}$. Lithium aluminum hydride was first reported by Schlesinger and co-workers in 1947 through the reaction of four equivalents of $\mathrm{LiH}$ with $\mathrm{AlCl}_{3}{ }^{225}$ Nystrom and Brown soon recognized the reducing power of $\mathrm{LiAlH}_{4}$ in organic chemistry and it is this reactivity which dominates to the present day, ${ }^{226-228}$ especially for the reduction of common functional groups such as aldehydes, ketones, esters, carboxylic acids and amides. This reducing power can be tempered by pre-reaction with pyridine, resulting in nucleophilic addition to 
form lithium tetrakis(dihydropyridyl)aluminate which is more selective in that it will reduce aldehydes or ketones in the presence of carboxylic acids or esters which remain untouched. ${ }^{229}$

More recently, the versatility of $\mathrm{LiAlH}_{4}$ has come to the fore with its application in a variety of alternative areas. For example, its relatively high hydrogen content $(10.6 \%$ by weight) has made it a compound of special interest in the field of hydrogen storage for energy purposes ${ }^{230}$ while its high abundance and low toxicity has also resulted in it being probed as an inexpensive catalytic reagent. For example, Harder and coworkers have recently demonstrated the use of $\mathrm{LiAlH}_{4}$ as a catalyst for the hydrogenation of imines and explicitly noted the cooperation of the two metals within the catalytic mechanism (Scheme 126). ${ }^{231}$

Scheme 126. Preliminary proposal for the mechanism of $\mathrm{LiAlH}_{4}$ catalyzed imine hydrogenation. 

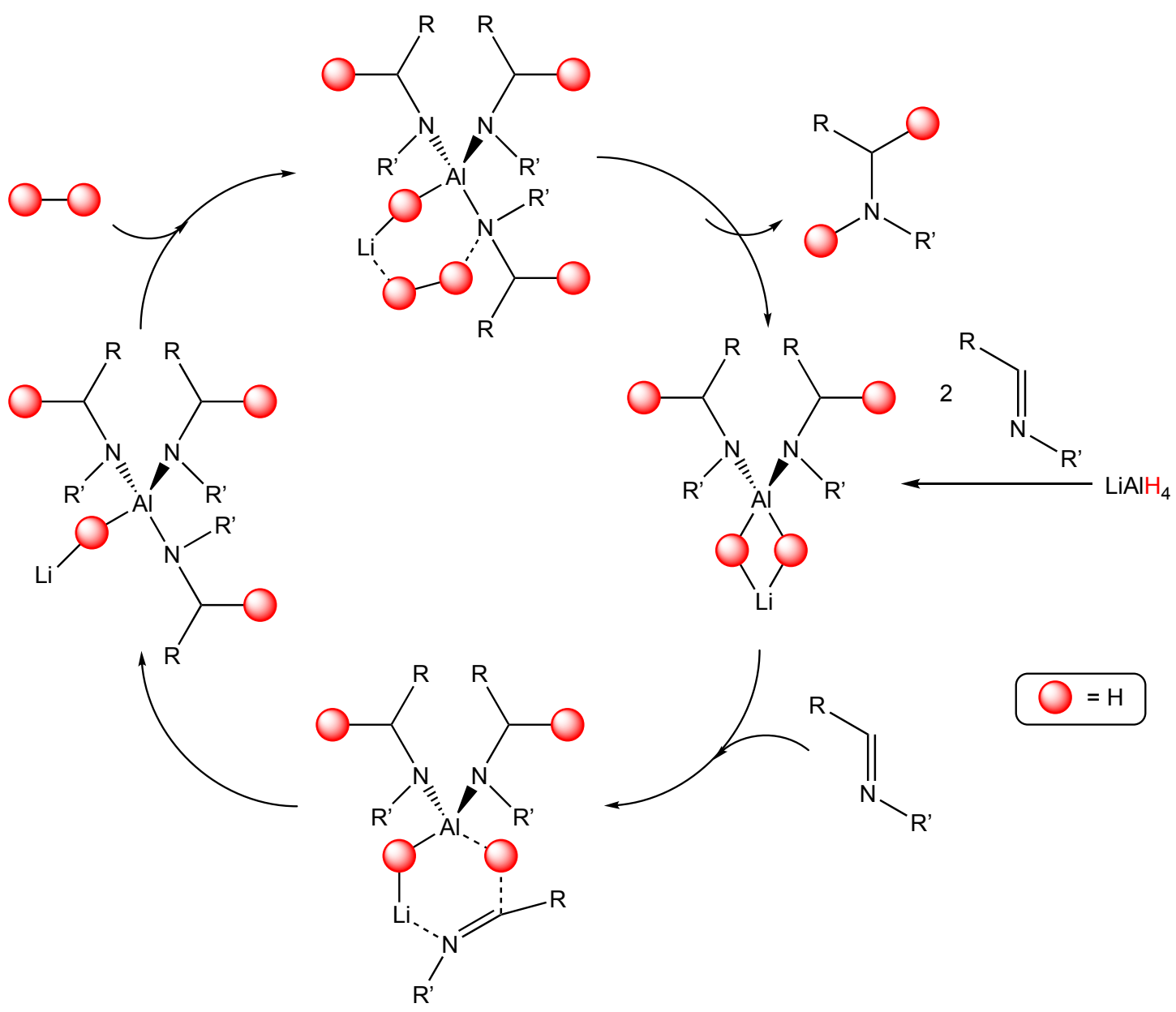

The hydroboration of alkenes has also been catalysed with $\mathrm{LiAlH}_{4}$, proceeding by initial hydroalumination of the olefin followed by $\sigma$-bond metathesis with a borane to form the borylated product and regenerating the Al-H bond. ${ }^{232}$ The scope of suitable substrates was wide, with primary, secondary and tertiary alkyl-substituted terminal alkenes all giving good conversions and excellent regioselectivity control. A variety of functional groups were tolerated (e.g. halides or silyl groups) and aryl groups with both electron-donating or electron-withdrawing groups were compatible, although the catalysis appeared to shut down for internal alkene substrates. Synergy between the metals appears a key factor here since the conversion of styrene using $\mathrm{LiAlH}_{4}$ was superior to that obtained by either neutral entity $\mathrm{AlH}_{3}$ or $\mathrm{LiH}$. 
$\mathrm{LiAlH}_{4}$ was also implicated in the catalytic hydrogenation of olefins with hydrogen gas under ambient pressure and temperature by pairing it with $\mathrm{FeCl}_{3} \cdot{ }^{233}$ Stoichiometric ratios of $\mathrm{LiAlH}_{4}$ to $\mathrm{FeCl}_{3}$ exceeding 2:1 inhibited the catalysis. While it may be tempting to presume that salt metathesis occurs to form catalytically active iron aluminum hydride species, a crystallographically characterized $\mathrm{Fe}_{2} \mathrm{LiAl}_{3} \mathrm{H}_{14}$ cluster was also identified which introduces the possibility that a more complicated trimetallic cooperativity could be occurring.

$\mathrm{LiAlH}_{4}$ has also been employed as an efficient catalyst for the dehydrocoupling of amine boranes through a combination of $\mathrm{N}-\mathrm{H}$ deprotonation, $\mathrm{B}-\mathrm{N}$ bond formation and B-N bond cleavage steps, although not all aspects of the mechanism could be resolved and the catalysis was relatively slow, presumably due to the poor solubility of the catalyst. ${ }^{234}$

Despite its prevalence in various aspects of synthetic chemistry, surprisingly little is known regarding the structural relationship between these main group metals and the simplest of anionic ligands with only a handful of dimeric (200-202), ${ }^{235-237}$ monomeric (203) ${ }^{238}$ and charge-separated (204) ${ }^{236}$ complexes characterized (Figure 33). 

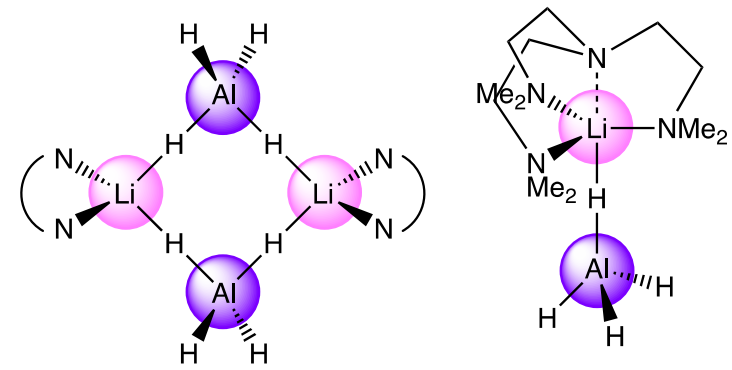

(203)

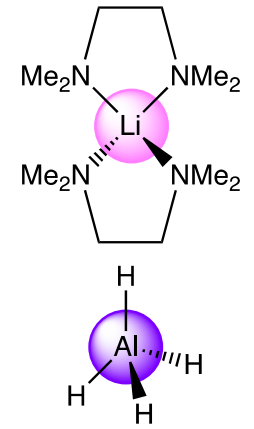

(204)

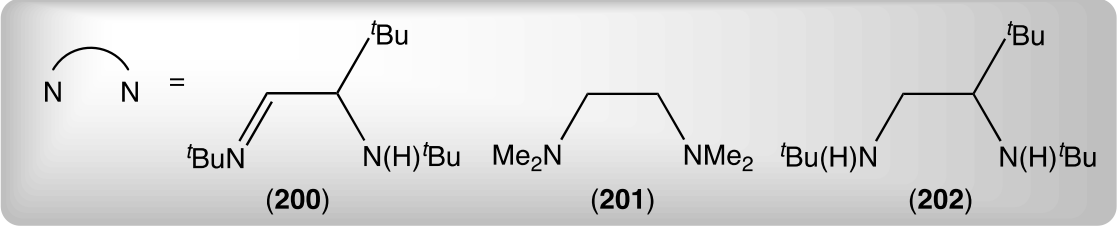

Figure 33. Crystallographically characterized examples of $\mathrm{LiAlH}_{4}$ complexes with dimeric, monomeric or charge-separated structures.

\subsubsection{Modified Forms of $\mathrm{LiAlH}_{4}$}

With four hydride groups, lithium aluminum hydride represents an excellent starting point for the development of modified lithium aluminates, through the partial or complete replacement of hydride with larger anions. This can be achieved by direct reaction of $\mathrm{LiAlH}_{4}$, using it as a Brønsted base to deprotonate a substrate with concomitant loss of $\mathrm{H}_{2}$. Recent general examples of this include the deprotonating action of $\mathrm{LiAlH}_{4}$ on alcohols, ${ }^{239}$ silanols ${ }^{240}$ and primary amines. ${ }^{241}$ This technique can be used to access highly organosoluble and water-stable weakly coordinating aluminate anions as their lithium salts, providing valuable starting materials for the preparation of catalytically active cationic transition metal complexes via salt metathesis routes 242,243 and to synthesize the lithium salt of the tris(pyrazolyl)hydroaluminate ligand, an aluminum-centered derivative of the 
corresponding boron centered "scorpionate" ligand introduced by Trofimenko in the 1960 's. ${ }^{244}$

Returning to the catalytic arena, modification of $\mathrm{LiAlH}_{4}$ for such purposes was demonstrated by its reaction with $t$-butanol, producing heteroleptic $\mathrm{LiAlH}_{2}\left(\mathrm{O}^{t} \mathrm{Bu}\right)_{2}$ (205) which could catalyse the dehydrocoupling of amine boranes ${ }^{245}$ although the catalytic performance was less efficient than either homometallic $\mathrm{AlH}_{2}\left(\mathrm{O}^{t} \mathrm{Bu}\right)$ or homoleptic $\mathrm{LiAlH}_{4}$ (vide supra) suggesting a negative synergistic effect in this instance. Similarly, $\mathrm{LiAlH}_{4}$ reacts with the bulky secondary amine $\mathrm{HMDS}(\mathrm{H})$ to yield the bisamido derivative $\mathrm{LiAl}(\mathrm{H})_{2}(\mathrm{HMDS})_{2}$ (206), which was demonstrated to be an excellent catalyst for hydroboration of aldehydes and ketones, giving high conversions in short time scales at room temperature whilst requiring only $1 \%$ catalyst loading. ${ }^{246}$ Cooperativity of the two metals is crucial as the Lewis acidic lithium grips the Lewis basic substrate and holds it in close proximity to the aluminum atom, facilitating the hydride transfer step. Furthermore, the identity of any Lewis donor coordinated to lithium plays an important role with labile THF outperforming tridentate PMDETA (scheme 127), whose chelating nature means it cannot adequately free up a coordination site on lithium for the essential substrate coordination step.

Scheme 127. Hydroboration of acetophenone with HBPin using various alkali metal HMDS-based aluminate pre-catalysts derived from 206. 


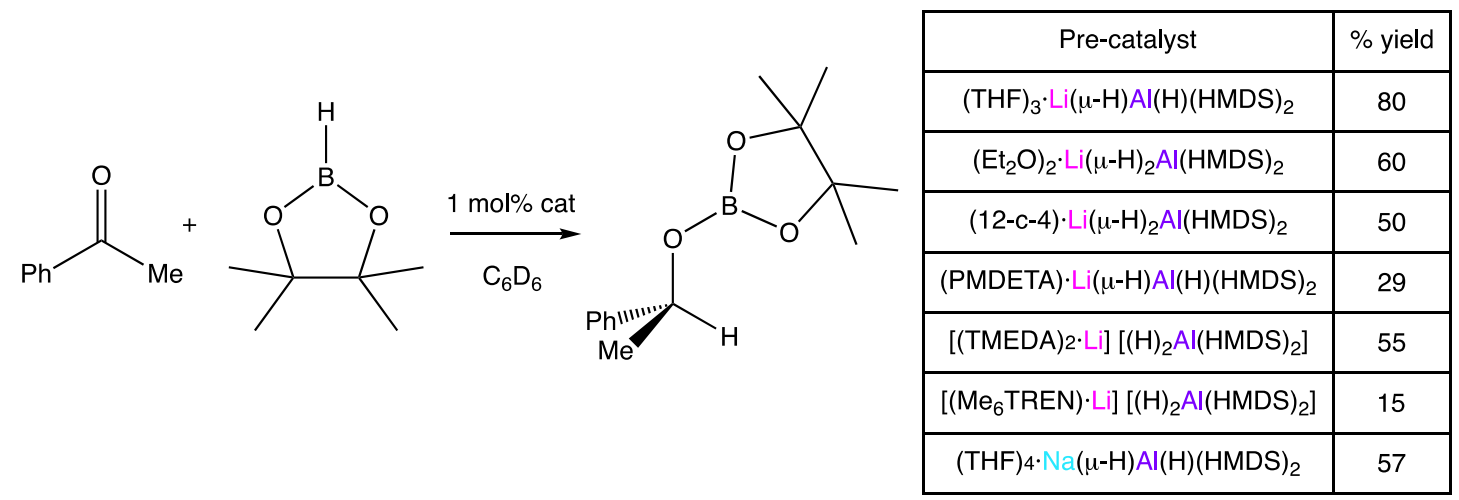

Commercially available and relatively inexpensive DIBAL-H $\left({ }^{i} \mathrm{Bu}_{2} \mathrm{AlH}\right)$ provides an alternative access point to modified hydrido-containing aluminates via a cocomplexation approach with an alkali-metal reagent. This has been established using the utility amide LiTMP to generate heterotrileptic ${ }^{i} \mathrm{Bu}_{2} \mathrm{AlTMP}(\mathrm{H}) \mathrm{Li}(\mathbf{2 0 7})$ which can be solvated by a variety of different Lewis donors resulting in a diverse range of contacted and solvent-separated structures. ${ }^{247}$ Demonstrating the versatility of these TMP-based systems, catalytic hydroboration of aldehydes, ketones and phenylacetylene, deprotonation of 1-methyl-1,2,4-triazole and addition to pyrazine were all shown to be possible (scheme 128).

Scheme 128. Flexible synthetic utility of heterotrileptic lithium aluminates based on 207, generated via cocomplexation of LiTMP with DIBAL-H. 


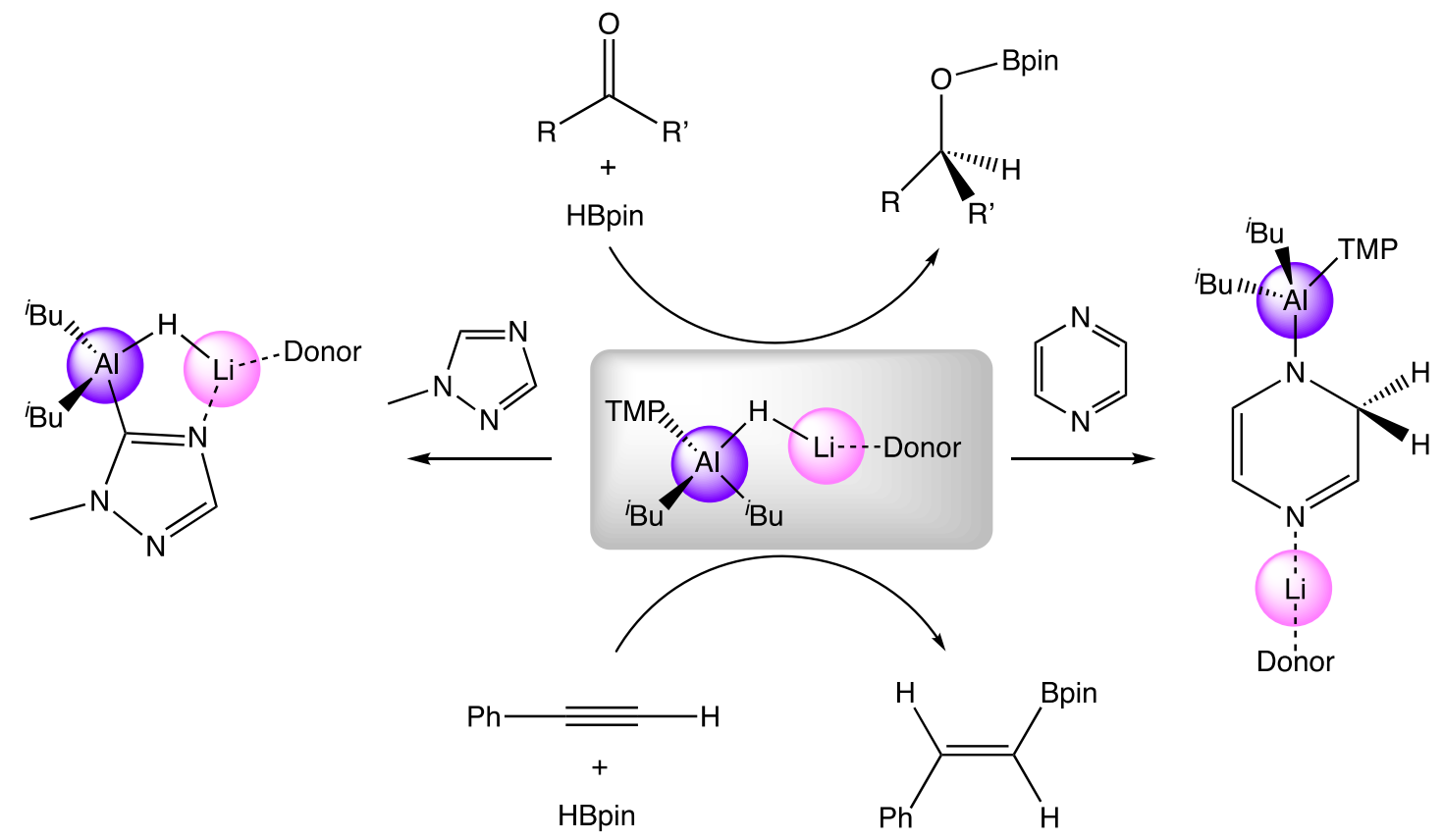

\subsubsection{Trans-metal-trapping}

Lithium aluminates provide one of the pre-eminent examples of trans-metal trapping in action, a prime example being the LiTMP/'Bu$_{2} \mathrm{Al}(\mathrm{TMP})$ pairing. ${ }^{248}$ The principle behind this chemistry is that the two metallic moieties cannot, for steric reasons, come together to form a typical bimetallic structure (in this case due mainly to the bulk of the TMP groups inhibiting formation of a Li-N-Al-N four-membered ring, scheme 129, inset). The stronger base (LiTMP) is therefore free to execute a "traditional" metalation of a substrate to form the amine TMP(H) and a new lithium carbanion whose diminished steric profile with respect to LiTMP, coupled with the strong carbophilicity of the second metal (aluminum), means that the two metallic species can now conjoin to give a bimetallic species containing the deprotonated substrate. The implications of this coming together of the two metallic moieties are two-fold: the secondary metal (in this case aluminum) now binds to the reactive carbanion giving a less-polar, sedated $\mathrm{M}-\mathrm{C}$ bond whilst simultaneously removing the lithium carbanion from the mixture, meaning that even if the position of equilibrium 
of the lithiation reaction lies heavily in favor of the starting materials the reaction can now proceed virtually quantitatively (scheme 129). Despite the two metallic species not coming together in the first instance there is still clear synergy at play here as the reaction cannot advance in any meaningful way without the two metals working in concert with one another.

Scheme 129. General concept behind trans-metal-trapping with LiTMP the base and ${ }^{i} \mathrm{Bu}_{2} \mathrm{Al}(\mathrm{TMP})$ the trap. Metalation is only efficient when the trap sees the emerging carbanion and drives the equilibrium to the desired product.

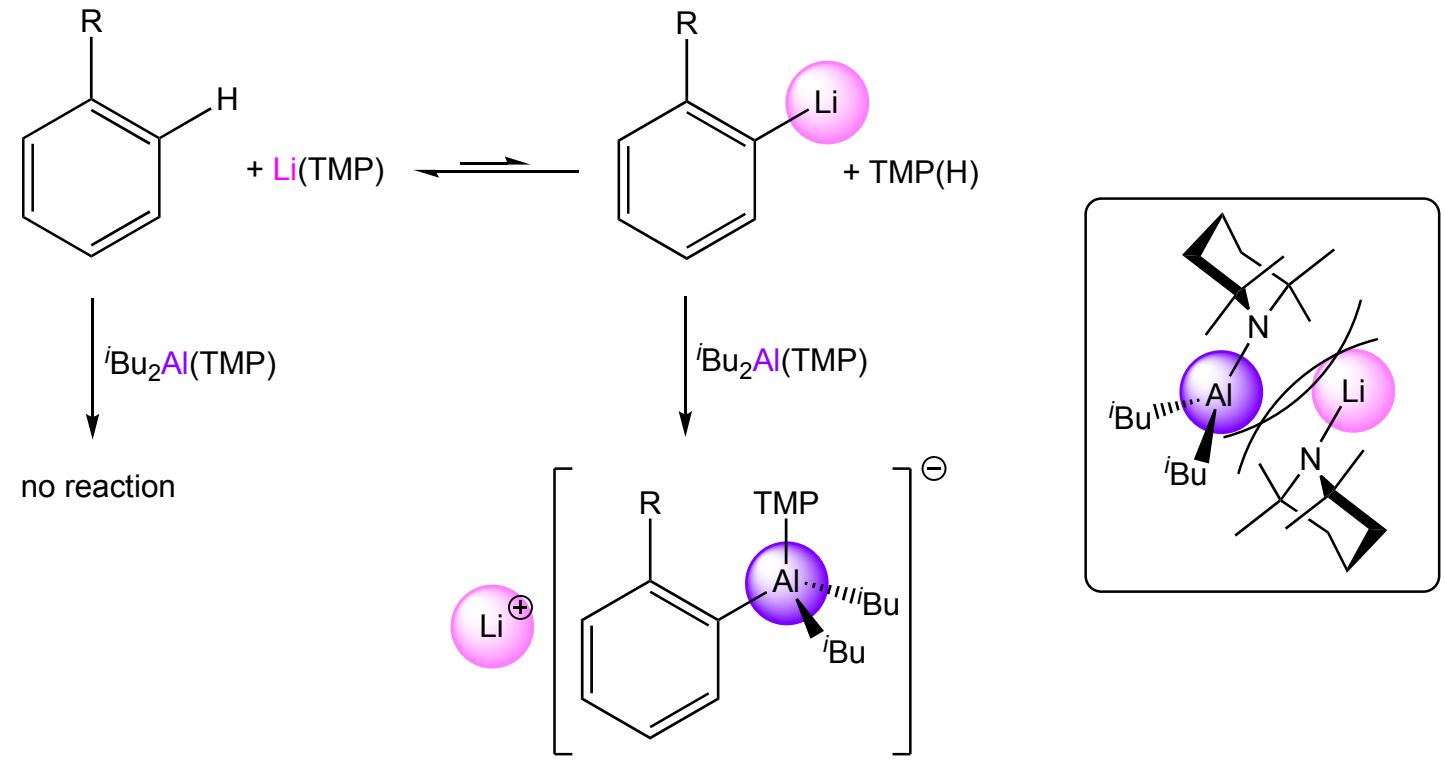

Trans-metal-trapping using the LiTMP/'BB ${ }_{2} A$ AITMP tandem has been shown to work for both aryl and alkyl substrates and is halogen tolerant as demonstrated by the preference for ortho-metalation of 4-haloanisoles over metal-halogen exchange. ${ }^{249}$ This approach, coupled with alternating electrophilic quenching steps, provides facile access to hetero-halogenated aromatic compounds (scheme 130). 
Scheme 130. Exemplar of halogen-tolerant trans-metal-trapping starting from 4haloanisoles.

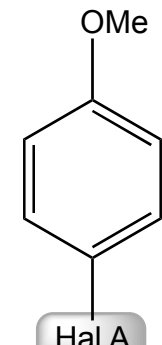

Hal A i) $L i(T M P)$ $\stackrel{{ }^{i} \mathrm{Bu}_{2} \mathrm{Al}(\mathrm{TMP})}{\longrightarrow}$

ii) halogenating agent<smiles>CCCc1cc(C)cc(CCC)c1OC</smiles>

halogenating agent $=\mathrm{I}_{2}, \mathrm{NBS}, \mathrm{SO}_{2} \mathrm{Cl}_{2}$

Such methodology can be extended to ferrocene, with a stoichiometry dependence influencing the final product. ${ }^{173}$ When carried out in a 1:1 ratio of ferrocene to metal reagents, one of the cyclopentadienyl rings is metalated and the ferrocenyl anion is consequently trapped within the bimetallic framework while in a 1:2 ratio, both rings are metalated with the structure displaying staggered $\mathrm{Al}-\mathrm{C}$ units to minimize steric clashing of the bimetallic units (akin to zincate structures 125 and 126). Despite the capability of bimetallic bases to synergistically tetrametalate group 8 metallocenes having being demonstrated previously, ${ }^{61,250}$ no further reactivity beyond dimetalation was noted even when further equivalents of LiTMP/iBu${ }_{2} A$ ITMP were employed. This was rationalized by the generation of non-volatile, Lewis basic TMP(H) from the deprotonation reaction, which subsequently solvates the lithium atom and prevents the formation of the inverse crown ring required to stabilize a tetrametalated ferrocenyl ring.

The importance of the steric bulk provided by both components of a trans-metaltrapping tandem through their TMP anions is clearly demonstrated in the reaction of lithium/aluminum pairings with common Lewis donors such as THF. Sterically less 
inhibited $\mathrm{Al}^{\prime} \mathrm{Bu}_{3}$ will form a co-complex with LiTMP which can subsequently be solvated at lithium by THF without any disruption to the cycloether molecule (208). ${ }^{251}$ However, using sterically bulkier monomeric ${ }^{i} \mathrm{Bu}_{2} \mathrm{AlTMP}{ }^{252}$ prohibits the formation of the analogous co-complex with the strongly basic LiTMP molecule rather metalating THF in the $\alpha$-position (scheme 131).

Scheme 131. Contrasting reactions of lithium-aluminum TMP systems with contacted system in aluminate acting as a Lewis acid to THF; whereas the separated system acts as a lithiating base to THF through trans-metal-trapping.

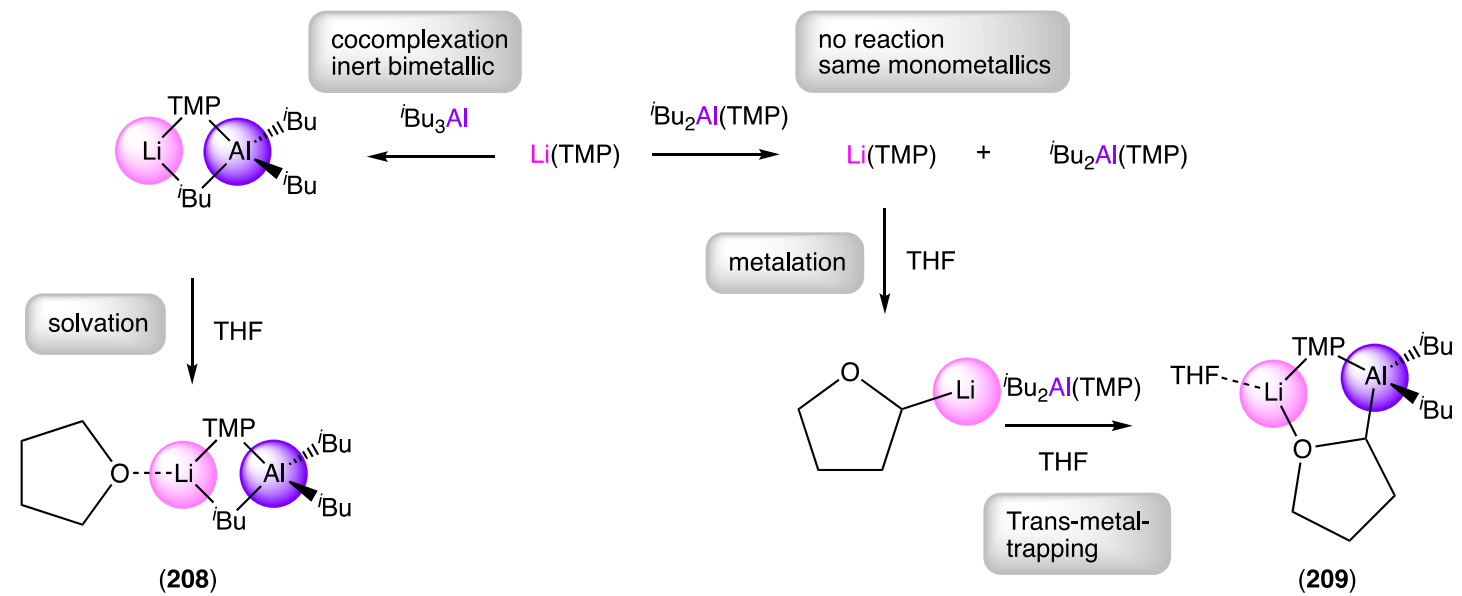

Rather than progress down the typical decomposition route of lithiated THF through ring opening to yield ethene and the lithium enolate of acetaldehyde, ${ }^{177}$ the metalated ring is trapped intact by the bulky aluminum reagent to yield $\mathrm{Li}(\mu-\mathrm{TMP})\left(\mu-\mathrm{OC}_{4} \mathrm{H}_{7}\right) \mathrm{Al}^{\mathrm{i}} \mathrm{Bu} \mathrm{u}_{2}$ (209). ${ }^{253}$ This is not limited to THF, with a wide variety of bi- and tri-dentate N/O donors also undergoing metalation and trapping with the LiTMP/'B $\mathrm{B}_{2} \mathrm{AlTMP}$ pair whilst simply solvating the related $\mathrm{LiTMP} / \mathrm{Al}^{\mathrm{B}} \mathrm{Bu}_{3}$ co-complex. ${ }^{254}$

An interesting divergence in reactivity has been witnessed for $\mathrm{LiNEt}_{2}$ and $\mathrm{LiPPh}_{2}$ with (pyrrolylaldiminato)methylaluminum chloride. ${ }^{255}$ Performed in a stoichiometric 2:1 
ratio, the lithium amide underwent simultaneous insertion (nucleophilic addition) across the $\mathrm{C}=\mathrm{N}$ functionality and salt metathesis (to replace the $\mathrm{Cl}$ ligand with $\mathrm{NEt}_{2}$ ), yielding 210. While one equivalent of the lithium phosphide also inserted into the $C=N$ bond, the second equivalent attacked and ring opened THF, with the resultant $\mathrm{LiO}\left(\mathrm{CH}_{2}\right)_{4} \mathrm{PPh}_{2}$ unit undergoing salt metathesis at the $\mathrm{Al}-\mathrm{Cl}$ bond to generate an aluminoxide unit (211, scheme 132). This ring-opening of THF seems to be synergistically influenced since $\mathrm{LiPPh}_{2}$ is commercially available as a THF solution, suggesting that any ring opening by this homometallic reagent is negligible, although we note that only a small conversion is necessary if the resultant anion is being trapped in a TMT process.

Scheme 132. Divergent reactivity of amide LiNEt 2 and phosphide $\mathrm{LiPPh}_{2}$ towards (pyrrolylaldiminato)methylaluminum chloride.

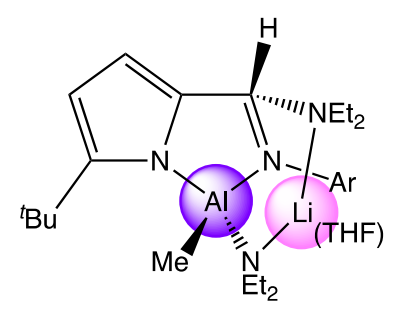

(210)
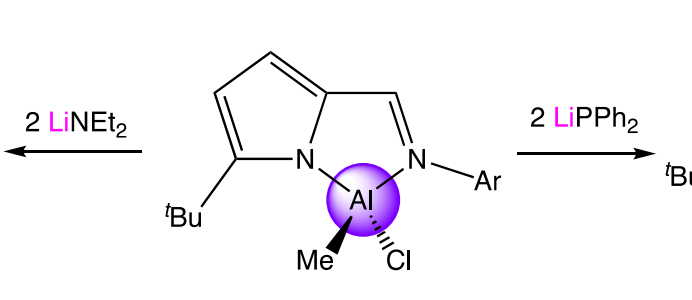

(211)

Normally considered as robust neutral ligands, $\mathrm{N}$-heterocyclic carbenes (NHC) have recently attracted increasing attention with regards to their main group coordination chemistry (see also section 2.4.2). ${ }^{256}$ The action of strong main group bases such as $n$ butyllithium has provided access to the class known as abnormal NHC complexes, whereby the backbone of the imidazole ring is deprotonated formally resulting in formation of an anionic dicarbene with both the deprotonated C4 and the 'normal' C2 
(of an adjacent molecule) both bonding to the lithium cation. ${ }^{257}$ Another LiTMP/'Bu ${ }_{2}$ AlTMP trans-metal-trapping strategy has been employed with the saturated carbene 1,3-bis(2,4,6-trimethylphenyl)-4-5-dihydroimidazol-2-ylidene (represented as SIMes on account of its Saturated Imidazole ring and Mesityl exocyclic constituents). Rather than attack the NHC backbone, one of the aromatic bound methyl groups is metalated, with the aluminum reagent trapping the resulting carbanion to yield an aluminate center while the lithium migrates to the 'normal' C2 carbene position in 212 (scheme 133). ${ }^{258}$ An alkyllithium base on its own forces ring opening of the carbene ring (213), with the mechanism believed to involve metalation of the aromatic bound methyl group prior to ring cleavage, emphasising the synergistic relationship between lithium and aluminum in the metalation of SIMes.

Scheme 133. Trans-metal-trapping of the saturated NHC SIMes leading to alumination of a methyl arm of the mesityl substituent (212) versus conventional lithiation leading to ring opening of the $\mathrm{NHC}$ ring (213). 


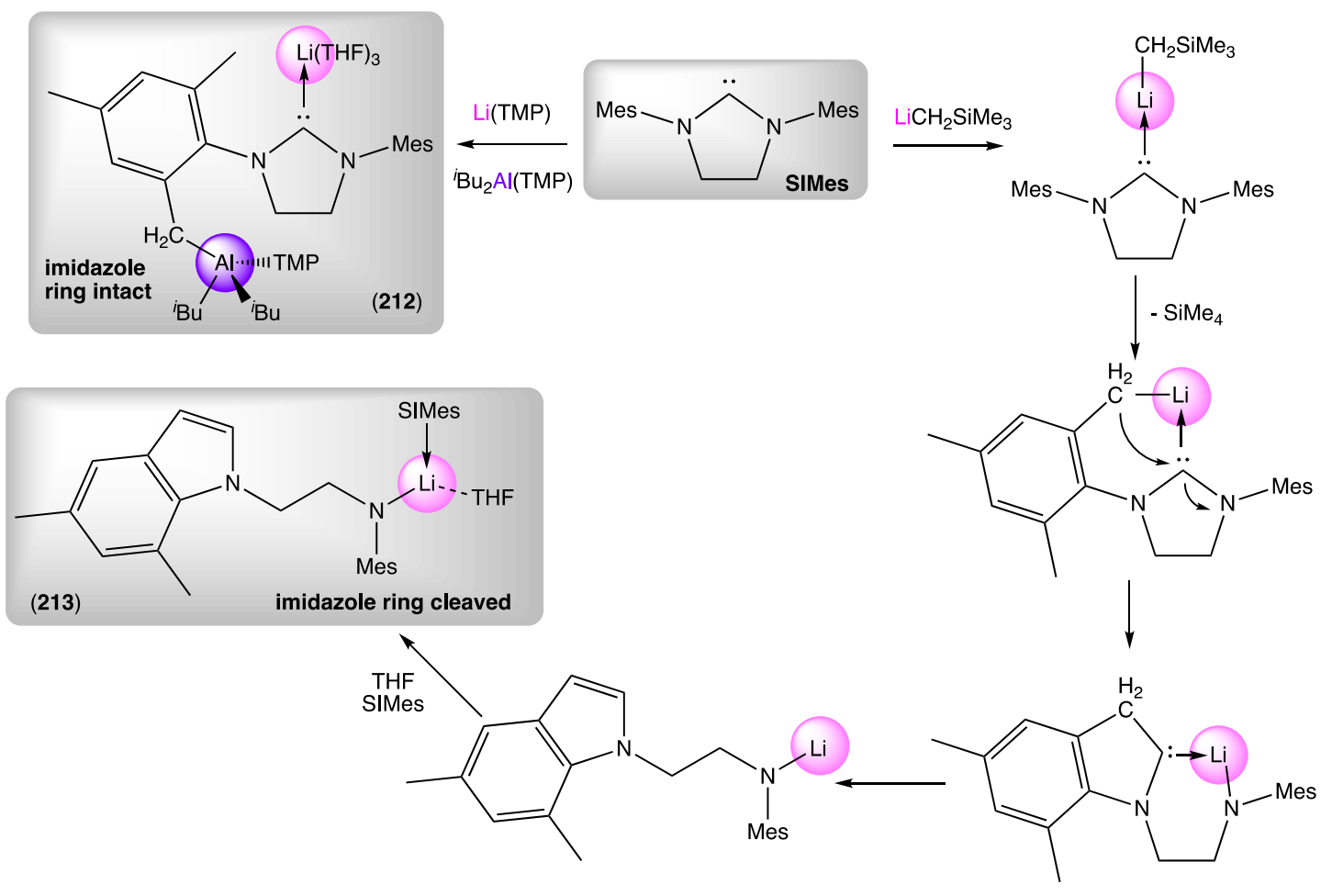

The benefits of utilizing a trans-metal trap for structurally identifying otherwise fleeting reaction intermediates is witnessed through the utilization of 1-phenyl- $1 \mathrm{H}$ benzotriazole as the substrate. This multinitrogen heterocyclic substrate is susceptible to deprotonation at two competing sites, as evidenced by the reaction with 0.5 equivalents of $\mathrm{ZnCl}_{2} \cdot T M E D A / 1.5$ LiTMP and subsequent electrophilic quenching with iodine, which shows a mixture of two mono-iodinated as well as some di-iodinated compounds. ${ }^{190}$ LiTMP on its own produces a less stable metalated intermediate resulting in ring opening with loss of molecular nitrogen and $\mathrm{C}-\mathrm{C}$ bond formation to form an unusual dianion (214); whereas in the presence of the aluminum centered trap two isomeric products were identified (215 and 216). ${ }^{259}$ In each case the products were dimeric complexes containing a mono-metalated substrate with the difference residing in the site of deprotonation (scheme 134). 
Scheme 134. Lithiation and trans-metal-trapping of 1-phenyl-1H-benzotriazole leading to its rearrangement and loss of $\mathrm{N}_{2}$, compared to simple ortho-metalations respectively.
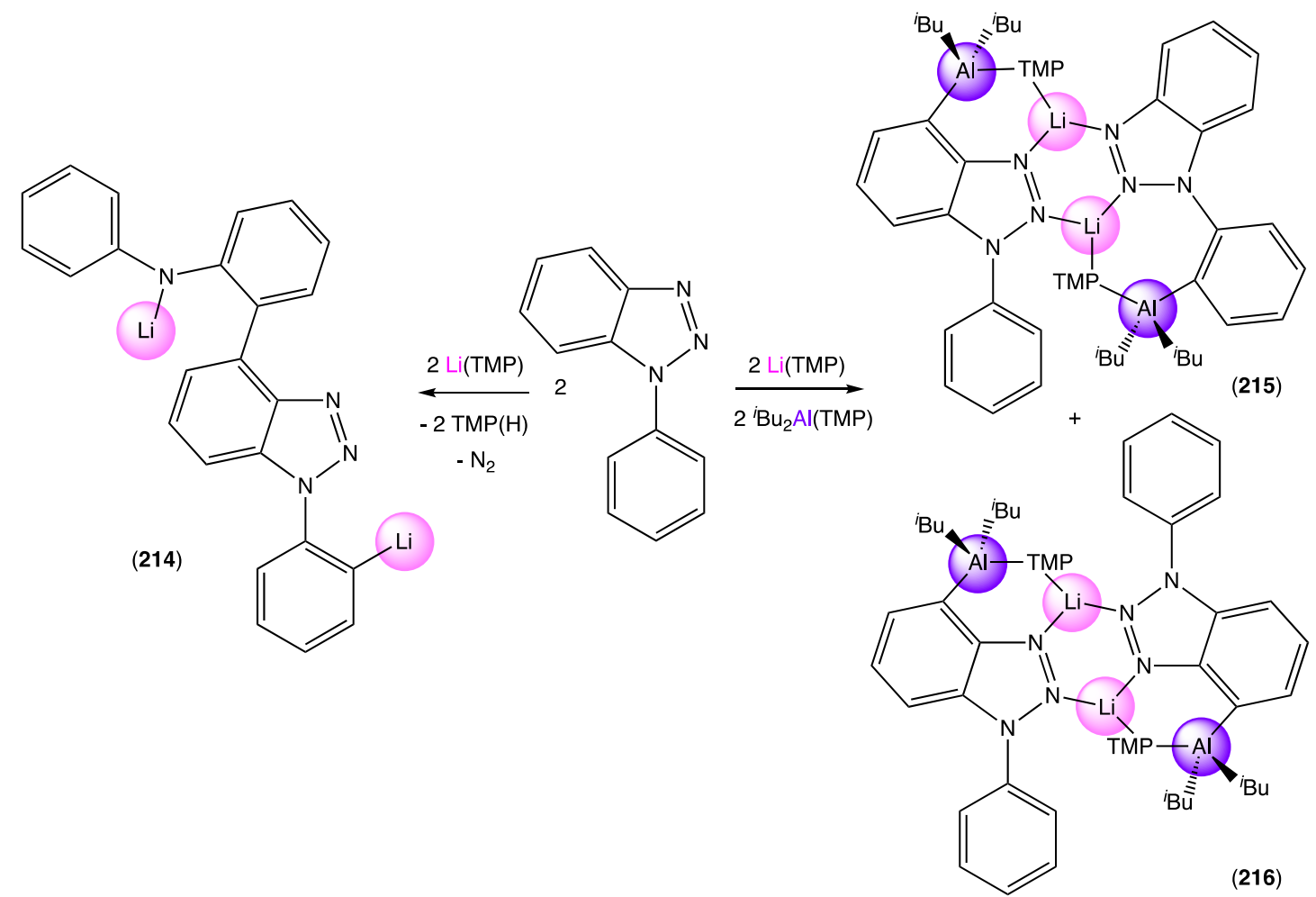

Metalation of $N, N$-dimethylphenylethylamine at the benzylic position produces a product which is primed to extrude styrene and dimethylamido-metal via a $\beta$ elimination process. In the presence of ${ }^{i} \mathrm{Bu}_{2} \mathrm{AITMP}$ and THF, the $\mathrm{Me}_{2} \mathrm{NLi}$ cleaved from the substrate post-lithiation with LiTMP can be captured in a bimetallic framework $(217) .{ }^{179}$

Scheme 135. Lithiation of $N, N$-dimethylphenylethylamine leading to $\beta$-elimination with the resulting sterically unencumbered lithium amide trapped by ${ }^{i} \mathrm{Bu}_{2} \mathrm{AlTMP}$ in bimetallic framework (217). 

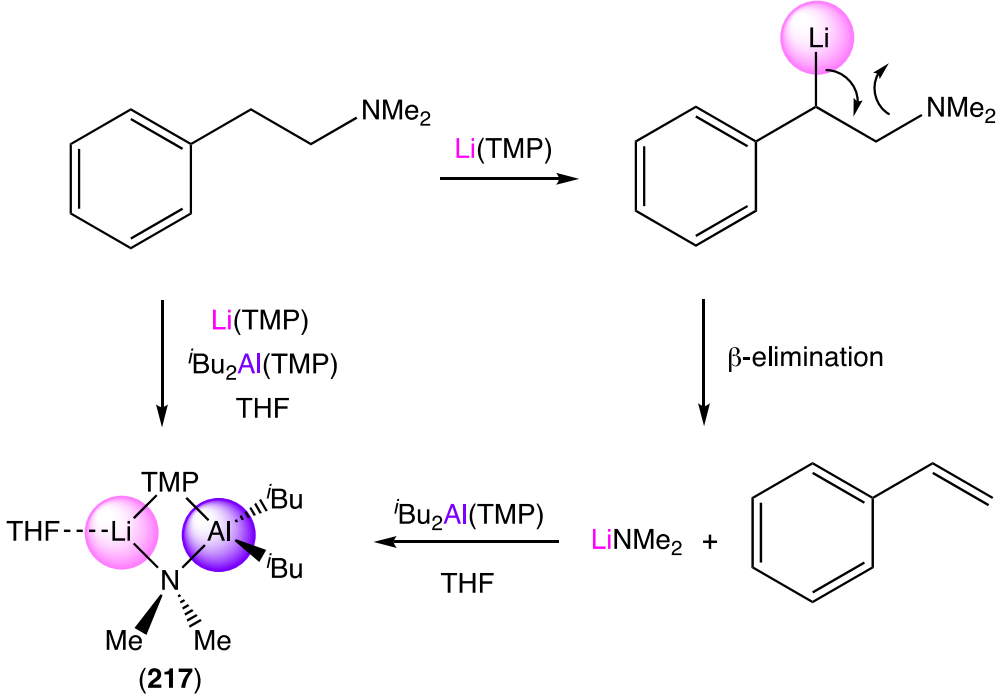

Moving down one place in group 13, gallium has also been proven to operate as an efficient trapping agent. In particular, the tris-silylalkyl compound $\mathrm{Ga}\left(\mathrm{CH}_{2} \mathrm{SiMe}_{3}\right)_{3}$ has been utilized, again with LiTMP as the active base. ${ }^{260}$ Using pyrazine as the substrate, the synergy at play between the two metals and the importance of steric bulk was clearly evident. Employing the homoleptic bimetallic gallate reagent $\mathrm{LiGa}\left(\mathrm{CH}_{2} \mathrm{SiMe}_{3}\right)_{4}$ (218), only 1,2-addition across the NC bond of pyrazine was witnessed, yielding 219. However, upon switching to the LiTMP/Ga $\left(\mathrm{CH}_{2} \mathrm{SiMe}_{3}\right)_{3}$ pairing (in the presence of the tridentate Lewis donor PMDETA), the pyrazine substrate could be mono- or dimetalated depending on the stoichiometric ratio of metal reagents added (220 and 221 respectively, Scheme 136). Crucially, the carbanion is directly bound to the more carbophilic gallium, with the lithium lying remote to this site being coordinated by the heterocycle nitrogen atom and the PMDETA molecule.

Scheme 136. Divergent reactivity of lithium/gallium pairings towards pyrazine. 


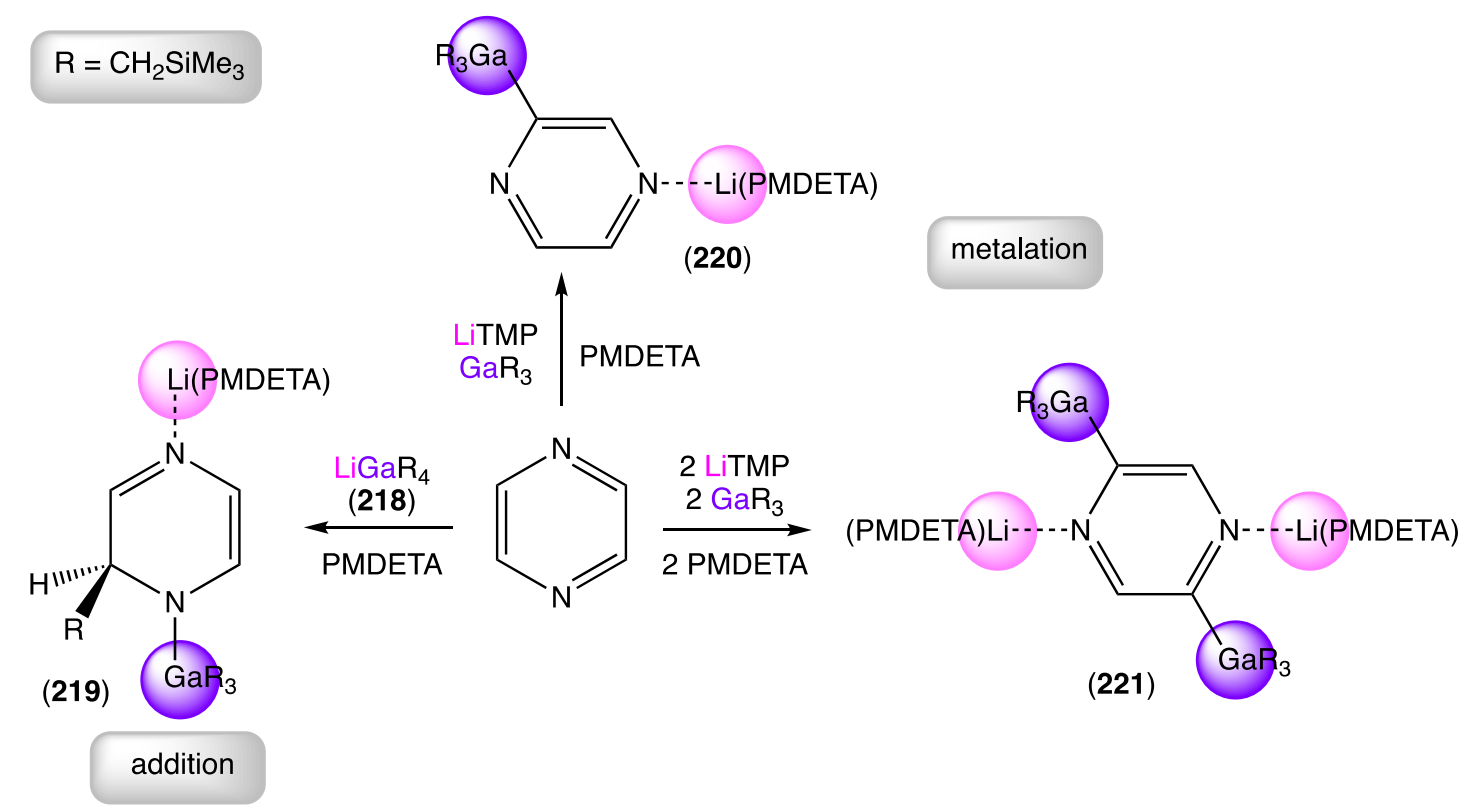

This protocol could be extended to other heterocycles such as pyridazine, pyrimidine and benzothiazole, which under conventional metalation protocols are prone to ring opening decomposition. Gallium provides an added advantage over aluminum in the metalation of sensitive fluoroaromatic compounds such as 1,3,5-fluorobenzene. ${ }^{261}$ When the resulting 2-carbanion is trapped with aluminum, it is susceptible to decomposition via benzyne formation due to elimination of an aluminum fluoride complex. However, a $\mathrm{Ga}\left(\mathrm{CH}_{2} \mathrm{SiMe}_{3}\right)_{3}$ trap allows stabilization of this carbanion in the form of a lithium gallate, meaning that the aromatic structure remains intact and is available for onward functionalization through palladium catalysed cross-coupling (Scheme 137).

Scheme 137. Trans-metal-trapping of 1,3,5-fluorobenzene and acylation of the deprotonated gallated intermediate. 
<smiles>Fc1cc(F)cc(F)c1</smiles><smiles>O=C(c1ccccc1)c1c(F)cc(F)cc1F</smiles>

$79 \%$ yield

\subsubsection{Miscellaneous Examples}

An interesting lithium aluminate complex was prepared by the deprotonation of the alcohol $\mathrm{HOC}\left(\mathrm{CH}_{2}\right)_{5} \mathrm{CH}_{2} \mathrm{~N}(\mathrm{Me}) \mathrm{CH}_{2} \mathrm{CH}_{2} \mathrm{NMe}_{2}\left(\mathrm{HOR}^{\prime}\right)$ with RAlMe $2(\mathrm{R}=\mathrm{Me}, \mathrm{Cl})$ followed by cocomplexation with ${ }^{n} \mathrm{BuLi}(\mathrm{R}=\mathrm{Me})$ or cocomplexation/salt metathesis with two equivalents of ${ }^{n} \mathrm{BuLi}(\mathrm{R}=\mathrm{Cl})$ to give $\operatorname{LiAl}\left(\mathrm{OR}^{\prime}\right)(\mathrm{Me})_{x}\left({ }^{n} \mathrm{Bu}\right)_{3-\mathrm{x}}(\mathrm{R}=\mathrm{Me}, \mathrm{x}=2, \mathbf{2 2 2} ; \mathrm{R}=\mathrm{Cl}, \mathrm{x}$ $=1$, 223) $\cdot{ }^{91}$ The presence of the $\mathrm{N}(\mathrm{Me}) \mathrm{CH}_{2} \mathrm{CH}_{2} \mathrm{NMe}_{2}$ arm provided an intramolecular Lewis basic solvating source akin to TMEDA (note the alcohol was originally prepared by deprotonating a TMEDA arm and adding this across the ketone functionality of cyclohexanone) but this was not sufficient to provide access to discrete molecular bimetallic species, with polymerization occurring via $\mathrm{CH}_{3}$ bridges. These complexes were utilized as catalysts in the Meerwein-Ponndorf-Verley reaction of benzaldehyde and isopropanol. Conversions to benzyl alcohol and acetone were not as high as when using a related lithium magnesiate complex (90, section 2.2 .6$)$ and were similar to those obtained with the homoleptic magnesium complex $\mathrm{Mg}\left(\mathrm{OR}^{\prime}\right)_{2}(\mathbf{8 9})$.

Bimetallic alkali-metal aluminates have been generated by reaction of a phosphorus/aluminum Frustrated Lewis Pair (FLP) complex with alkali-metal hydrides. ${ }^{262}$ This FLP is capable of solubilizing metal hydrides $\mathrm{MH}(\mathrm{M}=\mathrm{Li}, \mathrm{Na}, \mathrm{K})$ and capturing them within a contacted ion-pair structure (224-226) in moderate yields with the sodium derivative $\mathbf{2 2 5}$ then effective in phase-transfer catalysis for 
converting chlorosilanes to hydrosilanes (scheme 138). The synergistic influence of the aluminum moiety within the bimetallic framework is emphasized by the unreactive nature of insoluble polymeric $\mathrm{MH}$ on its own with $\mathrm{ClSiR}_{3}$.

Scheme 138. Proposed catalytic cycle for the conversion of chlorosilanes to hydrosilanes.

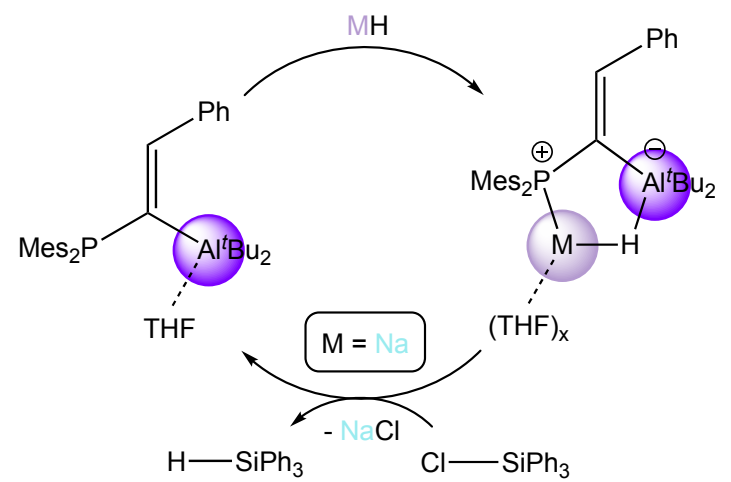

\begin{tabular}{|c|c|c|c|}
\hline $\mathbf{M}$ & $\mathbf{x}$ & $\mathbf{t}$ & yield \% \\
\hline $\mathrm{Li}(\mathbf{2 2 4})$ & 2 & $15 \mathrm{~h}$ & 40 \\
\hline $\mathrm{Na}(\mathbf{2 2 5})$ & 1 & $168 \mathrm{~h}$ & 40 \\
\hline $\mathrm{K}(\mathbf{2 2 6})$ & 4 & $48 \mathrm{~h}$ & 45 \\
\hline
\end{tabular}

Utilizing the potassium amide $\operatorname{KN}\left(\mathrm{Ar}^{*}\right)\left(\mathrm{SiMe}_{3}\right) \quad\left[\mathrm{Ar}^{*}=4\right.$-methyl-2,6bis(diphenylmethyl)phenyl] in a cocomplexation reaction with $\mathrm{AlH}_{3}$ has led to the successful synthesis of a hydride rich potassium aluminate complex in $\mathrm{KAlH}_{3} \mathrm{~N}\left(\mathrm{Ar}^{*}\right)\left(\mathrm{SiMe}_{3}\right)$ (227) which dimerized via bridging hydrides. ${ }^{263}$ This complex displayed indirect synergy in that it provided a starting point for access to homometallic $\mathrm{All}_{2} \mathrm{~N}\left(\mathrm{Ar}^{*}\right)\left(\mathrm{SiMe}_{3}\right)$ (229) via $\mathrm{AlH}_{2} \mathrm{~N}\left(\mathrm{Ar}^{*}\right)\left(\mathrm{SiMe}_{3}\right)$ (228), the desired di-iodo aluminum complex itself not being directly accessible by reaction of $\mathrm{KN}\left(\mathrm{Ar}^{*}\right)\left(\mathrm{SiMe}_{3}\right)$ with $\mathrm{All}_{3}$.

Scheme 139. Synthesis of a homometallic aluminium amide (229) via bimetallic potassium aluminate precursor (227). 


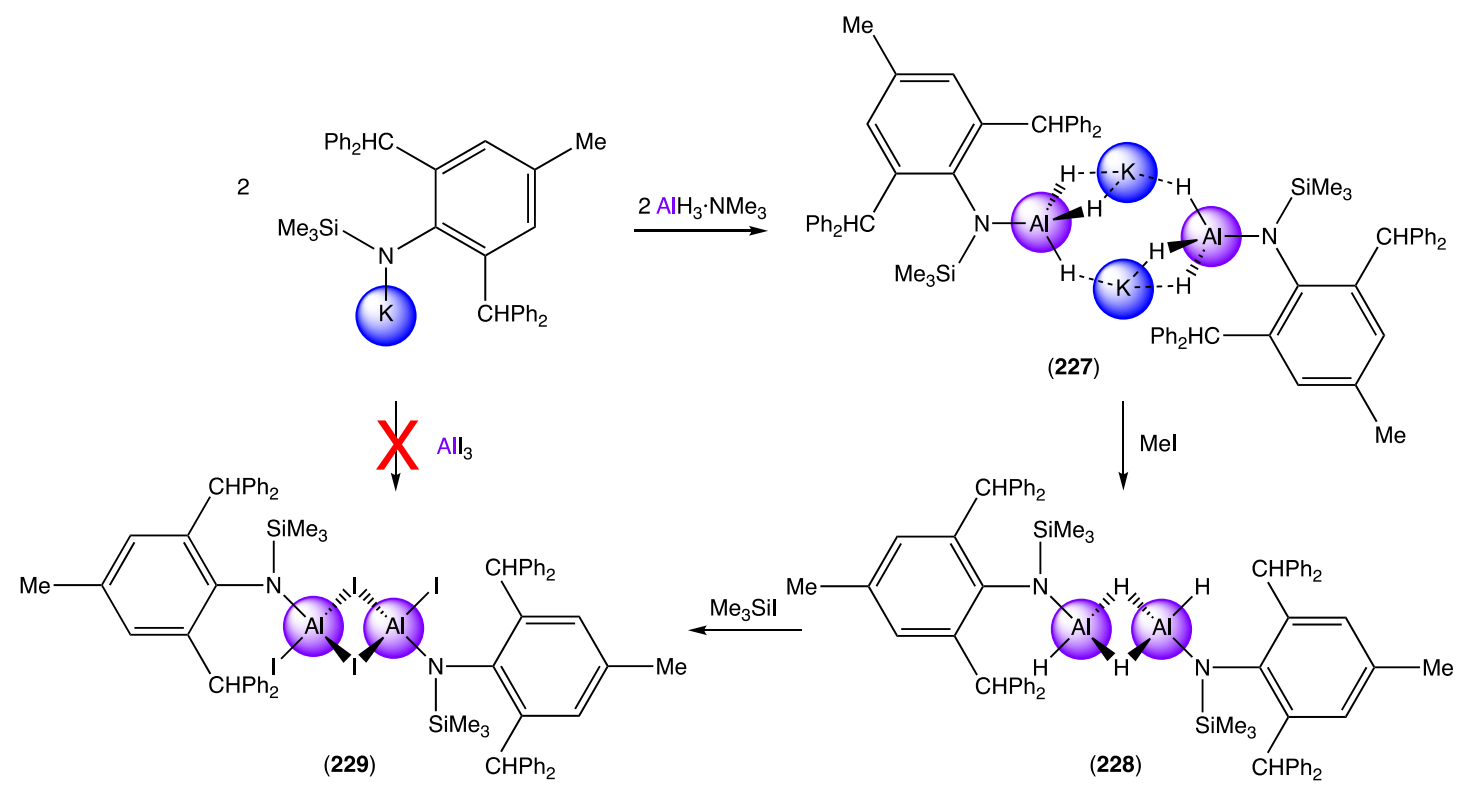

A similar route was used to form the desired aryl aluminum dihydride $\left[\mathrm{ArAlH}_{2}\right]_{2}$, first co-complexing $\operatorname{LiAr}$ [Ar = 2,6-bis(2,4,6-triisopropylphenyl)-3,5-diisopropyl-phenyl] with $\mathrm{AlH}_{3}$ to give $\left[\mathrm{LiAlH}_{3} \mathrm{Ar} \cdot \text { donor }\right]_{\mathrm{x}}\left(\right.$ donor $=\mathrm{OEt}_{2}, \mathrm{x}=1$, 230; no donor, $\mathrm{x}=2,231$ ) and then reacting with $\mathrm{Me}_{3} \mathrm{SiCl}$ to eliminate $\mathrm{LiCl}$ and $\mathrm{Me}_{3} \mathrm{SiH}^{264}$ The resulting alane subsequently reacted as a preferential hydroaluminating agent with terminal alkynes rather than as a base. ${ }^{265}$

A protocol has been developed for the preparation of lithium trispyridyl aluminate complexes with substituents at the pyridyl 6-position (e.g. 232). ${ }^{266}$ These aluminates can be utilized for rapid determination of enantiomeric excess of chiral alcohols. ${ }^{267}$ Reaction with the alcohol results in deprotonation via pyridyl basicity to yield $\mathbf{2 3 3}$, with the ${ }^{1} \mathrm{H}$ NMR chemical shift of the methyl arm at the 6-position of the pyridyl ring being sensitive to the chirality of the alcohol (scheme 140). Simple integration of these methyl resonances provides accurate information on the quantity of each enantiomer in the alcohol. A synergistic effect is clearly at play here since this approach could not be adopted by a homometallic aluminum derivative (since it would be unlikely to 
deprotonate the alcohol) or with a monovalent lithium complex as a multi-anionic system is necessary (namely the chiral alkoxide and the pyridyl anion which provides the remote NMR reporter group). These lithium aluminate complexes also find use as precursors to sandwich compounds by a salt metathesis reaction with a divalent metal halide salt. ${ }^{268}$

Scheme 140. Deprotonation of chiral alcohol with lithium aluminate 232 produces dimer 233 with close proximity of Me groups to chiral centre, allowing ee determination by integration of Me group resonances in NMR spectrum.

2

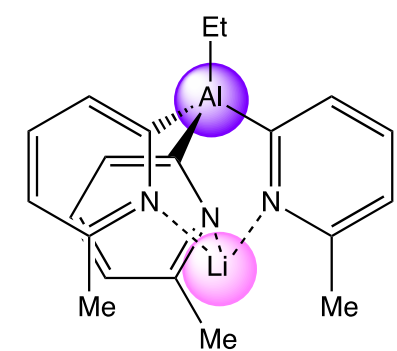

(232)

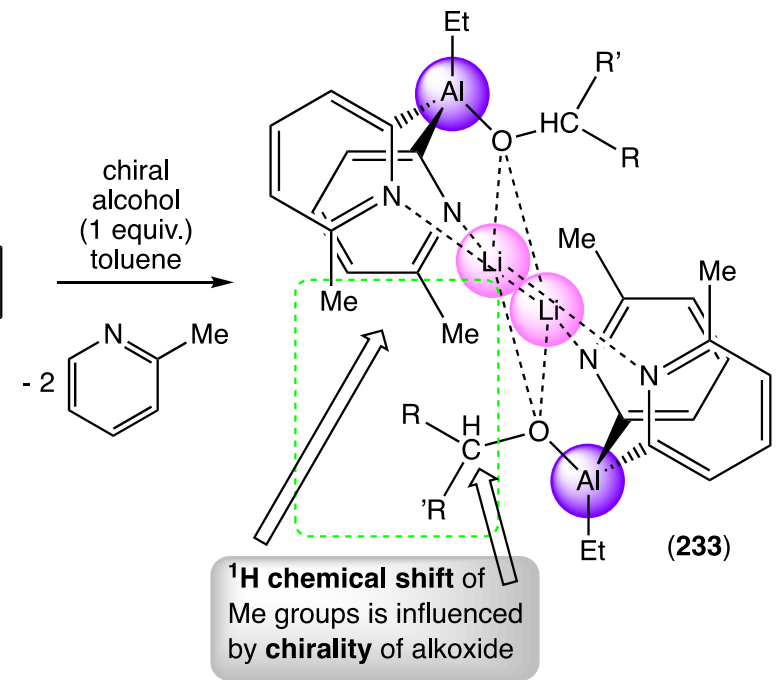

Cocomplexation of a potassium terpene oximate with an organoaluminum reagent $\left(\mathrm{AlR}_{3}, \mathrm{R}=\mathrm{Me}, \mathrm{Ph}\right)$ produced dimeric potassium aluminate species $\mathbf{2 3 4}$ and $\mathbf{2 3 5}$ which could deprotonate a ketone at the $\alpha$-carbon (generating an enolate) rather than undergoing addition across the unsaturated functional group (scheme 141). ${ }^{269}$ Interestingly, when $\mathrm{R}=\mathrm{Me}$, the bimetallic complex operated via alkyl basicity yet when $\mathrm{R}=\mathrm{Ph}$, the system switched to oximate basicity. The synergistic effect inherent in this system, alongside the key role played by the bio-based oximate ligand, were demonstrated via DFT calculations. 
Scheme 141. Cocomplexation synthesis of a potassium aluminate base (molecular structure of $\mathbf{2 3 4}$ in inset) and its reaction with a ketone.
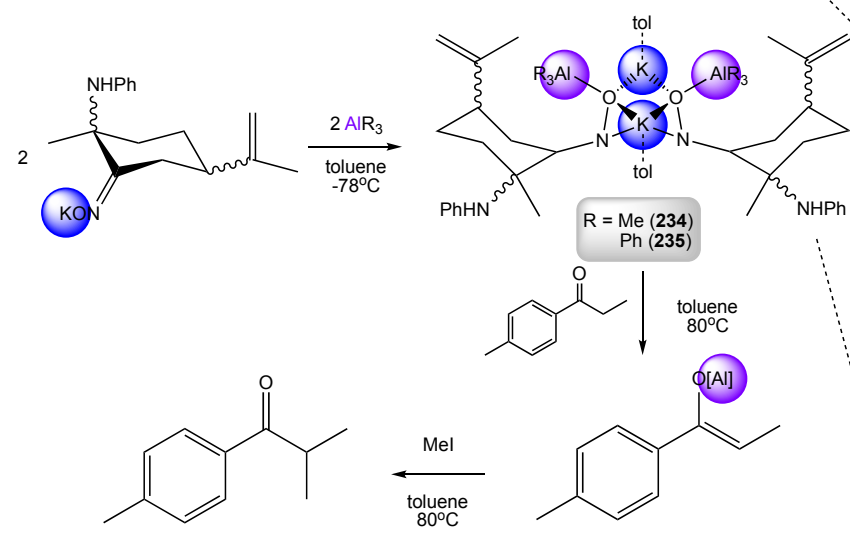

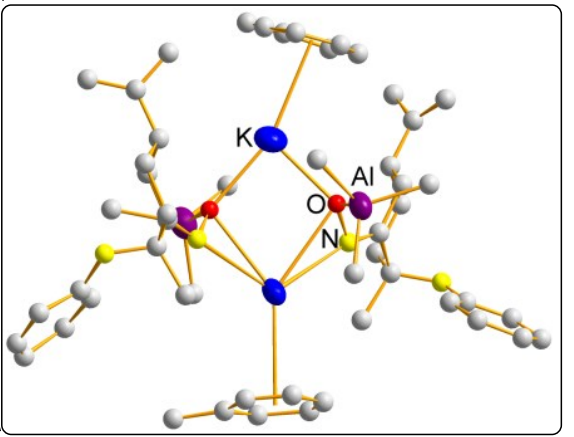

The reaction of dialumane complex LAI-AIL [236, L = dianionic $\left.\{\operatorname{DippNC}(\mathrm{Me})\}_{2}\right]$ proceeds with alkenes in a different manner depending upon whether sodium metal is present or not. ${ }^{270}$ In the absence of sodium, insertion of the alkene into the Al-Al bond was seen, yielding 237, but the reaction with conjugated alkenes such as isoprene in the presence of sodium yielded sodium aluminate 238 through [1+4] cycloaddition (scheme 142). Since the dialkene is formally reduced to a dialkane, the charge balancing presence of the sodium is necessary for the reaction to proceed.

Scheme 142. Contrasting reactions of the dialumane complex LAl-AlL [236, L = dianionic $\left.\{\text { DippNC(Me) }\}_{2}\right]$ in the absence (LHS) and presence of sodium (RHS).

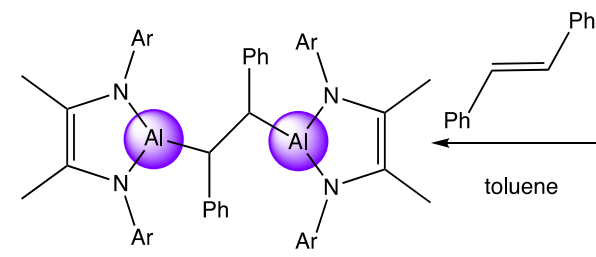

(237)

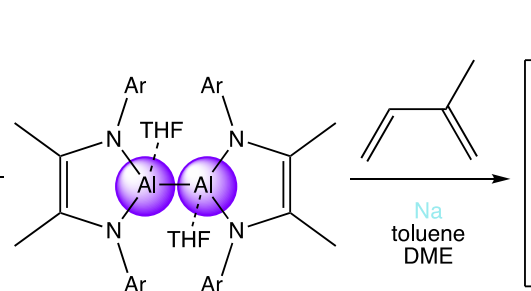

(236)

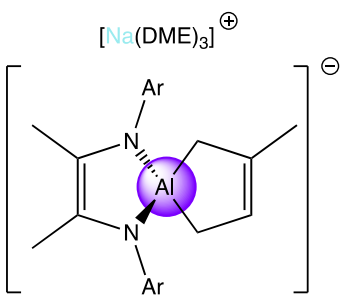

(238)

\subsubsection{Looking to the Future}


It is clear that the cooperativity between alkali-metals and group 13 metals is already well established for a variety of chemical transformations and the future appears bright for the expansion of the chemistry and synergic/synergistic properties of this combination. The high natural abundance (low cost) and low toxicity of aluminum makes it particularly attractive and it is already garnering a lot of attention in the field of catalysis, typically dominated by the redox active transition metals which are often toxic and also expensive due to their low natural abundance. Two areas in particular which look primed for future development are the recent introduction of the boryl anion 271 into alkali-metal aluminate complexes ${ }^{272}$ and the development of a potassium aluminum(I) complex $[\mathrm{KAI}(\mathrm{NON})]_{2}(239, \mathrm{NON}=$ tridentate, dianionic 4,5bis-(2,6-diisopropylanilido)-2,7-di-tert-butyl-9,9-dimethylxanthene) which can activate $\mathrm{H}_{2}$ and benzene (figure 34). ${ }^{273}$ Nucleophilic boryl species are highly reactive but this reactivity may well prove to be moderated by involvement in a bimetallic framework, providing opportunities for this reactivity to be exploited in a synergic manner while the stabilization of low-valent aluminum within a bimetallic framework might provide interesting opportunities within catalysis if facile access to a 2-electron $[\mathrm{Al}(\mathrm{I}) / \mathrm{Al}(\mathrm{III})]$ redox couple can be harnessed. 

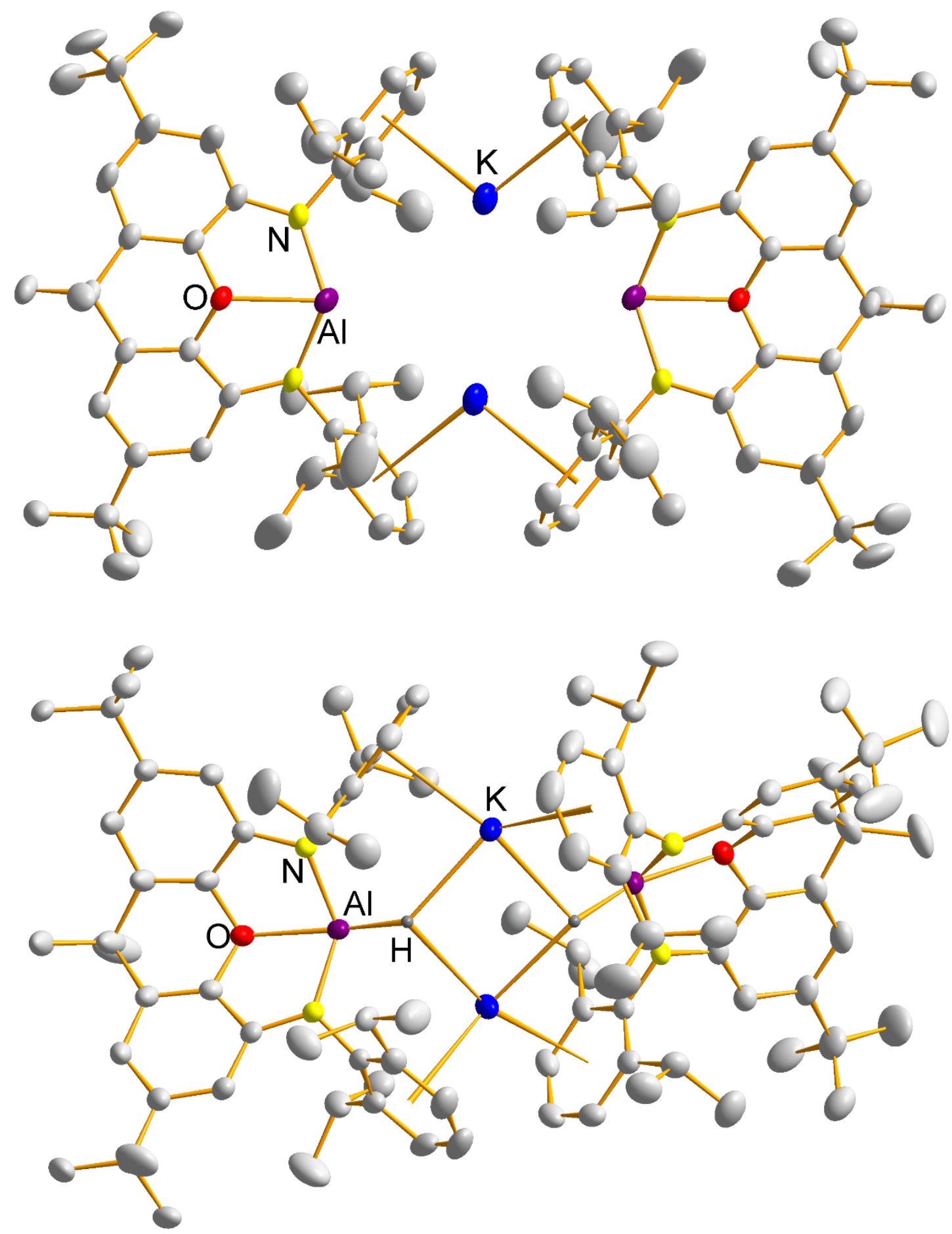

Figure 34. Top: the potassium aluminum(I) complex $[\mathrm{KAI}(\mathrm{NON})]_{2}(239)$ and bottom: the complex, $\left[K\left\{\mathrm{H}_{2} \mathrm{Al}(\mathrm{NON})\right\}\right]_{2}(\mathbf{2 4 0})$, formed when $\mathbf{2 3 9}$ is used in the activation of $\mathrm{H}_{2}$.

\section{Conclusion and Perspectives}


Alkali metal organic derivatives, especially organolithium compounds, have a long and rich history of synthetic utility in organic and organometallic chemistry. This review highlights the fact that in recent years the contribution of alkali metal chemistry to synthesis has grown even larger through the study of bimetallic compounds where the alkali metal can seemingly coerce a second metal to engage in new reaction chemistry that is outside the scope of its normal reactivity. We view this synergistic behavior as alkali metal mediation. This phenomenon is most developed within Knochel's showroom of turbo reagents, where, for example, organomagnesium compounds and organozinc compounds which are poor Brønsted bases on their own can be supplied with extra basicity ("supercharged") by alkali metal mediation, enabling them to deprotonate a wide range of aromatic and heteroaromatic $\mathrm{C}-\mathrm{H}$ bonds which are inert to these organometallic reagents in the absence of an alkali metal. In addition to this enhanced reactivity, superior selectivity and better functional group tolerance are often characteristic benefits of synergistic partnerships. In well-defined cases, bimetallic complexes of special structural design can create new regioselectivities, opening up new sites for onward functionalization, as for example witnessed in template metalation where mixed sodium-magnesium macrocyclic structures can override directed ortho metalation to access meta-meta' twofold deprotonation of aromatic substrates.

We hope this article stimulates more recognition of, and much more activity in, synergistic effects within main group bimetallic partnerships. Those examples presented here can be considered as mere demonstration models in an area suitable for massive expansion. Hitherto, the emphasis has been on stoichiometric synthesis, but looking ahead the most significant breakthroughs are likely to come in 
heterobimetallic catalysis. Future studies should also be aimed at isolating and characterizing more bimetallic intermediates prior to their utilization in onward reactions, and providing more detailed mechanistic and kinetic knowledge, supported by theoretical calculations, since understanding at present comes mainly from empirical observations.

\section{AUTHOR INFORMATION}

\section{Corresponding Author}

*E-mail: r.e.mulvey@strath.ac.uk

\section{ORCID}

Stuart Robertson 0000-0002-9330-8770

Marina Uzelac 0000-0002-5060-7017

Robert Mulvey 0000-0002-1015-2564

\section{Notes}

The authors declare no competing financial interest.

\section{Biographies}

Stuart Robertson was awarded his MChem (2002) and PhD (2006) at the University of

St. Andrews, the latter in the group of D. Woollins. This was followed by a postdoctoral position at the University of Calgary under T. Chivers before he returned to Scotland to join the Mulvey group (2009) at the University of Strathclyde. In 2012, he commenced an independent research career at Strathclyde via a Royal Society of 
Edinburgh BP Trust Fellowship and is currently a Chancellor's Fellow at Strathclyde. His research interests lie in the practical applications of bimetallic main group complexes, for example in energy storage.

Marina Uzelac obtained her BSc and MSci in Chemistry from the University of Zagreb, Croatia. She then moved to the UK, to carry out her PhD studies at the University of Strathclyde under the supervision of E. Hevia developing new gallium-mediated transformations for $\mathrm{C}-\mathrm{H}$ functionalisation and small molecule activation processes. Graduating in 2016 she then took up a postdoctoral position working in a collaborative project with the Mulvey and Hevia groups exploiting multicomponent s-block metal reagents in deprotonative metalation and C-C bond forming processes. In 2018 Marina joined the group of M. Ingleson developing new organozinc catalysts with application in bond forming processes.

Robert Emmet Mulvey gained his BSc and PhD (R. Snaith) at the University of Strathclyde (1977-1984), then spent two years as a research associate at the University of Durham (K. Wade). Returning to Strathclyde in 1986 as a Royal Society Research Fellow, he was promoted to Professor in 1995. His research spans alkali, alkali-earth and early p-block metals and exploits synergistic effects in bimetallic systems. He has received many distinguished prizes including the RSC Meldola Medal, RSC Main Group Element Award, Royal Society Wolfson Research Merit Award, Royal Society Leverhulme Trust Senior Research Fellowship, GDCh Arfvedson Schlenk Prize, and a Humboldt Research Award. A Fellow of both the Royal Society of Edinburgh and Royal Society of Chemistry, he has published approaching 300 papers.

\section{ACKNOWLEDGEMENTS}


The authors are grateful to our fellow aficionados of synergistic effects and our friends, Eva Hevia and Charles O'Hara for their invaluable advice during the compilation and writing of this review. REM also thanks the Alexander von Humboldt foundation for a Humboldt Research Award.

\section{ABBREVIATIONS}

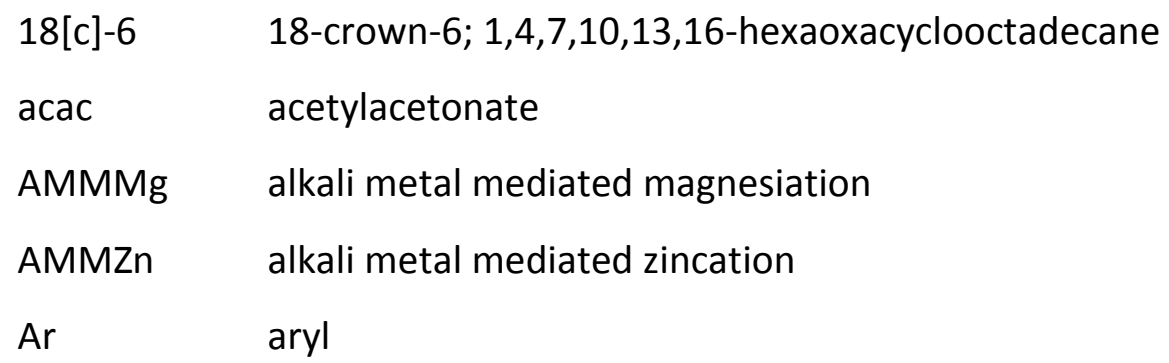




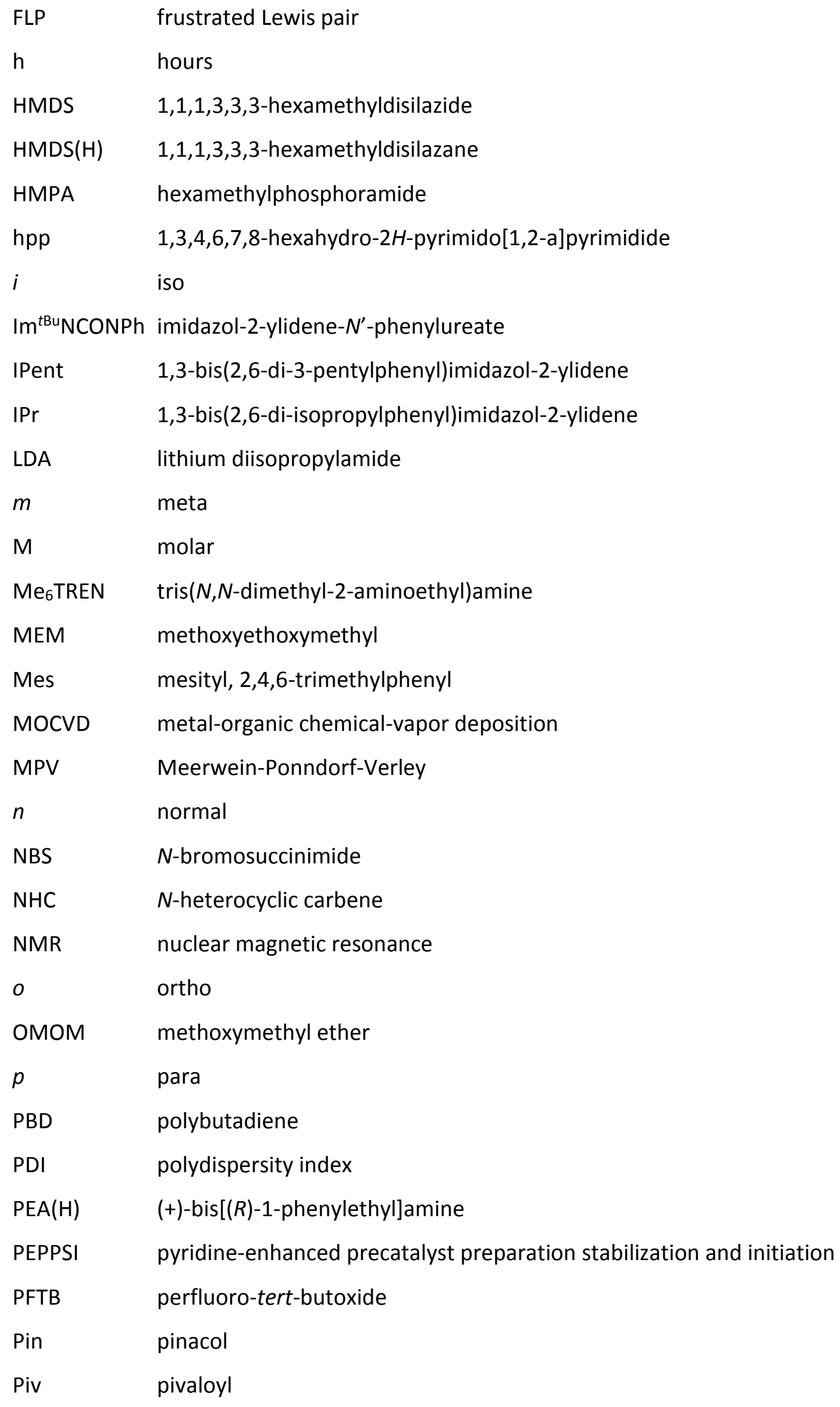




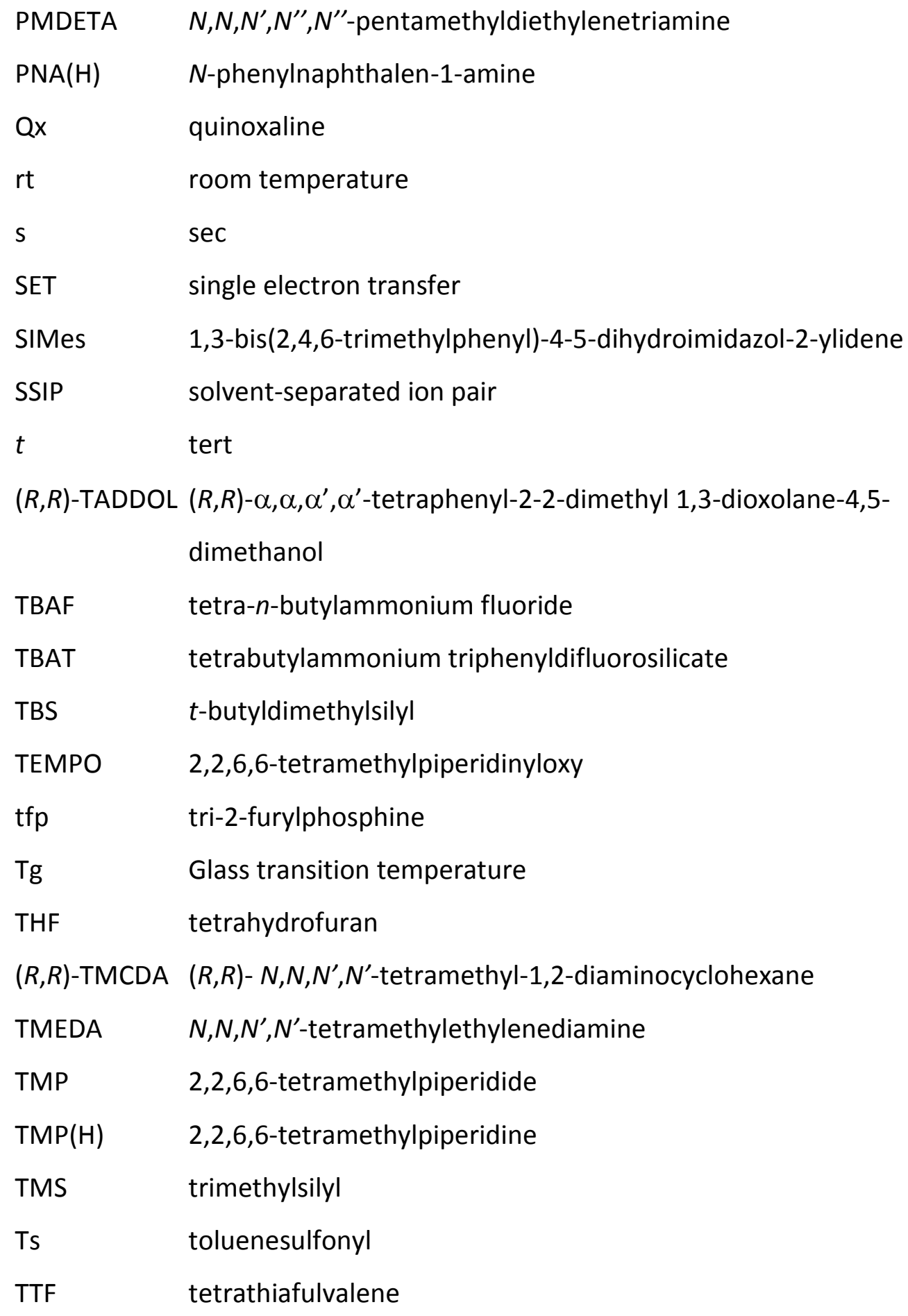

\section{REFERENCES}

(1) Wanklyn, J. A. Ueber einige neue Aethylverbindungen, welche Alkalimetalle enthalten. Liebigs Ann. 1858, 108, 67-79.

(2) Wanklyn, J. A. On some new Ethyl-Compounds containing the Alkalimetals. Proc. R. Soc. Lond. 1859, 9, 341-345. 
(3) Harrison-Marchand, A.; Mongin, F. Mixed AggregAte (MAA): A Single Concept for All Dipolar Organometallic Aggregates. 1. Structural Data. Chem. Rev. 2013, 113, 7470-7562.

(4) Mongin, F.; Harrison-Marchand, A. Mixed AggregAte (MAA): A Single Concept for All Dipolar Organometallic Aggregates. 2. Syntheses and Reactivities of Homo/HeteroMAAs. Chem. Rev. 2013, 113, 7563-7727.

(5) Schlosser, M. Zur Aktivierung Lithiumorganischer Reagenzien. J. Organomet. Chem. 1967, 8, 9-16.

(6) Lochmann, L.; Pospišil, J.; Lim, D. On the Interaction of Organolithium Compounds with Sodium and Potassium Alkoxides. A new Method for the Synthesis of Organosodium and Organopotassium Compounds. Tetrahedron Lett. 1966, 7, 257-262.

(7) Morton, A. A.; Holden, M. E. T. Polymerization VII. The Structure of the Alfin Catalyst. J. Am. Chem.Soc. 1947, 69, 1675-1681.

(8) Schlosser, M.; Simig, G. 8-Methoxyisoquinoline Derivatives through orthoSelective Metalation of 2-(3-methoxyphenyl)ethylamine. Tetrahedron Lett. 1991, 32, 1965-1966.

(9) Leroux, F.; Schlosser, M.; Zohar, E.; Marek, I. The Chemistry of Organolithium Compounds; John Wiley \& Sons Ltd.: Chichester, UK, 2004.

(10) Lochmann, L.; Janata, M. 50 Years of Superbases made from Organolithium Compounds and Heavier Alkali Metal Alkoxides. Cent. Eur. J. Chem. 2014, 12, 537-548.

(11) Ghigo, G.; Tonachini, G.; Venturello, P. Ab Initio Theoretical Investigation on the Reactivity as Bases of Mixed LiMe/KoMe Complexes. A Model for Schlosser LICKOR Superbase Tetrahedron 1996, 52, 7053-7062.

(12) Kennedy, A. R.; MacLellan, J. G.; Mulvey, R. E. Landmark Crystal Structure of an Experimentally Utilized Tetralithium-Tetrapotassium Amide-Alkoxide Superbase. Angew. Chem. Int. Ed. 2001, 40, 3245-3247.

(13) Unkelbach, C.; O'Shea, D. F.; Strohmann, C. Insights into the Metalation of Benzene and Toluene by Schlosser's Base: A Superbasic Cluster Comprising PhK, PhLi and tBuOLi. Angew. Chem. Int. Ed. 2014, 53, 553-556. 
(14) Benrath, P.; Kaiser, M.; Limbach, T.; Mondeshki, M.; Klett, J. Combining Neopentyllithium with Potassium tert-Butoxide: Formation of an AlkaneSoluble Lochmann-Schlosser Superbase. Angew. Chem. Int. Ed. 2016, 55, 10886-10889.

(15) Clegg, W.; Drummond, A. M.; Liddle, S. T.; Mulvey, R. E.; Robertson, A. A Novel Heterometallic Alkoxide: Lithium-Potassium tert-Butoxide [(Bu$\left.\left.{ }^{\mathrm{t} O}\right)_{8} \mathrm{Li}_{4} \mathrm{~K}_{4}\right]$. Chem. Commun. 1999, 1569-1570.

(16) Armstrong, D. R.; Clegg, W.; Drummond, A. M.; Liddle, S. T.; Mulvey, R. E. A Remarkable Isostructural Homologous Series of Mixed Lithium-Heavier AlkaliMetal tert-Butoxides $\left[(t-B u O)_{8} \mathrm{Li}_{4} \mathrm{M}_{4}\right](\mathrm{M}=\mathrm{Na}, \mathrm{K}, \mathrm{Rb}, \mathrm{Cs})$. J. Am. Chem.Soc. 2000, 122, 11117-11124.

(17) Aitken, R. A.; Harper, A. D.; Slawin, A. M. Z. Base-Induced Cyclisation of orthoSubstituted 2-Phenyloxazolines to Give 3-Aminobenzofurans and Related Heterocycles. Synlett 2017, 28, 1738-1742.

(18) Parisotto, S.; Boggio, P.; Prandi, C.; Venturello, P.; Deagostino, A. LIC-KOR Promoted Nitrone Reactivity: Stereoselective Synthesis of Highly Conjugated Imines and Secondary Amines. Tetrahedron Lett. 2015, 56, 5791-5794.

(19) de la Campa, R.; Garcia, D.; Rodriguez, S.; Carriedo, G. A.; Presa Soto, A. Direct Functionalization of Poly(spirophosphazene)s via the Regioselective Lithiation of the Aromatic Rings Using a Cooperative Superbase. Macromol. Rapid Commun. 2017, 38, 1700039.

(20) Fleming, P.; O'Shea, D. F. Controlled Anion Migrations with a Mixed Metal Li/K-TMP Amide: General Application to Benzylic Metalations. J. Am. Chem.Soc. 2011, 133, 1698-1701.

(21) Das, M.; O'Shea, D. F. Synthesis and Application of Benzyl-TMS Derivatives as Bench Stable Benzyl Anion Equivalents. Tetrahedron 2013, 69, 6448-6460.

(22) Das, M.; Manvar, A.; Jacolot, M.; Blangetti, M.; Jones, R. C.; O’Shea, D. F. Stereoselective Peterson Olefinations from Bench-Stable Reagents and $\mathrm{N}$ Phenyl Imines. Chem. Eur. J. 2015, 21, 8737-8740.

(23) Blangetti, M.; Müller-Bunz, H.; O’Shea, D. F. Synthesis, Separation, and Structural Analysis of Planar Chiral Carboxy-Substituted [2.2]Metacyclophanes. Tetrahedron 2013, 69, 4285-4291. 
(24) Blangetti, M.; Müller-Bunz, H.; O’Shea, D. F. First Asymmetric Synthesis of Planar Chiral [2.2]Metacyclophanes. Chem. Commun. 2013, 49, 6125-6127.

(25) Manvar, A.; Fleming, P.; O'Shea, D. F. General Ambient Temperature Benzylic Metalations Using Mixed-Metal Li/K-TMP Amide. J. Org. Chem. 2015, 80, 8727-8738.

(26) Wittig, G.; Meyer, F. J.; Lange, G. Über das Verhalten von Diphenylmetallen als Komplexbildner. Ann. 1951, 571, 167-201.

(27) Wittig, G. Komplexbildung und Reaktivität in der Metallorganischen Chemie. Angew. Chem. 1958, 70, 65-71.

(28) Wittig, G.; Bickelhaupt, F. Zur Komplexstabilisierung Alkalimetall-Organischer Verbindungen in Äther, II. Chem. Ber. 1958, 91, 865-872.

(29) Wittig, G.; Benz, E. Über Anionisierungsreaktionen mit Diphenyl-LithiumNatrium. Chem. Ber. 1958, 91, 873-882.

(30) Schümann, U.; Weiss, E. Synthesis and Structure of [\{Na(tmeda) $\left.\}_{3}\right]\left[\mathrm{LiPh}_{4}\right]$, an Organometallic Complex Containing Two Different Alkali Metals. Angew. Chem. Int. Ed. 1988, 27, 584-585.

(31) Clegg, W.; Mulvey, R. E.; Snaith, R.; Toogood, G. E.; Wade, K. The Synthesis and X-Ray Structural Characterisation of the First Mixed Alkali Metal Organonitrogen Molecular Cluster $\mathrm{LiNa}_{3}\left[\mathrm{O}=\mathrm{P}\left(\mathrm{NMe}_{2}\right)_{3}\right]_{3}\left[\mathrm{~N}=\mathrm{C}\left(\mathrm{NMe}_{2}\right)_{2}\right]_{4}$. J. Chem. Soc., Chem. Commun. 1986, 1740-1742.

(32) Barr, D.; Clegg, W.; Mulvey, R. E.; Snaith, R. Synthesis and Crystal Structure of the Mixed Alkali Metal Imide $\mathrm{Li}_{4-x} \mathrm{Na}_{2+x}\left[\mathrm{~N}=\mathrm{C}(\mathrm{Ph}) \mathrm{Bu}^{t}\right]_{6}$ : Three (Metal-Nitrogen) 2 Ring Dimers in a Triple-layered Stack. J. Chem. Soc., Chem. Commun. 1989, 57-58.

(33) Gregory, K.; Schleyer, P. v. R.; Snaith, R. Structures of Organonitrogen-Lithium Compounds: Recent Patterns and Perspectives in Organolithium Chemistry. Adv. Inorg. Chem. 1991, 37, 47-142.

(34) Filatov, A. S.; Zabula, A. V.; Spisak, S. N.; Rogachev, A. Y.; Petrukhina, M. A. Clamshell Opening in the Mixed-Metal Supramolecular Aggregates Formed by Fourfold Reduced Corannulene for Maximizing Intercalated Metal Content. Angew. Chem. Int. Ed. 2014, 53, 140-145. 
(35) Zabula, A. V.; Spisak, S. N.; Filatov, A. S.; Rogachev, A. Y.; Petrukhina, M. A. Record Alkali Metal Intercalation by Highly Charged Corannulene. Acc. Chem. Res. 2018, 51, 1541-1549.

(36) Zabula, A. V.; Filatov, A. S.; Spisak, S. N.; Rogachev, A. Y.; Petrukhina, M. A. A Main Group Metal Sandwich: Five Lithium Cations Jammed Between Two Corannulene Tetraanion Decks. Science 2011, 333, 1008-1011.

(37) Filatov, A. S.; Spisak, S. N.; Zabula, A. V.; McNeely, J.; Rogachev, A. Y.; Petrukhina, M. A. Self-Assembly of Tetrareduced Corannulene with Mixed Li$\mathrm{Rb}$ Clusters: Dynamic Transformations, Unique Structures and Record ${ }^{7} \mathrm{Li}$ NMR Shifts. Chem. Sci. 2015, 6, 1959-1966.

(38) Spisak, S. N.; Rogachev, A. Y.; Zabula, A. V.; Filatov, A. S.; Clerac, R.; Petrukhina, M. A. Tuning the Separation and Coupling of Corannulene Trianion-Radicals through Sizeable Alkali Metal Belts. Chem. Sci. 2017, 8, 3137-3145.

(39) Chen, J.; Yuan, L. Bis[ $\mu-N$-(tert-butyldimethylsilyl)quinolin-8-aminato$\left.1: 2 \mathrm{~K}^{2} N^{1}, N^{8}: N^{8}\right]\left(N, N, N^{\prime}, N^{\prime}\right.$-tetramethylethane-1,2-diamine$\left.1 \mathrm{~K}^{2} \mathrm{~N}, \mathrm{~N}^{\prime}\right)$ lithiumsodium. Acta Cryst. 2012, E68, m1474.

(40) Njua, E. Y.; Otang, M. E.; Stahl, L. Group 1 Cyclopentadienide/Group 1,14tert-Butoxide Adducts: Ion Exchange and Coordination Polymer Formation. Polyhedron 2014, 70, 101-109.

(41) Kunda, U. M. R.; Yamada, M.; Kimuro, T.; Katagiri, H.; Kondo, Y.; Hamada, F. A Hetero-Alkali Metallic ( $\mathrm{Na}, \mathrm{K}$ ) Three-Dimensional Supramolecular Assembly based on $p$-sulfonatothiacalix[4]arene. RSC Adv. 2015, 5, 30140-30144.

(42) Ziemann, S.; Krieck, S.; Görls, H.; Westerhausen, M. Potassium and Mixed Lithium/Potassium Complexes of Deprotonated 1,2Bis(neopentylamino)benzene. Z. Anorg. Allg. Chem. 2015, 641, 2140-2146.

(43) Sheng, H.; Shi, J.; Feng, Y.; Wang, H.; Jiao, Y.; Sheng, H.; Zhang, Y.; Shen, Q. Remarkable Effect of Alkali Metal on Polymerization of Cyclic Esters Catalyzed by Samarium-Alkali Metal Multinuclear Alkoxide Clusters. Dalton Trans. 2012, $41,9232-9240$. 
(44) Too, P. C.; Chan, G. H.; Tnay, Y. L.; Hirao, H.; Chiba, S. Hydride Reduction by a Sodium Hydride-lodide Composite. Angew. Chem. Int. Ed. 2016, 55, 37193723.

(45) Ong, D. Y.; Tejo, C.; Xu, K.; Hirao, H.; Chiba, S. Hydrodehalogenation of Haloarenes by a Sodium Hydride-lodide Composite. Angew. Chem. Int. Ed. 2017, 56, 1840-1844.

(46) Huang, Y.; Chan, G. H.; Chiba, S. Amide-Directed C-H Sodiation by a Sodium Hydride/lodide Composite. Angew. Chem. Int. Ed. 2017, 56, 6544-6547.

(47) Kaga, A.; Hayashi, H.; Hakamata, H.; Oi, M.; Uchiyama, M.; Takita, R.; Chiba, S. Nucleophilic Amination of Methoxy Arenes Promoted by a Sodium Hydride/lodide Composite. Angew. Chem. Int. Ed. 2017, 56, 11807-11811.

(48) Tejo, C.; Pang, J. H.; Ong, D. Y.; Oi, M.; Uchiyama, M.; Takita, R.; Chiba, S. Dearylation of Arylphosphine Oxides using a Sodium Hydride-lodide Composite. Chem. Commun. 2018, 54, 1782-1785.

(49) Hong, Z.; Ong, D. Y.; Muduli, S. K.; Too, P. C.; Chan, G. H.; Tnay, Y. L.; Chiba, S.; Nishiyama, Y.; Hirao, H.; Soo, H. S. Understanding the Origins of Nucleophilic Hydride Reactivity of a Sodium Hydride-lodide Composite. Chem. Eur. J. 2016, $22,7108-7114$.

(50) Mulvey, R. E. Avant-Garde Metalating Agents: Structural Basis of Alkali-MetalMediated Metalation. Acc. Chem. Res. 2009, 42, 743-755.

(51) Mulvey, R. E. Modern Ate Chemistry: Applications of Synergic Mixed AlkaliMetal-Magnesium or -Zinc Reagents in Synthesis and Structure Building. Organometallics 2006, 25, 1060-1075.

(52) Mulvey, R. E. s-Block Metal Inverse Crowns: Synthetic and Structural Synergism in Mixed Alkali Metal-Magnesium (or Zinc) Amide Chemistry. Chem. Commun. 2001, 1049-1056.

(53) Martinez-Martinez, A. J.; Armstrong, D. R.; Conway, B.; Fleming, B. J.; Klett, J.; Kennedy, A. R.; Mulvey, R. E.; Robertson, S. D.; O'Hara, C. T. Pre-InverseCrowns: Synthetic, Structural and Reactivity Studies of Alkali Metal Magnesiates Primed for Inverse Crown Formation. Chem. Sci. 2014, 5, 771781. 
(54) Martinez-Martinez, A. J.; Kennedy, A. R.; Mulvey, R. E.; O'Hara, C. T. Directed ortho-meta' - and meta-meta' -Dimetalations: A Template Base Approach to Deprotonation. Science 2014, 346, 834-837.

(55) Beak, P.; Brown, R. A. The ortho Lithiation of Tertiary Benzamides. J. Org. Chem. 1977, 42, 1823-1824.

(56) Mortier, J.; Moyroud, J.; Bennetau, B.; Cain, P. A. The Carboxylic Acid Group as an Effective Director of Ortho-Lithiation. J. Org. Chem. 1994, 59, 40424044.

(57) Metallinos, C.; Nerdinger, S.; Snieckus, V. N-Cumyl Benzamide, Sulfonamide, and Aryl O-Carbamate Directed Metalation Groups. Mild Hydrolytic Lability for Facile Manipulation of Directed Ortho Metalation Derived Aromatics. Org. Lett. 1999, 1, 1183-1186.

(58) Kauch, M.; Hoppe, D. Efficient Two-Step Synthesis of Salicylaldehydes via Directed ortho-Lithiation of in situ N-Silylated O-Aryl N-Isopropylcarbamates. Synthesis 2006, 1575-1577.

(59) Martinez-Martinez, A. J.; Justice, S.; Fleming, B. J.; Kennedy, A. R.; Oswald, I. D. H.; O'Hara, C. T. Templated Deprotonative Metalation of Polyaryl Systems: Facile Access to Simple, Previously Inaccessible Multi-lodoarenes. Sci. Adv. 2017, 3, e1700832.

(60) Uzelac, M.; Mulvey, R. E. Trans-Metal-Trapping: Concealed Crossover Complexes En Route to Transmetallation? Chem. Eur. J. 2018, 24, 7786-7793.

(61) Clegg, W.; Henderson, K. W.; Kennedy, A. R.; Mulvey, R. E.; O’Hara, C. T.; Rowlings, R. B.; Tooke, D. M. Regioselective Tetrametalation of Ferrocene in a Single Reaction: Extension of s-Block Inverse Crown Chemistry to the d-Block. Angew. Chem. Int. Ed. 2001, 40, 3902-3905.

(62) Baillie, S. E.; Bluemke, T. D.; Clegg, W.; Kennedy, A. R.; Klett, J.; Russo, L.; de Tullio, M.; Hevia, E. Potassium-Alkyl Magnesiates: Synthesis, Structures and Mg-H Exchange Applications of Aromatic and Heterocyclic Substrates. Chem. Commun. 2014, 50, 12859-12862.

(63) Hernan-Gomez, A.; Bradley, T. D.; Kennedy, A. R.; Livingstone, Z.; Robertson, S. D.; Hevia, E. Developing Catalytic Applications of Cooperative Bimetallics: 
Competitive Hydroamination/Trimerization Reactions of Isocyanates

Catalysed by Sodium Magnesiates. Chem. Commun. 2013, 49, 8659-8661.

(64) De Tullio, M.; Hernan-Gomez, A.; Livingstone, Z.; Clegg, W.; Kennedy, A. R.; Harrington, R. W.; Antiñolo, A.; Martinez, A.; Carrillo-Hermosilla, F.; Hevia, E. Structural and Mechanistic Insights into s-Block Bimetallic Catalysis: Sodium Magnesiate-Catalyzed Guanylation of Amines. Chem. Eur. J. 2016, 22, 1764617656.

(65) Baillie, S. E.; Clegg, W.; Garcia-Alvarez, P.; Hevia, E.; Kennedy, A. R.; Klett, J.; Russo, L. Synthesis, Structural Elucidation, and Diffusion-Ordered NMR Studies of Homoleptic Alkyllithium Magnesiates: Donor-Controlled Structural Variations in Mixed-Metal Chemistry. Organometallics 2012, 31, 5131-5141.

(66) Armstrong, D. R.; Clegg, W.; Hernan-Gomez, A.; Kennedy, A. R.; Livingstone, Z.; Robertson, S. D.; Russo, L.; Hevia, E. Probing the Metallating Ability of a Polybasic Sodium Alkylmagnesiate Supported by a Bulky Bis(Amido) Ligand: Deprotomagnesiation Reactions of Nitrogen-Based Aromatic Substrates. Dalton Trans. 2014, 43, 4361-4369.

(67) Livingstone, Z.; Hernan-Gomez, A.; Baillie, S. E.; Armstrong, D. R.; Carrella, L. M.; Clegg, W.; Harrington, R. W.; Kennedy, A. R.; Rentschler, E.; Hevia, E. Assessing the Reactivity of Sodium Alkyl-Magnesiates Towards Quinoxaline: Single Electron Transfer (SET) vs. Nucleophilic Alkylation Processes. Dalton Trans. 2016, 45, 6175-6182.

(68) Stevens, M. A.; Blair, V. L. Atom Efficient Magnesiation of N-Substituted Alkyl Indoles with a Mixed Sodium Magnesium Base. Eur. J. Inorg. Chem. 2018, 7479.

(69) Wei, J.; Liu, L.; Zhan, M.; Xu, L.; Zhang, W.-X.; Xi, Z. Magnesiacyclopentadienes as Alkaline-Earth Metallacyclopentadienes: Facile Synthesis, Structural Characterization, and Synthetic Application. Angew. Chem. Int. Ed. 2014, 53, 5634-5638.

(70) Zhu, M.; Liu, L.; Zhang, Y.; Yu, H.-T.; Zhang, W.-X.; Xi, Z. Selective Transformation of Well-Defined Alkenyllithiums to Alkenylmagnesiums via Transmetalation. Chem. Eur. J. 2018, 24, 3186-3191. 
(71) Wei, B.; Liu, L.; Zhang, W.-X.; Xi, Z. Synthesis and Structural Characterization of Butadienylcalcium-based Heavy Grignard Reagents and a $\mathrm{Ca}_{4}[\mathrm{O}]$ Inverse Crown Ether Complex. Angew. Chem. Int. Ed. 2017, 56 (9188-9192).

(72) Mulvey, R. E.; Blair, V. L.; Clegg, W.; Kennedy, A. R.; Klett, J.; Russo, L. Cleave and Capture Chemistry Illustrated through Bimetallic-Induced Fragmentation of Tetrahydrofuran. Nature Chem. 2010, 2, 588-591.

(73) Liptrot, D. J.; Hill, M. S.; Mahon, M. F. Heterobimetallic s-Block Hydrides by $\sigma$ Bond Metathesis. Chem. Eur. J. 2014, 20, 9871-9874.

(74) Gallagher, D. J.; Henderson, K. W.; Kennedy, A. R.; O'Hara, C. T.; Mulvey, R. E.; Rowlings, R. B. Hydride Encapsulation in s-Block Metal Inverse Crown Chemistry. Chem. Commun. 2002, 376-377.

(75) Ma, M.; Shen, X.; Wang, W.; Li, J.; Yao, W.; Zhu, L. Syntheses of Sterically Bulky Schiff-Base Magnesium Complexes and Their Application in the Hydrosilylation of Ketones. Eur. J. Inorg. Chem. 2016, 5057-5062.

(76) Zaragoza-Calero, S.; Francos, J.; Kennedy, A. R.; O'Hara, C. T. Solid State and Solution Studies of Lithium Tris(n-butyl)magnesiates Stabilised by Lewis Donors. Dalton Trans. 2015, 44, 7258-7267.

(77) Ortu, F.; Moxey, G. J.; Blake, A. J.; Lewis, W.; Kays, D. L. Alkaline Earth Complexes of Silylated Aminopyridinato Ligands: Homoleptic Compounds and Heterobimetallic Coordination Polymers. Inorg. Chem. 2013, 52, 1242912439.

(78) Fleming, B. J.; Garcia-Alvarez, P.; Keating, E.; Kennedy, A. R.; O’Hara, C. T. Synthesis and Structural Elucidation of a Rare Example of a tris(amido) Potassium Magnesiate. Inorg. Chim. Acta 2012, 384, 154-157.

(79) Francos, J.; Fleming, B. J.; Garcia-Alvarez, P.; Kennedy, A. R.; Reilly, K.; Robertson, G. M.; Robertson, S. D.; O'Hara, C. T. Complexity in Seemingly Simple Sodium Magnesiate Systems. Dalton Trans. 2014, 43, 14424-14431.

(80) Sarazin, Y.; Coles, S. J.; Hughes, D. L.; Hursthouse, M. B.; Bochmann, M. Cationic Brønsted Acids for the Preparation of Sn ${ }^{\text {IV }}$ Salts: Synthesis and Characterisation of $\left[\mathrm{Ph}_{3} \mathrm{Sn}\left(\mathrm{OEt}_{2}\right)\right]\left[\mathrm{H}_{2} \mathrm{~N}\left\{\mathrm{~B}\left(\mathrm{C}_{6} \mathrm{~F}_{5}\right)_{3}\right\}_{2}\right]$, $\left[\mathrm{Sn}\left(\mathrm{NMe}_{2}\right)_{3}\left(\mathrm{HNMe}_{2}\right)_{2}\right]\left[\mathrm{B}\left(\mathrm{C}_{6} \mathrm{~F}_{5}\right)_{4}\right]$ and $\left[\mathrm{Me}_{3} \mathrm{Sn}\left(\mathrm{HNMe}_{2}\right)_{2}\right]\left[\mathrm{B}\left(\mathrm{C}_{6} \mathrm{~F}_{5}\right)_{4}\right]$. Eur. J. Inorg. Chem. 2006, 3211-3220. 
(81) Graham, D. V.; Hevia, E.; Kennedy, A. R.; Mulvey, R. E. Lithium Dimethyl(amido)zinc Complexes: Contrasting Zincate (Amido = TMP) and Inverse Zincate $($ Amido $=$ HMDS) Structures on Addition of TMEDA.

Organometallics 2006, 25, 3297-3300.

(82) Naktode, K.; Bhattacharjee, J.; Nayek, H. P.; Panda, T. K. Imidazol-2-ylidene$\mathrm{N}^{\prime}$-phenylureate Ligands in Alkali and Alkaline Earth Metal Coordination Spheres - Heterocubane Core to Polymeric Structural Motif Formation. Dalton Trans. 2015, 44, 7458-7469.

(83) Glock, C.; Younis, F. M.; Ziemann, S.; Görls, H.; Imhof, W.; Krieck, S.; Westerhausen, M. 2,6-Diisopropylphenylamides of Potassium and Calcium: A Primary Amido Ligand in s-Block Metal Chemistry with an Unprecedented Catalytic Reactivity. Organometallics 2013, 32, 2649-2660.

(84) Härling, S. M.; Krieck, S.; Görls, H.; Westerhausen, M. Influence of 18-Crown6 Ether Coordination on the Catalytic Activity of Potassium and Calcium Diarylphosphinites in Hydrophosphorylation Reactions. Inorg. Chem. 2017, $56,9255-9263$.

(85) Lichtenberg, C.; Spaniol, T. P.; Peckermann, I.; Hanusa, T. P.; Okuda, J. Cationic, Neutral, and Anionic Allyl Magnesium Compounds: Unprecedented Ligand Conformations and Reactivity Toward Unsaturated Hydrocarbons. J. Am. Chem.Soc. 2013, 135, 811-821.

(86) Gallegos, C.; Tabernero, V.; Garcia-Valle, M.; Mosquera, M. E. G.; Cuenca, T.; Cano, J. Synthesis and Structure of Homo- and Heterometallic LithiumMagnesium Complexes and Their Reactivity in the ROP of rac-Lactide. Organometallics 2013, 32, 6624-6627.

(87) Catel, D.; Chevallier, F.; Mongin, F.; Gros, P. C. Chiral Organomagnesiates as Dual Reagents fro Bromine-Magnesium Exchange of 2-Bromopyridine and Access to Chiral $\alpha$-Substituted 2-Pyridylcarbinols. Eur. J. Org. Chem. 2012, 5357.

(88) Payen, O.; Chevallier, F.; Mongin, F.; Gros, P. C. Straightforward Access to Enantioenriched Pyrazyl Alcohols using Chiral Organomagnesiates.

Tetrahedron: Asymmetry 2012, 23, 1678-1682. 
(89) Catel, D.; Payen, O.; Chevallier, F.; Mongin, F.; Gros, P. C. Pyridylmagnesiates: Generation by Bromine-Metal Exchange and Enantioselective Addition to Aldehydes. Tetrahedron 2012, 68, 4018-4028.

(90) Francos, J.; Gros, P. C.; Kennedy, A. R.; O’Hara, C. T. Structural Studies of (rac)-BIPHEN Organomagnesiates and Intermediates in the Halogen-Metal Exchange of 2-Bromopyridine. Organometallics 2015, 34, 2550-2557.

(91) Hua, Y.; Guo, Z.; Han, H.; Wei, X. N,N,O-Tridentate Mixed Lithium-Magnesium and Lithium-Aluminum Complexes: Synthesis, Characterization and Catalytic Activities. Organometallics 2017, 36, 877-883.

(92) Berger, R. J. F.; Jana, S.; Fröhlich, R.; Mitzel, N. W. A Diethylhydroxylaminate Based Mixed Lithium/Beryllium Aggregate. Z. Nat. B 2015, 70, 279-282.

(93) Turner, Z. R.; Buffet, J.-C. Group 1 and 2 Cyclic (alkyl)(amino)carbene Complexes. Dalton Trans. 2015, 44, 12985-12989.

(94) Harder, S.; Ruspic, C. Probing the Salt-Metathesis Route to Bis(aryl)calcium Compounds: Structure of an Arylcalcate Complex. Eur. J. Inorg. Chem. 2015, 5743-5750.

(95) Fuentes, M. A.; Zabala, A.; Kennedy, A. R.; Mulvey, R. E. Structural Diversity in Alkali Metal and Alkali Metal Magnesiate Chemistry of the Bulky 2,6Diisopropyl-N-(trimethylsilyl)anilino Ligand. Chem. Eur. J. 2016, 22, 1496814978.

(96) Buchanan, W. D.; Ruhlandt-Senge, K. M-F Interactions and Heterobimetallics: Furthering the Understanding of Heterobimetallic Stabilization. Chem. Eur. J. 2013, 19, 10708-10715.

(97) Arrowsmith, M.; Crimmin, M. R.; Hill, M. S.; Lomas, S. L.; Heng, M. S.; Hitchcock, P. B.; Kociok-Köhn, G. Catalytic Hydroacetylenation of Carbodiimides with Homoleptic Alkaline Earth Hexamethyldisilazides. Dalton Trans. 2014, 43, 14249-14256.

(98) Knochel, P.; Krasovskiy, A.; Krasovskaya, V.; Rohbogner, C. J.; Clososki, G. C., Preparation and Use of Magnesium Amides, EP2360161, 2016

(99) Haag, B.; Mosrin, M.; Ila, H.; Malakhov, V.; Knochel, P. Regio- and Chemoselective Metalation of Arenes and Heteroarenes Using Hindered Metal Amide Bases. Angew. Chem. Int. Ed. 2011, 50, 9794-9824. 
(100) Benischke, A. D.; Ellwart, M.; Becker, M. R.; Knochel, P. Polyfunctional Zinc and Magnesium Organometallics for Organic Synthesis: Some Perspectives. Synthesis 2016, 48, 1101-1107.

(101) Ziegler, D. S.; Wei, B.; Knochel, P. Improving the Halogen-Magnesium Exchange by using new Turbo-Grignard Reagents. Chem. Eur. J. 2019, 25, 2695-2703.

(102) Krasovskiy, A.; Krasovskaya, V.; Knochel, P. Mixed Mg/Li Amides of the Type $\mathrm{R}_{2} \mathrm{NMgCl} \cdot \mathrm{LiCl}$ as Highly Efficient Bases for the Regioselective Generation of Functionalized Aryl and Heteroaryl Magnesium Compounds. Angew. Chem. Int. Ed. 2006, 45, 2958-2961.

(103) Garcia-Alvarez, P.; Graham, D. V.; Hevia, E.; Kennedy, A. R.; Klett, J.; Mulvey, R. E.; O'Hara, C. T.; Weatherstone, S. Unmasking Representative Structures of TMP-Active Hauser and Turbo-Hauser Bases. Angew. Chem. Int. Ed. 2008, 47, 8079-8081.

(104) Lin, W.; Baron, O.; Knochel, P. Highly Functionalized Benzene Syntheses by Directed Mono or Multiple Magnesiations with TMPMgCl-LiCl. Org. Lett. 2006, 8, 5673-5676.

(105) Clarke, A. J.; McNamara, S.; Meth-Cohn, O. Novel Aspects of the Metalation of Heterocycles. Side-Chain Metalation of Thiophen and Ring Metalation of Six-Membered Nitrogen Heterocycles. Tetrahedron Lett. 1974, 15, $2373-$ 2376.

(106) Mosrin, M.; Knochel, P. Regio- and Chemoselective Multiple Functionalization of Pyrimidine Derivatives by Selective Magnesiations using TMPMgCl$\cdot \mathrm{LiCl}$. Org. Lett. 2008, 10, 2497-2500.

(107) Mosrin, M.; Petrera, M.; Knochel, P. Multiple Regio- and Chemoselective Functionalizations of Pyrimidine Derivatives Using TMPMgCl$\cdot \mathrm{LiCl}$ and $\mathrm{TMP}_{2} \mathrm{Mg} \cdot 2 \mathrm{LiCl}$. Synthesis 2008, 3697-3702.

(108) Clososki, G. C.; Rohbogner, C. J.; Knochel, P. Direct Magnesiation of Polyfunctionalized Arenes and Heteroarenes Using (tmp) ${ }_{2} \mathrm{Mg} \cdot 2 \mathrm{LiCl}$. Angew. Chem. Int. Ed. 2007, 46, 7681-7684. 
(109) Rohbogner, C. J.; Clososki, G. C.; Knochel, P. A General Method for meta and para Functionalization of Arenes Using $\mathrm{TMP}_{2} \mathrm{Mg} \cdot 2 \mathrm{LiCl}$. Angew. Chem. Int. Ed. 2008, 47, 1503-1507.

(110) Mosrin, M.; Knochel, P. TMPZnCl-LiCl: A New Active Selective Base for the Directed Zincation of Sensitive Aromatics and Heteroaromatics. Org. Lett. 2009, 11, 1837-1840.

(111) Bresser, T.; Mosrin, M.; Monzon, G.; Knochel, P. Regio- and Chemoselective Zincation of Sensitive and Moderately Activated Aromatics and Heteroaromatics Using TMPZnCl.LiCl. J. Org. Chem. 2010, 75, 4686-4695.

(112) Wunderlich, S. H.; Knochel, P. (tmp) ${ }_{2} \mathrm{Zn} \cdot 2 \mathrm{MgCl}_{2} \cdot 2 \mathrm{LiCl}$ : A Chemoselective Base for the Directed Zincation of Sensitive Arenes and Heteroarenes. Angew. Chem. Int. Ed. 2007, 46, 7685-7688.

(113) Jaric, M.; Haag, B.; Unsinn, A.; Karaghiosoff, K.; Knochel, P. Highly Selective Metalations of Pyridines and Related Heterocycles Using New Frustrated Lewis Pairs ot tmp-Zinc and tmp-Magnesium Bases with $\mathrm{BF}_{3} \cdot \mathrm{OEt}_{2}$. Angew. Chem. Int. Ed. 2010, 49, 5451-5455.

(114) Wunderlich, S. H.; Knochel, P. Aluminum Bases for the Highly Chemoselective Preparation of Aryl and Heteroaryl Aluminum Compounds. Angew. Chem. Int. Ed. 2009, 48, 1501-1504.

(115) Quinio, P.; François, C.; Cuesta, A. E.; Steib, A. K.; Achrainer, F.; Zipse, H.; Karaghiosoff, K.; Knochel, P. Preparation of Tr- and Tetrasubstituted Allenes via Regioselective Lateral Metalation of Benzylic (Trimethylsilyl)alkynes Using TMPZnCl.LiCl. Org. Lett. 2015, 17, 1010-1013.

(116) Haas, D.; Hofmayer, M. S.; Bresser, T.; Knochel, P. Zincation of 4,4Dimethyloxazoline using TMPZnCl.LiCl. A New Preparation of 2Aryloxazolines. Chem. Commun. 2015, 51, 6415-6417.

(117) Yamamoto, S.; Okujo, N.; Fujita, Y.; Saito, M.; Yoshida, T.; Shinoda, S. Structures of Two Polyamine-Containing Catecholate Siderophores from Vibrio fluvialis. J. Biochem. 1993, 113, 538-544.

(118) Haas, D.; Sustac-Roman, D.; Schwarz, S.; Knochel, P. Directed Zincation with TMPZnCl.LiCl and Further Functionalization of the Tropolone Scaffold. Org. Lett. 2016, 18, 6380-6383. 
(119) Ziegler, D. S.; Greiner, R.; Lumpe, H.; Kqiku, L.; Karaghiosoff, K.; Knochel, P. Directed Zincation or Magnesiation of the 2-Pyridone and 2,7-Naphthyridone Scaffold Using TMP Bases. Org. Lett. 2017, 19, 5760-5763.

(120) Kleir, L.; Ziegler, D. S.; Rahimoff, R.; Mosrin, M.; Knochel, P. Practical LargeScale Regioselective Zincation of Chromone Using TMPZnCl.LiCl Triggered by the Presence or Absence of $\mathrm{MgCl}_{2}$. Org. Process Res. Dev. 2017, 21, 660-663.

(121) Klier, L.; Aranzamendi, E.; Ziegler, D.; Nickel, J.; Karaghiosoff, K.; Carell, T.; Knochel, P. Lewis Acid Triggered Regioselective Magnesiation and Zincation of Uracils, Uridines, and Cytidines. Org. Lett. 2016, 18, 1068-1071.

(122) Castello-Mico, A.; Nafe, J.; Higashida, K.; Karaghiosoff, K.; Gingras, M.; Knochel, P. Selective Metalations of 1,4-Dithiins and Condensed Analogues Using TMP-Magnesium and -Zinc Bases. Org. Lett. 2017, 19, 360-363.

(123) Castello-Mico, A.; Knochel, P. Zincation and Magnesiation of Functionalized Silylated Cyanohydrins Using TMP-Bases. Synthesis 2018, 50, 155-169.

(124) Nafe, J.; Auras, F.; Karaghiosoff, K.; Bein, T.; Knochel, P. Selective Functionalization of Tetrathiafulvalene Using Mg- and Zn-TMP-Bases: Preparation of Mono-, Di-, Tri-, and Tetrasubstituted Derivatives. Org. Lett. 2015, 17, 5356-5359.

(125) Nafe, J.; Knochel, P. Selective Metalation of 1,3-Dithiole-2-thiones: An Effective Preparation of New Symmetrically and Nonsymmetrically Tetraarylated Tetrathiafulvalenes. Synthesis 2016, 48, 103-114.

(126) Balkenhohl, M.; Greiner, R.; Makarov, I. S.; Heinz, B.; Karaghiosoff, K.; Zipse, H.; Knochel, P. Zn-, Mg-, and Li-TMP Bases for the Successive Regioselective Metalations of the 1,5-Naphthyridine Scaffold (TMP $=2,2,6,6-$ Tetramethylpiperidyl). Chem. Eur. J. 2017, 23, 13046-13050.

(127) Ellwart, M.; Knochel, P. Preparation of Solid, Substituted Allylic Zinc Reagents and Their Reactions with Electrophiles. Angew. Chem. Int. Ed. 2015, 54, 10662-10665.

(128) O’Brien, C. J.; Kantchev, E. A. B.; Hadei, N.; Chass, G. A.; Lough, A.; Hopkinson, A. C.; Organ, M. G. Easily Prepared Air- and Moisture-Stable Pd-NHC (NHC = N-Heterocyclic Carbene) Complexes: A Reliable, User-Friendly, Highly Active 
Palladium Precatalyst for the Suzuki-Miyaura Reaction. Chem. Eur. J. 2006, $12,4743-4748$.

(129) Bernhardt, S.; Manolikakes, G.; Kunz, T.; Knochel, P. Preparation of Solid SaltStabilized Functionalized Organozinc Compounds and their Application to Cross-Coupling and Carbonyl Addition Reactions. Angew. Chem. Int. Ed. 2011, $50,9205-9209$.

(130) Stathakis, C. I.; Bernhardt, S.; Quint, V.; Knochel, P. Improved Air-Stable Solid Aromatic and Heterocyclic Zinc Reagents by Highly Selective Metalations for Negishi Cross-Couplings. Angew. Chem. Int. Ed. 2012, 51, 9428-9432.

(131) Stathakis, C. I.; Manolikakes, S. M.; Knochel, P. TMPZnOPiv·LiCl: A New Base for the Preparation of Air-Stable Solid Zinc Pivalates of Sensitive Aromatics and Heteroaromatics. Org. Lett. 2013, 15, 1302-1305.

(132) Chen, Y.-H.; GraßI, S.; Knochel, P. Cobalt-Catalyzed Electrophilic Amination of Aryl- and Heteroarylzinc Pivalates with $\mathrm{N}$-Hydroxylamine Benzoates. Angew. Chem. Int. Ed. 2018, 57, 1108-1111.

(133) Hernan-Gomez, A.; Herd, E.; Hevia, E.; Kennedy, A. R.; Knochel, P.; Koszinowski, K.; Manolikakes, S. M.; Mulvey, R. E.; Schnegelsberg, C. Organozinc Pivalate Reagents: Segregation, Solubility, Stabilization, and Structural Insights. Angew. Chem. Int. Ed. 2014, 53, 2706-2710.

(134) Katritzky, A. R.; Luo, Z. Remarkably Easy Oxidation of Alkylzinc Reagents in Their Reactions with Electrophiles to Produce Alkoxylated Instead of the Expected Alkylated Products. Heterocycles 2001, 55, 1467-1474.

(135) Chen, Y.-H.; Tüllmann, C. P.; Ellwart, M.; Knochel, P. Preparation of Solid Polyfunctional Alkynylzinc Pivalates with Enhanced Air and Moisture Stability for Organic Synthesis. Angew. Chem. Int. Ed. 2017, 56, 9236-9239.

(136) Chen, Y.-H.; Ellwart, M.; Toupalas, G.; Ebe, Y.; Knochel, P. Preparation and Application of Solid, Salt-Stabilized Zinc Amide Enolates with Enhanced Air and Moisture Stability. Angew. Chem. Int. Ed. 2017, 56, 4612-4616.

(137) Haraguchi, R.; Matsubara, S. Preparation of the Zinc Enolate Equivalent of Amides by Zinciomethylation of loscyanates: Catalytic Asymmetric Reformatsky-Type Reaction. Synthesis 2014, 46, 2272-2282. 
(138) Perna, F. M.; Salomone, A.; Capriati, V. In Lithium Compounds in Organic Synthesis - From Fundamentals to Applications; Luisi, R.;Capriati, V., Eds.; Wiley-VCH Verlag GmbH \& Co. KGaA: Weinheim, 2014.

(139) Gilman, H.; Langham, W.; Jacoby, A. L. Metalation as a Side Reaction in the Preparation of Organolithium Compounds. J. Am. Chem.Soc. 1939, 61, 106109.

(140) Wittig, G.; Pockels, U.; Dröge, H. Über die Austauschbarkeit von Aromatisch Gebundenem Wasserstoff gegen Lithium Mittels Phenyl-lithiums. Chem. Ber. 1938, 71, 1903-1912.

(141) Boudier, A.; Bromm, L. O.; Lotz, M.; Knochel, P. New Applications of Polyfunctional Organometallic Compounds in Organic Synthesis. Angew. Chem. Int. Ed. 2000, 39, 4414-4435.

(142) Bao, R. L.-Y.; Zhao, R.; Shi, L. Progress and Developments in the Turbo Grignard Reagent i-PrMgCl-LiCl: a Ten-Year Journey. Chem. Commun. 2015, $51,6884-6900$.

(143) Krasovskiy, A.; Knochel, P. A LiCl-Mediated Br/Mg Exchange Reaction for the Preparation of Functionalized Aryl- and Heteroarylmagnesium Compounds from Organic Bromides. Angew. Chem. Int. Ed. 2004, 43, 3333-3336.

(144) Krasovskiy, A.; Straub, B. F.; Knochel, P. Highly Efficient Reagents for Br/Mg Exchange. Angew. Chem. Int. Ed. 2006, 45, 159-162.

(145) Hauk, D.; Lang, S.; Murso, A. Minimization of Side Reactions in Bromine Magnesium Exchanges with $i-\mathrm{PrMg} / \mathrm{Cl} / \mathrm{LiCl}$ and s-BuMgCl$/ \mathrm{LiCl}$ Mixtures. Org. Process Res. Dev. 2006, 10, 733-738.

(146) Kunz, T.; Knochel, P. Synthesis of Functionalized Benzo[b]thiophenes by the Intramolecular Copper-Catalyzed Carbomagnesiation of Alkynyl(aryl)thioethers. Angew. Chem. Int. Ed. 2012, 51, 1958-1961.

(147) Nickel, J.; Fernandez, M.; Klier, L.; Knochel, P. Synthesis of Pyrrolo[2,3d]pyrimidines by Copper-Mediated Carbomagnesiations of $N$-Sulfonyl Ynamides and Application to the Preparation of Rigidin A and a 7Azaserotonin Derivative. Chem. Eur. J. 2016, 22, 14397-14400. 
(148) Liu, C.-Y.; Knochel, P. Preparation of Polyfunctional Arylmagnesium Reagents Bearing a Triazene Moiety. A New Carbazole Synthesis. Org. Lett. 2005, 7, 2543-2546.

(149) Morita, T.; Fuse, S.; Nakamura, H. Generation of an 4-Isoxazolyl Anion Species: Facile Access to Multifunctionalized Isoxazoles. Angew. Chem. Int. Ed. 2016, 55, 13580-13584.

(150) Taylor, R. D.; MacCoss, M.; Lawson, A. D. G. Rings in Drugs. J. Med. Chem. 2014, 57, 5845-5859.

(151) Blasberg, F.; Bolte, M.; Wagner, M.; Lerner, H.-W. An Approach to Pin Down the Solid-State Structure of the "Turbo Grignard". Organometallics 2012, 31, 1001-1005.

(152) Sakamoto, S.; Imamoto, T.; Yamaguchi, K. Constitution of Grignard Reagent $\mathrm{RMgCl}$ in Tetrahydrofuran. Org. Lett. 2001, 3, 1793-1795.

(153) McLaughlin, M.; Belyk, K. M.; Qian, G.; Reamer, R. A.; Chen, C.-y. Synthesis of a-Hydroxyacetophenones. J. Org. Chem. 2012, 77, 5144-5148.

(154) Sälinger, D.; Brückner, R. The First Asymmetric Halogen/Metal-Exchange Reaction: Desymmetrization of Alcohols with Enantiotopic Bromoarene Substituents. Chem. Eur. J. 2009, 15, 6688-6703.

(155) Farkas, J.; Stoudt, S. J.; Hanawalt, E. M.; Pajerski, A. D.; Richey, H. G. Reactions of Organomagnesates and Aryl Halides: Metalation and Nucleophilic Substitution. Organometallics 2004, 23, 423-427.

(156) Ziegler, D. S.; Karaghiosoff, K.; Knochel, P. Generation of Aryl and Heteroaryl Magnesium Reagents in Toluene by $\mathrm{Br} / \mathrm{Mg}$ or $\mathrm{Cl} / \mathrm{Mg}$ Exchange. Angew. Chem. Int. Ed. 2018, 57, 6701-6704.

(157) Becker, M. R.; Knochel, P. Practical Continuous-Flow Trapping Metalations of Functionalized Arenes and Heteroarenes Using TMPLi in the Presence of Mg, Zn, Cu, or La Halides. Angew. Chem. Int. Ed. 2015, 54, 12501-12505.

(158) Becker, M. R.; Ganiek, M. A.; Knochel, P. Practical and Economic Lithiations of Functionalized Arenes and Heteroarenes using $\mathrm{Cy}_{2} \mathrm{NLi}$ in the Presence of $\mathrm{Mg}$, Zn or La Halides in a Continuous Flow. Chem. Sci. 2015, 6, 6649-6653. 
(159) Ganiek, M. A.; Becker, M. R.; Ketels, M.; Knochel, P. Continuous Flow Magnesiation or Zincation of Acrylonitrilies, Acrylates, and Nitroolefins. Application to the Synthesis of Butenolides. Org. Lett. 2016, 18, 828-831.

(160) Becker, M. R.; Knochel, P. High-Temperature Continuous-Flow Zincations of Functionalized Arenes and Heteroarenes Using $\left(\mathrm{C}_{2} \mathrm{~N}\right)_{2} \mathrm{Zn} \cdot \mathrm{LiCl}$ Org. Lett. 2016, $18,1462-1465$.

(161) Ketels, M.; Konrad, D. B.; Karaghiosoff, K.; Trauner, D.; Knochel, P. Selective Lithiation, Magnesiation, and Zincation of Unsymmetrical Azobenzenes Using Continuous Flow. Org. Lett. 2017, 19, 1666-1669.

(162) Ketels, M.; Ziegler, D. S.; Knochel, P. Selective Zincation of 1,2Dicyanobenzene and Related Benzonitriles in Continuous Flow Using In Situ Trapping Metalations. Synlett 2017, 28, 2817-2822.

(163) Feng, C.; Cunningham, D. W.; Easter, Q. T.; Blum, S. A. Role of LiCl in Generating Soluble Organozinc Reagents. J. Am. Chem.Soc. 2016, 138, 1115611159.

(164) Shen, Z.-L.; Knochel, P. C60-Catalyzed Preparation of Aryl and Heteroaryl Magnesium and Zinc Reagents using Mg/LiCl. ACS Catal. 2015, 5, 2324-2328.

(165) Fullerenes: Chemistry and Reactions; Hirsch, A.; Brettreich, M., Eds.; WileyVCH Verlag GmbH \& Co. KGaA: Weinheim, 2005.

(166) Benischke, A. D.; Le Corre, G.; Knochel, P. Preparation of Polyfunctional Organozinc Halides by an InX $X_{3}$ - and LiCl-Catalyzed Zinc Insertion to Aryl and Heteroaryl lodides and Bromides. Chem. Eur. J. 2017, 23, 778-782.

(167) Shen, Z.-L.; Knochel, P. Stereoselective Preparation of Polyfunctional Alkenylindium(III) Halides and Their Cross-Coupling with Unsaturated Halides. Chem. Eur. J. 2015, 21, 7061-7065.

(168) Quinio, P.; Sustac Roman, D.; Leon, T.; William, S.; Karaghiosoff, K.; Knochel, P. Transition-Metal-Free Cross-Coupling of Aryl and $\mathrm{N}$-Heteroaryl Cyanides with Benzylic Zinc Reagents. Org. Lett. 2015, 17, 4396-4399.

(169) Balkenhohl, M.; François, C.; Sustac Roman, D.; Quinio, P.; Knochel, P. Transition-Metal-Free Amination of Pyridine-2-sulfonyl Chloride and Related $N$-Heterocycles Using Magnesium Amides. Org. Lett. 2017, 19, 536-539. 
(170) Mulvey, R. E.; Mongin, F.; Uchiyama, M.; Kondo, Y. Deprotonative Metalation Using Ate Compounds: Synergy, Synthesis, and Structure Building. Angew. Chem. Int. Ed. 2007, 46, 3802-3824.

(171) Wang, Y.; Xie, Y.; Abraham, M. Y.; Gilliard Jr., R. J.; Wei, P.; Campana, C. F.; Schaefer III, H. F.; Schleyer, P. v. R.; Robinson, G. H. NHC-Stabilized Triorganozincates: Syntheses, Structures, and Transformation to Abnormal Carbene-Zinc Complexes. Angew. Chem. Int. Ed. 2012, 51, 10173-10176.

(172) Balloch, L.; Garden, J. A.; Kennedy, A. R.; Mulvey, R. E.; Rantanen, T.; Robertson, S. D.; Snieckus, V. Dizincation of a 2-Substituted Thiophene: Constructing a Cage with a [16]Crown-4 Zincocyclic Core. Angew. Chem. Int. Ed. 2012, 51, 6934-6937.

(173) Clegg, W.; Crosbie, E.; Dale-Black, S. H.; Hevia, E.; Honeyman, G. W.; Kennedy, A. R.; Mulvey, R. E.; Ramsay, D. L.; Robertson, S. D. Structurally Defined Zincated and Aluminated Complexes of Ferrocene Made by Alkali-Metal Synergistic Syntheses. Organometallics 2015, 34, 2580-2589.

(174) Hevia, E.; Kennedy, A. R.; McCall, M. D. Assessing the Reactivity of Sodium Zincate [(TMEDA)Na(TMP)Zn ${ }^{t} \mathrm{Bu}_{2}$ ] Towards Benzoylferrocene: Deprotonative Metalation vs. Alkylation Reactions. Dalton Trans. 2012, 41, 98-103.

(175) Hevia, E.; Honeyman, G. W.; Kennedy, A. R.; Mulvey, R. E. Trapping, Stabilization, and Characterization of an Enolate Anion of a 1,6-Adduct of Benzophenone Chelated by a Sodium Alkylamidozincate Cation. J. Am. Chem.Soc. 2005, 127, 13106-13107.

(176) Mulvey, R. E. An Alternative Picture of Alkali-Metal-Mediated Metallation: Cleave and Capture Chemistry. Dalton Trans. 2013, 42, 6676-6693.

(177) Bates, R. B.; Kroposki, L. M.; Potter, D. E. Cycloreversions of Anions from Tetrahydrofurans. A Convenient Synthesis of Lithium Enolates of Aldehydes. J. Org. Chem. 1972, 37, 560-562.

(178) Kennedy, A. R.; Klett, J.; Mulvey, R. E.; Wright, D. S. Synergic Sedation of Sensitive Anions: Alkali-Mediated Zincation of Cyclic Ethers and Ethene. Science 2009, 326, 706-708.

(179) Kennedy, A. R.; Mulvey, R. E.; Ramsay, D. L.; Robertson, S. D. Heterobimetallic Metallation Studies of $\mathrm{N}, \mathrm{N}$-dimethylphenylethylamine (DMPEA): Benzylic C-H 
Bond Cleavage/Dimethylamino Capture or Intact DMPEA Complex. Dalton Trans. 2015, 44, 5875-5887.

(180) Armstrong, D. R.; Balloch, L.; Crawford, J. J.; Fleming, B. J.; Hogg, L. M.; Kennedy, A. R.; Klett, J.; Mulvey, R. E.; O’Hara, C. T.; Orr, S. A.et al. Single Electron Transfer (SET) Activity of the Dialkyl-Amido Sodium Zincate $\left[(T M E D A) \cdot \mathrm{Na}(\mu-\mathrm{TMP})\left(\mu-{ }^{\mathrm{B}} \mathrm{Bu}\right) \mathrm{Zn}\left({ }^{t} \mathrm{Bu}\right)\right]$ Towards TEMPO and Chalcone. Chem. Commun. 2012, 48, 1541-1543.

(181) Garden, J. A.; Kennedy, A. R.; Mulvey, R. E.; Robertson, S. D. Ambient Temperature Zincation of N-Boc Pyrrolidine and its Solvent Dependency. Chem. Commun. 2012, 48, 5265-5267.

(182) Francos, J.; Kennedy, A. R.; O'Hara, C. T. Synthetic and Reactivity Studies of Hetero-tri-anionic Sodium Zincates. Dalton Trans. 2016, 45, 6222-6233.

(183) Armstrong, D. R.; Clegg, W.; Dale, S. H.; Graham, D. V.; Hevia, E.; Hogg, L. M.; Honeyman, G. W.; Kennedy, A. R.; Mulvey, R. E. Dizincation and Dimagnesiation of Benzene using Alkali-Metal-Mediated Metallation. Chem. Commun. 2007, 598-600.

(184) Baillie, S. E.; Blair, V. L.; Blakemore, D. C.; Hay, D.; Kennedy, A. R.; Pryde, D. C.; Hevia, E. New Lithium-Zincate Approaches for the Selective Functionalisation of Pyrazine: Direct Dideprotozincation vs. Nucleophilic Alkylation. Chem. Commun. 2012, 48, 1985-1987.

(185) Kadiyala, R. R.; Tilly, D.; Nagaradja, E.; Roisnel, T.; Matulis, V. E.; Ivashkevich, O. A.; Halauko, Y. S.; Chevallier, F.; Gros, P. C.; Mongin, F. Computed CH Acidity of Biaryl Compounds and Their Deprotonative Metalation by Using a Mixed Lithium/Zinc-TMP Base. Chem. Eur. J. 2013, 19, 7944-7960.

(186) Amara, R.; Bentabed-Ababsa, G.; Hedidi, M.; Khoury, J.; Awad, H.; Nassar, E.; Roisnel, T.; Dorcet, V.; Chevallier, F.; Fajloun, Z.et al. Synthesis of $N$-Aryl and $N$-Heteroaryl $\gamma^{-}, \delta$-, and $\varepsilon$-Lactams Using Deprotometalation-Iodination and N-Arylation, and Properties Thereof. Synthesis 2017, 49, 4500-4516.

(187) Hedidi, M.; Erb, W.; Lassagne, F.; Halauko, Y. S.; Ivashkevich, O. A.; Matulis, V. E.; Roisnel, T.; Bentabed-Ababsa, G.; Mongin, F. Functionalization of Pyridyl Ketones using Deprotolithiation-in situ Zincation. RSC Adv. 2016, 6, 6318563189. 
(188) Marquise, N.; Bretel, G.; Lassagne, F.; Chevallier, F.; Roisnel, T.; Dorcet, V.; Halauko, Y. S.; Ivashkevich, O. A.; Matulis, V. E.; Gros, P. C.et al. DeprotoMetallation using Mixed Lithium-Zinc and Lithium-Copper Bases and Computed CH Acidity of 2-Substituted Quinolines. RSC Adv. 2014, 4, 1960219612.

(189) Chevallier, F.; Blin, T.; Nagaradja, E.; Lassagne, F.; Roisnel, T.; Halauko, Y. S.; Matulis, V. E.; Ivashkevich, O. A.; Mongin, F. Deproto-Metallation and Computed CH Acidity of 2-aryl-1,2,3-triazoles. Org. Biomol. Chem. 2012, 10, 4878-4885.

(190) Nagaradja, E.; Chevallier, F.; Roisnel, T.; Dorcet, V.; Halauko, Y. S.; Ivashkevich, O. A.; Matulis, V. E.; Mongin, F. Deproto-Metallation using a Mixed Lithium-Zinc Base and Computed CH Acidity of 1-aryl $1 \mathrm{H}-$ benzotriazoles and 1-aryl $1 H$-indazoles. Org. Biomol. Chem. 2014, 12, 14751487.

(191) Nagaradja, E.; Bentabed-Ababsa, G.; Scalabrini, M.; Chevallier, F.; Philippot, S.; Fontanay, S.; Duval, R. E.; Halauko, Y. S.; Ivashkevich, O. A.; Matulis, V. E.et al. Deprotometalation-lodolysis and Computed CH Acidity of 1,2,3- and 1,2,4Triazoles. Application to the Synthesis of Resveratrol Analogues. Bioorg. Med. Chem. 2015, 23, 6355-6363.

(192) Hedidi, M.; Bentabed-Ababsa, G.; Derdour, A.; Roisnel, T.; Dorcet, V.; Chevallier, F.; Picot, L.; Thiery, V.; Mongin, F. Synthesis of C,N'-Linked BisHeterocycles using a Deprotometalation-lodination-N-Arylation Sequence and Evaluation of Their Antiproliferative Activity in Melanoma Cells. Bioorg. Med. Chem. 2014, 22, 3498-3507.

(193) Messaoud, M. Y. A.; Bentabed-Ababsa, G.; Hedidi, M.; Derdour, A.; Chevallier, F.; Halauko, Y. S.; Ivashkevich, O. A.; Matulis, V. E.; Picot, L.; Thiery, V.et al. Deproto-Metallation of N-Arylated Pyrroles and Indoles using a Mixed Lithium-Zinc Base and Regioselectivity-Computed CH Acidity Relationship. Beilstein J. Org. Chem. 2015, 11, 1475-1485.

(194) Tazi, M.; Erb, W.; Halauko, Y. S.; Ivashkevich, O. A.; Matulis, V. E.; Roisnel, T.; Dorcet, V.; Mongin, F. From 2- to 3-Substituted Ferrocene Carboxamides or 
How to Apply Halogen "Dance" to the Ferrocene Series. Organometallics $2017,36,4770-4778$.

(195) Hedidi, M.; Maillard, J.; Erb, W.; Lassagne, F.; Halauko, Y. S.; Ivashkevich, O. A.; Matulis, V. E.; Roisnel, T.; Dorcet, V.; Hamze, M.et al. Fused Systems Based on 2-Aminopyrimidines: Synthesis Combining Deprotolithiation-in situ Zincation with N-Arylation Reactions and Biological Properties. Eur. J. Org. Chem. 2017, 5903-5915.

(196) Sreeshailam, A.; Dayaker, G.; Ramana, D. V.; Chevallier, F.; Roisnel, T.; Komagawa, S.; Takita, R.; Uchiyama, M.; Krishna, P. R.; Mongin, F. Synthesis of Both Enantiomers of Ferrocene[1,2-c]1H-quinolin-2-one by Diastereoselective Deproto-Zincation of Sugar-Derived Ferrocene Esters. RSC Adv. 2012, 2, 7030-7032.

(197) Dayaker, G.; Tilly, D.; Chevallier, F.; Hilmersson, G.; Gros, P. C.; Mongin, F. Enantioselective Metalation of $\mathrm{N}, \mathrm{N}$-Diisopropylferrocenecarboxamide and Methyl Ferrocenecarboxylate Using Lithium-Metal Chiral Bases. Eur. J. Org. Chem. 2012, 6051-6057.

(198) Armstrong, D. R.; Baillie, S. E.; Blair, V. L.; Chabloz, N. G.; Diez, J.; GarciaAlvarez, J.; Kennedy, A. R.; Robertson, S. D.; Hevia, E. Alkali-Metal-Mediated Zincation (AMMZn) Meets N-Heterocyclic Carbene (NHC) Chemistry: Zn-H Exchange Reactions and Structural Authentification of a Dinuclear Au(I) Complex with a NHC Anion. Chem. Sci. 2013, 4, 4259-4266.

(199) Waters, J. B.; Goicoechea, J. M. Alkali Metal Salts of Ditopic Carbanionic Carbenes as Reagents for the Synthesis of Novel Complexes of Group 12 and 14 Metals. Dalton Trans. 2014, 43, 14239-14248.

(200) Armstrong, D. R.; Garden, J. A.; Kennedy, A. R.; Leenhouts, S. M.; Mulvey, R. E.; O’Keefe, P.; O’Hara, C. T.; Steven, A. Evaluating cis-2,6-Dimethylpiperidide (cis-DMP) as a Base Component in Lithium-Mediated Zincation Chemistry. Chem. Eur. J. 2013, 19, 13492-13503.

(201) Hernan-Gomez, A.; Herd, E.; Uzelac, M.; Cadenbach, T.; Kennedy, A. R.; Borilovic, I.; Aromi, G.; Hevia, E. Zincate-Mediated Arylation Reactions of Acridine: Pre- and Postarylation Structural Insights. Organometallics 2015, $34,2614-2623$. 
(202) Hyodo, I.; Tobisu, M.; Chatani, N. Regioselective C-H Bond Functionalizations of Acridines using Organozinc Reagents. Chem. Commun. 2012, 48, 308-310.

(203) Guo, Z.; Wang, Y.; Cao, W.; Chao, J.; Wei, X. Structural Diversity of AlkaliMetal (Li, Na, K) Alkyl Zincates Containing Bidentate Aminopyrrolyl Ligands: From Molecular Complexes to Coordination Polymers. Dalton Trans. 2017, $46,2765-2769$.

(204) Nguyen, M. T.; Gabidullin, B.; Nikonov, G. I. Neutral and Anionic Zinc Compounds Supported by a Bis(imino)phenyl NCN Ligand. Dalton Trans. 2018, 47, 4607-4612.

(205) Armstrong, D. R.; Garden, J. A.; Kennedy, A. R.; Mulvey, R. E.; Robertson, S. D. Modifying Alkylzinc Reactivity with 2,2'-Dipyridylamide: Activation of $t \mathrm{Bu}-\mathrm{Zn}$ Bonds for para-Alkylation of Benzophenone. Angew. Chem. Int. Ed. 2013, 52, 7190-7193.

(206) Armstrong, D. R.; Emerson, H. S.; Hernan-Gomez, A.; Kennedy, A. R.; Hevia, E. New Supramolecular Assemblies in Heterobimetallic Chemistry: Synthesis of a Homologous Series of Unsolvated Alkali-Metal Zincates. Dalton Trans. 2014, $43,14229-14238$.

(207) Morisako, S.; Shang, R.; Yamamoto, Y. Synthesis of a Sterically Demanding Dispiropiperidine and Its Application in Monoamidodialkyl Zincate Complexes. Inorg. Chem. 2016, 55, 10767-10773.

(208) Armstrong, D. R.; Davidson, M. G.; Garcia-Vivo, D.; Kennedy, A. R.; Mulvey, R. E.; Robertson, S. D. Monomerizing Alkali-Metal 3,5-Dimethylbenzyl Salts with Tris(N,N-dimethyl-2-aminoethyl)amine (Me $\left.{ }_{6} T R E N\right):$ Structural and Bonding Implications. Inorg. Chem. 2013, 52, 12023-12032.

(209) Davidson, M. G.; Garcia-Vivo, D.; Kennedy, A. R.; Mulvey, R. E.; Robertson, S. D. Exploiting $\sigma / \pi$ Coordination Isomerism to Prepare Homologous Organoalkali Metal (Li, Na, K) Monomers with Identical Ligand Sets. Chem. Eur. J. 2011, 17, 3364-3369.

(210) Roberts, A. J.; Kennedy, A. R.; McLellan, R.; Robertson, S. D.; Hevia, E. Synthesis, Structure and Solution Studies on Mixed Aryl/Alkyl Lithium Zincates. Eur. J. Inorg. Chem. 2016, 4752-4760. 
(211) Clegg, W.; Forbes, G. C.; Kennedy, A. R.; Mulvey, R. E.; Liddle, S. T. PotassiumZinc Induced Synergic Enhancement of the Basicity of Hexamethyldisilazide (HMDS) towards Methylbenzene Molecules. Chem. Commun. 2003, 406-407.

(212) Liu, Y.-F.; Zhai, D.-D.; Zhang, X.-Y.; Guan, B.-T. Potassium-Zincate-Catalyzed Benzylic C-H Bond Addition of Diarylmethanes to Styrenes. Angew. Chem. Int. Ed. 2018, 57, 8245-8249.

(213) Su, Y.; Zhao, Y.; Gao, J.; Dong, Q.; Wu, B.; Yang, X.-J. Alkali Metal and Zinc Complexes of a Bridging 2,5-Diamino-1,4-Benzoquinonediimine Ligand. Inorg. Chem. 2012, 51, 5889-5896.

(214) Liu, J.; Vieille-Petit, L.; Linden, A.; Luan, X.; Dorta, R. Synthesis and Structural Characterization of $\mathrm{Li}^{\prime}, \mathrm{Zn}^{\prime \prime}, \mathrm{Cd}^{\prime \prime}$, and $\mathrm{Hg}^{\prime \prime}$ Complexes Containing a Fluorinated $\beta$-Diketiminate Ligand. J. Organomet. Chem. 2012, 719, 80-86.

(215) Rad, B. R.; Herrmann, D.; Lescop, C.; Wolf, R. A Tetradentate Metalloligand: Synthesis and Coordination Behaviour of a 2-Pyridyl-Substituted Cyclobutadiene Iron Complex. Dalton Trans. 2014, 43, 4247-4250.

(216) Zhan, M.; Zhang, S.; Zhang, W.-X.; Xi, Z. Diazo Compounds as Electrophiles To React with 1,4-Dilithio-1,3-dienes: Efficient Synthesis of 1-Imino-pyrrole Derivatives. Org. Lett. 2013, 15, 4182-4185.

(217) Pinkert, D.; Demeshko, S.; Schax, F.; Braun, B.; Meyer, F. J.; Limberg, C. A Dinuclear Molecular Iron(II) Silicate with Two High-Spin Square-Planar FeO $\mathrm{F}_{4}$ Units. Angew. Chem. Int. Ed. 2013, 52, 5155-5158.

(218) Schax, F.; Braun, B.; Limberg, C. A Tripodal Trisilanol Ligand and Its Complexation Behaviour towards Cu', Cu", and Zn". Eur. J. Inorg. Chem. 2014, 2124-2130.

(219) Sun, Y.; Wang, L.; Yu, D.; Tang, N.; Wu, J. Zinc/Magnesium-Sodium/Lithium Heterobimetallic Triphenolates: Synthesis, Characterization, and Application as Catalysts in the Ring-Opening Polymerization of L-Lactide and $\mathrm{CO}_{2} /$ Epoxide Coupling. J. Mol. Catal. A. 2014, 393, 175-181.

(220) Saßmannshausen, J.; Klett, J.; Kennedy, A. R.; Parkinson, J. A.; Armstrong, D. Taming the Shrew: [TMEDALi- $\mathrm{Zn}\left(\mathrm{C}_{2} \mathrm{H}_{4}\right)_{2} \mathrm{~L}$ ] as a Model Compound for Anionic Ethene Polymerisation. New J. Chem. 2013, 37, 494-501. 
(221) Li, H.; Zhang, L.; Wang, Z.-X.; Hu, Y. Ethylene Polymerization Initiated by Tertiary Diamine/n-Butyllithium Complexes: An Interpretation from Density Functional Theory Study. J. Phys. Chem. A 2010, 114, 2697-2700.

(222) Coles, M. P.; Hitchcock, P. B. Bicyclic Guanidinates in Mono- and Di-Valent Metal Complexes, Including Group 1/2 and Group 1/12 Heterometallic Systems. Aust. J. Chem. 2013, 66, 1124-1130.

(223) Lichtenberg, C.; Spaniol, T. P.; Perrin, L.; Maron, L.; Okuda, J. Reversible 1,4Insertion of Pyridine Into a Highly Polar Metal-Carbon Bond: Effect of the Second Metal. Chem. Eur. J. 2012, 18, 6448-6452.

(224) Miller, L. Z.; Shatruk, M.; McQuade, D. T. Alkali Metal Oxides Trapped by Diethylzinc. Chem. Commun. 2014, 50, 8937-8940.

(225) Finholt, A. E.; Bond Jr., A. C.; Schlesinger, H. I. Lithium Aluminium Hydride, Aluminium Hydride and Lithium Gallium Hydride, and Some of their Applications in Organic and Inorganic Chemistry. J. Am. Chem.Soc. 1947, 69, 1199-1203.

(226) Nystrom, R. F.; Brown, W. G. Reduction of Organic Compounds by Lithium Aluminum Hydride. I. Aldehydes, Ketones, Esters, Acid Chlorides and Acid Anhydrides. J. Am. Chem.Soc. 1947, 69, 1197-1199.

(227) Nystrom, R. F.; Brown, W. G. Reduction of Organic Compounds by Lithium Aluminum Hydride. II. Carboxylic Acids. J. Am. Chem.Soc. 1947, 69, 25482549.

(228) Nystrom, R. F.; Brown, W. G. Reduction of Organic Compounds by Lithium Aluminum Hydride. III. Halides, Quinones, Miscellaneous Nitrogen Compounds. J. Am. Chem.Soc. 1948, 70, 3738-3740.

(229) Lansbury, P. T.; Peterson, J. O. Lithium Tetrakis-(N-dihydropyridyl)-aluminate: Structure and Reducing Properties. J. Am. Chem.Soc. 1963, 85, 2236-2242.

(230) Sakintuna, B.; Lamari-Darkrim, F.; Hirscher, M. Metal Hydride Materials for Solid Hydrogen Storage: A Review. Int. J. Hydrog. Energy 2007, 32, 11211140

(231) Elsen, H.; Färber, C.; Ballmann, G.; Harder, S. LiAlH 4 : From Stoichiometric Reduction to Imine Hydrogenation Catalysis. Angew. Chem. Int. Ed. 2018, 57, 7156-7160. 
(232) Bismuto, A.; Cowley, M. J.; Thomas, S. P. Aluminum-Catalyzed Hydroboration of Alkenes. ACS Catal. 2018, 8, 2001-2005.

(233) Gieshoff, T. N.; Villa, M.; Welther, A.; Plois, M.; Chakraborty, U.; Wolf, R.; Jacobi von Wangelin, A. Iron-Catalyzed Olefin Hydrogenation at $1 \mathrm{Bar}_{2}$ with a FeCl $3-\mathrm{LiAlH}_{4}$ Catalyst. Green Chem. 2015, 17, 1408-1413.

(234) Less, R. J.; Simmonds, H. R.; Dane, S. B. J.; Wright, D. S. Stoichiometric and Catalytic Reactions of $\mathrm{LiAlH}_{4}$ with $\mathrm{Me}_{2} \mathrm{NHBH}_{3}$. Dalton Trans. 2013, 42, 63376343.

(235) Gardiner, M. G.; Lawrence, S. M.; Raston, C. L. Secondary Amine Stabilized Aluminum Hydrides Derived from $N, N^{\prime}$-Di-tert-butylethylenediamines. Inorg. Chem. 1996, 35, 1349-1354.

(236) Andrianarison, M. M.; Avent, A. G.; Ellerby, M. C.; Gorrell, I. B.; Hitchcock, P. B.; Smith, J. D.; Stanley, D. R. Complexes of Lithium Tetrahydroaluminate with $N, N, N^{\prime}, N^{\prime}$-tetramethylethane-1,2-diamine (tmen). Crystal Structures of $\left[\left\{\mathrm{Li}(\text { tmen })-\left(\mathrm{AlH}_{4}\right)\right\}_{2}\right]$ and $\left[\mathrm{Li}(\text { tmen })_{2}\right]\left[\mathrm{AlH}_{4}\right]$ and the Use of the ${ }^{6} \mathrm{Li}-\left\{{ }^{1} \mathrm{H}\right\}$ Nuclear Overhauser Effect to Study $\mathrm{LiAlH}_{4}$ and $\mathrm{LiBH}_{4}$ in Donor Solvents. J. Chem. Soc., Dalton Trans. 1998, 249-253.

(237) Gardiner, M. G.; Lawrence, S. M.; Raston, C. L. The Reaction of $\mathrm{LiAlH}_{4}$ with 1,4-Di-tert-butyl-1,4-diazabutadiene: Imine-Containing Aluminum Hydrides Stabilized by Lithium Coordination. Inorg. Chem. 1999, 38, 4467-4472.

(238) Kennedy, A. R.; McLellan, R.; McNeil, G. J.; Mulvey, R. E.; Robertson, S. D. Tetraamine $\mathrm{Me}_{6}$ TREN Induced Monomerization of Alkali Metal Borohydrides and Aluminohydrides. Polyhedron 2016, 103A, 94-99.

(239) Zheng, X.; Zhang, Z.; Tan, G.; Wang, X. An Aliphatic Solvent-Soluble Lithium Salt of the Perhalogenated Weakly Coordinating Anion $\left[\mathrm{Al}\left(\mathrm{OC}\left(\mathrm{CCl}_{3}\right)\left(\mathrm{CF}_{3}\right)_{2}\right)_{4}\right]^{\text {. }}$. Inorg. Chem. 2016, 55, 1008-1010.

(240) Lokare, K. S.; Wittwer, P.; Braun-Cula, B.; Frank, N.; Hoof, S.; Braun, T.; Limberg, C. Mimicking Base Interaction with Acidic Sites [Si-O(H)-Al] of Zeolites in Molecular Models. Z. Anorg. Allg. Chem. 2017, 643, 1581-1588.

(241) Less, R. J.; Allen, L. K.; Steiner, A.; Wright, D. S. Multiple Deprotonation of Primary Aromatic Diamines by $\mathrm{LiAlH}_{4}$. Dalton Trans. 2015, 44, 4141-4147. 
(242) Söhner, T.; Küppers, I. K.; Wackerow, W.; Rominger, F.; Straub, B. F. Synthesis and Structure of Salts of a Sterically Shielded, Lipophilic, $\mathrm{C}_{2}$-Symmetric, Fluxional Aluminate. ARKIVOC 2014, 15, 296-318.

(243) Söhner, T.; Braun, F.; Over, L. C.; Mehlhose, S.; Rominger, F.; Straub, B. F. Halogen-Free Water-Stable Aluminates as Replacement for Persistent Fluorinated Weakly-Coordinating Anions. Green Chem. 2014, 16, 4696-4707.

(244) Sambade, D.; Parkin, G. Synthesis and Structural Characterization of Tris(pyrazolyl)hydroaluminate and Tris(pyrazolyl)hydrogallate Lithium Compounds. Polyhedron 2017, 125, 219-229.

(245) Less, R. J.; Simmonds, H. R.; Wright, D. S. Reactivity and Catalytic Activity of tert-Butoxy-Aluminium Hydride Reagents. Dalton Trans. 2014, 43, 5785-5792.

(246) Pollard, V. A.; Orr, S. A.; McLellan, R.; Kennedy, A. R.; Hevia, E.; Mulvey, R. E. Lithium Diamidodihydridoaluminates: Bimetallic Cooperativity in Catalytic Hydroboration and Metallation Applications. Chem. Commun. 2018, 54, 1233-1236.

(247) Lemmerz, L. E.; McLellan, R.; Judge, N. R.; Kennedy, A. R.; Orr, S. A.; Uzelac, M.; Hevia, E.; Robertson, S. D.; Okuda, J.; Mulvey, R. E. Donor-influenced Structure-Activity Correlations in Stoichiometric and Catalytic Reactions of Lithium Monoamido-Monohydrido-Dialklyaluminates. Chem. Eur. J. 2018, 24, 9940-9948.

(248) Armstrong, D. R.; Crosbie, E.; Hevia, E.; Mulvey, R. E.; Ramsay, D. L.; Robertson, S. D. TMP (2,2,6,6-Tetramethylpiperidide)-Aluminate Bases: Lithium-Mediated Alumination or Lithiation-Alkylaluminium-Trapping Reagents? Chem. Sci. 2014, 5, 3031-3045.

(249) Conway, B.; Crosbie, E.; Kennedy, A. R.; Mulvey, R. E.; Robertson, S. D. Regioselective Heterohalogenation of 4-Halo-anisoles via a Series of Sequential ortho-Aluminations and Electrophilic Halogenations. Chem. Commun. 2012, 48, 4674-4676.

(250) Andrikopoulos, P. C.; Armstrong, D. R.; Clegg, W.; Gilfillan, C. J.; Hevia, E.; Kennedy, A. R.; Mulvey, R. E.; O’Hara, C. T.; Parkinson, J. A.; Tooke, D. M. A Homologous Series of Regioselectively Tetradeprotonated Group 8 Metallocenes: New Inverse Crown Ring Compounds Synthesized via a Mixed 
Sodium-Magnesium Tris(diisopropylamide) Synergic Base. J. Am. Chem.Soc. 2004, 126, 11612-11620.

(251) Naka, H.; Uchiyama, M.; Matsumoto, Y.; Wheatley, A. E. H.; McPartlin, M.; Morey, J. V.; Kondo, Y. An Aluminium Ate Base: Its Design, Structure, Function, and Reaction Mechanism. J. Am. Chem.Soc. 2007, 129, 1921-1930.

(252) Mulvey, R. E.; Armstrong, D. R.; Conway, B.; Crosbie, E.; Kennedy, A. R.; Robertson, S. D. Structurally Powered Synergic 2,2,6,6-Tetramethylpiperidine Bimetallics: New Reflections through Lithium-Mediated Ortho Aluminations. Inorg. Chem. 2011, 50, 12241-12251.

(253) Crosbie, E.; Garcia-Alvarez, P.; Kennedy, A. R.; Klett, J.; Mulvey, R. E.; Robertson, S. D. Structurally Engineered Deprotonation/Alumination of THF and THTP with Retention of Their Cycloanionic Structures. Angew. Chem. Int. Ed. 2010, 49, 9388-9391.

(254) Campbell, R.; Crosbie, E.; Kennedy, A. R.; Mulvey, R. E.; Naismith, R. A.; Robertson, S. D. Contrasting Reactivity of Mono- versus Bis-2,2,6,6tetramethylpiperidide Lithium Aluminates Towards Polydentate Lewis Bases: Co-Complexation Versus Deprotonation. Aust. J. Chem. 2013, 66, 1189-1201.

(255) Yang, Y.; Zhao, N.; Zhu, H.; Roesky, H. W. Syntheses and Reactions of Derivatives of (Pyrrolylaldiminato)germanium(II) and -Aluminum(III). Organometallics 2012, 31, 1958-1964.

(256) Nesterov, V.; Reiter, D.; Bag, P.; Frisch, P.; Holzner, R.; Porzelt, A.; Inoue, S. NHCs in Main Group Chemistry. Chem. Rev. 2018, 118, 9678-9842.

(257) Wang, Y.; Xie, Y.; Abraham, M. Y.; Wei, P.; Schaefer III, H. F.; Schleyer, P. v. R.; Robinson, G. H. A Viable Anionic N-Heterocyclic Dicarbene. J. Am. Chem.Soc. 2010, 132, 14370-14372.

(258) Hernan-Gomez, A.; Kennedy, A. R.; Hevia, E. C-N Bond Activation and Ring Opening of a Saturated N-Heterocyclic Carbene by Lateral Alkali-MetalMediated Metalation. Angew. Chem. Int. Ed. 2017, 56, 6632-6635.

(259) Fuentes, M. A.; Kennedy, A. R.; Mulvey, R. E.; Parkinson, J. A.; Rantanen, T.; Robertson, S. D.; Snieckus, V. Adding a Structural Context to the Deprotometalation and Trans-Metal Trapping Chemistry of PhenylSubstituted Benzotriazole. Chem. Eur. J. 2015, 21, 14812-14822. 
(260) Uzelac, M.; Kennedy, A. R.; Hevia, E.; Mulvey, R. E. Transforming LiTMP Lithiation of Challenging Diazines through Gallium Alkyl Trans-MetalTrapping. Angew. Chem. Int. Ed. 2016, 55, 13147-13150.

(261) McLellan, R.; Uzelac, M.; Kennedy, A. R.; Hevia, E.; Mulvey, R. E. LiTMP TransMetal-Trapping of Fluorinated Aromatic Molecules: A Comparitive Study of Aluminum and Gallium Carbanion Traps. Angew. Chem. Int. Ed. 2017, 56, 9566-9570.

(262) Appelt, C.; Slootweg, J. C.; Lammertsma, K.; Uhl, W. A Phosphorus/Aluminum-Based Frustrated Lewis Pair as an Ion Pair Receptor: Alkali Metal Hydride Adducts and Phase-Transfer Catalysis. Angew. Chem. Int. Ed. 2012, 51, 5911-5914.

(263) Dange, D.; Li, J.; Schenk, C.; Schnöckel, H.; Jones, C. Monomeric Group 13 Metal(I) Amides: Enforcing One-Coordination Through Extreme Ligand Steric Bulk. Inorg. Chem. 2012, 51, 13050-13059.

(264) Melton, C. E.; Dube, J. W.; Ragogna, P. J.; Fettinger, J. C.; Power, P. P. Synthesis and Characterization of Primary Aluminum Parent Amides and Phosphides. Organometallics 2014, 33, 329-337.

(265) Fettinger, J. C.; Gray, P. A.; Melton, C. E.; Power, P. P. Hydroalumination of Alkenes and Alkynes by Primary Aluminum Hydrides under Mild Conditions. Organometallics 2014, 33, 6232-6240.

(266) Garcia-Rodriguez, R.; Wright, D. S. Steric Effects on the Structures, Reactivity, and Coordination Chemistry of Tris(2-pyridyl)aluminates. Chem. Eur. J. 2015, $21,14949-14957$.

(267) Garcia-Rodriguez, R.; Hanf, S.; Bond, A. D.; Wright, D. S. A Non-Chiral Lithium Aluminate Reagent for the Determination of Enantiomeric Excess of Chiral Alcohols. Chem. Commun. 2017, 53, 1225-1228.

(268) Garcia-Rodriguez, R.; Bullock, T. H.; McPartlin, M.; Wright, D. S. Synthesis and Structures of Tris(2-pyridyl)aluminate Sandwich Compounds [ $\left.\left\{\mathrm{RAI}\left(2-\mathrm{py}{ }^{\prime}\right)_{2}\right\}_{2} \mathrm{M}\right]$ (py' = 2-pyridyl, M = Ca, Mn, Fe). Dalton Trans. 2014, 43, 14045-14053.

(269) Fernandez-Millan, M.; Temprado, M.; Cano, J.; Cuenca, T.; Mosquera, M. E. G. Synthesis of Novel Chiral Heterometallic Terpene Oximates: Unusual 
Generation of an Aluminium Enolate by a Cooperative Effect. Dalton Trans. 2016, 45, 10514-10518.

(270) Zhao, Y.; Lei, Y.; Dong, Q.; Wu, B.; Yang, X.-J. Reactivity of Dialumane and "Dialumene" Compounds toward Alkenes. Chem. Eur. J. 2013, 19, 1205912066.

(271) Segawa, Y.; Yamashita, M.; Nozaki, K. Boryllithium: Isolation, Characterization, and Reactivity as a Boryl Anion. Science 2006, 314, 113-115.

(272) Dettenrieder, N.; Schädle, C.; Maichle-Mössmer, C.; Anwander, R. Reactivity of Boryllithium with $\mathrm{AlMe}_{3}, \mathrm{AlEt}_{3}$, and $\mathrm{GaMe}_{3}$, Including the Synthesis of a Lanthanum Heterogallate Complex. Dalton Trans. 2014, 43, 15760-15770.

(273) Hicks, J.; Vasko, P.; Goicoechea, J. M.; Aldridge, S. Synthesis, Structure and Reaction Chemistry of a Nucleophilic Aluminyl Anion. Nature 2018, 557, 9295. 